\title{
BIOMASS-DERIVED HYDROGEN FROM A \\ THERMALLY BALLASTED GASIFIER
}

DOE Award Number:

DE-FC36-01GO11091

Final Technical Report

Reporting Period:

October 1, 2001 - September 31, 2006

\author{
Submitted By: \\ Iowa State University \\ 2207 Pearson Hall, Room 15 \\ Ames, IA 50011-2207
}

Principal Author(s):

Robert C. Brown, Jerod Smeenk, Samy Sadaka, Glenn Norton, Ruiqin Zhang, Andy Suby, Keith Cummer, Joe Ritzert, Ming Xu, Steve Lysenko, Josh Nunez, and Nathan Brown

Center for Sustainable Environmental Technologies

280 Metals Development Bldg.

Ames, IA 50011-3020

515-294-6555 / 515-294-3091 (fax)

rcbrown@iastate.edu 


\section{ABSTRACT \\ Biomass-Derived Hydrogen from a Thermally Ballasted Gasifier}

Principal Authors:

Robert C. Brown, Jerod Smeenk, Samy Sadaka, Glenn Norton, Ruiqin Zhang, Andy Suby, Keith Cummer, Joe Ritzert, Ming Xu, Steve Lysenko, Josh Nunez, and Nathan Brown

Center for Sustainable Environmental Technologies

280 Metals Development Bldg.

Ames, IA 50011-3020

515-294-6555 / 515-294-3091 (fax)

rcbrown@iastate.edu

The goal of this project is to develop an indirectly heated gasification system that converts switchgrass into hydrogen-rich gas suitable for powering fuel cells. The project includes investigations of the indirectly-heated gasifier, development of particulate removal equipment, evaluation of catalytic methods for upgrading producer gas, development of contaminant measurement and control techniques, modeling of the thermal performance of the ballasted gasifier, and estimation of the cost of hydrogen from the proposed gasification system. Specific technologies investigated include a thermally ballasted gasifier, a moving bed granular filter, and catalytic reactors for steam reforming and water-gas shift reaction.

The approach to this project was to employ a pilot-scale ( 5 ton per day) gasifier to evaluate the thermally ballasted gasifier as a means for producing hydrogen from switchgrass. A slipstream from the gasifier was used to evaluate gas cleaning and upgrading options. Other tests were conducted with laboratory-scale equipment using simulated producer gas.

The ballasted gasifier operated in conjunction with a steam reformer and two-stage water-gas shift reactor produced gas streams containing $54.5 \mathrm{vol}-\% \mathrm{H}_{2}$. If purge gas to the feeder system could be substantially eliminated, hydrogen concentration would reach 61 vol- $\%$, which closely approaches the theoretical maximum of $66 \mathrm{vol}-\%$. Tests with a combined catalyst/sorbent system demonstrated that steam reforming and water-gas shift reaction could be substantially performed in a single reactor and achieve hydrogen concentrations exceeding 90 vol- $\%$. Cold flow trials with a laboratory-scale moving bed granular filter achieved particle removal efficiencies exceeding $99 \%$. Two metal-based sorbents were tested for their ability to remove $\mathrm{H}_{2} \mathrm{~S}$ from biomass-derived producer gas. The $\mathrm{ZnO}$ sorbent, tested at $450^{\circ} \mathrm{C}$, was effective in reducing $\mathrm{H}_{2} \mathrm{~S}$ from $200 \mathrm{ppm}$ to less than $2 \mathrm{ppm}$ ( $>99 \%$ reduction) while tests with the $\mathrm{MnO}$ sorbent were inconclusive. A computer model was developed that successfully predicted the thermal performance of the ballasted gasifier. An economic comparison of an air-blown gasification plant and a ballasted gasifier plant found that operating costs for ballasted gasification plant are about $31 \%$ higher than for the air blown gasifier plant. Hydrogen from the ballasted gasification plant and air blown gasification plant are projected to be $\$ 2.43 / \mathrm{kg}$ and $\$ 1.85 / \mathrm{kg}$, respectively. This is lower than U.S. DOE's 2010 target price of $\$ 2.90 / \mathrm{kg}$ and comparable to U.S. DOE's 2015 target price of $\$ 2.00 / \mathrm{kg}$. 


\section{Table of Contents}

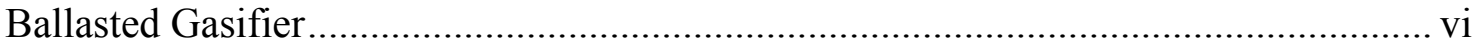

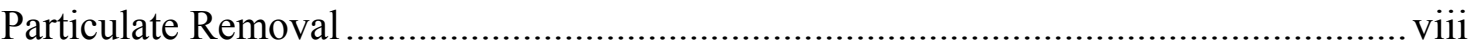

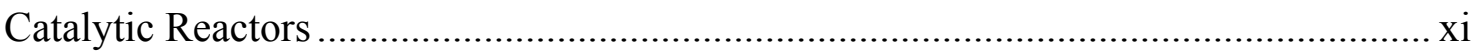

Contaminant Measurement and Control …............................................................... xii

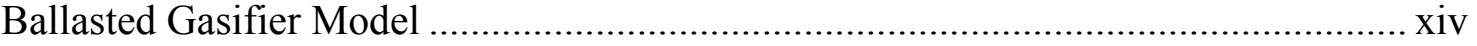

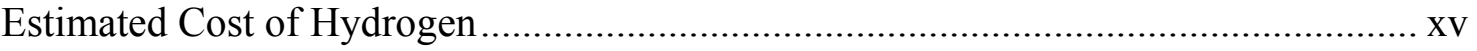

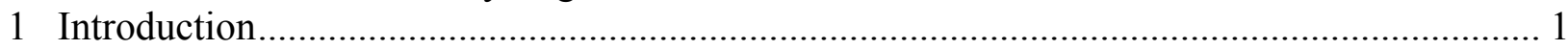

Description of Ballasted Gasifier ....................................................................... 1

Description of Moving Bed Granular Filter............................................................ 2

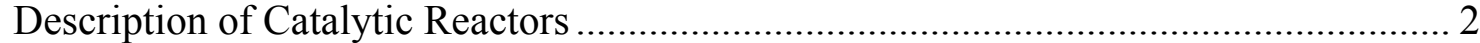

2 Experimental Apparatus and Methods............................................................................. 7

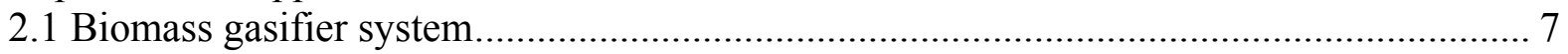

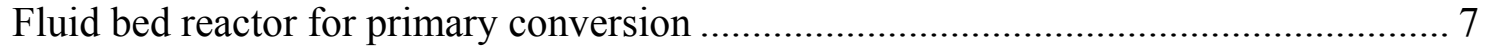

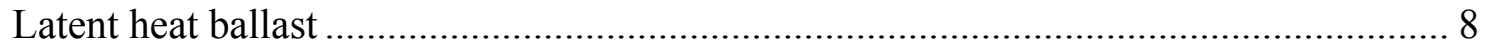

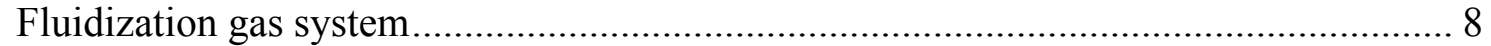

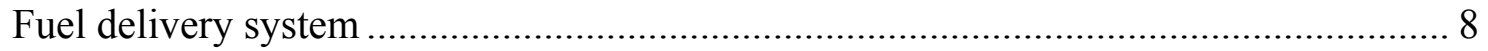

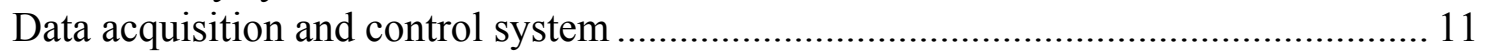

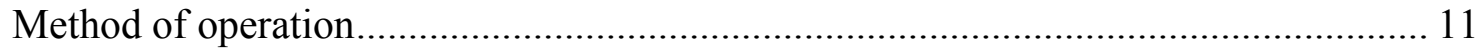

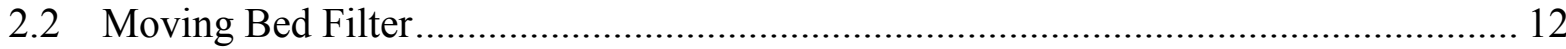

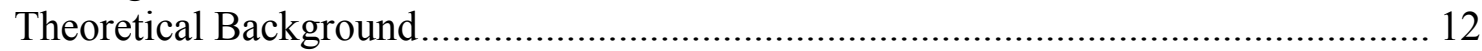

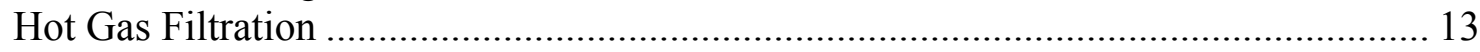

Cold Flow Experiments .............................................................................. 14

2.3 Catalytic Upgrading System ............................................................................ 15

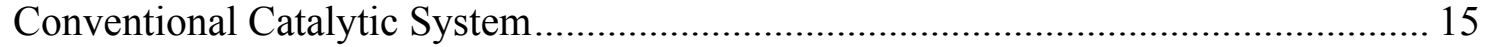

Combined Catalyst/Sorbent System .................................................................. 26

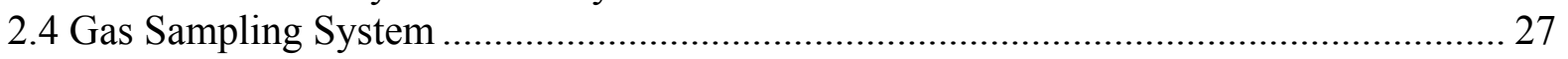

Trace Contaminant System ............................................................................. 27

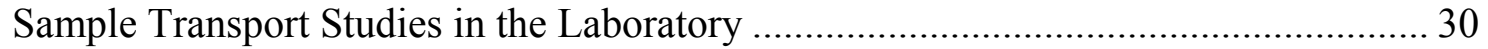

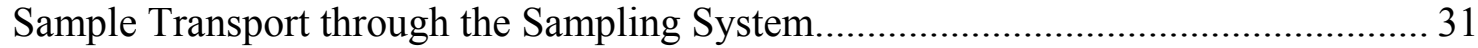

2.5 Gas and Trace Contaminant Analysis........................................................................ 32

Analysis of Major Gas Constituents .................................................................... 32

Sulfur Measurements ................................................................................ 32

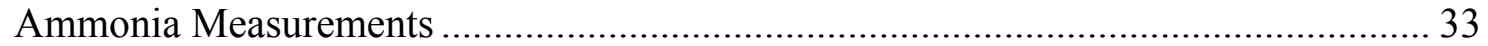

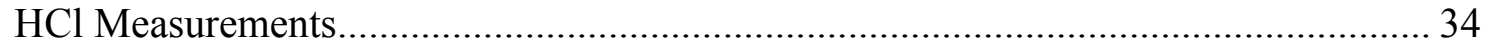

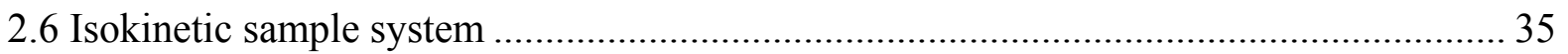

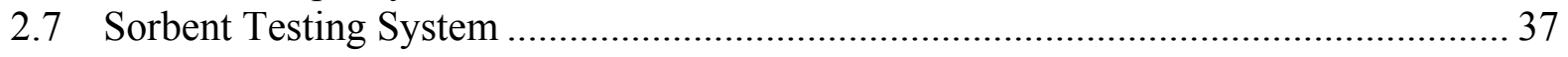

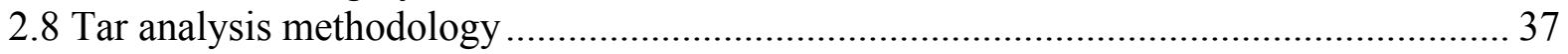

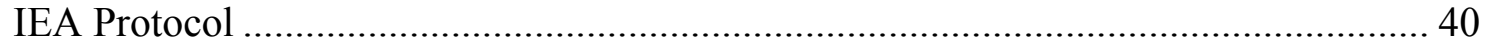

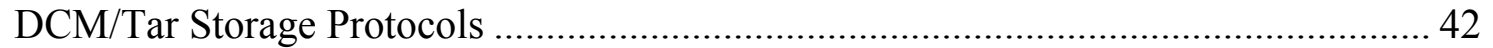

Hot Condenser Protocol ....................................................................................... 44

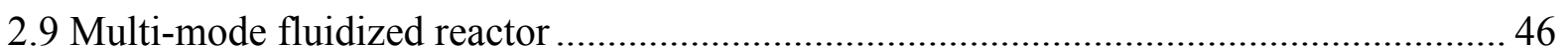

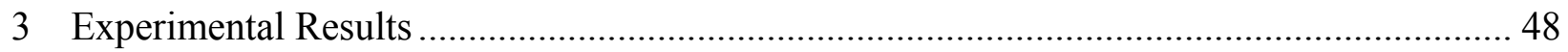

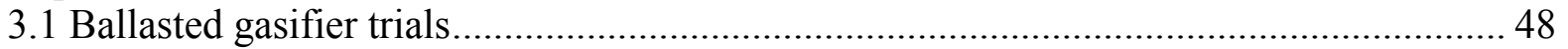

Reactor cooling time and cooling temperature profiles ..................................... 48 


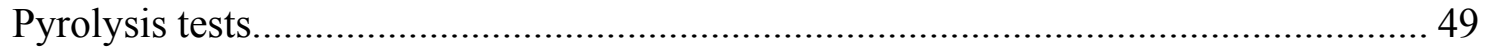

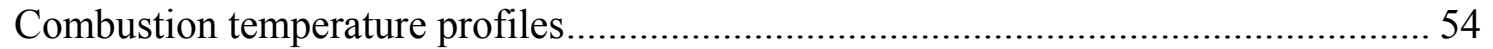

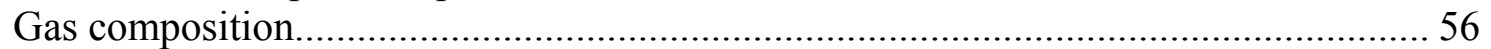

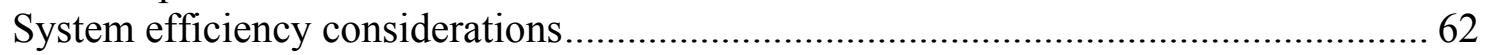

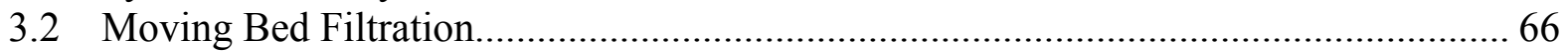

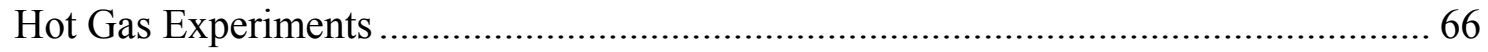

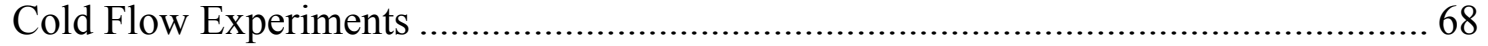

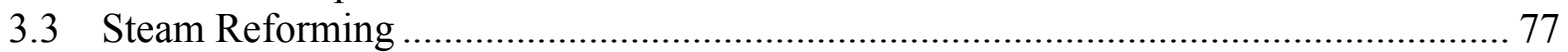

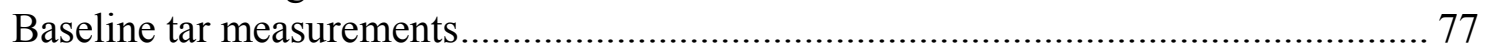

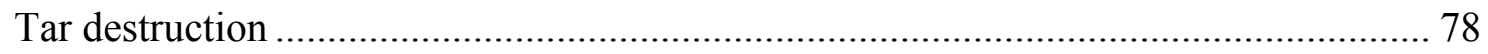

Effect of Catalytic Reactor Operating Conditions on Gas Composition ........................ 78

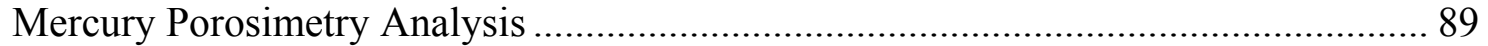

Carbon and sulfur analysis of catalysts and dolomite.................................................. 89

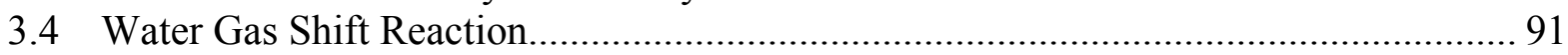

Conventional Water-Gas Shift Catalysts .................................................................... 91

Combined Catalyst/Sorbent System ……………….......................................... 105

3.5 Measurements of Ammonia and Sulfur .................................................................. 108

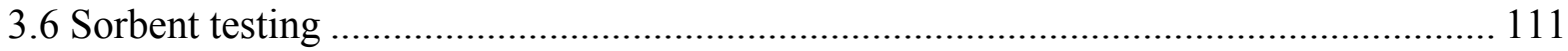

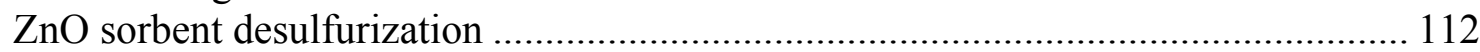

MnO-based sorbent desulfurization ...................................................................... 113

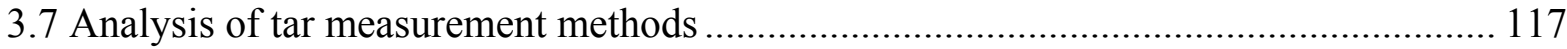

The Effect of Sample Aging on the Accuracy of the IEA Protocol............................. 117

Comparison Between the IEA Protocol and the Hot Condenser Methodology........... 118

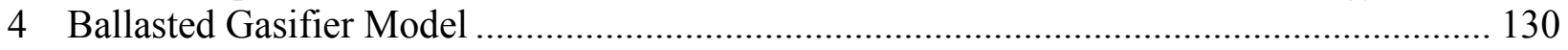

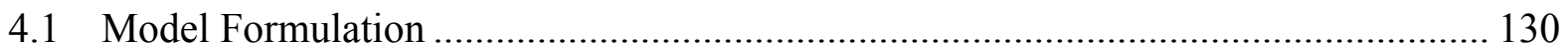

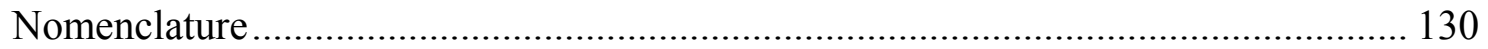

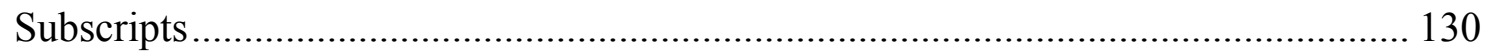

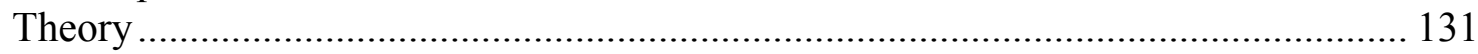

Model Assumptions ................................................................................................ 132

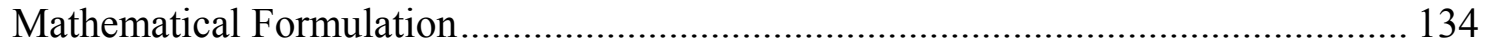

4.2 Computational Results ...................................................................................... 139

Sensitivity Analysis 1: Mass of phase-change material per tube................................ 139

Sensitivity Analysis 2: Thermal conductivity of lithium fluoride ............................. 142

Sensitivity Analysis 3: Characteristic dimension of the ballast................................. 143

5 Cost Estimating for Biomass Gasification Systems ………............................................... 148

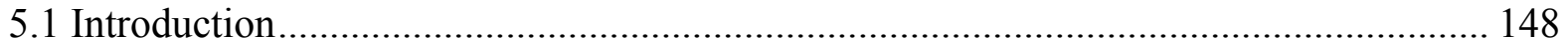

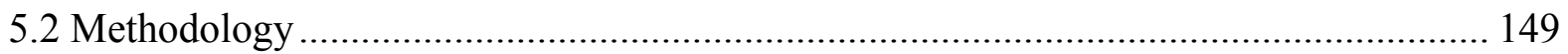

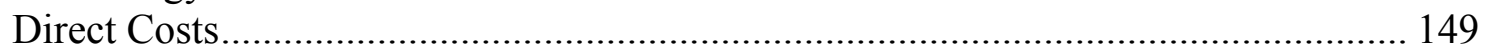

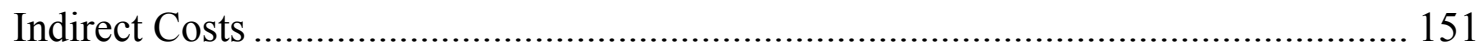

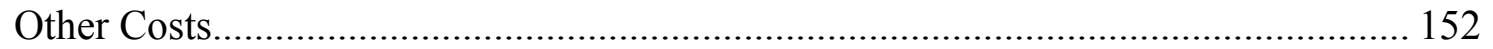

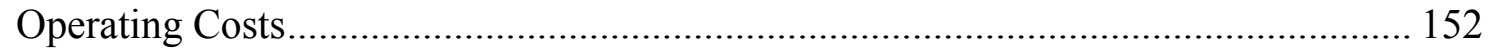

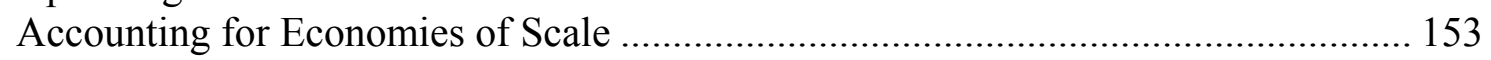

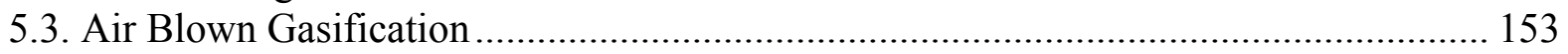

Mass Balance for Air Blown Gasification .................................................................. 153

Cost Estimate for Hydrogen from Air Blown Gasification ......................................... 154

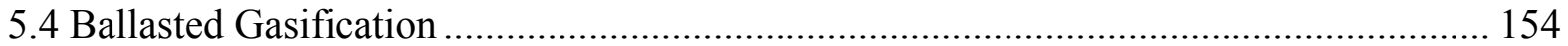


Mass Balance for Ballasted Gasification ..................................................................... 158

Energy Balance for Ballasted Gasification.................................................................. 159

Cost Estimate for Hydrogen from Ballasted Gasification ......................................... 159

Cost Estimate for 50 ton/day Hydrogen Production .................................................... 166

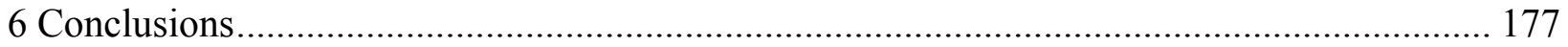

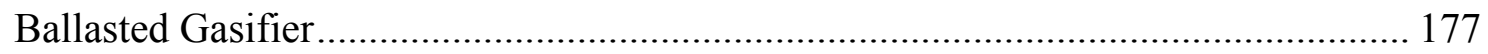

Moving Bed Granular Filter.................................................................................. 178

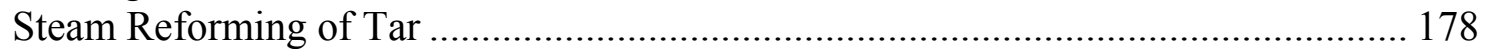

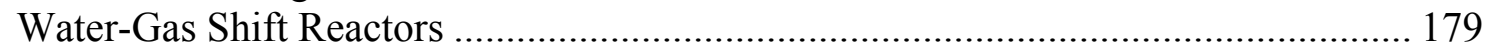

Measurements of Ammonia and Sulfur .................................................................. 180

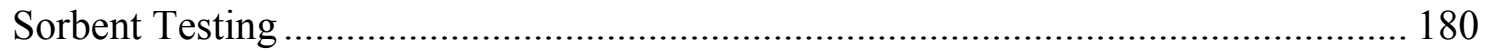

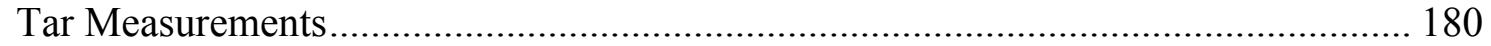

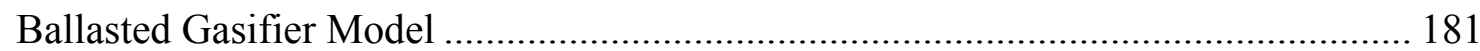

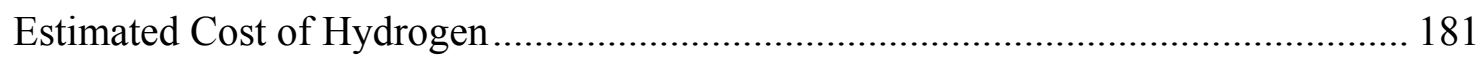

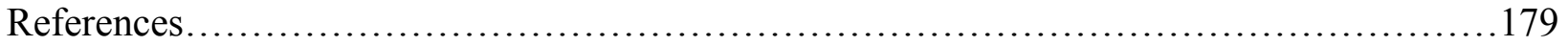




\section{Executive Summary}

The goal of this project is to develop an indirectly heated gasification system that converts switchgrass into hydrogen-rich gas suitable for powering fuel cells. The project includes investigations of the indirectly-heated gasifier, development of particulate removal equipment, evaluation of catalytic methods for upgrading producer gas, development of contaminant measurement and control techniques, modeling of the thermal performance of the ballasted gasifier, and estimation of the cost of hydrogen from the proposed gasification system. Specific technologies investigated include a thermally ballasted gasifier, a moving bed granular filter, and catalytic reactors for steam reforming and water-gas shift reaction.

The approach to this project was to employ a pilot-scale ( 5 ton per day) gasifier to evaluate the thermally ballasted gasifier as a means for producing hydrogen from switchgrass. Gasification at the pilot scale was important for obtaining realistic process data, especially for calculating energy flows through the system and assessing the practicality of feeding switchgrass into the gasifier.

A slipstream from the gasifier was used to evaluate gas cleaning and upgrading options. This slip stream included particulate filtration equipment; a guard bed designed to remove hydrogen sulfide and hydrogen chloride and some tar; a steam reformer designed to crack the remaining tar and decompose ammonia; and high temperature and low temperature catalytic water-gas shift reactors to remove carbon monoxide from the product gas and increase its hydrogen content.

Other tests were conducted with laboratory-scale equipment using simulated producer gas. These tests included evaluations of a combined steam reformer/water gas shift reactor using catalyst/sorbent pellets and cold-flow trials of the moving bed granular filter.

\section{Ballasted Gasifier}

Gasification usually consists of two distinct processes: combustion and pyrolysis. Pyrolysis is the chemical decomposition of solid fuel at elevated temperatures in the absence of oxygen to produce a combustible gas mixture. Since pyrolysis is an endothermic reaction, it must be accompanied by a source of heat. Conventional gasification admits sufficient air or oxygen to the reactor to burn part of the fuel, thus releasing heat to support pyrolysis of the rest of the fuel. Gas produced in air-blown biomass gasifiers typically has heating value only 10 to $20 \%$ that of natural gas and has hydrogen content of only $6-8 \%$.

An alternative approach, known as indirectly heated gasification, physically separates combustion and pyrolysis. As a result, the products of combustion are largely absent in the fuel gas. We have developed an indirectly heated gasifier that uses a single reactor for both combustion and pyrolysis. Instead of spatially separating these processes, they are temporally isolated; thus, the producer gas is not diluted with nitrogen. As illustrated in Fig. 1, the heat released during combustion at $850^{\circ} \mathrm{C}$ is stored as latent heat in the form of molten salt sealed in tubes immersed in the fluidized bed. During the pyrolysis phase, which occurs at temperatures between 600 and $850^{\circ} \mathrm{C}$, the reactor is fluidized with steam rather than air. Heat stored in the phase change material is released during this phase of the cycle to support the endothermic 
reactions of the pyrolysis stage. The reactor is designed as a fluidized bed to obtain uniform and rapid transfer of heat to and from the ballasting system immersed in the reactor.

The ballasted gasifier operates in a cycle, with a combustion phase followed by a pyrolysis phase. A larger amount of biomass can be processed through the reactor during the pyrolysis phase than during the combustion phase because essentially only very fast devolatilization of biomass occurs during the pyrolysis phase. Typically, the fuel flow rate during combustion was $55 \mathrm{~kg} / \mathrm{hr}$ compared to $180 \mathrm{~kg} / \mathrm{hr}$. These fuel flow rates produced a heating rate during combustion of about $19{ }^{\circ} \mathrm{C} / \mathrm{min}$ and a cooling rate during pyrolysis of $36^{\circ} \mathrm{C} / \mathrm{min}$. The average durations of the combustion and pyrolysis phases were 13.0 and 7.0 minutes, respectively. Thus, $11.9 \mathrm{~kg}$ of biomass is required to heat the reactor to pyrolyze $21 \mathrm{~kg}$ of biomass.

From an energy perspective, it is advantageous to process very high flow rates of biomass through the reactor during pyrolysis as possible since it is competing with the fixed steam flow rate through the reactor for heat stored in the ballast and sand bed. However, this results in a mismatch between the combustion (heating) period and the pyrolysis (cooling) phases, which averaged 13.0 minutes and 7.0 minutes, respectively. Thus, producer gas is only being generated during $35 \%$ of the cycle. Simultaneous operation of three reactors would be required to give continuous production of producer gas. Alternatively, increasing the heating rate during the combustion phase could yield a better match between the times to complete the two phases of the reactor. This could be achieved by reducing heat loss from the reactor and reducing the amount of purge nitrogen admitted to the reactor via the fuel feed system.

The performance of ballasted gasifier was evaluated both in terms of the hydrogen content of the raw producer gas and the hydrogen content after steam reforming and water-gas shift reaction. Raw producer gas from the ballasted gasifier contained 18 vol- $\% \mathrm{H}_{2}$ compared to 8 vol- $\% \mathrm{H}_{2}$ from the gasifier operated in air-blown mode. This value understates the potential of the ballasted gasifier to enhance hydrogen concentration because a substantial amount of nitrogen used to purge the fuel system entered the gasifier and diluted the producer gas during ballasted gasifier operation. Attempts to use smaller amounts of purge nitrogen were stymied by a tendency for the fuel feeder lines to overheat, which prevented proper feeding of fuel. However, if nitrogen purge could be substantially reduced through the use of an alternative fuel feed system, then hydrogen concentrations as high as 24 vol- $\%$ in the raw producer gas could be expected.

The raw producer gas contains substantial quantities of tar and carbon monoxide, which can be steam reformed and water-gas shifted, respectively, to additional hydrogen. When these systems were operated in conjunction with the ballasted gasifier, 54.5 vol- $\% \mathrm{H}_{2}$ was obtained with most of the balance being $\mathrm{CO}_{2}$ and nitrogen, the latter from the fuel feeder purge gas. If the purge gas could be substantially eliminated, hydrogen concentration would reach 61 vol- $\%$, which closely approaches the theoretical maximum of 66 vol- $\%$. By comparison, a conventional, air-blown gasifier produced hydrogen at concentrations less than 30 vol-\% on a dry, inert-free basis - less than half that of the ballasted gasifier. 


\section{Particulate Removal}

Moving bed granular filters were investigated as a means of removing particulate matter from producer gas streams. These filters operate on the principle that a flowing bed of particles can effectively scrub particulate contaminant from a gas stream. Although very promising for achieving high filtration efficiencies, the relatively large footprint of the equipment and high throughputs of granular material as filter media are cited as drawbacks to moving bed granular filters.

We have developed a new design that helps ameliorate these problems. Based on observations by other researchers that formation of a dust cake is important to efficient dust collection for fixed bed granular filters, we have developed a counter-flow arrangement that is intended to establish a dust cake at the interface between the gas and granular bed. As shown in Fig. 2, granular material moving downward by gravity spills out of a centrally located dipleg to form an interfacial region where most of the gas cleaning is hypothesized to occur. The lower edge of the filter cake is dispersed by the downward flow of granular material while the upper interface is covered by a fresh layer of granular material cascading from the dipleg above the interface. In this fashion, it is hypothesized that the interface establishes a dust cake of quasi-steady thickness, which is controlled to give high collection efficiency and acceptable pressure drop.

Both hot gas and cold gas evaluations of the moving bed concept for particulate filtration were performed. The hot gas trials were performed on a pilot-scale filter integrated with the biomass gasifier. Problems with maintaining gas seals in the sampling lines at high temperatures and confirming the existence of isokinetic sampling conditions limited the conclusions that could be drawn from the hot gas trials. Analysis of pressure drop data showed evidence of the development of a dust cake, as hypothesized for this kind of filter. In some trials the filtration efficiency reached $97 \%$ although these results were not repeatable with any degree of reliability. For this reason, the major effort on this part of the project shifted to cold flow trials, which produced more reliable data.

The cold flow trials were performed in a laboratory-scale moving bed granular filter using suspended fly ash or char in an ambient temperature air stream. These trials found efficiencies for removing fly ash to be as high as $99 \%$. A simple particle removal model was able to predict the performance of the filter. The bimodally distributed coal fly ash tested in this study required separate layer efficiencies to be employed in the model for the two sizes of particles. Very fine particles and very coarse particles were the most efficiently removed by the filter while particles in the size range of $33-128 \mu \mathrm{m}$ appeared to limit overall filter efficiency to about $99 \%$ even for gas residence times in the filter on the order of $1 \mathrm{~s}$. However, these middle-sized particles should be able to be removed by a high efficiency cyclone upstream of the moving bed filter, which would remove fine particles with efficiencies well in excess of $99 \%$. These experiments also showed that in excess of $86 \%$ of the fly ash was removed in the interfacial region where dusty gas first contacts the downward flowing granules of the moving bed.

In tests with other particulate matter (limestone, cornstarch, and oak char) it was found that filtration efficiency of the moving bed granular filter decreased with decreasing particle density and increasing irregularity of particle shape. Although oak char, consisting mostly of 


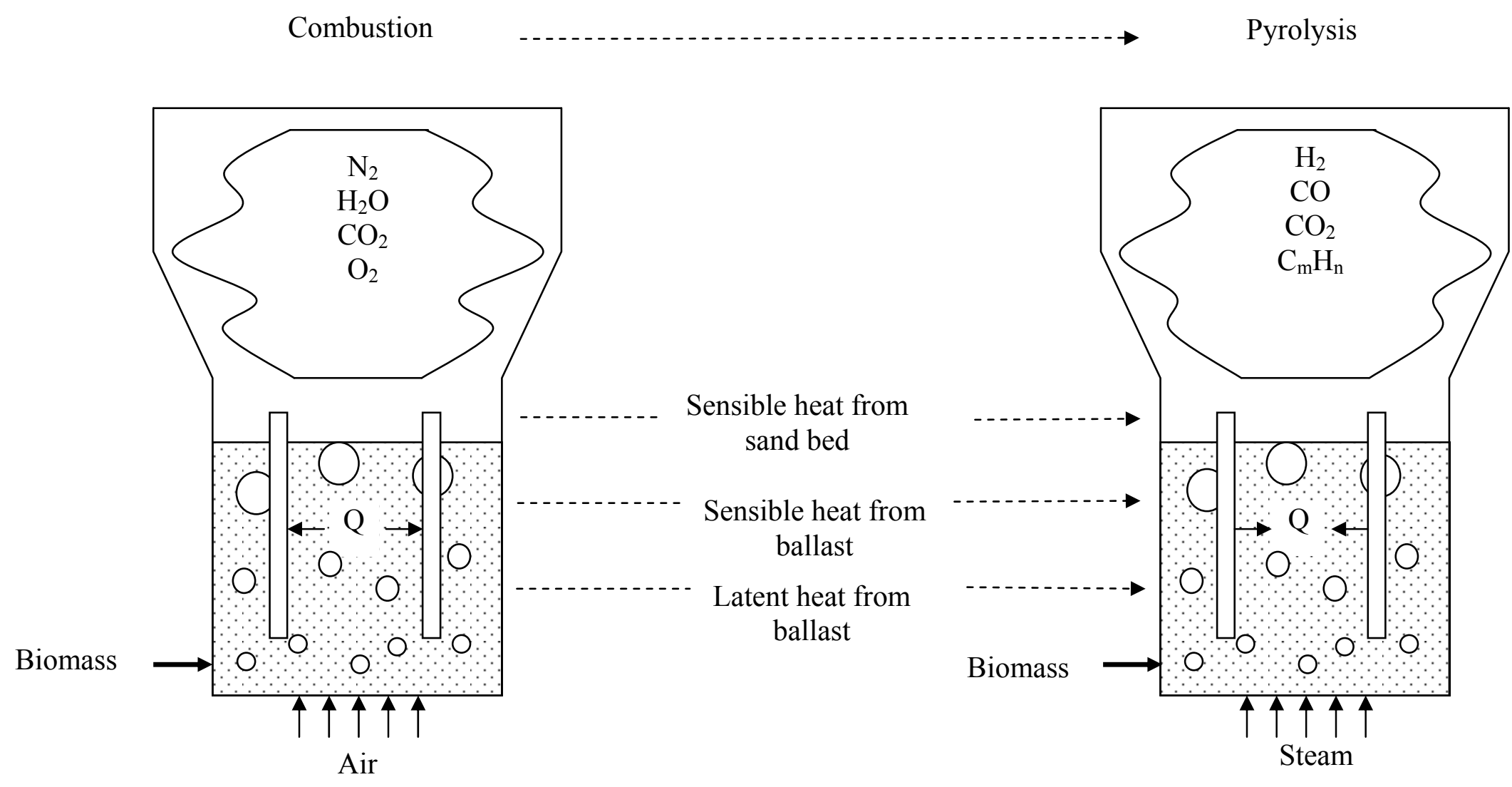

Fig. 1 Operating principle of the ballasted gasifier 


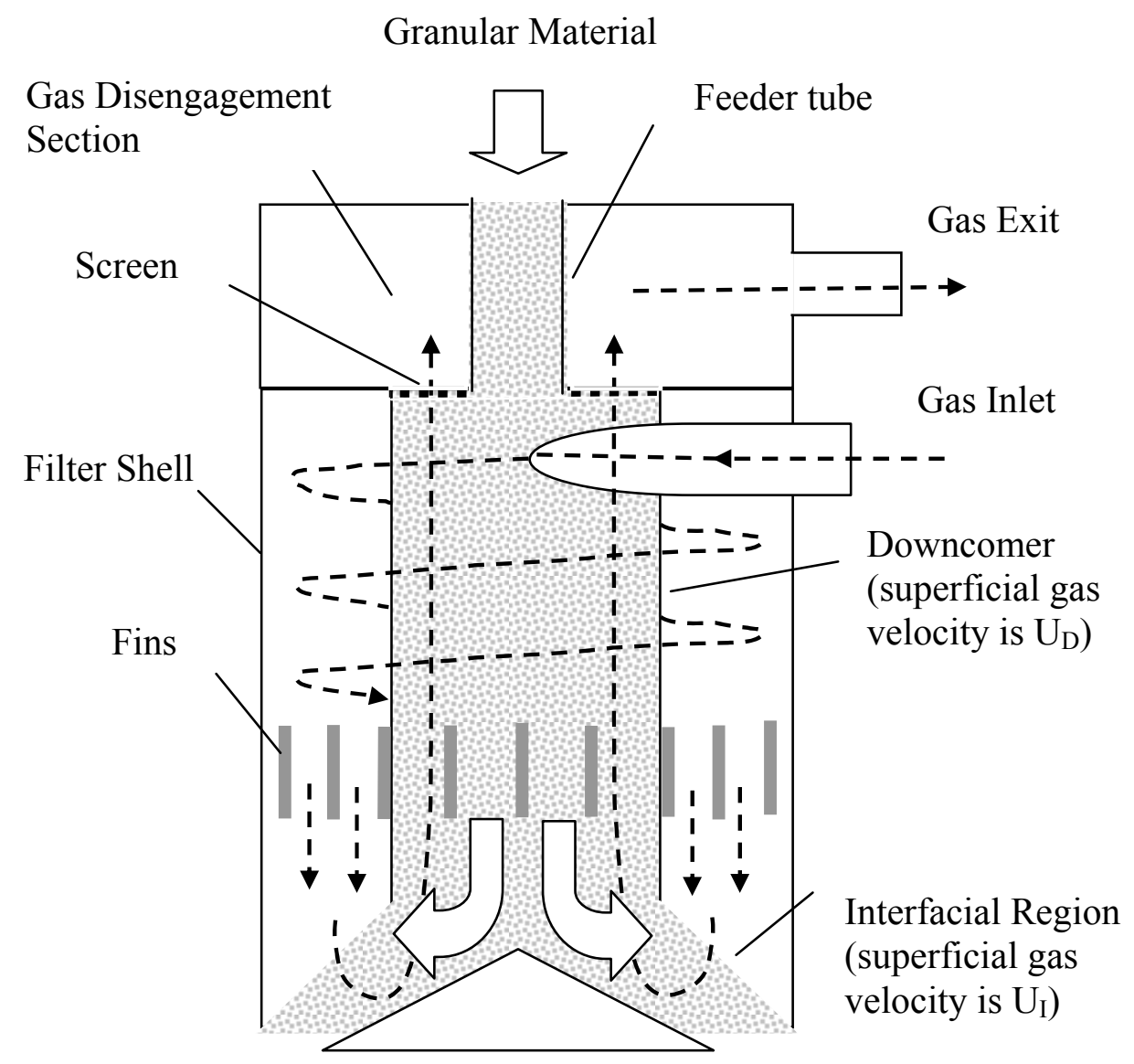

Fig. 2 Operating principle of the moving bed granular filter 
carbon, is expected to have very high van der Waals forces acting, the low density and highly irregular shape of this material resulted in collection efficiencies as low as $66 \%$. Since particle stickiness plays and important role in capture efficiency, it is hypothesized that higher efficiencies for char capture can be obtained by operating the filter at elevated temperatures, as would typically be employed in hot gas filtration. However, we were not able to perform such tests because the particle sampling methods were not suitable for elevated temperatures.

\section{Catalytic Reactors}

Catalytic reactors were investigated for their usefulness in steam reforming of tars in the producer gas and promoting the water-gas shift reaction for enhancing hydrogen yields. Some of these studies employed separate reactors for steam reforming and high and low temperature water-gas shift reaction. In the last year of the project, a combined catalyst/sorbent system was investigated, which has prospects for replacing three catalytic reactors with a single reactor.

Investigations of steam reforming of tar evaluated three metal catalysts (ICI 46-1, Z409, and RZ409). All three proved effective in eliminating heavy tars ( $>99 \%$ destruction efficiency) and in increasing hydrogen concentration by 6-11 vol-\% (dry basis). Space velocity had little effect on gas composition while increasing temperature boosted hydrogen yield and reduced light hydrocarbons ( $\mathrm{CH} 4$ and $\mathrm{C} 2 \mathrm{H} 4)$, thus suggesting tar destruction is controlled by chemical kinetics.

Although reactivity of the steam reforming system did not diminish during the $12-18$ hours of testing, measurement of surface area and pore size distribution indicated the conversion of small pores into larger pores during high temperature operation. If this transformation were to continue, catalytic activity would eventually degrade. Furthermore, coke accumulated on both the dolomite and metallic catalysts although this might have been mitigated if higher steam/TOC ratios had been employed from the beginning of the tests.

Steam reforming trials were followed by tests of a conventional two-stage water gas shift reactor system consisting of separate stages for high temperature shift reaction and low temperature shift reaction. This system, operated in conjunction with a steam reformer operated upstream of the shift reactors, was able to upgrade the hydrogen content of raw producer gas from the gasifier operated in air-blown mode from 8.6 vol- $\%$ to 26.7 vol- $\%$. Carbon monoxide concentration of 14.3 vol- $\%$ in the raw producer gas was reduced to less than 0.2 vol-\%. Carbon monoxide conversion in the high temperature shift reactor reached $83 \%$ while the overall conversion in the two-stage shift reaction system reached $98.7 \%$. Although the steam reformer destroyed essentially all condensable tars and significantly reduced $\mathrm{C}_{2} \mathrm{H}_{4}$, it reformed very little of the $\mathrm{CH}_{4}$, which may reflect thermodynamic limitations as a result of inadequate temperature and too high of partial pressure of $\mathrm{CO}_{2}$.

Characterization of the catalysts by x-ray photoelectron spectroscopy showed that coke and small quantities of sulfur and chlorine deposited on the catalysts. BET analysis revealed loses in micropores and mesopores. Although no sign of catalytic deactivation was evident during the tests, these changes indicate the need for improvements in the design of the guard bed. 
This part of the project then turned to the development of a system able to perform steam reforming and high temperature and low temperature water gas shift reaction in a single reactor. This was accomplished with pellets that combine catalytic reaction with carbon dioxide adsorption. These core-in-shell catalyst-sorbent pellets, illustrated in Fig. 3, consist of spherical pellets in which a $\mathrm{CaO}$ core (typically $3.4-4.0 \mathrm{~mm}$ in diameter) is surrounded by a porous but strong protective shell made of alumina (typically $0.3-0.5 \mathrm{~mm}$ in thickness) that supports a catalyst for both the steam reforming and the water gas shift reaction. During the hydrogen production period, the material serves two functions in catalyzing the reaction of hydrocarbons or carbon monoxide with steam to produce hydrogen while simultaneously absorbing carbon dioxide formed by the reaction. Efficient $\mathrm{CO}_{2}$ absorption is obtained at reaction temperature less than $600^{\circ} \mathrm{C}$ at $1.0 \mathrm{~atm}$ pressure. The in-situ removal of $\mathrm{CO}_{2}$ shifts the equilibrium of the steam reforming reaction and/or water gas shift reaction to increase the hydrogen concentration and simultaneously decreases the concentration of carbon oxides in the producer gas stream until the sorbent is saturated. The sorbent can be regenerated by simply heating the pellets (for instance by directly/indirectly passing flue gas) to $750^{\circ} \mathrm{C}$ to remove $\mathrm{CO}_{2}$.

Since the tests performed were exploratory in nature, they were performed on simulated producer gas rather than producer gas generated from the biomass gasifier. These tests revealed that in the absence of $\mathrm{CO}_{2}$ adsorption, the hydrogen concentration never exceeded 70 vol- $\%$ due to the high concentration of $\mathrm{CO}_{2}$ arising from the water-gas shift reaction. However, when $\mathrm{CO}_{2}$ absorption by the pellets was allowed to occur, hydrogen concentrations exceeded 90 vol-\%. Furthermore, it appears that the catalyst was not only effective in promoting the water-gas shift reaction but in reforming some of the methane in the producer gas.

Following the test with steam and toluene some carbon was found deposited on the surface of the pellets and on the wall of the reactor above the bed indicating thermal decomposition of the toluene. This problem may be solved by increasing the steam to carbon ratio or lowering the operating temperature.

\section{Contaminant Measurement and Control}

Detection of trace contaminants in producer gas streams using continuous, on-line instruments is challenging because of the presence of a wide variety of organic and inorganic compounds that can interfere with the desired measurements. Potentially attractive techniques like Fourier Transform Infrared Spectroscopy (FTIR), ion chromatography, and various continuous emission monitoring (CEM) instruments offered commercially proved inadequate for use with tar-laden producer gas. We eventually settled upon Drager tubes for repeatable although low resolution measurement of $\mathrm{H}_{2} \mathrm{~S}$ and wet chemical methods for accurate though tedious measurement of ammonia.

In some tests, the concentration of $\mathrm{NH}_{3}$ appeared to substantially decrease with time. This unexpected behavior is believed to be related to the accumulation of high ash loading in the thimble filter used upstream of the impingers to remove particulate matter. We suspect that the $\mathrm{NH}_{3}$ was interacting with the ash, a caution for others using similar methods for ammonia detection. 


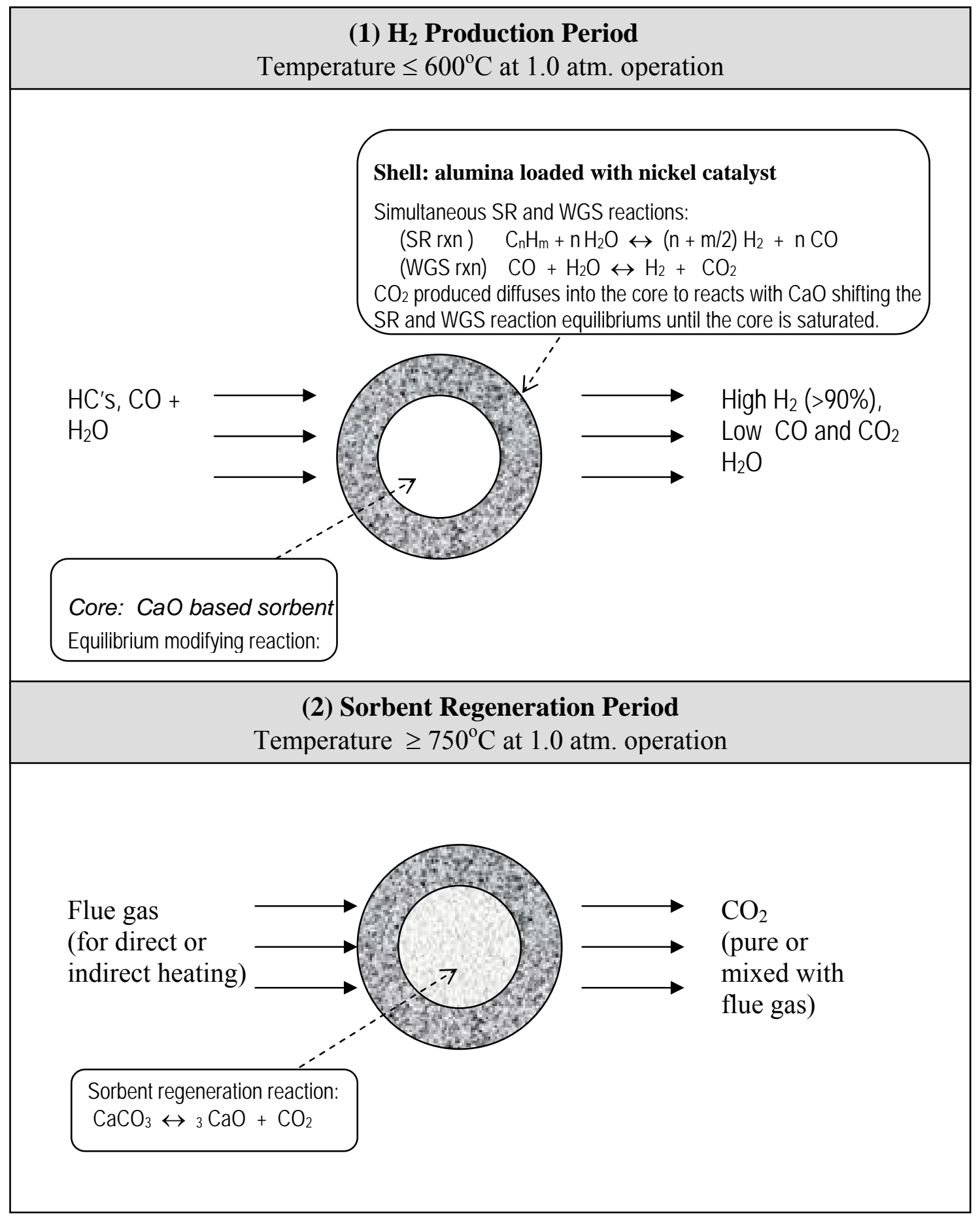

Fig. 3 Schematic of a core-in-shell catalyst-sorbent pellet and reaction steps involved in producing high purity $\mathrm{H}_{2}$ from hydrocarbons and/or CO. and in regenerating the sorbent. 
Two metal-based sorbents were tested for their ability to remove $\mathrm{H}_{2} \mathrm{~S}$ from biomass-derived producer gas. The $\mathrm{ZnO}$ sorbent, tested at $450^{\circ} \mathrm{C}$, was effective in reducing $\mathrm{H}_{2} \mathrm{~S}$ from $200 \mathrm{ppm}$ to less than $2 \mathrm{ppm}$ ( $>99 \%$ reduction). The MnO-based sorbent, tested at $375^{\circ} \mathrm{C}$, showed evidence that it could reduce $\mathrm{H}_{2} \mathrm{~S}$ from $200 \mathrm{ppm}$ to less than $20 \mathrm{ppm}$ ( $>90 \%$ reduction), but anomalous behavior in the course of the experiment suggested that channeling or some related behavior was occurring through the packed bed, preventing more definitive conclusions from being drawn from the test.

The Wheeler equation, originally developed for analyzing the performance of gas masks, was employed in analyzing some of the data. This equation offers a means for determining both sorption rates and capacities during tests that are much shorter in duration than required to get actual saturation of a filter. This could be a very powerful methodology for the development of sorbents for the gasification industry. However, this methodology requires the ability to collect time series data on contaminant concentrations exiting the sorbent bed. The Drager tubes proved inadequate for this purpose. Recent installation of a GC-SCD system for semi-continuous measurement of sulfur species is expected to make this kind of analysis possible in future testing.

Tar is another contaminant requiring accurate measurement. Both the evaporative method of the IEA Protocol for tar measurement and a technique developed at Iowa State University were evaluated for their usefulness in measuring tar concentrations in biomass producer gas. Aging trials on tar/DCM samples collected by the IEA protocol demonstrated that storage of these samples, for as little as six hours, will produce errors in the reported tar concentration. The apparent tar concentrations can increase or decrease with time, depending upon the storage protocol. Thus, analysis of tar/DCM samples is recommended immediately upon collection of a sample.

The alternative tar analysis method developed at ISU is designed to simplify the analytical method while yielding results that are comparable to the evaporative method of the IEA Protocol (that is, determination of the heavy tar content of producer gas). This method, premised on condensing heavy tar at temperatures slightly higher than the boiling point of water, consists of simple gravimetric methods applied to a disposable tube. This "hot condenser" method yielded highly repeatable results that were within $10 \%$ of the values obtained by the IEA Protocol (for which no independent determination of accuracy is available).

\section{$\underline{\text { Ballasted Gasifier Model }}$}

The purpose of this model was to accurately predict the thermal performance of the ballast system used in the biomass gasifier. A Receding Interface (RI) model was developed to account for the thermal barrier that develops within the ballast tubes as the LiF phase change material solidifies around the periphery of the tubes. This model predicts a continuous decrease in temperature during steam cooling of the fluidized bed, which is consistent with the behavior seen in experimental tests. The model demonstrates that the build-up of solid LiF during phase change slows heat transfer sufficiently to prevent a period of constant temperature reaction during the pyrolysis phase as was expected from earlier thermal models of the gasifier. The RI model predicts temperature profiles that are at slightly higher temperatures than are experimentally observed, but this is not unexpected since the model is one-dimensional.

Sensitivity analyses were performed on three parameters yielding the following conclusions: 
- The time required to cool the bed to a particular temperature is proportional to the amount of phase change material employed in the ballast.

- Thermal conductivity of the lithium fluoride affects the cooling curves, but only during the period of phase change. Once the phase change material solidifies, the cooling curves are essentially identical.

- For a given mass of phase change material, smaller diameter tubes more closely approach the isothermal cooling curves of the idealized phase change process than do larger tubes.

\section{Estimated Cost of Hydrogen}

This evaluation compared the cost of hydrogen from biomass using the proposed ballasted gasifier and a conventional air-blown gasifier. For a plant producing 50 tpd of hydrogen, the total capital cost for the ballasted gasifier system is $\$ 31.99$ million compared to $\$ 19.46$ million for an air-blown system. The addition of a steam generator and ballast system leads to higher capital cost for ballasted gasification.

Hydrogen from the air blown gasification plant is projected to be $\$ 1.85 / \mathrm{kg}$ whereas hydrogen from the ballasted gasification plant is slightly higher at $\$ 2.43 / \mathrm{kg}$. These projections are within the range projected by other biomass-to-hydrogen analyses (between $\$ 1.38$ and $\$ 6.10 / \mathrm{kg}$ ) and less than the 2010 target price of the U.S. DOE $(\$ 2.90 / \mathrm{kg})$. The major determinant of the cost of hydrogen is the cost of biomass used in the process, which was assumed to be $\$ 55 /$ ton for the present analysis. 


\section{Introduction}

The goal of this project is to develop an indirectly heated gasification system that converts switchgrass into hydrogen-rich gas suitable for powering fuel cells. The project includes investigations of the indirectly-heated gasifier, development of particulate removal equipment, evaluation of catalytic methods for upgrading producer gas, development of contaminant measurement and control techniques, modeling of the thermal performance of the ballasted gasifier, and estimation of the cost of hydrogen from the resulting gasification system. Specific technologies investigated include a thermally ballasted gasifier, a moving bed granular filter, and catalytic reactors for steam reforming and water-gas shift reaction.

\section{Description of Ballasted Gasifier}

Gasification usually consists of two distinct processes ${ }^{1}$ : combustion and pyrolysis. Pyrolysis is the chemical decomposition of solid fuel at elevated temperatures in the absence of oxygen to produce a combustible gas mixture. Since pyrolysis is an endothermic reaction, it must be accompanied by a source of heat. Conventional gasification admits sufficient air or oxygen to the reactor to burn part of the fuel, thus releasing heat to support pyrolysis of the rest of the fuel. Gas produced in air-blown biomass gasifiers typically has heating value only 10 to $20 \%$ that of natural gas and has hydrogen content of only $6-8 \%$.

An alternative approach, known as indirectly heated gasification, physically separates combustion and pyrolysis. As a result, the products of combustion are largely absent in the fuel gas. Higher heating values of $14,200 \mathrm{~kJ} / \mathrm{m}^{3}$ (380 BTU/scf) or higher are expected. Several schemes have been suggested for transporting heat from the combustion reactor to the pyrolysis reactor. These include transferring hot solids from the combustor to the pyrolyzer ${ }^{2}$, transferring a chemically regenerative heat carrier between two reactors ${ }^{3}$, and transferring heat through a wall common to the reactors ${ }^{4}$. These schemes are not well suited to the relatively small scales envisioned for rural heat and power generation.

We have developed an indirectly heated gasifier that uses a single reactor for both combustion and pyrolysis ${ }^{5}$. Instead of spatially separating these processes, they are temporally isolated; thus, the producer gas is not diluted with nitrogen. As illustrated in Figure 1, the heat released during combustion at $850^{\circ} \mathrm{C}$ is stored as latent heat in the form of molten salt sealed in tubes immersed in the fluidized bed. During the pyrolysis phase, which occurs at temperatures between 600 and $850^{\circ} \mathrm{C}$, the reactor is fluidized with steam rather than air. Heat stored in the phase change material is released during this phase of the cycle to support the endothermic reactions of the pyrolysis stage. The reactor is designed as a fluidized bed to obtain uniform and rapid transfer of heat to and from the ballasting system immersed in the reactor.

In practice, this latent heat ballasting system consists of an array of stainless steel tubes enclosing high temperature phase-change material. The array of tubes is immersed within the bed of a fluidized reactor, which assures high rates of heat transfer between tubes and biomass. The ideal phase change temperature would be close to the nominal combustion temperature of the fluidized reactor $\left(850^{\circ} \mathrm{C}\right)$ and have large heat of fusion on a volumetric basis. Of course, the material should be non-flammable and relatively inexpensive. A large number of high 
temperature phase-change materials were surveyed for this application. We have settled on lithium fluoride (LiF), a stable salt that does not react with stainless steel, as ballast material. Lithium fluoride has an ideal melting point $\left(848^{\circ} \mathrm{C}\right)$ and high latent heat of fusion $\left(1800 \mathrm{MJ} / \mathrm{m}^{3}\right)$.

Because air is not used during the gas-producing phase of the cycle, nitrogen does not dilute the product gas, resulting in relatively high concentrations of hydrogen and carbon monoxide in the producer gas compared to conventional gasifiers. In early trials we measured hydrogen content between 20 and 36 vol-\%, which is a factor of 4 to 6 higher than found in gas from air-blown gasifiers. The thermal ballasting system also more than doubles the carbon monoxide concentration. This carbon monoxide, along with steam used to fluidize the reactor, can be shifted to additional hydrogen by the water-gas shift reaction.

\section{Description of Moving Bed Granular Filter}

Moving bed granular filters operate on the principle that a flowing bed of particles can effectively scrub particulate contaminant from a gas stream. Although very promising for achieving high filtration efficiencies, the relatively large footprint of the equipment and high throughputs of granular material as filter media are cited as drawbacks to moving bed granular filters.

We have developed a new design that helps ameliorate these problems. Based on observations by other researchers that formation of a dust cake is important to efficient dust collection for fixed bed granular filters, we have developed a counter-flow arrangement that is intended to establish a dust cake at the interface between the gas and granular bed. As shown in Figure 2, granular material moving downward by gravity spills out of a centrally located dipleg to form an interfacial region where most of the gas cleaning is hypothesized to occur. The lower edge of the filter cake is dispersed by the downward flow of granular material while the upper interface is covered by a fresh layer of granular material cascading from the dipleg above the interface. In this fashion, it is hypothesized that the interface establishes a dust cake of quasi-steady thickness, which is controlled to give high collection efficiency and acceptable pressure drop. Further details on its operating principles are found in Brown et al. ${ }^{6}$

\section{Description of Catalytic Reactors}

Methods to remove tars from producer gas can generally be classified into one of three categories: physical processes, thermal processes or catalytic processes. Physical processes, such as filters or wet scrubbers, remove the tar from the producer gas through gas/solid or gas/liquid interactions. While these methods are effective and relatively easy to maintain, they do not truly alleviate the problem, as the tar is not destroyed and environmentally responsible disposal of the resulting tar-laden filter material is difficult. Thermal processes raise the temperature of the producer gas to levels that "crack" heavy aromatic tar species into lighter and less problematic species, such as hydrogen, carbon monoxide and methane. For thermal cracking of tars, temperatures exceeding $1000^{\circ} \mathrm{C}$ are required to effectively reduce tars, which may be difficult to achieve in biomass thermochemical systems ${ }^{7}$.

Catalytic processes can operate at much lower temperatures $\left(600-800^{\circ} \mathrm{C}\right)$ than thermal process, alleviating the need for expensive alloys for reactor construction. Depending on the catalytic process, this temperature range may eliminate the need to heat and/or cool the producer gas as it 


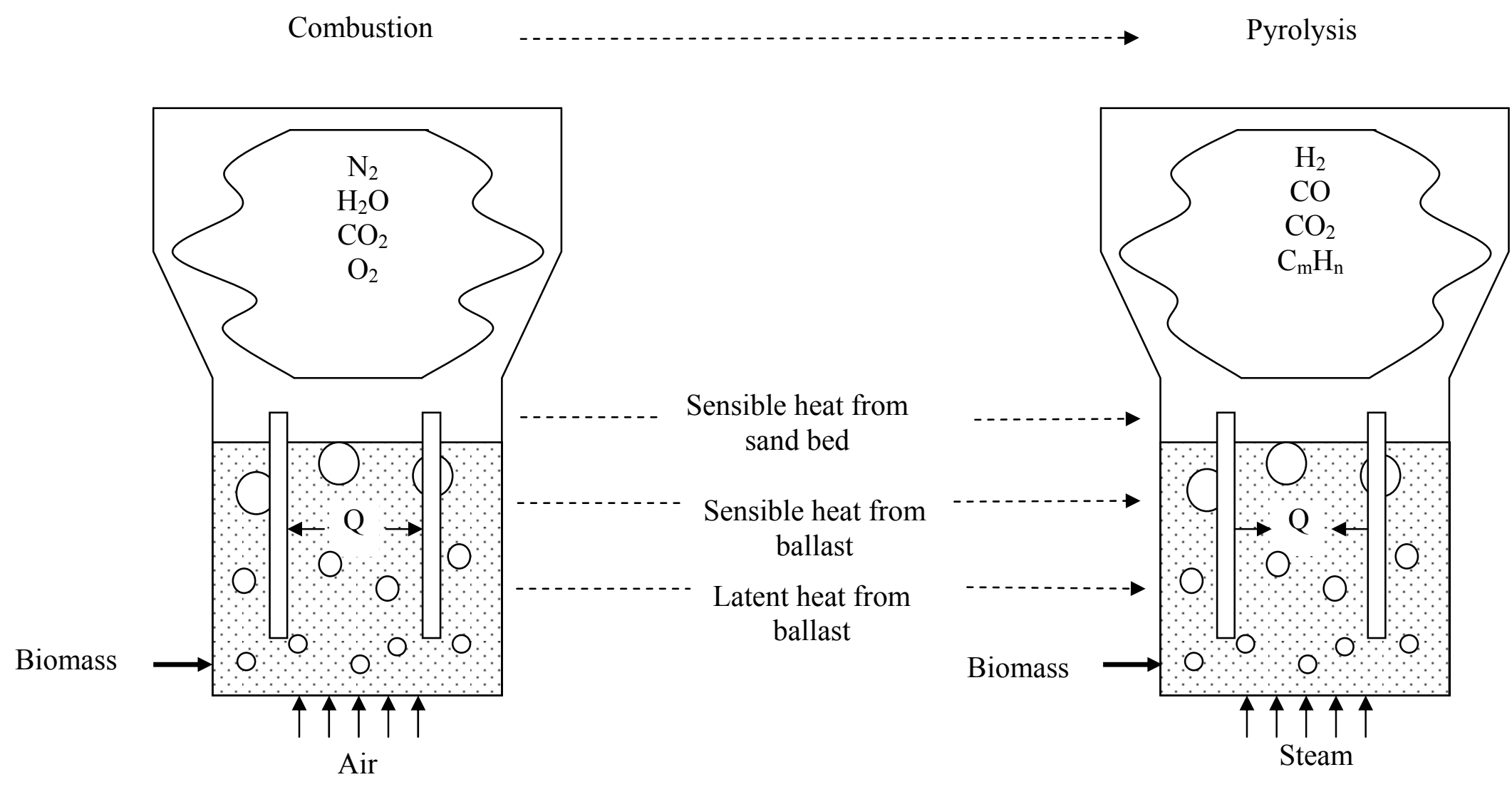

Figure 1 Operating principle of the ballasted gasifier 


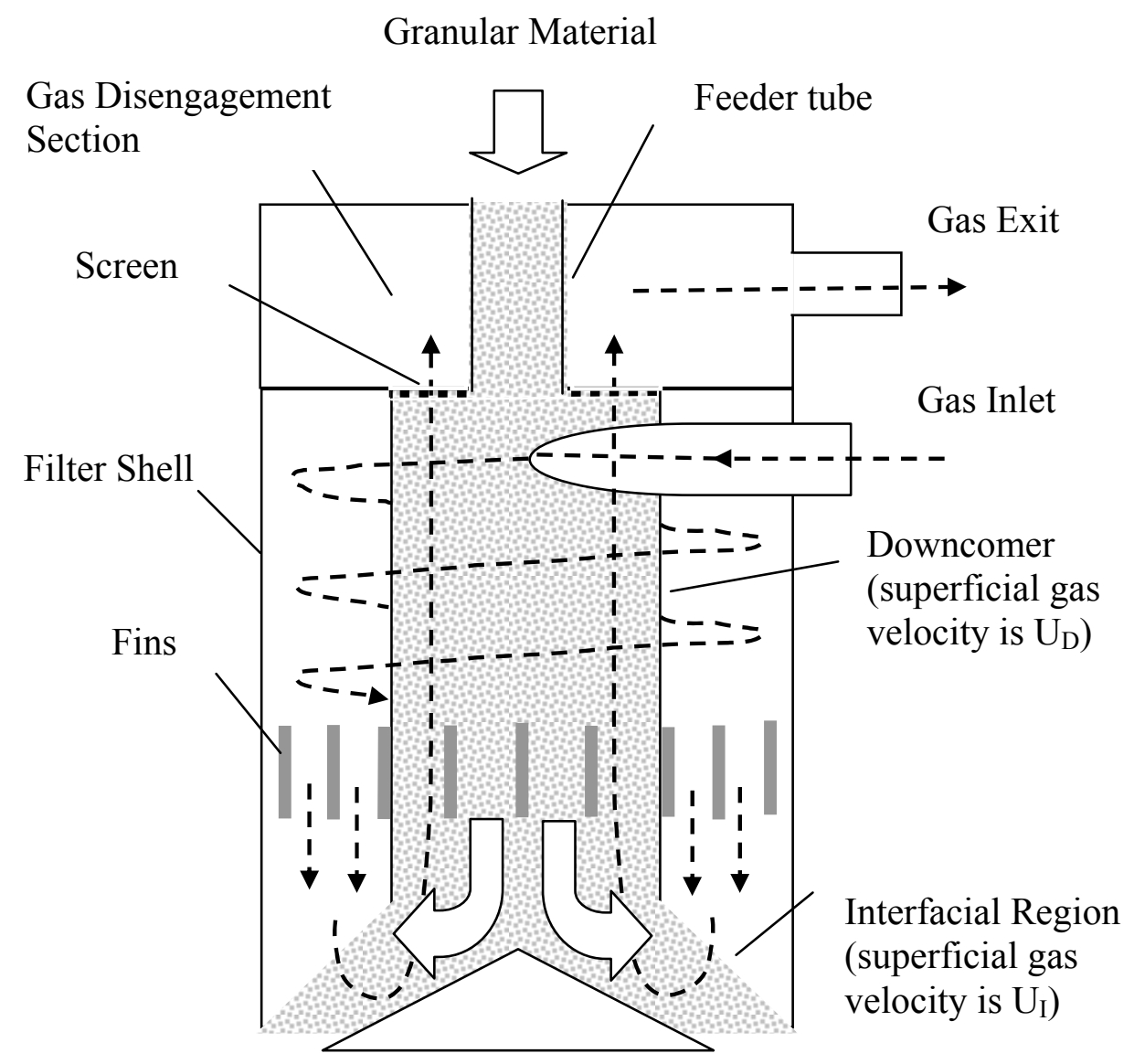

Figure 2 Operating principle of the moving bed granular filter 
leaves the gasifier. Also, unlike physical processes, catalytic cleaning destroys the tar, eliminating waste disposal problems. Potentially, catalytic cracking processes provide the simplest and most effective means of removing tars while retaining the sensible heat required for efficient use of producer gas in close-coupled applications. This work focuses on nickel-based catalysts treated with alkali in an effort to promote steam gasification of coke that deposits on the catalyst surface.

Since biomass gasification yields relatively high $\mathrm{CO} / \mathrm{H} 2$ ratios, higher $\mathrm{H} 2$ contents can be achieved by the water-gas shift reaction:

Eq. $1 \quad \mathrm{CO}+\mathrm{H}_{2} \mathrm{O} \leftrightarrow \mathrm{CO}_{2}+\mathrm{H}_{2} \quad \Delta \mathrm{H}=-41.1 \mathrm{KJ} \mathrm{mol}^{-1}$

This is usually achieved by operating two catalytic reactors in series: a high-temperature shift reactor for rapid reaction and a low-temperature shift reactor to shift thermodynamic equilibrium to very low levels of $\mathrm{CO}^{8}$. The high-temperature shift reaction takes advantage of faster kinetics at elevated temperatures to convert about $75 \%$ of the $\mathrm{CO}$ into $\mathrm{H} 2$. Since conversion is limited by thermodynamic equilibrium, which favors hydrogen formation at low temperatures, the gas is cooled before entering the second shift reactor.

Part of this study employed the combination of high-temperature and low-temperature water-gas shift reactors to convert $\mathrm{CO}$ to $\mathrm{H} 2$. However, during the last year of the project, a novel combined catalyst-sorbent system was investigated as a means of enhancing hydrogen yields in a single reactor system. To increase the $\mathrm{H} 2$ content, the hydrocarbons and carbon monoxide in the producer gas need to be converted to hydrogen via the steam reforming (SR) reaction and the water gas shift (WGS) reaction. Both the steam reforming and the water gas shift reaction can be limited by thermodynamic equilibrium as well as the rate of reaction. To overcome these limitations, a new material that combines a catalyst to increase the rate of reaction and a chemical sorbent for $\mathrm{CO} 2$ has been developed.

These core-in-shell catalyst-sorbent pellets, illustrated in Figure 3, consist of spherical pellets in which a $\mathrm{CaO}$ core (typically $3.4-4.0 \mathrm{~mm}$ in diameter) is surrounded by a porous but strong protective shell made of alumina (typically $0.3-0.5 \mathrm{~mm}$ in thickness) that supports a catalyst for both the steam reforming and the water gas shift reaction. During the hydrogen production period, the material serves two functions in catalyzing the reaction of hydrocarbons or carbon monoxide with steam to produce hydrogen while simultaneously absorbing carbon dioxide formed by the reaction. Efficient $\mathrm{CO}_{2}$ absorption is obtained at reaction temperature less than $600^{\circ} \mathrm{C}$ at $1.0 \mathrm{~atm}$ pressure. The in-situ removal of $\mathrm{CO}_{2}$ shifts the equilibrium of the steam reforming reaction and/or water gas shift reaction to increase the hydrogen concentration and simultaneously decreases the concentration of carbon oxides in the producer gas stream until the sorbent is saturated. The sorbent can be regenerated by simply heating the pellets (for instance by directly/indirectly passing flue gas) to $750^{\circ} \mathrm{C}$ to remove $\mathrm{CO}_{2}$. 


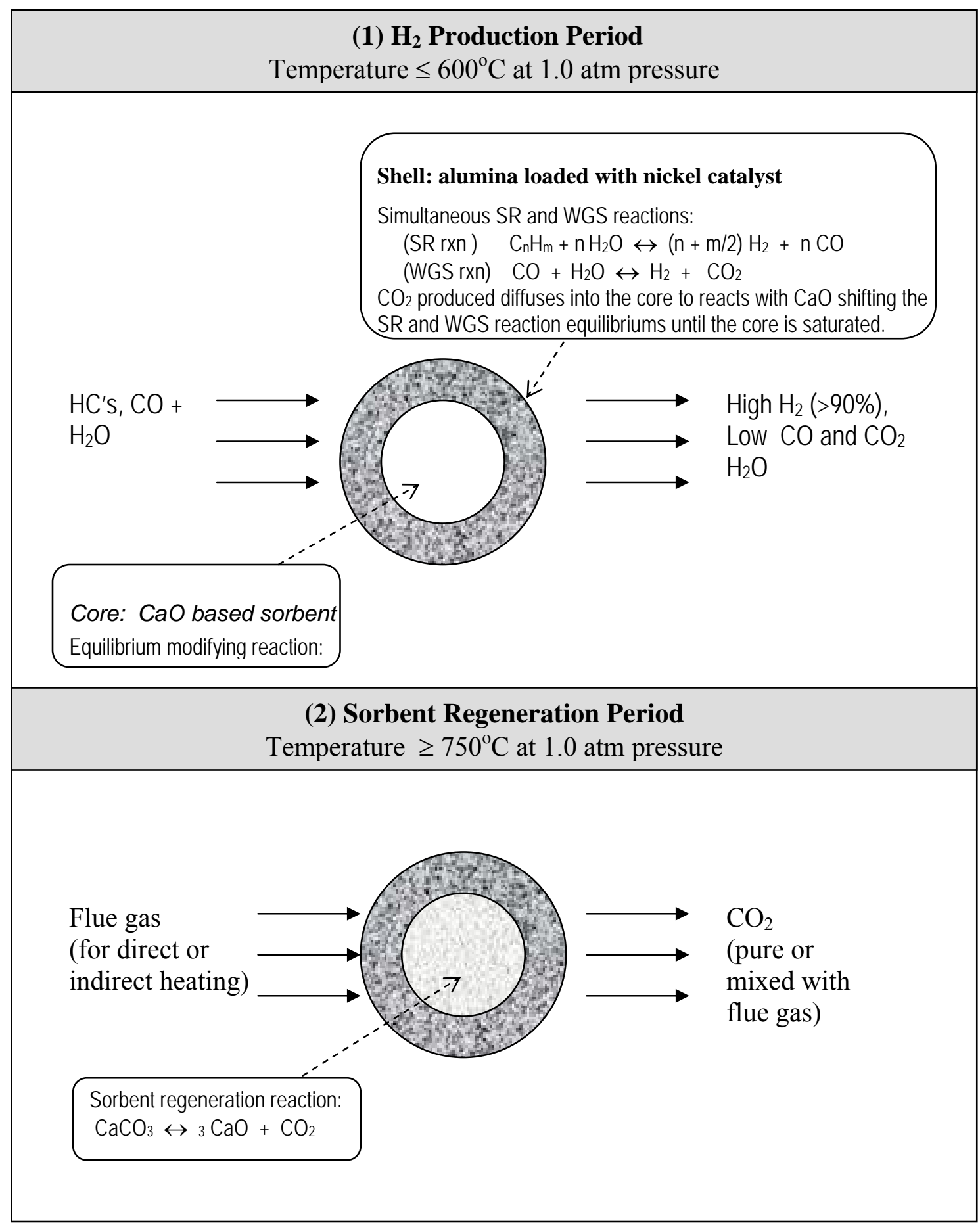

Figure 3 Schematic of a core-in-shell catalyst-sorbent pellet and reaction steps involved in producing high purity $\mathrm{H}_{2}$ from hydrocarbons and/or $\mathrm{CO}$. and in regenerating the sorbent. 
Goals and Approach of the Project

The goals of this project include:

- Determine whether switchgrass is a suitable fuel for the ballasted gasifier;

- Evaluate the usefulness of the moving bed granular filter for hot gas cleaning of producer gas from biomass gasifiers;

- Evaluate methods for measuring and removing contaminants from the producer gas prior to catalytic upgrading;

- Evaluate methods for mediating the water-gas shift reaction in the product gas;

- Model the thermal performance of the ballasted gasifier; and

- Estimate the economics of hydrogen production from switchgrass.

The approach to this project was to employ a pilot-scale ( 5 ton per day) gasifier to evaluate the thermally ballasted gasifier as a means for producing hydrogen from switchgrass. Gasification at the pilot scale was important for obtaining realistic process data, especially for calculating energy flows through the system and assessing the practicality of feeding switchgrass into the gasifier.

A slipstream from the gasifier was used to evaluate gas cleaning and upgrading options. This slip stream included particulate filtration equipment; a guard bed designed to remove hydrogen sulfide and hydrogen chloride and some tar; a steam reformer designed to crack the remaining tar and decompose ammonia; and high temperature and low temperature catalytic water-gas shift reactors to remove carbon monoxide from the product gas and increase its hydrogen content.

Other tests were operated on laboratory-scale equipment using simulated producer gas. These tests included evaluations of the combined catalyst/sorbent water-gas shift reactor and cold-flow trials of the moving bed granular filter.

2 Experimental Apparatus and Methods

\subsection{Biomass gasifier system}

A pilot-scale fluidized bed gasifier located at the Biomass Energy Conversion (BECON) Facility in Nevada, Iowa was used to gasify switchgrass and enhance the hydrogen content of the producer gas. The major components of the gasifier system include the fluid bed reactor, latent heat ballast, fluidization gas system, fuel delivery system, and data acquisition and control system. Figure 4 is a schematic showing the major components of the biomass gasifier system at BECON.

\section{Fluid bed reactor for primary conversion}

The fluidized bed reactor is capable of operating in combustion, gasification, or pyrolytic gasification modes. The ratio of air and fuel determine the mode of operation. In combustion mode the reactor is able to process approximately $20-60 \mathrm{~kg} / \mathrm{hr}$ of biomass. In gasification and 
pyrolytic gasification modes the reactor is able to process $90-270 \mathrm{~kg} / \mathrm{hr}$. The fluid bed is a bubbling, atmospheric pressure design. The dense phase of the bed is typically maintained at approximately $60 \mathrm{~cm}$. The bed has a diameter of $46 \mathrm{~cm}$ and a height of $3.3 \mathrm{~m}$. Thermocouples and pressure taps are located along the axis of the reactor to measure temperature and pressures, respectively.

\section{Latent heat ballast}

The latent heat ballasting system consists of an array of stainless steel tubes enclosing high temperature phase-change material. The array of tubes is immersed within the dense phase of the fluid bed, which assures high rates of heat transfer between tubes and biomass. The ideal phase change temperature would be close to the nominal combustion temperature of the fluidized reactor $\left(900^{\circ} \mathrm{C}\right)$ and have large heat of fusion on a volumetric basis. Of course, the material should be non-flammable and relatively inexpensive. A large number of high temperature phasechange materials were surveyed for this application ${ }^{9}$. Our work to date has employed lithium fluoride (LiF), a stable salt that does not react with stainless steel, as ballast material. Lithium fluoride has an ideal melting point $\left(848{ }^{\circ} \mathrm{C}\right)$, high latent heat of fusion $(1050 \mathrm{~kJ} / \mathrm{kg})$, and high volumetric heat capacity $\left(1800 \mathrm{MJ} / \mathrm{m}^{3}\right)$.

Ballast tubes were constructed of $25.4 \mathrm{~mm}$ diameter stainless steel tubes of $610 \mathrm{~mm}$ length. Each of the ballast tubes was filled with $0.3 \mathrm{~kg}$ of LiF. An air pocket was left in each of the tubes to allow for expansion of the LiF. Forty-eight ballast tubes, covering about $15 \%$ of the bed cross sectional area, were used during the tests in the fluidized bed gasifier. This represents a total latent heat storage capacity of $15,100 \mathrm{~kJ}$. The completed assembly is in shown in Figure 5.

\section{Fluidization gas system}

Air may be used to fluidize the bed and supply oxygen for combustion or gasification modes. A positive displacement blower supplies air to the fluid bed reactor distributor. House compressed air supplies purge air to the material handling system. Steam may be co-injected with air or may be used to fluidize the bed during pyrolytic gasification. A boiler generates saturated steam at flow rates of $0-180 \mathrm{~kg} / \mathrm{hr}$. A $40 \mathrm{~kW}_{\mathrm{e}}$ circulation heater may be used to superheat the steam to $550{ }^{\circ} \mathrm{C}$.

\section{Fuel delivery system}

Two feed hoppers and metering screw augers are used to supply biomass to the fluid bed reactor. Wide variations in fuel properties require the two feed systems. One system is dedicated to metering shelled corn, which is uniform in size and density and flows easily. The other system, labeled "fibrous" in Figure 4, is used for all other feedstocks including switchgrass. Switchgrass must be ground to a maximum particle size of $30 \mathrm{~mm}$ to facilitate uniform metering to the fluid bed.

Rotary airlocks form a pressure barrier between the slightly positive pressure (12 kPa gage) fluid bed reactor and atmosphere. The airlocks are not completely air tight, necessitating purge gas 


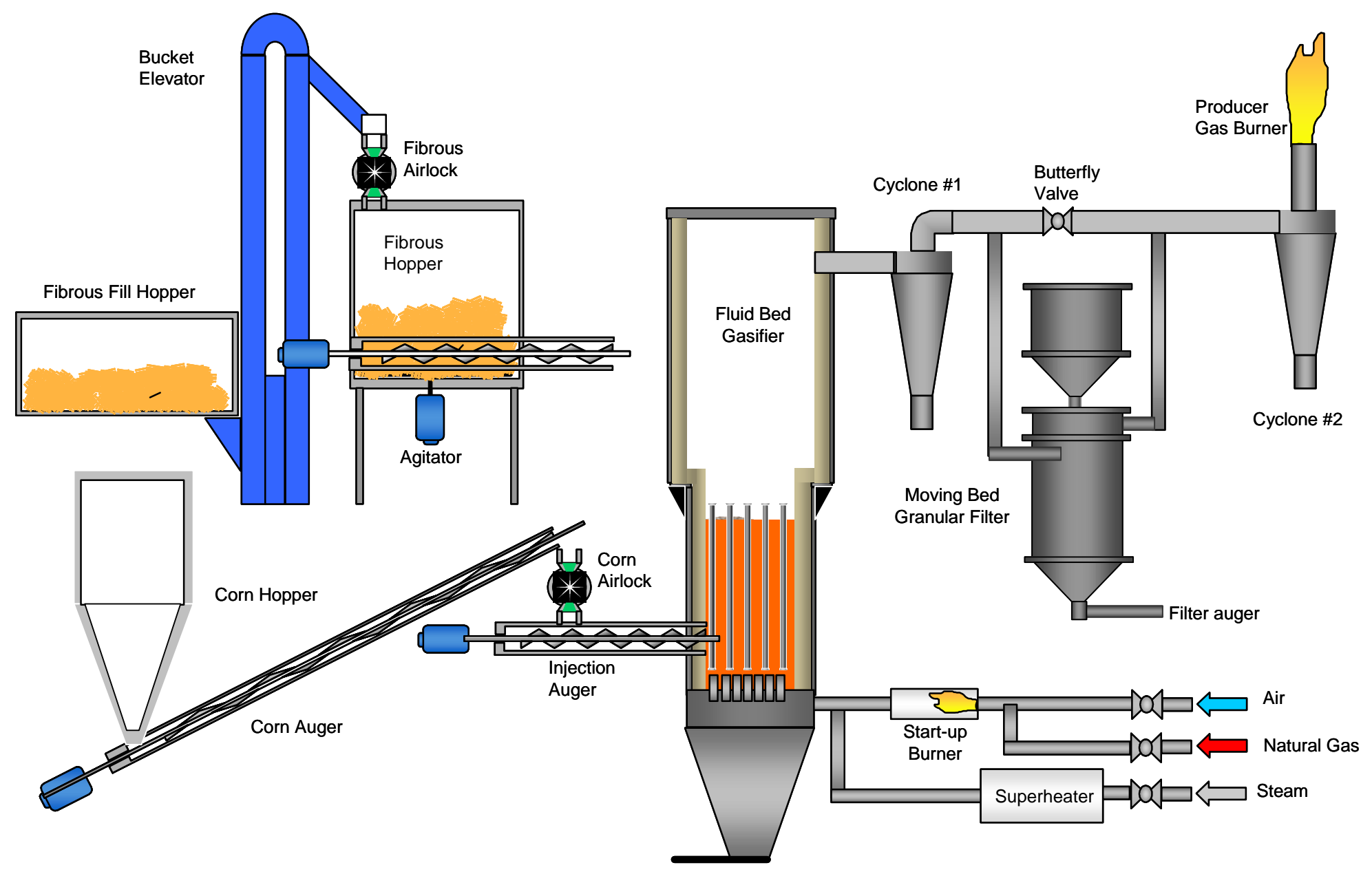

Figure 4. Schematic of the fluid bed conversion system 


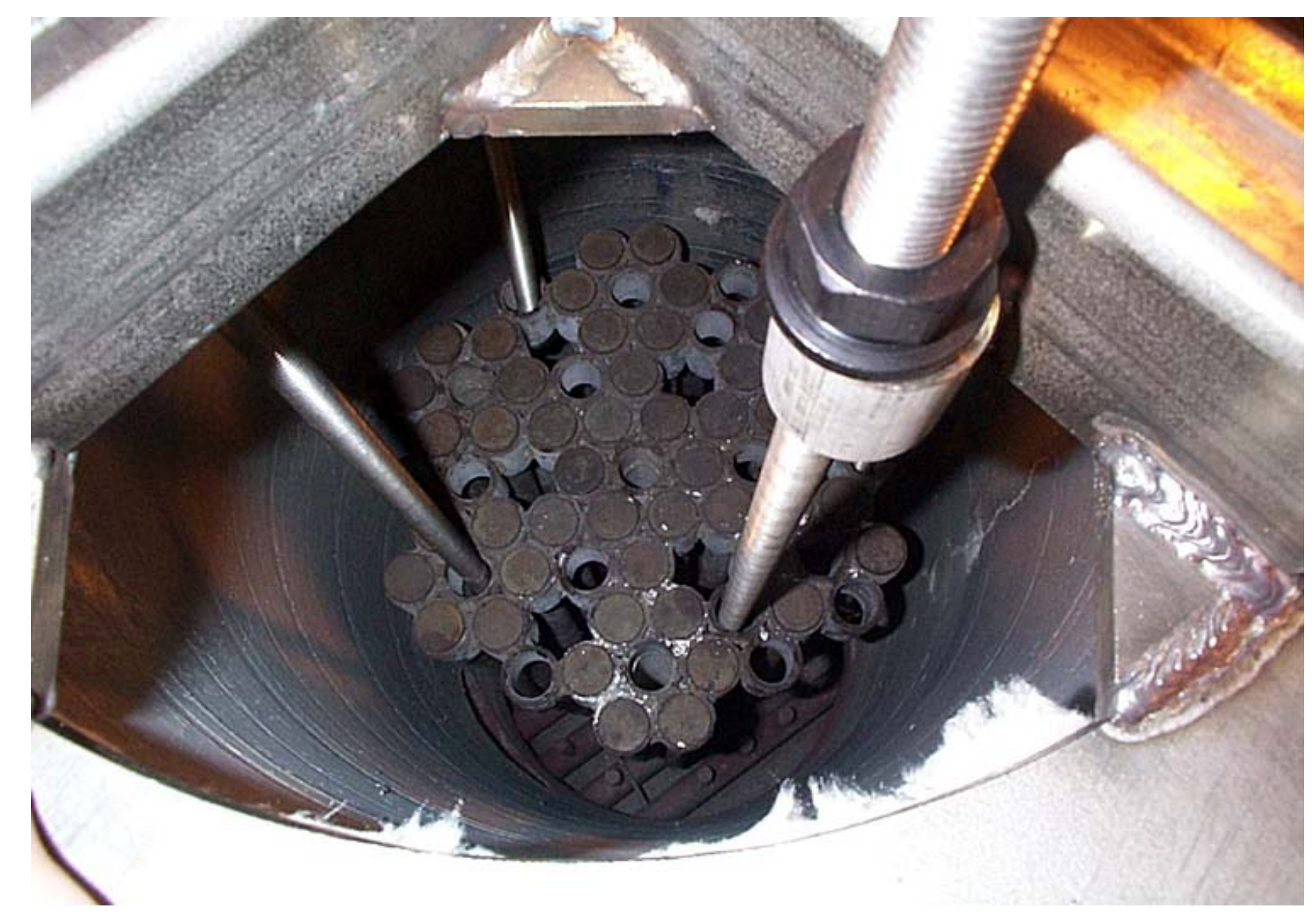

Figure 5 Overhead view of latent heat ballast system installed in 5 tpd biomass gasifier 
(air or nitrogen) on the feed system to prevent the back flow of hot reaction products. The corn and fibrous systems expel biomass to a high-speed injection auger that carries the material into the fluid bed reactor.

\section{Data acquisition and control system}

The data acquisition and control system consists of Allen-Bradley ${ }^{\mathrm{TM}}$ SLC 500 rack (10 slot) with modules for thermocouple input, analog input, digital input, analog output, and digital output. Wonderware $^{\mathrm{TM}}$ V7.0 software is used for the graphical user interface.

The control system incorporates all of the data and control points on the gasification system. This system allows five modes of operation: start-up, auto-combustion, auto-gasification, autoballasted gasification, and shutdown. The modes will automate much of the process enabling minimal input from users during operation. For example, the auto-combustion mode uses PID control to vary the fuel feed rate to achieve a user-specified bed temperature. The auto-ballasted gasification mode will automatically control the air, steam, and fuel feed rates as the system cycles between combustion and pyrolysis. The control system also monitors all data inputs and shuts down the system if an input goes out of range and/or notifies the operator of an alarm or shutdown condition.

\section{Method of operation}

The gasifier system is initiated by selecting the start-up mode. A natural gas burner preheats the fluidization air to heat the reactor to solid fuel combustion temperatures, usually $300^{\circ} \mathrm{C}$. Small quantities of biomass are added to the reactor until the temperature reaches $700^{\circ} \mathrm{C}$, when the system switches into auto-combustion mode and the natural gas preheater is secured. At this point the operator may choose to continue operation in auto-combustion mode, switch to autogasification mode (air-blown gasification), or switch to auto-ballasted gasification mode. Depending on the mode of operation, the control system will vary the input based on user defined set points.

Ballasted gasification involves alternating periods of combustion and pyrolysis. The initial mode of operation is combustion to bring the fluid bed and ballast system to a temperature greater than $900{ }^{\circ} \mathrm{C}$. Table 1 shows typical fluid bed operating parameters for the combustion and pyrolysis phases. The air flow rate and fuel feed rate during the combustion phase are $4.0 \mathrm{Nm}^{3} / \mathrm{min}$ and 55 $\mathrm{kg} / \mathrm{hr}$. The pyrolysis phase is initiated by securing the air flow, initializing steam flow, and adjusting the fuel feed rate. The steam and fuel rates are $90 \mathrm{~kg} / \mathrm{hr}$ and $180 \mathrm{~kg} / \mathrm{hr}$, respectively. The reactor temperature was allowed to decrease (due to endothermic reaction) to approximately $650{ }^{\circ} \mathrm{C}$ at which time combustion is reinitiated and the cycle repeated. Subsequent combustion cycles are split into a char burnout phase and fuel combustion phase. At the close of the pyrolysis cycle significant quantities of char remain in the fluid bed reactor. This char is combusted prior to initializing the combustion fuel feed rate. 
Table 1. Fluid bed operating parameters during combustion and pyrolysis cycles of ballasted gasification experiments.

\begin{tabular}{|c|c|c|c|}
\hline Parameter & Unit & Combustion & Pyrolysis \\
\hline Air flow rate & $\mathrm{m}^{3} / \mathrm{min}$ & 4.0 & 0.0 \\
\hline Fuel flow rate & $\mathrm{kg} / \mathrm{hr}$ & 55 & 225 \\
\hline Steam flow rate & $\mathrm{kg} / \mathrm{hr}$ & 0.0 & 90 \\
\hline Bed temperature & ${ }^{\circ} \mathrm{C}$ & $650-900$ & $900-650$ \\
\hline
\end{tabular}

\subsection{Moving Bed Filter}

\section{Theoretical Background}

Granular filtration is a fluid-solid separation process where suspended solid particles are removed from a flowing fluid stream. Particles are removed from the fluid as the flow is forced through a bed of granular media. Various collection mechanisms act on suspended particles causing them to interact with and be collected by the granular media. Collection mechanisms of inertial impaction, interception, diffusion, sedimentation and straining may all act to collect suspended particles.

For granular beds composed of particles larger than $1 \mathrm{~mm}$ and removing particles smaller than $50 \mu \mathrm{m}$, inertial impaction is generally considered to be the most important filtration mechanism. Inertial impaction is characterized by the dimensionless Stokes number, $S t$ :

Eq. $2 \quad S t=\frac{\rho_{p} \cdot d_{p}^{2} \cdot U \cdot C_{s}}{9 \cdot \mu \cdot d_{g}}$

where $\rho_{p}$ is the dust particle density, $d_{p}$ is the dust particle diameter, $\mu$ is the fluid viscosity, $d_{g}$ is the granule diameter, $\mathrm{Cs}$ is the Cunningham correction factor for velocity slip at the particle surface, and $\mathrm{U}$ is the superficial velocity at the face of the filter media.

The performance of a granular filter is commonly quantified by particle collection efficiency $(\eta)$ :

Eq. 3

$$
\eta=\frac{m_{\text {in }}-m_{\text {out }}}{m_{\text {in }}}
$$

where $m_{\text {in }}$ is the mass of dust particles entering the filter and $m_{\text {out }}$ is the mass of dust particles exiting the filter.

The mass balance on a moving bed filter is given by:

Eq. 4

$$
\frac{\partial C}{\partial t}+U \frac{\partial C}{\partial x}=R
$$

where $\mathrm{C}$ is the concentration of dust in the gas flow, $\mathrm{U}$ is the superficial velocity of gas through the granular bed, $\mathrm{R}$ is the rate of removal of dust from the gas stream, and $\mathrm{x}$ and $\mathrm{t}$ are the spatial and temporal variables for this one-dimensional formulation of the filter. In general, the dust 
removal rate will be a complex function of both dust concentration and the instantaneous loading of dust in the filter. However, for relatively low dust loadings in the filter, this rate can be assumed to be first-order with respect to dust concentration:

Eq. $5 \quad R=-k C$

where $\mathrm{k}$ is the rate constant for dust removal by the filter. Since the moving bed filter can be operated in steady state, Eq. 4 simplifies to:

Eq. $6 \quad U \frac{\partial C}{\partial x}=-k C$

Integrating this expression over the height of the bed, L, and combining it with Eq. 3 yields:

Eq. 7

$$
\eta=1-\exp (-k L / U)
$$

Often it is convenient to express filter performance in terms of penetration, $\mathrm{P}$, which is defined as $1-\eta:$

Eq. $8 \quad P=\exp (-k L / U)$

This expression suggests that filter performance should correlate with the parameter L/U, which is equivalent to the gas residence time in the filter. However, $k$ is proportional to the "single layer" collection efficiency of granules in the bed ${ }^{10}$, which is typically dependent on Stokes number. Thus, $k$ may contain an implicit dependence on $U$ in addition to the explicit dependence found in the $L / U$ parameter. Brown ${ }^{10}$ and Amundson ${ }^{11}$ have developed a similar expression for removal of substances from fluid streams.

Two kinds of particulate filtration experiments on moving granular beds were performed: hot gas and cold gas tests. The hot gas tests had the advantage of operating under realistic conditions of biomass gasification, but obtaining accurate and precise data on filter performance was difficult. For this reason, cold gas tests on a laboratory-scale filter were also performed, which allowed more comprehensive investigations of the mechanisms of filtration.

\section{$\underline{\text { Hot Gas Filtration }}$}

Hot gas filtration trials were performed with a pilot-scale moving bed granular filter installed downstream of the $5 \mathrm{tpd}$ fluidized bed gasifer used for ballasted gasification development. The nominal gas generation rate is $340 \mathrm{~nm}^{3} \mathrm{~h}^{-1}$.

The experiments employed a mixture of waste seed corn and waste soybean seed as fuel, which are waste streams of interest to one segment of the agricultural processing industry. The bed of sand and limestone was fluidized with air at an equivalence ratio between 0.25 and 0.30 , which maintained the bed in the temperature range of 700 to $760{ }^{\circ} \mathrm{C}$. The feed rate of biomass during these trials was in the range of $150-180 \mathrm{~kg} / \mathrm{h}$. 
The filter, illustrated in Figure 2 and Figure 6, consists of five major sections: a cyclonic inlet, a flow straightening section, an interfacial gas contacting region, a granule downcomer, and a gas disengagement section. The cyclonic gas inlet imparts a radial component to the gas flow for the purpose of reducing entrance pressure losses. This inlet consists of a $0.914 \mathrm{~m}$ diameter cylinder of $0.762 \mathrm{~m}$ length constructed of mild steel. Once inside the MBGF, the hot gas swirls radially downward until it reaches a flow straightening section. This section serves to redirect the gas uniformly and perpendicularly into the gas-contacting region while preventing reentrainment of deposited particulate. Each of the 80 flow straighteners measure $0.076 \mathrm{~m}$ long and $0.07 \mathrm{~m}$ wide. Particulate is filtered from the gas flow in this interfacial region. After passing through the interfacial region, the clean gas flows upward through the granule down-comer until it reaches the disengagement section. The disengagement section consists of a $0.76 \mathrm{~m}$ diameter cylinder of $0.61 \mathrm{~m}$ length constructed of mild steel. A stainless steel mesh screen retains granular material and prevents the moving bed from fluidizing. Without this screen, granules would tend to be entrained in the exiting gas stream. Further details are found in Brown et al. ${ }^{12,13}$ The constructed filter, before and after installation, is shown in Figure 7.

Granular material is gravity fed to the filter from a feed hopper above the filter by means of a $0.07 \mathrm{~m}$ dia. delivery pipe that passes through the center of the mesh screen (gas does not flow into the delivery pipe because the feed hopper is sealed). The capacity of the filter is $0.45 \mathrm{~m}^{3}$ while that of the hopper is $0.73 \mathrm{~m}^{3}$. Dust-laden granular material exiting the bottom of the filter is augured into a barrel for subsequent disposal. Granular material used for testing were $3 \mathrm{~mm}$ dia., smooth (non-porous) silica pebbles obtained from American Materials Corp. of Eau Claire, Wisconsin.

The sampling systems upstream and downstream of the MBGF (designated as the inlet and exit sampling systems, respectively) are designed to separately capture particulate and tar as well as measure gas composition. As subsequently described in Section 2.4, each sampling system consists of a sample probe, a heated sintered-metal particulate filter, an impinger train to collect tar, a vacuum pump with a bypass control valve, and a rotameter. Additionally, gas exiting the vacuum pumps can be directed to a Varian Model CP2003 Micro-Gas Chromatograph for determination of gas composition.

\section{Cold Flow Experiments}

The experimental apparatus used to evaluate the moving bed granular filter under cold flow conditions is illustrated in

Figure 8. A compressed air source provided gas flow in the range of 425 to $700 \mathrm{~L} / \mathrm{min}$ to the filter through a globe valve and a variable area flow meter with a range of 0 to $1150 \mathrm{~L} / \mathrm{min}$. Dust was injected into the air stream using a sealed Shenck-Accurate model MOD106M bulk solids material feeder. Nominal dust injection rates were $1 \mathrm{~kg} / \mathrm{h}$, but were influenced by gas flow rates past the injection point. Preliminary tests revealed that filtration efficiency was not influenced by the resulting variations in inlet dust concentrations, so no attempt was made to strictly control these injection rates. Total mass of dust injected into the moving bed granular filter was calculated as the difference in weight before and after the test. 
The moving bed granular filter, designed along the lines shown in Figure 8, consisted of a 19.1 $\mathrm{cm}$ dia. filter body enclosing a $15.2 \mathrm{~cm}$ dia. downcomer of height $18 \mathrm{~cm}$. Granules flowed downward through the filter by gravity at a rate controlled by a variable speed auger at the bottom of the filter. The dirty granular media was captured in a sealed collection vessel below the filter. Red Flint gravel with an average particle size of $4 \mathrm{~mm}$ was used as the granular media. The nominal granule flow rate was $4 \mathrm{~kg} / \mathrm{h}$.

The gas flow exiting the granular bed passed through a pleated HEPA filter sealed in a dust collection box. A Setra model 1000 scale accurate to $\pm 0.05 \mathrm{~g}$ was used to determine the weight of dust collected in the HEPA filter. Dust deposited in the collection box was also collected and weighed as part of the total mass of dust exiting the moving bed granular filter. Dust collection efficiency for a test was calculated according to Eq. 3. Uncertainty analysis typically indicated the efficiency to be accurate within $0.05 \%$. Most tests were performed with coal fly ash obtained from a local power plant that has a bulk density of $1260 \mathrm{~kg} / \mathrm{m}^{3}$. The size distribution for this fly ash is given in Figure 9. The calculated average size of these particles is $14 \mu \mathrm{m}$ although it should be noted from the size distribution that the particles are bimodally distributed.

\subsection{Catalytic Upgrading System}

Catalytic upgrading is intended to reform tar to $\mathrm{CO}$ and $\mathrm{H}_{2}$ and water-gas shift $\mathrm{CO}$ to additional hydrogen. Two different approaches to this process were investigated. The first employed a conventional catalytic system of separate reactors for steam reforming and two stages of watergas shift reaction. The second employed a combined catalysis/sorbent system for this purpose.

\section{Conventional Catalytic System}

A nominal $5 \mathrm{~L} \mathrm{~min}^{-1}$ slipstream from the gasification stream was used to evaluate gas cleaning and hydrogen enhancement. This catalytic reaction system, illustrated in Figure 10, included a guard bed; a tar (steam reforming) reactor; and high temperature and low temperature catalytic water-gas shift reactors. The slipstream passed through a heated particulate filter before entering the catalytic reaction system. Both the slipstream line and the particulate filter were maintained at $450^{\circ} \mathrm{C}$ to prevent condensation of tars. The slipstream entered each reactor from the top and exited from the bottom before passing to the next reactor.

The four fixed-bed reactors were of identical construction, fabricated from $22 \mathrm{~mm}$ ID stainless steel pipe, and can be filled to various depths to give space velocities between $1500 \mathrm{~h}^{-1}$ and 6000

$\mathrm{h}^{-1}$. Each was mounted in an electrically heated oven to maintain desired temperatures for the experiments. Each reactor, as illustrated in Figure 11, had two thermocouples: one at the center of the fixed bed, which was moveable for obtaining longitudinal temperature profiles, and the other fixed at the center of catalytic bed, which was used for temperature control.

The first reactor, the guard bed, was designed to capture fine particulate, absorb sulfur and chloride contaminants, and provide partial steam reforming of tars. It played an important role in protecting the metal catalysts in the reactors downstream of it, which are susceptible to coking by heavy tars and poisoning by sulfur and chlorine species. Calcined dolomite was used in the 

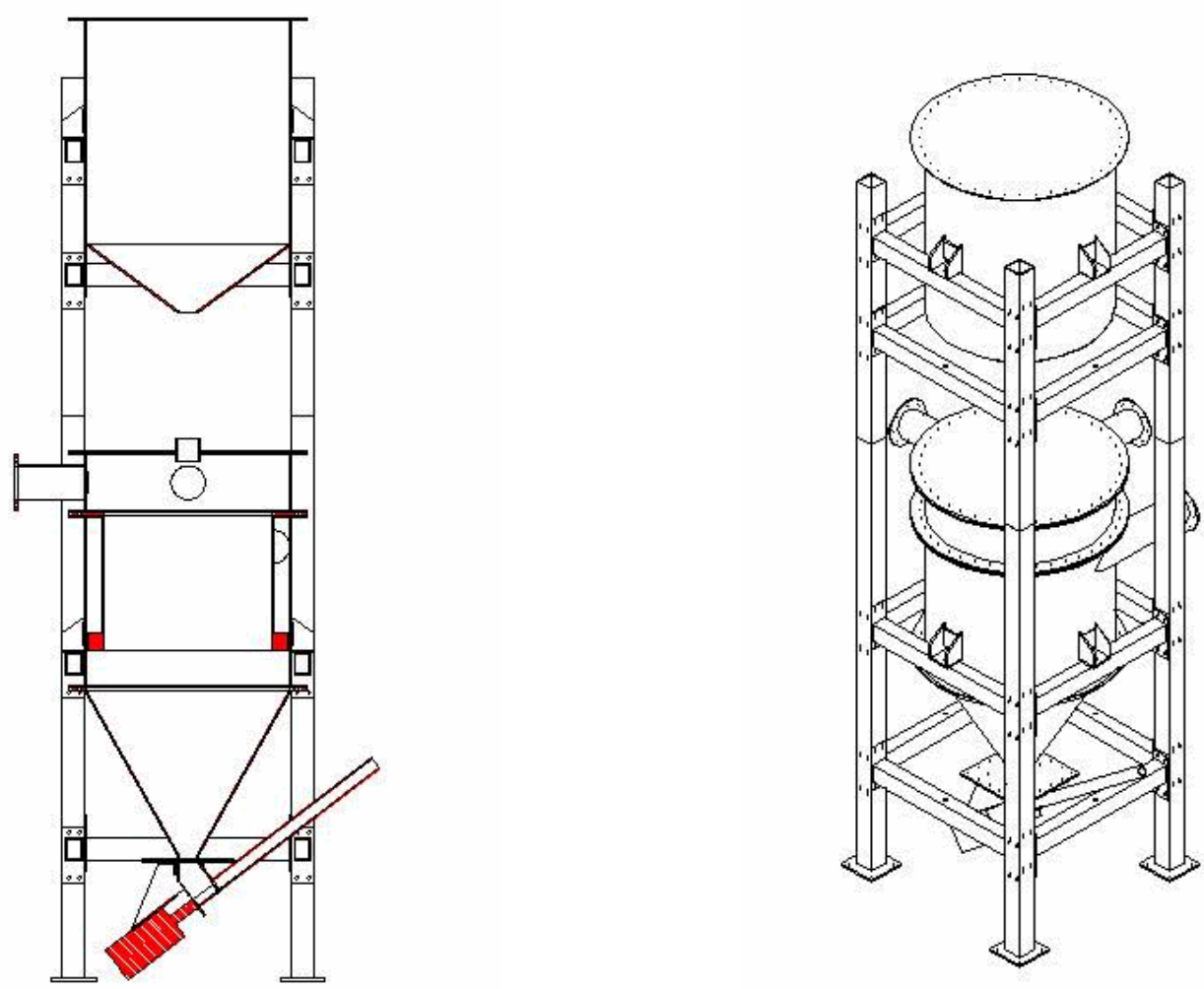

Figure 6 Cross-sectional and Iso-metric view of moving bed granular filter 
(a)

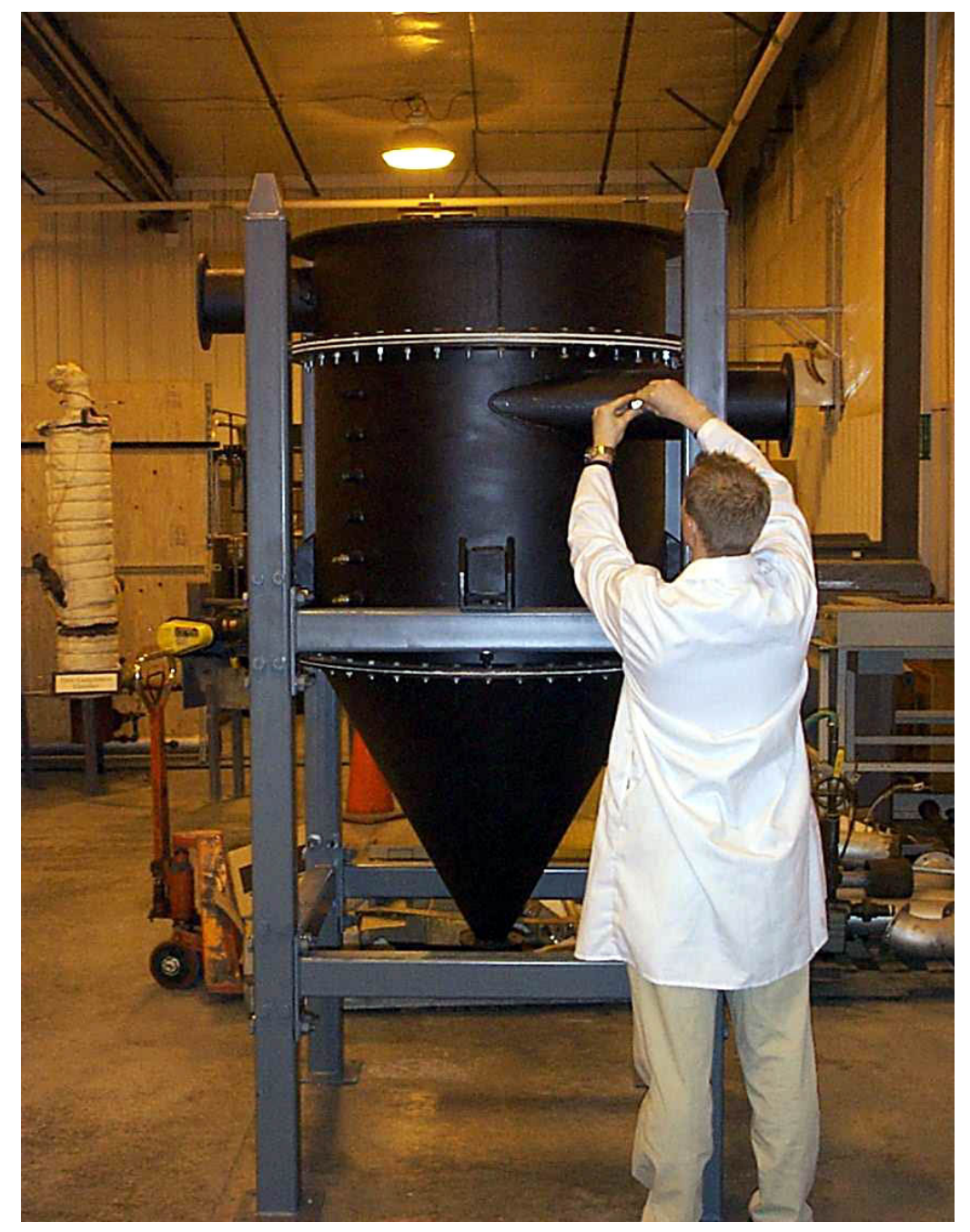

(b)

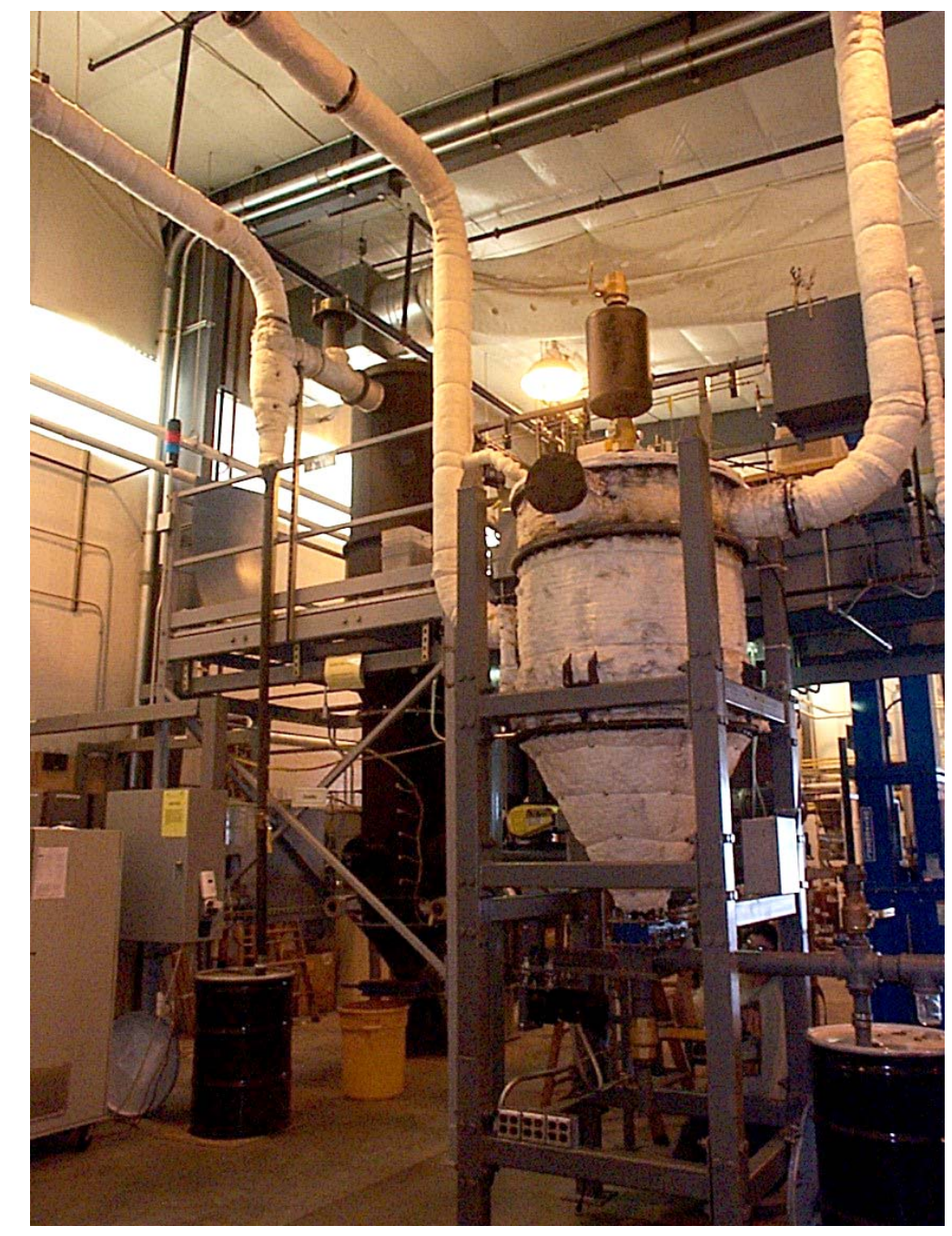

Figure 7 Photographs of the moving bed granular filter (a) after construction, (b) after installation 


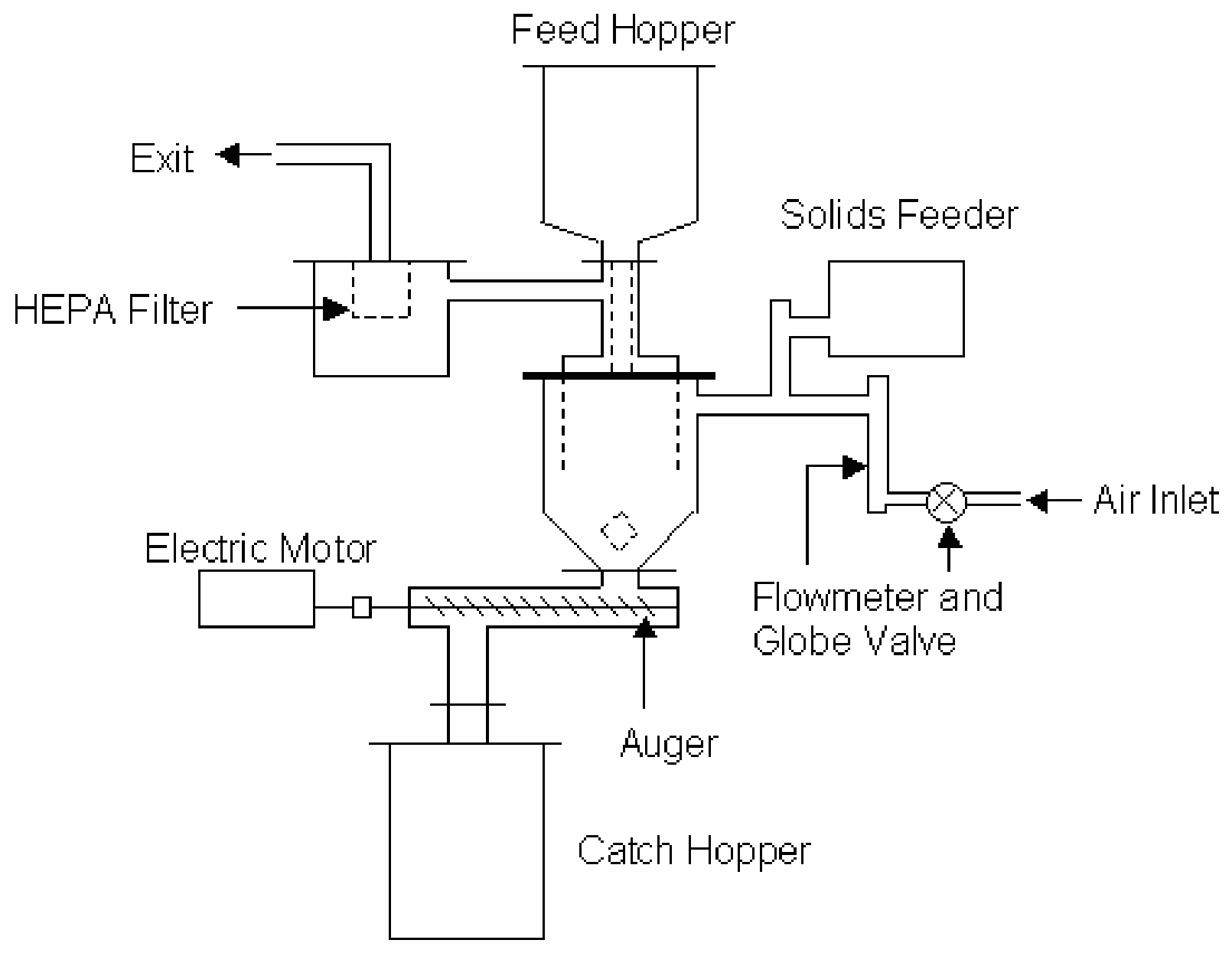

Figure 8 Experimental apparatus 


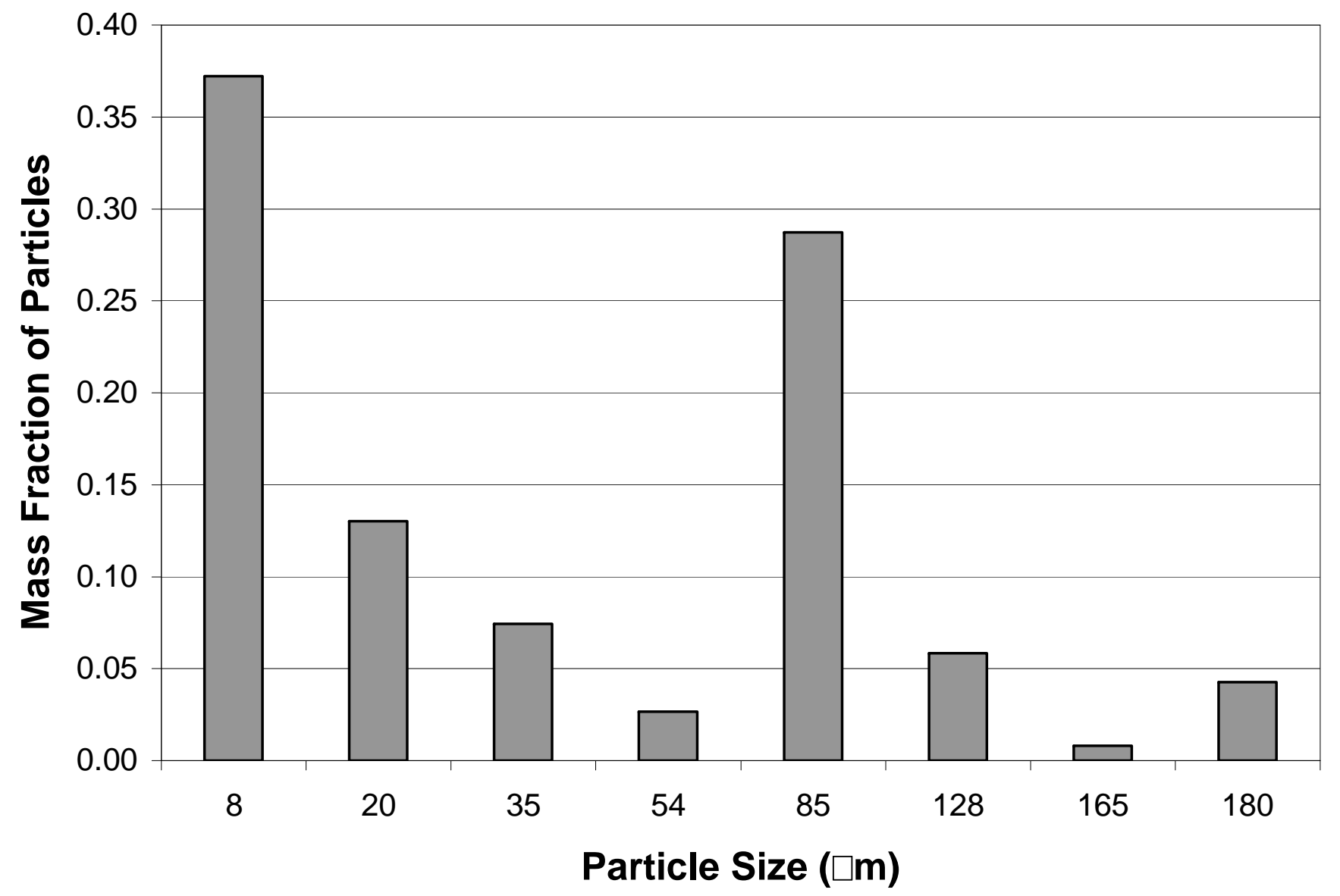

Figure 9 Size distribution of fly ash 


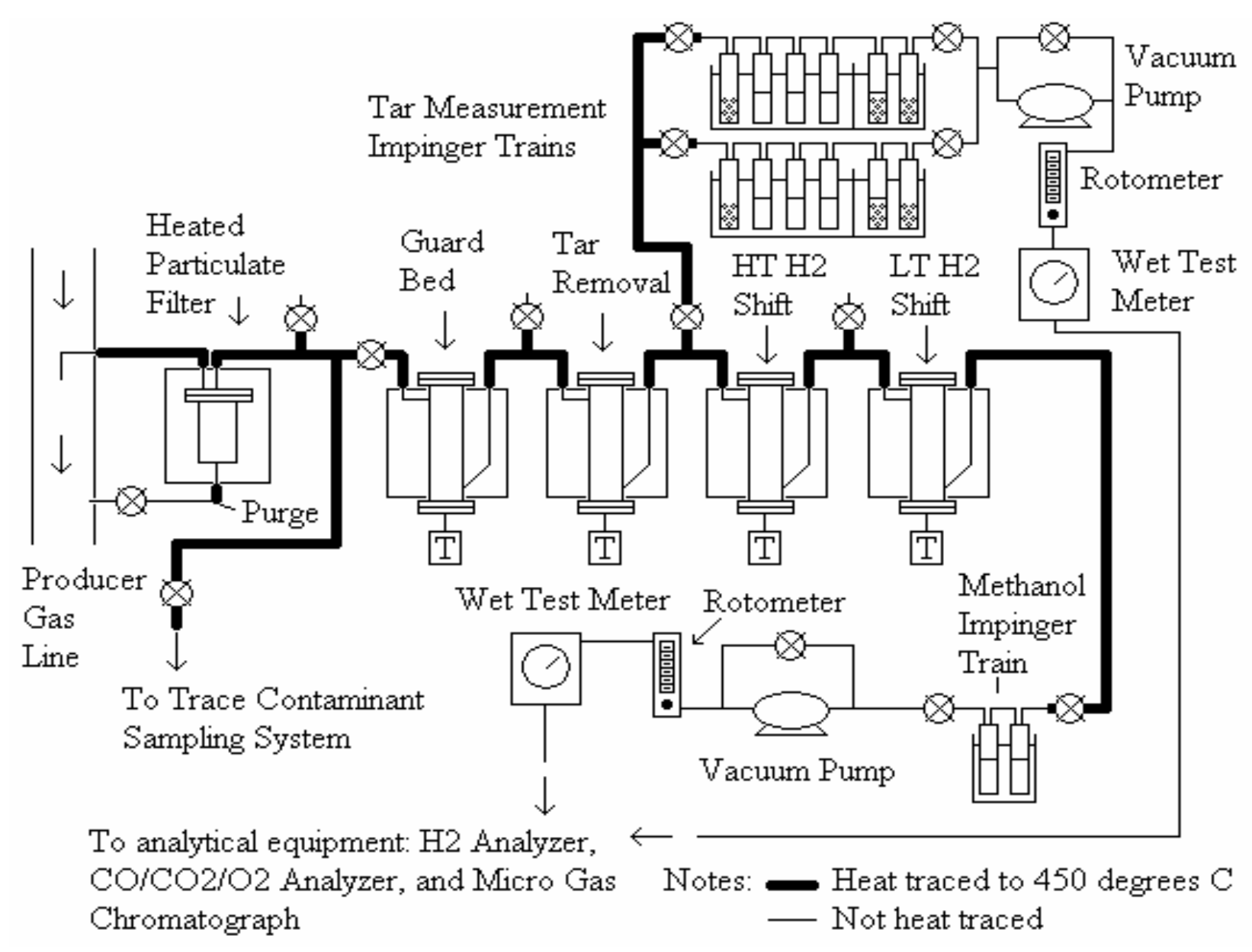

Figure 10 Schematic of overall experimental apparatus 


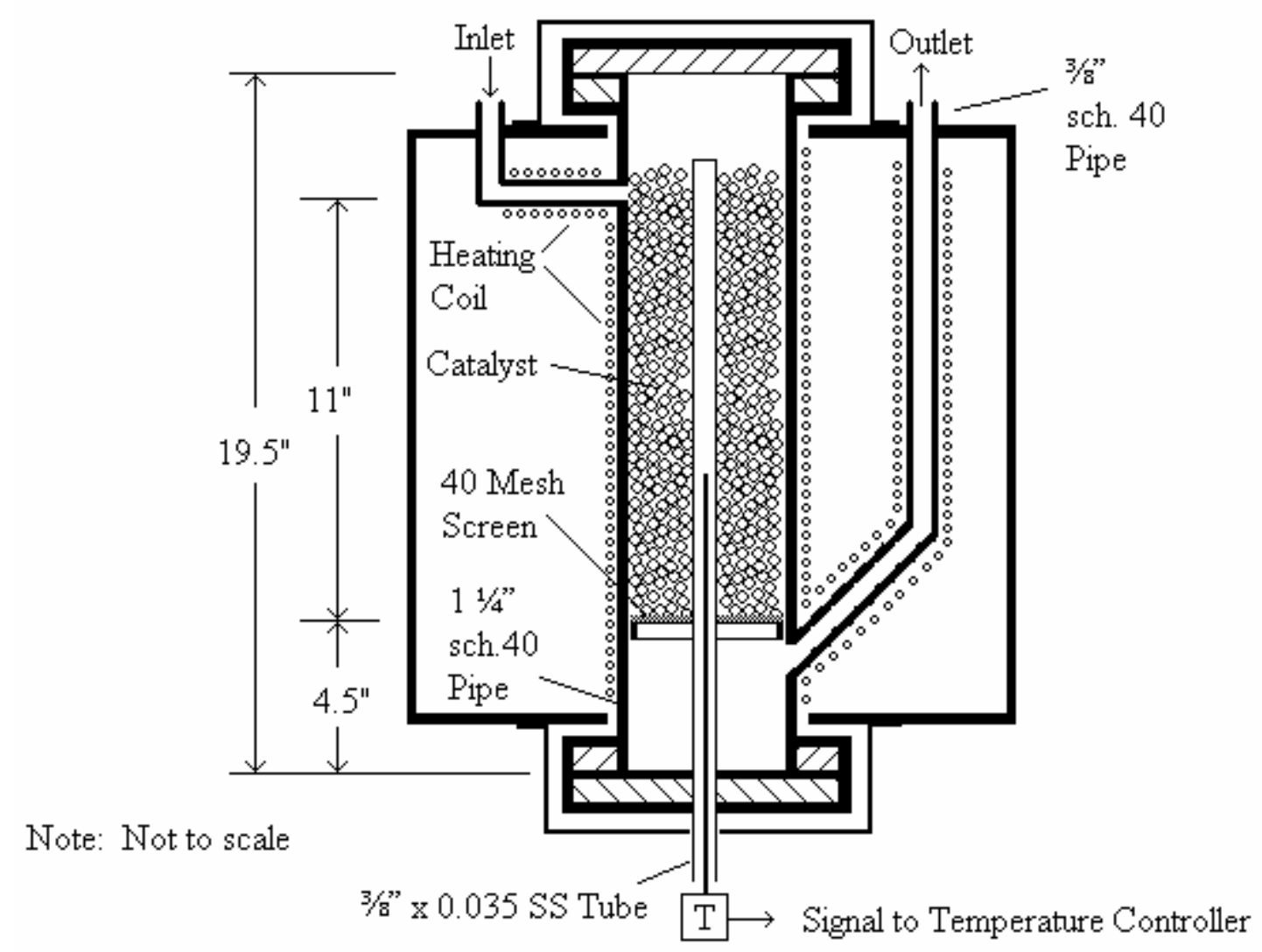

Figure 11 Drawing of catalytic reactor 
guard bed reactor. No attempt was made to regenerate the dolomite, which is an inexpensive sorbent.

The second reactor is designed to steam reform tars to $\mathrm{CO}$ and $\mathrm{H}_{2}$. Three steam-reforming, Nibased catalysts were evaluated in the tar reactor: ICI46-1 was produced by the Imperial Chemical Industry while Z409 and RZ409 are products of Qilu Petrochemical Corp., P.R. China. The compositions of these catalysts are listed in Table 2. All three catalyst contained alkali additives, such as potassium, calcium and magnesium oxides, which promote the elimination of coke formed on the catalyst. Although the potassium promoter might be expected to readily diffuse out of the catalyst, it is in the form of potassium aluminosilicate, which releases the potassium very slowly, resulting in long service life.

The steam-reforming catalysts are usually activated before use by exposure to a reducing environment, typically a mixture of $\mathrm{N}_{2}$ and $\mathrm{H}_{2}$ at $750-850^{\circ} \mathrm{C}$ for several hours. However, in our experiments, we tested ICI46-1 and Z409 without reduction. RZ409 is a reduced form of Z409 prepared by the manufacturer. The as-received catalysts were in the form of $15 \mathrm{~mm}$ rings. For use in our reactor these rings were crushed and sifted to obtain 0.9-2.0 mm diameter particles. The pore size distributions of the crushed and sieved catalyst particles were obtained by mercury porosimetry. Typical characteristics for catalysts used in steam reforming are: specific surface area of $16-23 \mathrm{~m}^{2} / \mathrm{g}$; total pore volume of $0.14-0.18 \mathrm{~cm}^{3} / \mathrm{g}$; and average pore diameter of 200 $500 \AA$.

The operating conditions for tests designed to evaluate the performance of the steam reforming catalysts are given in Table 3 . In addition to the type of catalysts, operating variables include temperature of the guard bed $\left(\mathrm{T}_{\mathrm{GB}}\right)$, temperature of the steam reforming reactor $\left(\mathrm{T}_{\mathrm{SR}}\right)$, space velocity (SV) calculated on a dry gas basis, and the ratio of steam to total organic carbon ratio (steam/TOC). Total organic carbon represents the amount of carbon in the organic compounds that are susceptible to steam reforming. Steam for steam reforming was generated by pumping water through a micro-pump into a stainless steel pipe heated to $150-240{ }^{\circ} \mathrm{C}$, which was admitted ahead of the guard bed reactor.

For the water-gas shift reactors, Fe-Cr based catalyst was used for the high temperature reactor while a $\mathrm{Cu}-\mathrm{Zn}$ based catalyst was used in the low temperature reactor. The catalyst for the high temperature reactor is used commercially in fertilizer factories in China and is manufactured according to China National Patent No. ZL 96102477.1. ${ }^{14}$ The catalyst for the low temperature reactor is designated as B202. The chemical compositions of these shift catalysts are given in Table 2. The catalysts are commercial products of cylindrical shape with diameters and heights on the order of several millimeters, as received. For use in the reactors they are crashed to small amorphous particles of $0.6-2.0 \mathrm{~mm}$ diameter. The catalysts were sandwiched between layers of inert alumni spheres ( $4 \mathrm{~mm}$ diameter) in the reactors.

The shift catalysts were reduced prior to evaluation of catalytic activity. This was done by treating them with producer gas in the amount of $1 \mathrm{~L} / \mathrm{min}$ along with steam injected at a steam/gas ratio of 0.8 . The Fe-Cr based catalyst in the high temperature shift reactor was reduced at $250^{\circ} \mathrm{C}$ and the $\mathrm{Cu}-\mathrm{Zn}$ based catalyst in the low temperature shift reactor was reduced 
Table 2 Chemical composition of catalysts

\begin{tabular}{|l|l|l|l|c|l|}
\hline Catalyst & $\begin{array}{l}\text { Active } \\
\text { Component }\end{array}$ & Promoter & Carrier & Binder & $\begin{array}{l}\text { Preparati } \\
\text { on }\end{array}$ \\
\hline $\begin{array}{l}\text { Steam reforming } \\
\text { ICI46-1 }\end{array}$ & $\mathrm{NiO}$ & $\mathrm{CaO}, \mathrm{K}_{2} \mathrm{O}$ & $\mathrm{SiO}_{2}, \mathrm{Al}_{2} \mathrm{O}_{3}$ & - & $\begin{array}{l}\text { Not } \\
\text { reduced }\end{array}$ \\
\hline $\begin{array}{l}\text { Steam reforming } \\
\mathrm{Z} 409\end{array}$ & $\mathrm{NiO}$ & $\mathrm{MgO}, \mathrm{K}_{2} \mathrm{O}, \mathrm{FeOx}$ & $\mathrm{SiO}_{2}, \mathrm{Al}_{2} \mathrm{O}_{3}$ & - & $\begin{array}{l}\text { Not } \\
\text { reduced }\end{array}$ \\
\hline $\begin{array}{l}\text { Steam reforming } \\
\text { RZ409 }\end{array}$ & $\mathrm{NiO}$ & $\mathrm{MgO}, \mathrm{K}_{2} \mathrm{O}, \mathrm{FeOx}$ & $\mathrm{SiO}_{2}, \mathrm{Al}_{2} \mathrm{O}_{3}$ & - & Reduced \\
\hline $\begin{array}{l}\text { High temperature } \\
\text { shift }\end{array}$ & $\begin{array}{l}\mathrm{Fe}_{2} \mathrm{O}_{3} \\
78 \pm 2 \%\end{array}$ & $\begin{array}{l}\mathrm{Cr}_{2} \mathrm{O}_{3} 9 \pm 2 \% ; \mathrm{CuO} 2.0 \% ; \mathrm{Rare} \\
\text { earth } 1.5 \%\end{array}$ & - & $\begin{array}{l}\text { Carbon } \\
\text { black } 0.5 \%\end{array}$ & Reduced \\
\hline $\begin{array}{l}\text { Low temperature } \\
\text { shift }\end{array}$ & $\mathrm{CuO}>29 \%$ & $\mathrm{ZnO} 41-47 \% ; \mathrm{Al}_{2} \mathrm{O}_{3} 8.1-10 \%$ & - & - & Reduced \\
\hline
\end{tabular}


Table 3 Operating conditions for steam reforming catalysts

\begin{tabular}{|c|c|c|c|}
\hline Parameter & ICI 46-1 & Z409 & RZ409 \\
\hline $\begin{array}{l}\text { Amount of calcined dolomite } \\
\text { (guard bed reactor) }\end{array}$ & $120 \mathrm{ml}(132.16 \mathrm{~g})$ & $120 \mathrm{ml}(132.10 \mathrm{~g})$ & $120 \mathrm{ml}(132.10 \mathrm{~g})$ \\
\hline $\begin{array}{l}\text { Amount of Ni-Based Catalyst } \\
\text { (metal catalyst reactor) }\end{array}$ & $20 \mathrm{ml}(22.30 \mathrm{~g})$ & $20 \mathrm{ml}(23.24 \mathrm{~g})$ & $20 \mathrm{ml}(23.10 \mathrm{~g})$ \\
\hline $\begin{array}{l}\text { Amount of inert material } \\
\text { (metal catalyst reactor) }\end{array}$ & $20 \mathrm{ml}(15.30 \mathrm{~g})$ & $20 \mathrm{ml}(15.20 \mathrm{~g})$ & $20 \mathrm{ml}(15.40 \mathrm{~g})$ \\
\hline Pretreatment of catalyst & No reduction & No reduction & Manufacturer reduced \\
\hline Guard bed temperature, $\mathrm{T}_{\mathrm{GB}}\left( \pm 5^{\circ} \mathrm{C}\right)$ & $650^{\circ} \mathrm{C}$ & $650^{\circ} \mathrm{C}$ & $650^{\circ} \mathrm{C}$ \\
\hline $\begin{array}{l}\text { Catalytic reactor temperature, } \mathrm{T}_{\mathrm{CR}} \\
\left( \pm 3^{\circ} \mathrm{C}\right)\end{array}$ & $740,760,780,800,820^{\circ} \mathrm{C}$ & $740,760,780,800,820^{\circ} \mathrm{C}$ & $740,760,780,800,820^{\circ} \mathrm{C}$ \\
\hline Space Velocity - dry gas basis $\left(\mathrm{h}^{-1}\right)$ & $1500,3000,4500,6000$ & $1500,3000,4500,6000$ & $1500,3000,4500,6000$ \\
\hline $\begin{array}{l}\text { Operating time without steam } \\
\text { injection }\end{array}$ & $\begin{array}{l}12 \mathrm{hrs} \\
(\text { Steam } / \mathrm{C}=2.8)\end{array}$ & $\begin{array}{l}12 \mathrm{hrs} \\
(\mathrm{Steam} / \mathrm{C}=2.8)\end{array}$ & $\begin{array}{l}12 \mathrm{hrs} \\
(\mathrm{Steam} / \mathrm{C}=2.8)\end{array}$ \\
\hline Operating time with steam injection & $0 \mathrm{hrs}$ & $\begin{array}{c}\text { 6hrs } \\
(\text { Steam } / \mathrm{C}=4.5,5.5,6.5)\end{array}$ & $\begin{array}{c}\text { 6hrs } \\
(\text { Steam } / \mathrm{C}=4.5,5.5,6.5)\end{array}$ \\
\hline
\end{tabular}

Table 4 Operating conditions of reactor train when testing water-gas shift catalysts

\begin{tabular}{|c|c|c|c|c|}
\hline Reactor & Guard Bed & $\begin{array}{c}\text { Steam } \\
\text { Reforming }\end{array}$ & $\begin{array}{c}\text { High Temperature } \\
\text { Shift }\end{array}$ & $\begin{array}{c}\text { Low Temperature } \\
\text { Shift }\end{array}$ \\
\hline Reactor set point $\left({ }^{\circ} \mathrm{C}\right)$ & 650 & 800 & 400 & 200 \\
\hline Operating range $\left({ }^{\circ} \mathrm{C}\right)$ & $600-670$ & $750-850$ & $350-420$ & $180-240$ \\
\hline $\mathrm{SV}\left(\mathrm{h}^{-1}\right)$ & 900 & 3000 & 1500 & 1200 \\
\hline Catalyst & Calcined dolomite & ICI 46-1 & Fe-Cr based LB & Cu-Zn-A1 based B202 \\
\hline Catalyst volume $(\mathrm{ml})$ & 200 & 60 & 120 & 150 \\
\hline $\begin{array}{c}\text { Inert material* } \\
(\mathrm{ml} / \mathrm{ml})\end{array}$ & $0 / 20$ & $20 / 20$ & $20 / 50$ & $25 / 50$ \\
\hline
\end{tabular}

\footnotetext{
*Volume of inert material above/below catalyst layer.
} 
at $180^{\circ} \mathrm{C}$. Judging from the hydrogen content exiting the reactors, the Fe-Cr based catalyst was substantially reduced after 20 minutes while the $\mathrm{Cu}-\mathrm{Zn}$ based catalyst required 45 minutes. Reduction was assumed complete when the hydrogen concentration exiting the reactor stopped increasing and reached a steady-state concentration. The catalysts were readily reduced by producer gas without requiring the addition of hydrogen as a reducing agent. Table 4 details operating conditions of the gas conditioning system when evaluating the performance of the water-gas shift catalysts. During trials with the steam reformer and water-gas shift reactors, producer gas was passed through the gas conditioning system at a flow rate of $3.0 \mathrm{~L} / \mathrm{min}$ with steam added to achieve steam/gas volumetric ratio of 1.2. The guard bed was operated at a temperature $\mathrm{T}_{\mathrm{GB}}$ equal to about $650^{\circ} \mathrm{C}$ and space velocity of $900 \mathrm{~h}^{-1}$ while the steam reformer (tar reactor) was operated at a temperature $\mathrm{T}_{\mathrm{TR}}$ equal to about $800^{\circ} \mathrm{C}$ and space velocity of $3000 \mathrm{~h}^{-1}$. Gas composition was measured after each of the three catalytic reactors and $\mathrm{CO}$ conversion calculated for each of the water-gas shift reactors.

Gas composition both upstream and downstream of the gas conditioning system was periodically analyzed by on-line gas chromatography using a Varian Micro-GC CP-2003 Quad equipped with Molsieve 5A BF, Poraplot Q, and CP-Sils CB columns and a thermal conductivity detector with argon as carrier gas for the first column and helium as carrier gas for the second and third columns. The first column gave $\mathrm{H}_{2}, \mathrm{O}_{2}, \mathrm{~N}_{2}, \mathrm{CH}_{4}$, and $\mathrm{CO}$ concentrations; the second and third columns yielded $\mathrm{CO}_{2}, \mathrm{C}_{2} \mathrm{H}_{4}$ and some light hydrocarbons. Continuous monitoring of exit gases was achieved with non-dispersive infrared analyzers for $\mathrm{CO}$ and $\mathrm{CO}_{2}$, a Nova thermal conductivity analyzer for $\mathrm{H}_{2}$, and a California Analytic electrochemical sensor for $\mathrm{O}_{2}$. The reliability of the analyzers was checked periodically using calibration gas. The volumetric flow rate of the dry gas exiting the gas conditioning system was measured by means of a wet test meter and reported at normal conditions.

Analysis of the extent of conversion of $\mathrm{CO}$ by the water gas shift reaction requires careful consideration of changes in mole fractions of the reacting gases. Although the water gas shift reaction is an equal molar reaction, resulting in no net volume change, one must account for the fact that gas analysis is performed on a dry gas basis. Let $\chi$ be defined as the molar conversion of $\mathrm{CO}$ :

Eq. 9

$$
\chi=\frac{n_{C O_{\text {inlet }}}-n_{C O_{\text {outlet }}}}{n_{C O_{\text {inlet }}}}
$$

where $\mathrm{n}_{\mathrm{CO}}$ designates the number of moles of $\mathrm{CO}$ at the inlet or outlet of the reactor as appropriate. Since the water-gas shift reaction is equimolar, the total number of moles at the inlet and outlet of the reactor are equal and it follows that:

Eq. 10

$$
\chi=\frac{X_{C O_{\text {inlet }}}-X_{C O_{\text {outlet }}}}{X_{C O_{\text {inlet }}}}
$$


where $\mathrm{X}$ designates mole fractions based on water vapor being one of the constituents of the gas mixture. In practice, gas analysis is performed on dry gas, the water vapor having been removed from the gas mixture before analysis; thus, it is convenient to define mole fractions that are based on moles of dry gas:

Eq. $11 \quad X_{C O}^{\prime} \equiv \frac{n_{C O}}{n_{D G}}$

where $\mathrm{n}_{\mathrm{DG}}$ is the total moles of dry gas. Solving Eq. 11 for $\mathrm{n}_{\mathrm{CO}}$, substituting this expression into Eq. 9, and recognizing that the change in moles of dry gas through the reactor is equal to the change in moles of $\mathrm{CO}$ through the reactor, it can be shown that the molar conversion of $\mathrm{CO}$ expressed in terms of $\mathrm{X}^{\prime}{ }_{\mathrm{CO}}$ is:

Eq. 12

$$
\chi=\frac{X_{C O_{\text {inlet }}}^{\prime}-X_{C O_{\text {outlet }}}^{\prime}}{X_{C O_{\text {inlet }}}^{\prime}\left(1+X_{C O_{\text {outlet }}}^{\prime}\right)}
$$

Upon completion of the trials, both fresh and spent catalysts were characterized by BET specific surface and porosity and by X-ray photoelectron spectroscopy (XPS). Analysis by XPS was performed to check for coking and poisoning of the catalysts by chlorine and sulfur. Specific surface area and porosity were tested using ASAP 2010 with analysis adsorptive N2 at 77.35K. Analysis by XPS was performed using a Physical Electronics 5500 Multitechnique system with monochromatic $\mathrm{Al}$ and standard $\mathrm{Mg} / \mathrm{Al}$ sources using sample sizes of less than $2 \mathrm{~cm} \times 2 \mathrm{~cm}$. Although analysis of reduced catalyst would have been useful in understanding the development of pore structure, it was not possible with the present experimental facilities to remove a reduced catalyst sample from the reactors without it being exposed to the oxidizing environment of the atmosphere. Thus, only fresh and spent catalysts were analyzed.

\section{Combined Catalyst/Sorbent System}

In addition to exploring the performance of conventional catalytic systems for promoting the water gas shift reaction, work was performed to demonstrate a combined catalyst/sorbent system that would simultaneously perform the water gas shift reaction and absorb the carbon dioxide released by this reaction. Such a material can be used in a single fixed or moving bed system to integrate the functions of chemical reaction and product separation. The advantages of this approach include increasing the maximum possible equilibrium conversion to hydrogen as well as separating the carbon dioxide from the hydrogen. The absorbed carbon dioxide could be subsequently processed for sequestration. Increasing the equilibrium conversion of the water gas shift reaction provides the potential for obviating the need for the two-step reaction scheme currently used in a water gas shift process where a high temperature shift reactor is followed by a low temperature shift reactor.

The two stages included formation of a spherical limestone core followed by coating with a mixture of alumina and limestone particles. High temperature treatment converts the core material to $\mathrm{CaO}$ and partially sinters the shell material. The resulting core-in-shell pellets have highly reactive cores encased in porous but strong shells that are essentially inert. Besides using 
limestone and dolime as sorbent core materials, pellets were also made using new sorbent materials called Kemidol Hydrate $(\mathrm{Ca}(\mathrm{OH}) 2 \mathrm{MgO})$ and Kemidol Superhydrate $\left(\mathrm{CaMg}(\mathrm{OH})_{4}\right)$, which were produced by Graymont Dolime $(\mathrm{OH})$ Inc.

The catalyst/sorbent pellets were tested in the fixed bed reactor system illustrated in Figure 12. A core-in-shell sorbent (not treated with nickel impregnation) was tested for the water-gas shift reaction. It was found that the nickel catalyst was required for the water-gas-shift reaction at reaction temperature lower than $600^{\circ} \mathrm{C}$. At temperature higher than $600^{\circ} \mathrm{C}$, the same levels of carbon monoxide conversions were obtained from reactions using pellets regardless of whether or not the pellets were loaded with nickel. The performance of pellets made of Kemidol Hydrate and Kemidol Superhydrate cores was also tested for adsorbing $\mathrm{CO}_{2}$. The $\mathrm{CO}_{2}$ absorption capacity of the new sorbent materials was found to be higher than that of dolimite. Calcining the materials at different temperatures, the $\mathrm{CO}_{2}$ absorption capacity of the materials was found to be lower after the pellets were calcined at $1100^{\circ} \mathrm{C}$.

Core-in-shell pellets were prepared in which the shell material was loaded with ferric oxide at various concentrations to be used in the water-gas-shift reaction. The pellets were found to be structurally very strong. Calcination at $900^{\circ} \mathrm{C}$ resulted in pellets having average crush strengths of not less than $9 \mathrm{~N} / \mathrm{mm}$ pellet diameter. With the standard alumina-limestone mixture shell material, this result could only be obtained if the pellets had undergone calcination at $1100^{\circ} \mathrm{C}$. The pellets were tested for the water-gas-shift reactions in the fixed bed reactor.

\subsection{Gas Sampling System}

\section{Trace Contaminant System}

In our system, trace contaminant gases such as $\mathrm{NH}_{3}$ and $\mathrm{H}_{2} \mathrm{~S}$ are collected at relatively low temperatures (i.e., less than $110^{\circ} \mathrm{C}$ ). Therefore, the sampling system must either remove the heavy tars without losing the gas species of interest, or else tar condensation must be avoided all together. Losses of $\mathrm{NH}_{3}$ and $\mathrm{H}_{2} \mathrm{~S}$ resulting from interactions with components of the sample transport system (e.g., sorption onto the walls of the sampling lines) must also be avoided. In addition, since $\mathrm{NH}_{3}$ and $\mathrm{H}_{2} \mathrm{~S}$ are water soluble, condensation of moisture during sample transport can not be allowed to occur in the sample lines. If moisture condensation occurs, a portion of the $\mathrm{NH}_{3}$ and $\mathrm{H}_{2} \mathrm{~S}$ will dissolve in the moisture and constitute a loss in some of those gas species. With those considerations in mind, a sampling system was designed and built for sampling trace contaminant gases such as $\mathrm{NH}_{3}$ and $\mathrm{H}_{2} \mathrm{~S}$ in the syngas stream. A schematic diagram of the trace contaminant sampling system installed on the gasifier is shown in Figure 13. The system is set up for sampling $\mathrm{NH}_{3}$ using impingers, while direct gas analyses are used for the sulfurcontaining species (i.e., $\mathrm{H}_{2} \mathrm{~S}$ and $\mathrm{COS}$ ).

Syngas is drawn through the sampling system at a flow rate of about $2 \mathrm{~L} / \mathrm{min}$ (STP). All sample lines upstream from the tar condenser are maintained at a temperature of $450^{\circ} \mathrm{C}$ in order to prevent tar condensation. Particulate matter is removed from the gas stream by using a small ( 25 x $90 \mathrm{~mm}$ ) quartz-fiber thimble filter in a custom-built steel assembly. The filter assembly is housed in an oven maintained at $450^{\circ} \mathrm{C}$. After removing particulate matter from the gas stream, the sample gases enter a tar condensation coil. The condensation coil consists of 10-20 feet of 


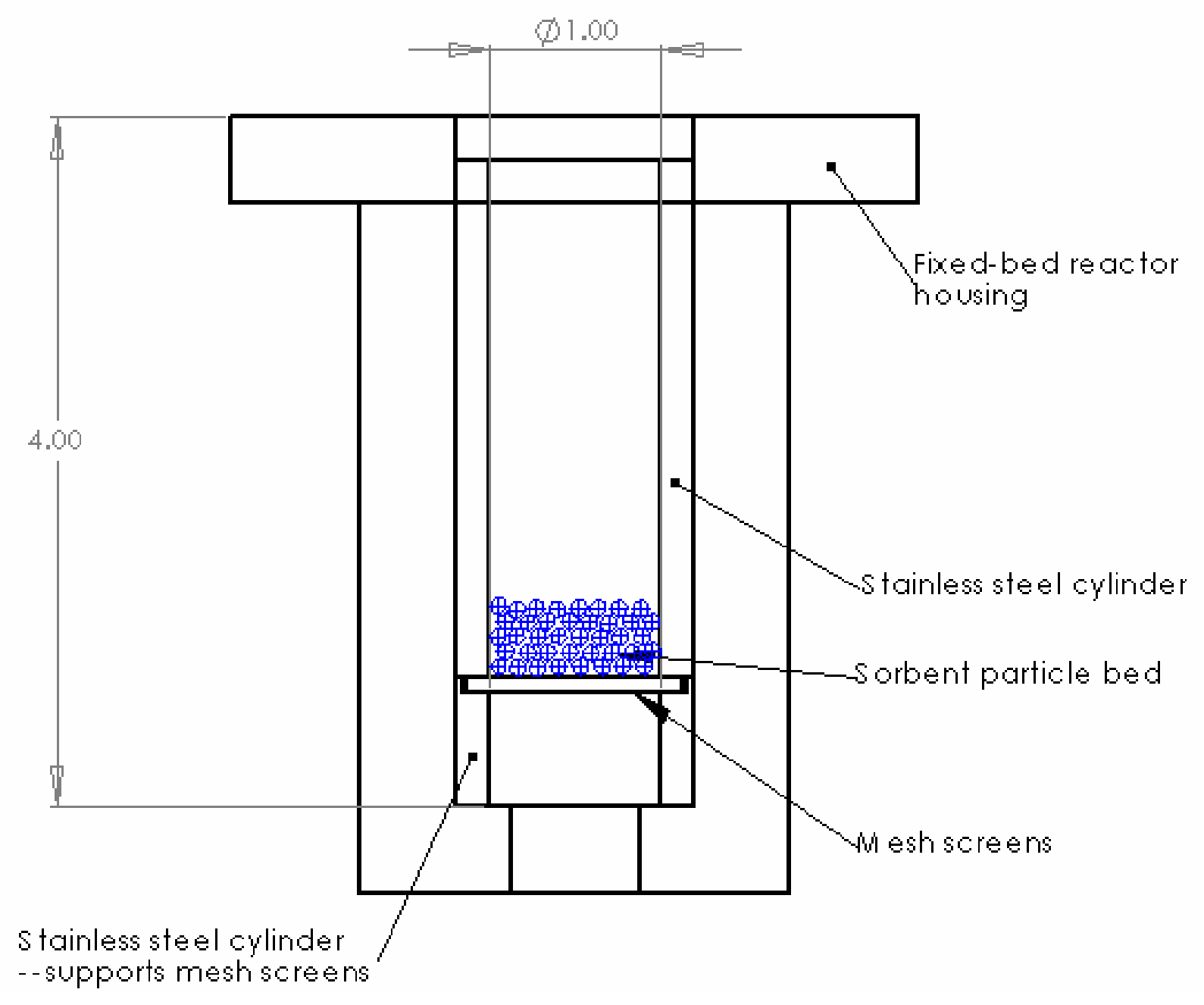

Figure 12 Fixed bed reactor employed in evaluations of combined catalyst/sorbent system for water-gas shift reaction 


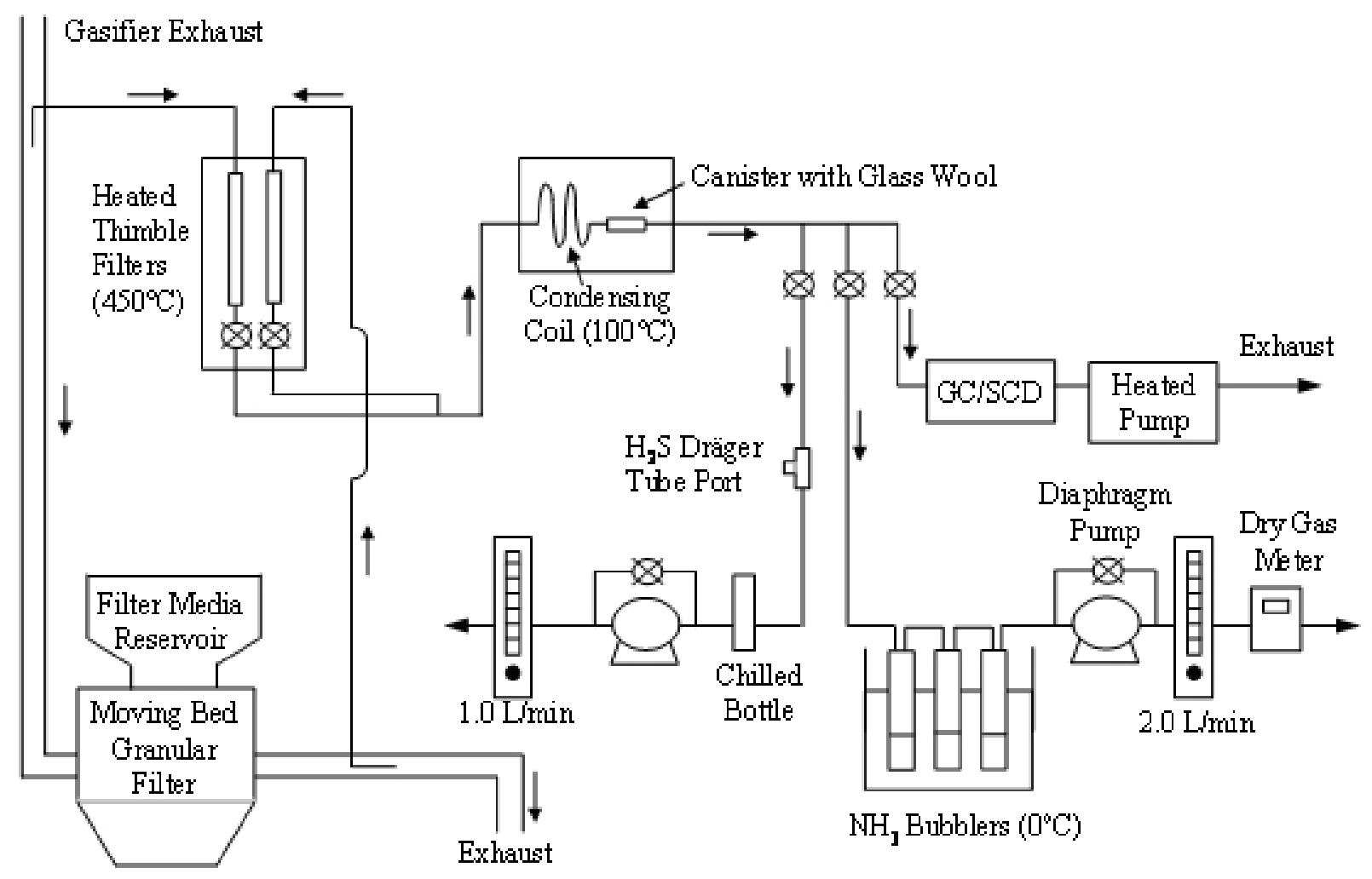

Figure 13 Schematic diagram of trace contaminant sampling system 
$3 / 8$ " $(9.5 \mathrm{~mm})$ OD tubing that is housed in a modified, home-style pressure cooker that is partially filled with water and heated to about $102^{\circ} \mathrm{C}$, which is above the moisture dew point of the gas stream. When the gases enter the tar condenser, the gas temperature quickly drops to about $102^{\circ} \mathrm{C}$. This allows the heavy tars to condense in the coil while avoiding moisture condensation. Light hydrocarbons remain in the gas stream at this point. The inner diameter of the tubing is sufficiently large to avoid plugging the coil with tar during sampling. Because tar aerosols are not always effectively removed by the coil, a canister containing glass wool is also housed in the pressure cooker and is attached to the exit of the condensation coil. This canister effectively removes the majority of heavy tar aerosols from the gas stream. The sample lines downstream from the tar condenser are heated to about $110^{\circ} \mathrm{C}$ to ensure that no light hydrocarbons or moisture condense.

Sampling for $\mathrm{NH}_{3}$ is performed by bubbling syngas through a series of impingers containing 5\% (v/v) $\mathrm{HCl}$ or $\mathrm{H}_{2} \mathrm{SO}_{4}$. Sampling is generally performed for 15-30 minutes. The impingers are maintained at about $0^{\circ} \mathrm{C}$ by keeping them in an ice bath during sampling. After sampling, there is typically a colloidal suspension in the impingers. Therefore, the impinger solutions are filtered within 30 minutes of sample collection. Additional details on $\mathrm{NH}_{3}$ sampling procedures and on processing of the impinger solutions prior to analysis are presented elsewhere. ${ }^{15}$

\section{Sample Transport Studies in the Laboratory}

Maintaining sample integrity during sample transport is a critical prerequisite of the overall methodology for determining low levels of $\mathrm{NH}_{3}$ and $\mathrm{H}_{2} \mathrm{~S}$. Therefore, numerous tests were performed to assess sample transport issues for the trace contaminant gases of interest. Laboratory tests showed that $\mathrm{H}_{2} \mathrm{~S}$ could be passed effectively through Teflon lines at room temperature, even for $\mathrm{H}_{2} \mathrm{~S}$ concentrations in the 0-5000 ppb range. Laboratory tests also showed that a stream of $25 \mathrm{ppm}_{2} \mathrm{~S}$ (in nitrogen) could be successfully transported through stainless steel (type 316) lines at 25 and $200^{\circ} \mathrm{C}$, but major $\mathrm{H}_{2} \mathrm{~S}$ losses were observed when the steel was at $400^{\circ} \mathrm{C}$. Using electropolished stainless steel or superalloy Inco Alloy C276 did not help avoid $\mathrm{H}_{2} \mathrm{~S}$ losses at that temperature. However, stainless steel with an interior coating of amorphous silica effectively transported low levels of $\mathrm{H}_{2} \mathrm{~S}$ at $400^{\circ} \mathrm{C}$. Therefore, all sample transport lines and other sampling components in our sampling system that are heated above $200^{\circ} \mathrm{C}$ are made of silica-coated steel. Below that temperature, Teflon lines are used to help ensure effective sample transport over a wider range of $\mathrm{H}_{2} \mathrm{~S}$ concentrations. Laboratory tests with $60 \mathrm{ppm} \mathrm{NH}_{3}$ indicated that this gas could be effectively transported through uncoated stainless steel lines at $25-400^{\circ} \mathrm{C}$. Therefore, the primary reason for using the silica-coated steel lines in our sampling system is to aid in the transport of $\mathrm{H}_{2} \mathrm{~S}$.

Since efforts are underway to develop our capability to sample and measure $\mathrm{HCl}$, tests were also performed to study the transport of $\mathrm{HCl}$ through various types of sample lines. Initially, laboratory tests with $50 \mathrm{ppm} \mathrm{HCl}$ in nitrogen were performed. That gas stream was passed through sample lines made of a variety of materials, including Teflon, 316 stainless steel, electropolished stainless steel, silica-coated steel, and superalloy Inco Alloy C276. Sample transport in the 316 stainless steel was poor at room temperature, and was somewhat better with the Inco Alloy $\mathrm{C} 276$. However, the best $\mathrm{HCl}$ transport at room temperature was obtained using silica-coated steel, Teflon, and electropolished stainless steel. At temperatures of 200 and 
$400^{\circ} \mathrm{C}$, the $\mathrm{HCl}$ was effectively transported in all the metal lines tested. Based on these results, sample lines below about $200^{\circ} \mathrm{C}$ should be made of either Teflon or silica-coated steel.

\section{Sample Transport through the Sampling System}

Prior to the installation of a quartz thimble filter for ash removal in syngas streams, a large, highly porous, sintered metal filter had been used for ash filtration. While using the sintered steel filter, $1000 \mathrm{ppm} \mathrm{NH}_{3}$ (in nitrogen) could be effectively transported through the heated system with negligible losses. However, $100 \mathrm{ppm}_{2} \mathrm{~S}$ (in nitrogen) was not effectively transported through that filter. In fact, virtually none of the $\mathrm{H}_{2} \mathrm{~S}$ was getting transported downstream, even after the gases had been flowing for up to an hour. Tests showed that the filter was the source of the $\mathrm{H}_{2} \mathrm{~S}$ losses. This necessitated that modifications be made in the gas filtration system. Instead of using a large, highly porous metal filter, a new filtering system was designed and tested. The new filter used a disposable quartz-fiber thimble filter in a silica-coated steel housing.

After replacing the sintered metal filter with the quartz thimble filter, the transport of $\mathrm{H}_{2} \mathrm{~S}$ and $\mathrm{NH}_{3}$ was studied using a gas blend containing $500 \mathrm{ppm} \mathrm{NH} 3$ and $50 \mathrm{ppm}_{2} \mathrm{~S}$ in nitrogen. Drager tubes were used to determine concentrations of $\mathrm{NH}_{3}$ and $\mathrm{H}_{2} \mathrm{~S}$ downstream from the tar condenser. The thimble filter and the tar condensation coil were both "dirty" (with ash and tar, respectively) from a previous gasification test in order to more closely simulate actual sampling conditions. Test results indicated that the $\mathrm{NH}_{3}$ once again passed through the entire heated sampling system quickly, reaching its full concentration of $500 \mathrm{ppm}$ downstream from the tar condenser within about 5 minutes. With the dirty filter and tar condensation coil, the $\mathrm{H}_{2} \mathrm{~S}$ was transported less efficiently than $\mathrm{NH}_{3}$, but was much more efficient with the quartz thimble filter than with the sintered steel filter. The ash in the filter was found to be an issue in the transport of $\mathrm{H}_{2} \mathrm{~S}$. When a heavily loaded ash filter was in place, it took over 15 minutes for the $\mathrm{H}_{2} \mathrm{~S}$ at the sampling port to reach its full concentration of $50 \mathrm{ppm}$. However, when a clean quartz thimble filter was used, the $\mathrm{H}_{2} \mathrm{~S}$ was effectively transported through the entire heated sampling system, reaching full $\mathrm{H}_{2} \mathrm{~S}$ concentrations (i.e., $50 \mathrm{ppm}$ ) downstream from the tar condenser within 5 minutes. The sintered metal filter was permanently replaced by the quartz thimble filter in the sampling system since it is easier and less costly to maintain, dramatically decreases the surface area of the filter (thereby minimizing the potential for analyte losses on the wall surfaces), and is nonreactive compared to steel. The later point is an issue primarily for $\mathrm{H}_{2} \mathrm{~S}$.

Several tests were also performed to assess $\mathrm{HCl}$ transport through the sampling system on the gasifier. Blended gas streams containing known concentrations of $\mathrm{HCl}, \mathrm{NH}_{3}$, and water in a nitrogen balance were used. Those blended gases were passed through a quartz thimble filter loaded with char and ash, and through a tar condensation coil loaded with tar from a previous gasification trial. The intent was to determine whether accumulated particulate matter and tar would interfere with $\mathrm{HCl}$ sampling. Base on those tests, it was concluded that $\mathrm{HCl}$ transports very well in the existing trace contaminant sampling system in the presence of particulate and tar. However, once $\mathrm{NH}_{3}$ is included in the gas blend, it reacts with $\mathrm{HCl}$ at low temperatures to produce ammonium chloride, which precipitates in the sampling line. We are currently developing an approach that maintains a gas temperature that is high enough to prevent the formation of solid ammonium chloride until $\mathrm{HCl}$ can be collected in impingers containing $2.5 \%$ $\mathrm{NaOH}$, which collects both $\mathrm{HCl}$ and $\mathrm{H}_{2} \mathrm{~S}$. 


\subsection{Gas and Trace Contaminant Analysis}

\section{Analysis of Major Gas Constituents}

Gas samples were analyzed on-line by gas chromatography using a Varian Micro-GC CP-2003 Quad equipped with Molsieve 5A BF, Poraplot Q, and CP-Sil CB columns and a thermal conductivity detector. Argon was used as the carrier gas for the first column and helium as the carrier gas for the second and third columns. The Molsieve $5 \mathrm{~A}$ BF column provided $\mathrm{H}_{2}, \mathrm{O}_{2}, \mathrm{~N}_{2}$, $\mathrm{CH}_{4}$, and $\mathrm{CO}$ concentrations; the Poraplot $\mathrm{Q}$ and $\mathrm{CP}-\mathrm{Sil} \mathrm{CB}$ columns yielded $\mathrm{CO}_{2}, \mathrm{C}_{2} \mathrm{H}_{4}$, and some light hydrocarbons. The micro-GC is capable of processing one sample every three minutes. Gas analysis is also performed using a Nova Model 430RM hydrogen analyzer and a California Analytic Model 300 Infrared Carbon Dioxide/Carbon Monoxide/Oxygen analyzer operating in a parallel configuration. Prior to analysis in any instrument, the gas is conditioned by removing all particulate, tar, and moisture. Concentrations are reported on a dry, volume/volume basis.

\section{$\underline{\text { Sulfur Measurements }}$}

For measuring $\mathrm{H}_{2} \mathrm{~S}$, a Zellweger Analytics Model 7100 Toxic Gas Monitor was considered for on-line $\mathrm{H}_{2} \mathrm{~S}$ analyses. This method is based on passing sample gas through chemicallyimpregnated tapes. The presence of $\mathrm{H}_{2} \mathrm{~S}$ in the gas stream causes a color change on the tape, which is then measured by monitoring the reflectivity of the tape. The change in reflectivity is related to the $\mathrm{H}_{2} \mathrm{~S}$ concentration. Laboratory tests with the instrument indicated that the linearity, precision, and sensitivity were excellent under the proper conditions. Also, there are no significant interferences for the gas streams of interest. However, in order to obtain accurate results, the relative humidity $(\mathrm{RH})$ of the gas stream must be constant and must be within $10 \%$ $\mathrm{RH}$ (absolute) of the optimum $\mathrm{RH}$ value, which is in itself sensitive to the $\mathrm{H}_{2} \mathrm{~S}$ concentration and a variety of instrumental variables. Because of the high sensitivity to $\mathrm{RH}$, plus the fact that the dynamic range of the instrument in the ppm range is very narrow (e.g., 0-50 ppm), the overall utility of this method is considered marginal for determining $\mathrm{H}_{2} \mathrm{~S}$ in the application of interest.

Drager tubes were also investigated for measuring $\mathrm{H}_{2} \mathrm{~S}$ concentrations. The tubes are not sensitive to relative humidity (a major advantage over the Zellweger analyzer) and the tube readings are very linear with $\mathrm{H}_{2} \mathrm{~S}$ concentration. In addition, for the sample streams of interest, there do not appear to be any significant interference. Organic vapors in the sample gas have little or no effect on the $\mathrm{H}_{2} \mathrm{~S}$ reading, and the organic vapors themselves do not give a reading. The readings from the Drager tube are expected to be accurate to within 25-30\%. However, the precision is very good, which makes this approach well suited to tracking relative $\mathrm{H}_{2} \mathrm{~S}$ concentrations if good sampling techniques are utilized. As such, these tubes have provided a simple and convenient method for determining the fate of $\mathrm{H}_{2} \mathrm{~S}$ during sorbent testing experiments. The Drager tubes appear to work well, and are attractive because of their low cost (about $\$ 5$ per test), simplicity, and speed (less than 1 minute). Therefore, they are used routinely for spot checks on $\mathrm{H}_{2} \mathrm{~S}$ concentrations and in other monitoring applications where high accuracy is not needed.

Although Drager tubes have proven to be suitable for monitoring $\mathrm{H}_{2} \mathrm{~S}$ concentrations in most cases, they do not have a high degree of accuracy, do not provide a continuous monitoring capability, and do not provide simultaneous results for different sulfur-containing gas species. 
Therefore, our trace contaminant system is also equipped with a gas chromatograph (GC-17A, Shimadzu Co. Ltd.) combined with a sulfur chemiluminescence detector (355 SCD, Ionics Instrument Business Group) for continuous measurement of sulfur species (mostly $\mathrm{H}_{2} \mathrm{~S}$ and COS). The integrated GC-SCD is extremely sensitive (detection limit in the low ppb range), highly specific, have a large linear range (over five orders of magnitude), and are not adversely affected by the hydrocarbons in the gas stream. In addition, responses to different sulfur compounds with the SCD are equimolar, meaning that a given concentration of a sulfur compound gives the same response, regardless of the specific sulfur compound being determined. This provides the advantage of being able to calibrate for all sulfur species with a single sulfur compound. Numerous columns are available for separating sulfur species, and each has their own advantages and disadvantages. Columns were screened to help determine which ones are best suited for our application. Factors such as temperature limitations, elution order for different sulfur species (important to avoid interferences), and degree of separation between different sulfur forms were considered. This screening procedure resulted in the selection of two capillary columns that seem best suited for our work.

\section{$\underline{\text { Ammonia Measurements }}$}

The Zellweger analyzer (discussed above) and Drager tubes were investigated as potential methods for determining $\mathrm{NH}_{3}$ in syngas streams. However, severe chemical interferences and/or high sensitivity to the RH of the gas stream make those approaches unsuitable for the analyses of interest. Subsequent efforts focused on the use of off-line wet chemical approaches for determining $\mathrm{NH}_{3}$ in the syngas streams.

A fluorometric method for the determination of $\mathrm{NH}_{4}\left(\mathrm{NH}_{3}\right.$ in water produces $\left.\mathrm{NH}_{4}\right)$ in aqueous solutions was investigated as a fast, inexpensive, and simple analytical approach for in-house wet chemical determinations of $\mathrm{NH}_{3}$. The fluorometric method is desirable because the instrument is very small (about $0.5 \mathrm{ft}^{3}$ ), requires little maintenance, and is very easy to operate. The method is based on reacting $\mathrm{NH}_{4}$ with orthophthaldialdehyde to form a highly fluorescent compound, which is then measured with a fluorometer. The approach was studied in the laboratory and was found to be extremely sensitive, with a detection limit of less than $1 \mathrm{ppb}$ in solution. Under the conditions studied, the linear range was from about $0-200 \mathrm{ppb}$ in solution. Based on the analyses of actual field samples (aqueous condensates) obtained during biomass gasification, autofluorescence (fluorescence of the matrix itself) was not a problem. However, when solutions had $\mathrm{NH}_{4}$ concentrations typical of those found in impinger solutions from field sampling operations (i.e, several hundred $\mu \mathrm{g} \mathrm{NH} / \mathrm{mL}$ of solution), the solutions became very cloudy and accurate analysis of the solutions could not be performed. Even the analytical standards became

very cloudy when $\mathrm{NH}_{4}$ concentrations exceeded about $1 \mu \mathrm{g} / \mathrm{mL}$ in solution. Attempts were made to modify the fluorometric procedure in order to eliminate the cloudiness in the concentration range of interest, and also to extend the linear range. This included modifying 6 to 10 analytical variables. However, short of diluting the sample solutions into the range of $0-200 \mathrm{ng} \mathrm{NH} / \mathrm{mL}$, none of the approaches were successful in avoiding the cloudy solutions. Performing a 10,000:1 sample dilution is very easy and might allow the fluorometric method to be used for fast and easy on-site analyses of impinger solutions from field sampling operations. However, rather than performing on-site determinations, it was decided to send samples of impinger solutions to a nearby analytical laboratory that provides rapid and inexpensive analyses of those solutions. 
Impinger solutions used to sample for $\mathrm{NH}_{3}$ were initially analyzed by ion chromatography (IC). However, results of those analyses indicated that there was little or no $\mathrm{NH}_{3}$ present in the gas stream, even though there should have been a substantial amount of $\mathrm{NH}_{3}$ present. A series of tests were performed in the laboratory to help determine the source of the error. In one series of tests, a variety of liquid $\mathrm{NH}_{4}$ standards in $5 \% \mathrm{H}_{2} \mathrm{SO}_{4}$ were prepared and submitted for IC analyses. No $\mathrm{NH}_{4}$ could be detected in any sample, even though all of the concentrations used should have been easily detectable. This indicated a significant analytical problem for this particular sample matrix. The standards were sent to another analytical laboratory with IC capabilities, and they were also unable to detect any $\mathrm{NH}_{4}$. Attempts were made to neutralize the sample (e.g., with $\mathrm{NaOH}$ ), but cations associated with the neutralizing agents caused interference problems. Since a conductivity detector is used for the analysis, the analysts believe the analytical problem is due to excessive ions in solution (from the acid), which in turn leads to a large ionic background that makes $\mathrm{NH}_{4}$ detection very difficult at low levels.

Next, a series of $10 \mathrm{ppm}_{\mathrm{w}} \mathrm{NH}_{4}$ standards in $\mathrm{H}_{2} \mathrm{SO}_{4}$ solutions of varying acid concentration were submitted for IC analyses in order to determine the effects of acid strength on the IC results. For each acid strength, two sets of identical $10 \mathrm{ppm}_{\mathrm{w}}$ standards were prepared and submitted.

Results of those tests are shown in Table 5. As can be seen from the data, the acid strength had a strong effect on the analytical results. As noted above, the high ionic background associated with the concentrations of $\mathrm{H}_{2} \mathrm{SO}_{4}$ used for the sample matrix is probably responsible for the analytical difficulties.

Next, $50 \mathrm{ppm}_{\mathrm{w}} \mathrm{NH}_{4}$ standards in $0.02,0.5$, and 5.0\% $\mathrm{H}_{2} \mathrm{SO}_{4}$ were submitted to three different laboratories for analysis. One laboratory performed the analysis by colorimetry, one by titration, and one by ion selective electrode (ISE). Results of those analyses indicated that excellent results were obtained using both the colorimetric and titrimetric analyses, but not for the analyses by the ISE method. For that method, it is anticipated that the high ionic strength (due to $\mathrm{H}_{2} \mathrm{SO}_{4}$ ) of the solutions was causing errors in the analysis, as was probably the case for the analyses by IC. Because of the excellent titrimetric results, and because an analytical laboratory using that procedure was in close proximity to the biomass gasifier (thereby facilitating sample submissions), titration was selected as the analytical method to be used for performing routine analyses on the impinger solutions. Results of those titrimetric analyses are subsequently used to calculate $\mathrm{NH}_{3}$ concentrations in the syngas from the biomass gasifier. This is done by dividing the total amount (in $\mu \mathrm{g}$ ) of $\mathrm{NH}_{3}$ collected by the total volume of dry gas (at STP) sampled.

\section{$\underline{\mathrm{HCl} \text { Measurements }}$}

As with the other trace contaminants of interest, the Zellweger analyzer and Drager tubes were investigated to determine if they were suitable for our application of interest. Because of extreme sensitivity to $\mathrm{RH}$ and a very narrow dynamic range, the Zellweger analyzer was found to be unsuitable for measuring $\mathrm{HCl}$ concentrations in the syngas. The Drager tubes worked well overall. The tube readings track well with changing $\mathrm{HCl}$ concentration, and there do not appear to be any significant interferences for our application. However, errors associated with RH and vapor-phase organics preclude their use for accurate $\mathrm{HCl}$ measurements in gasifier streams. 
We are currently developing a wet-chemical sampling procedure that employs analysis of basic impinger solutions with a Hewlett-Packard 4500 Series ICP-MS. That analytical method can be used to measure both $\mathrm{Cl}^{-}$and $\mathrm{S}^{2-}$ ions in the impinger solutions.

Table 5 Effect of acid strength on IC results obtained on $10 \mathrm{ppm}_{\mathrm{w}} \mathrm{NH}_{4}$ standards

\begin{tabular}{|c|c|c|}
\hline \multirow[b]{2}{*}{ Acid Strength of Solution (\%) } & \multicolumn{2}{|c|}{ Reported $\mathrm{NH}_{4}{ }_{4}$ Concentration $\left(\mathrm{ppm}_{\underline{\mathrm{w}}}\right)$} \\
\hline & Sample Set 1 & Sample Set 2 \\
\hline 5 & 0 & 0 \\
\hline 2 & 3.6 & 0 \\
\hline 1 & 1.0 & 0.8 \\
\hline 0.5 & 8.6 & 2.2 \\
\hline 0.2 & 7.4 & 3.0 \\
\hline 0.1 & 4.4 & 1.5 \\
\hline
\end{tabular}

\subsection{Isokinetic sample system}

Isokinetic sampling was employed to establish the filtration efficiency of the moving bed granular filter (MBGF). A modified EPA method 5 for one-point isokinetic sampling is employed to determine particulate matter concentrations in the producer gas stream. The standard EPA method $5^{16}$ is intended for sampling combustion gases, whereas we are sampling from hot producer gas. A one-point method was employed due to the difficulty of traversing a duct that contains hot, tar-laden producer gas under a slight pressure. Sampling ports are positioned the appropriate number of duct diameters before and after bends and valves, as prescribed by Method 5 (see Figure 14). However, we cannot dismiss the possibility that velocity or concentration gradients exist in the flow, which would confound the measurements.

An S-type pitot tube was used to establish the velocity profile and identify a point of average velocity at the particulate sample location. An S-type pitot tube is located at a downstream location, also at a point of average velocity, to measure an instantaneous gas velocity during sampling. This instantateous gas velocity is used to determine an appropriate rate for the isokinetic sample lines.

The sample nozzle, samples lines, and particulate collection filter are all heated to $450^{\circ} \mathrm{C}$ to prevent condensation of tar and moisture. An EPA specified sample nozzle with a $3 \mathrm{~mm}$ diameter was employed. The sample nozzle was fixed at a point of average velocity within the duct. A $9.5 \mathrm{~mm}$ line was used to transport the sample from the nozzle to the particulate filter. A quartz fiber thimble filter is enclosed in a stainless steel filter holder, which is mounted in a heated enclosure. After removal of particulate in by the filter, the producer gas is cooled in an impinger train to remove tars and moisture. A vacuum pump is used to draw the sample gases through the sample nozzle, transport line, filter, and impinger before discharging them to a 


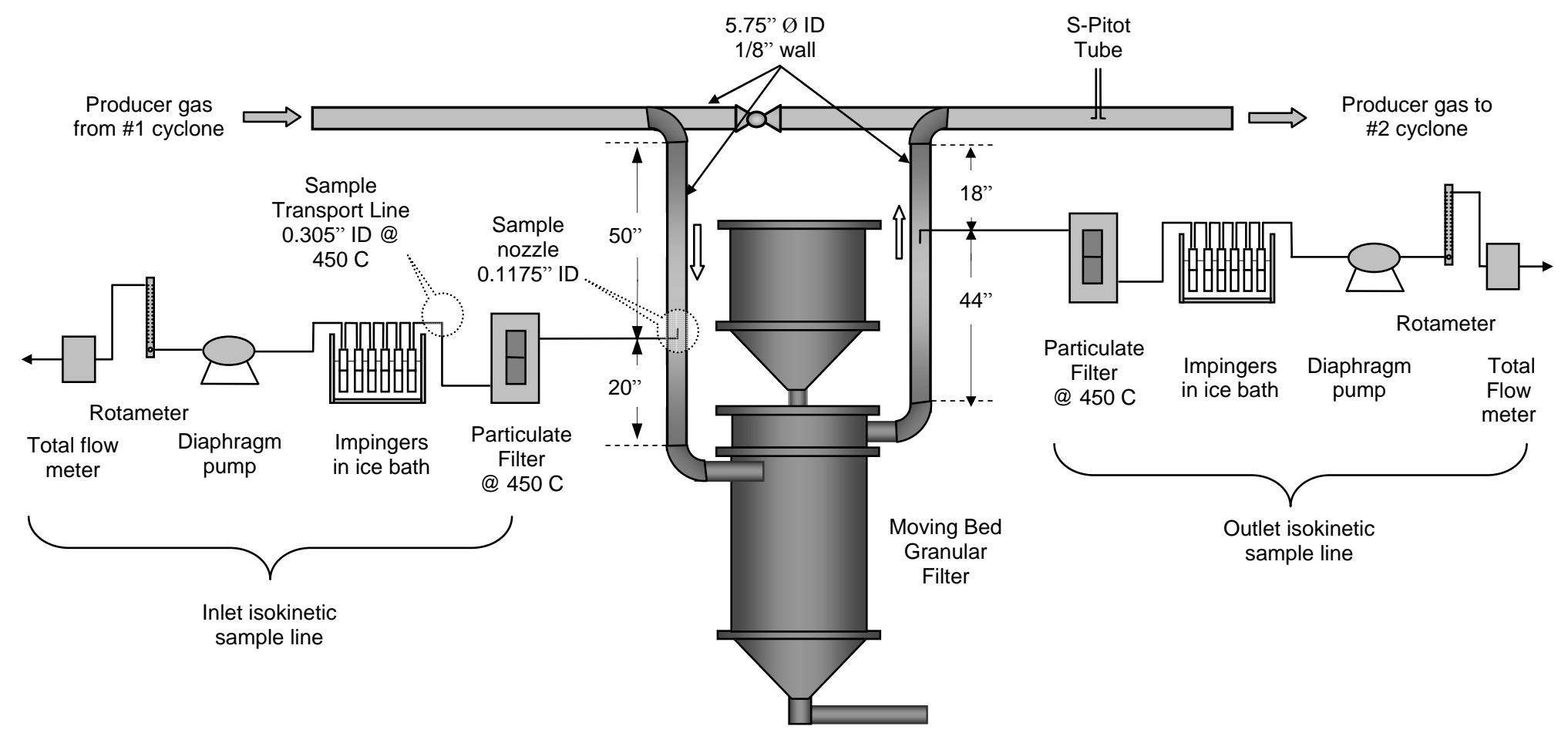

Figure 14 Schematic of isokinetic sample line 
rotameter and a total volume meter. The rotameter is used to control the sample gas flow rate. The total volume meter records the total volume of producer gas sampled for a given experiment.

Particulate concentrations at the inlet and outlet of the MBGF, $\mathrm{C}_{\text {inlet }}$ and $\mathrm{C}_{\text {exit }}$, respectively, were calculated by dividing the weight change in the appropriate filter by the total gas flow through the sampling line. The particulate collection efficiency, $\eta$, of the MBGF was determined from the relationship:

Eq. $13 \quad \eta=1-\frac{C_{\text {exit }}}{C_{\text {inlet }}}$

\subsection{Sorbent Testing System}

A fixed bed reactor (FBR) was fabricated to evaluate the effectiveness of different materials to absorb hydrogen sulfide from a representative slip-stream of producer gas. This custom designed reactor uses a comparable design as the thimble filters used for particulate removal. However, instead of mounting a quart thimble filter in the housing, the housing was modified to support sorbent on a metal screen and provides proper gas flow.

Figure 15 illustrates how two stainless steel cylinders were added to the stainless steel housing to create the FBR. A stainless steel wire mesh with $1.16 \mathrm{~mm}(0.0455 \mathrm{in}$.) openings was sandwiched between the two cylinders to provide support for the bed of sorbent particles. A K-type thermocouple was installed to measure the sorbent bed temperature. A non-reactive, hightemperature caulk was applied to the top-most cylinder to ensure no syngas bypassed the sorbent bed. All reactive surfaces were treated with a layer of silica to prevent interaction with hydrogen sulfide.

The FBR was installed in the trace-gas contaminant testing sample line as shown in Figure 16. The FBR is located downstream of the heated particulate filter and upstream of the tar condenser and trace-gas measurement sample point. The particulate filter and FBR were mounted in a heated enclosure. The enclosure temperature was set at a predetermined level appropriate for maximum sorbent reactivity.

\subsection{Tar analysis methodology}

The producer gas generated by various kinds of biomass gasification processes is inevitably contaminated with particulates and heavy hydrocarbons (tars), which, if not removed efficiently, would damage subsequent equipment and cause several operational problems. It has been a major reason which is responsible for the failure of large number of gasification projects 


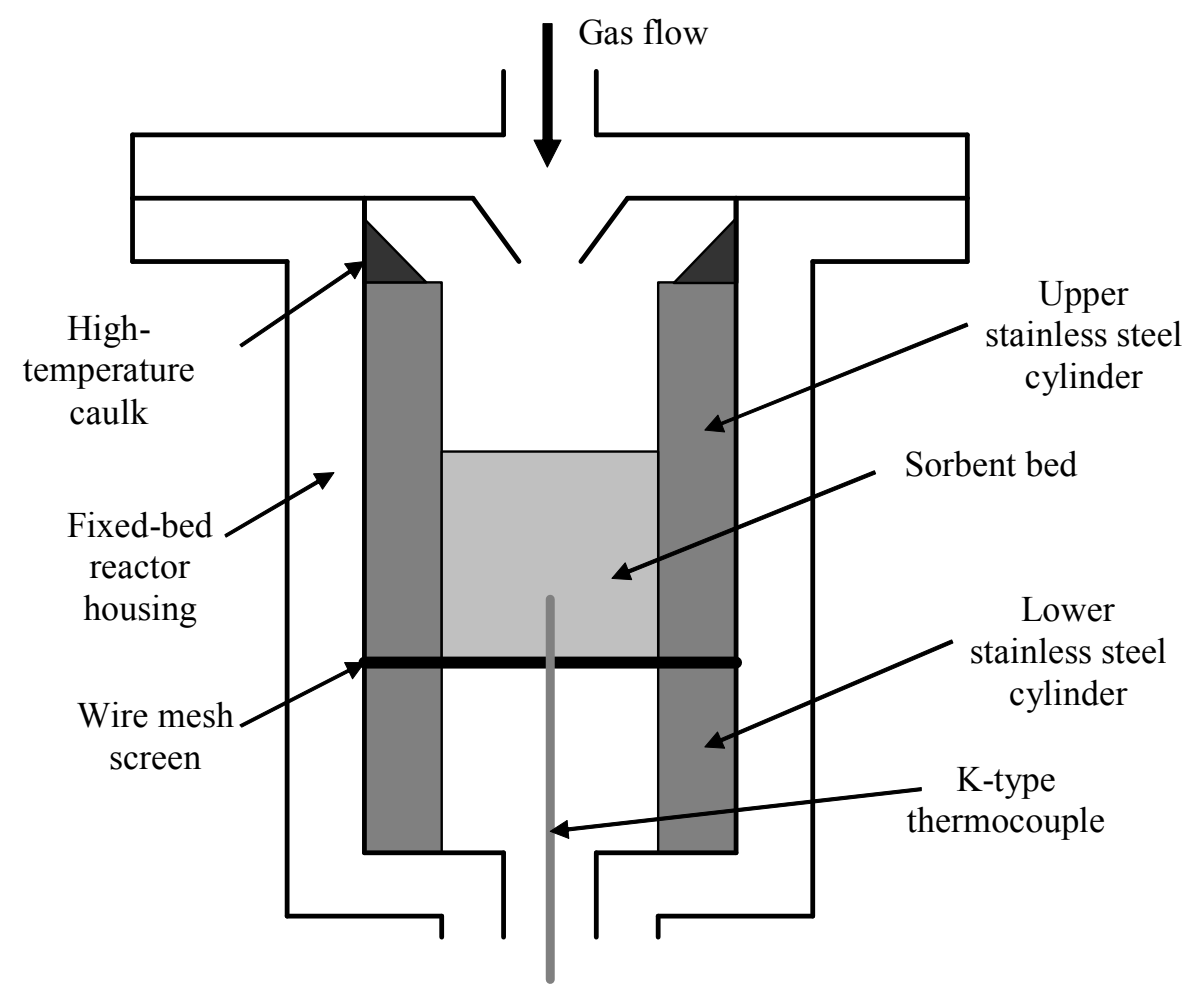

Figure 15 Schematic of sorbent reactor 


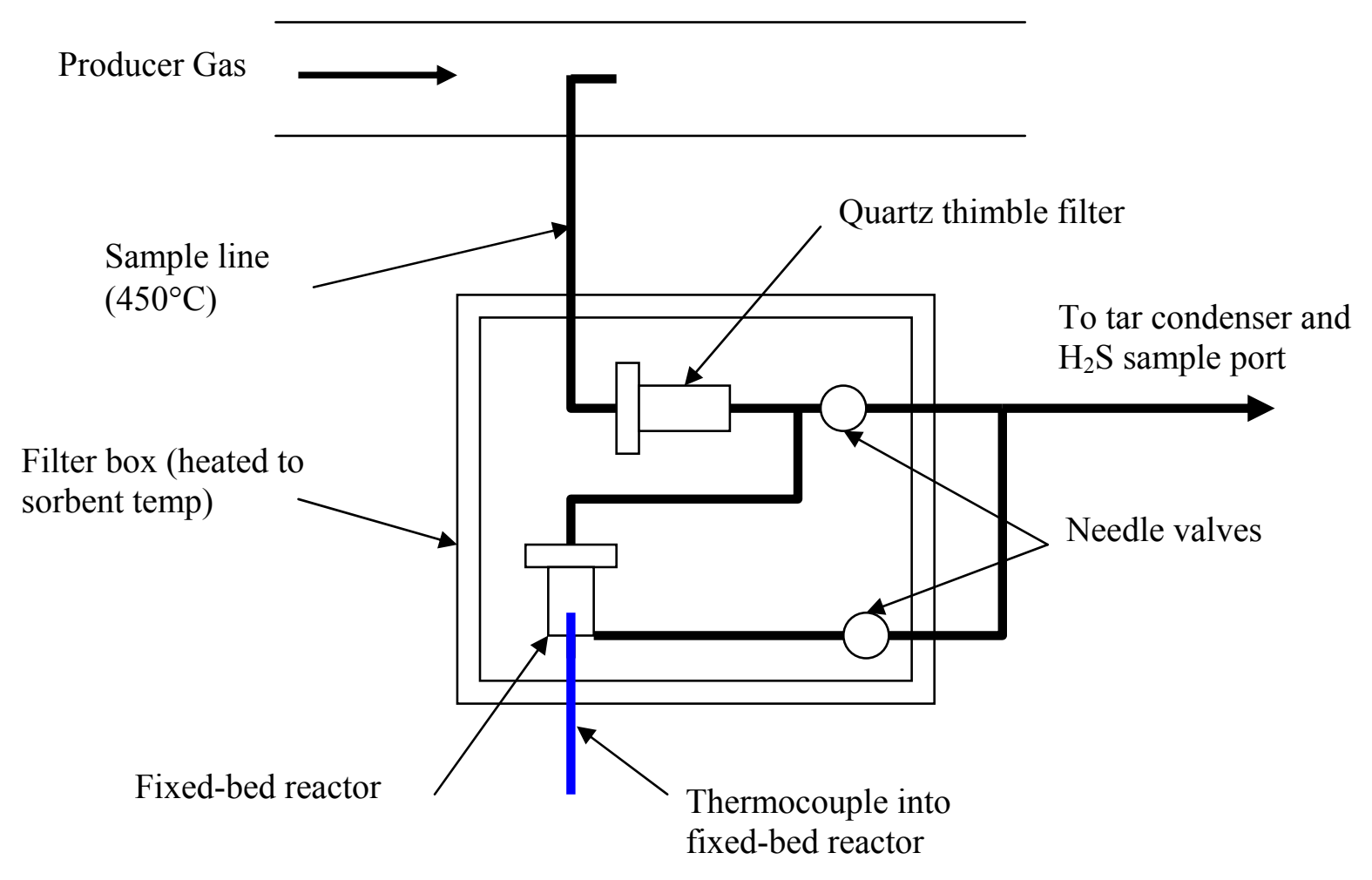

Figure 16 Schematic of fixed-bed reactor set-up 
throughout the world. Thus, intensive gas cleaning is required. The determination of the tar content in the gas is, therefore, of major importance with respect to gas quality and utilization.

In the field of biomass gasification, a large variety of sampling and analysis methods have been developed to determine the level of tar loading in the biomass-derived gasification producer gas, which makes comparison among different researchers and manufacturers very difficult. Traditionally, tar measurements are performed widely by methods based on cold trapping in impinger trains with a solvent and subsequent laboratory analysis.

In an effort to improve accuracy and repeatability of tar measurements, researchers within the Working Group of the Biomass Gasification Task of the IEA Bioenergy Agreement developed a protocol for sampling and analysis of tar and particulates from biomass gasifiers. ${ }^{17}$ This protocol, originally designed to use dichloromethane (DCM) as a tar solvent, has subsequently been modified to use less toxic solvents. However, the work reported here employs the original protocol because of it allows easy separation of water from the tars dissolved in the DCM and because of the skill developed at ISU in performing this protocol.

Regardless of solvent used, the IEA protocol has several prominent shortcomings. Handling of organic solvents requires special skills and extra attention by gasifier operators to avoid toxic exposure and environmental contamination. In the case of DCM, this involves wearing properly fitted organic vapor respirator and gloves and restricting exposure of unprotected personnel working near the areas of sampling and analysis. In addition, the IEA protocol requires considerable time for equipment set-up, sampling, analysis, and clean-up. It is difficult to obtain more than one data point every few hours and most operators would find it inconvenient to obtain more than one data point per day. Finally, the procedure requires extreme operator skill, which results in considerable variation in results among less skilled operators. Thus, an important goal of this work was to develop an alternative method for measuring tar in producer gas that would meet industry's need for a simple and accurate procedure for routine applications. The method developed employs a modified household pressure cooker operated to condense tars while maintaining water in the vapor state and combined with a simple gravimetric measurement. This "hot condenser" method simplifies the sampling and analysis process significantly and reduces the time needed for sampling and analysis. It may also have prospects for continuous tar measurement.

\section{$\underline{\text { IEA Protocol }}$}

This protocol was used to measure tar during the steam reforming experiments performed as part of this research project (results reported in Section 3.3). We only briefly outline this procedure since details can be found in the literature. ${ }^{17}$

The gas sampling system used for the IEA Protocol is illustrated schematically in Figure 17 (refer to the lower flow path). Gas drawn from the slipstream passed through a heated particulate filter followed by a series of six impinger bottles placed in cooling baths. The first four bottles were immersed in an ice bath while the last two bottles were immersed in an acetone/dry ice bath. The first and sixth bottles were filled with glass beads, while the second, third and fourth bottles were filled with dichloromethane. The fifth bottle was filled with both glass beads and dichloromethane. Gas leaving the impinger train passed through a vacuum 


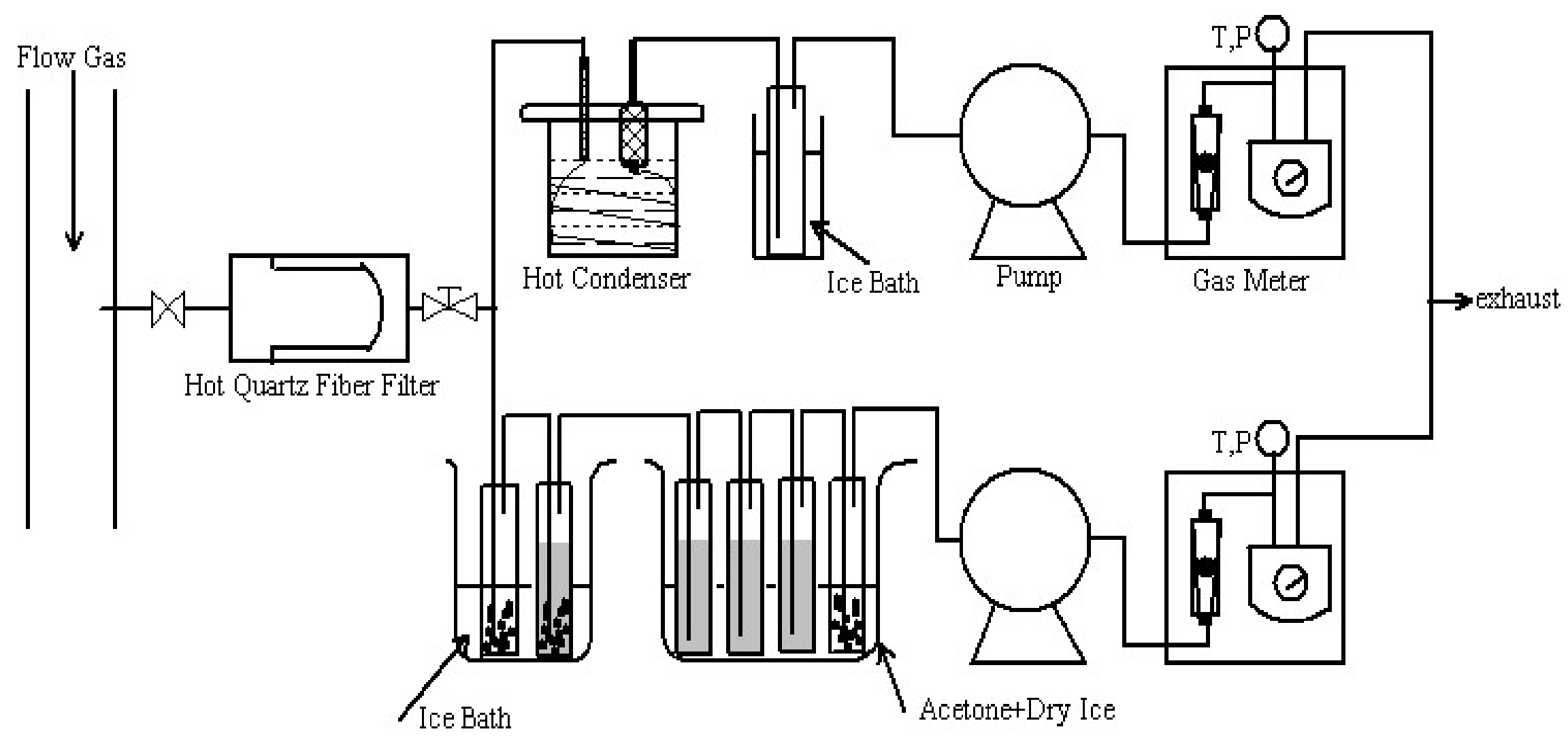

Figure 17 Schematic of the tar sampling and collection system (the upper flow path is the hot condenser system developed at ISU and the lower flow path is the impinger system using the IEA Tar Sampling Protocol) 
pump before exiting through a wet test meter to accurately determine the total (dry) gas volume sampled.

Gas samples were taken before the guard bed and after the catalytic reactor to provide information about overall system performance. Gas sampling was done every half-hour after steady operation of the gasifier and catalytic reactors were achieved. Gas samples were analyzed off-line by gas chromatography using a Varian Micro-GC CP-2003 Quad equipped with Molsieve 5A BF, Poroplot Q, and CP-Sils CB columns and a thermal conductivity detector with argon as carrier gas for the first column and helium as carrier gas for the second and third columns. The first column gave $\mathrm{H}_{2}, \mathrm{O}_{2}, \mathrm{~N}_{2}, \mathrm{CH}_{4}$, and $\mathrm{CO}$ concentrations; the second and third columns yielded $\mathrm{CO}_{2}, \mathrm{C}_{2} \mathrm{H}_{4}$ and some light hydrocarbons.

At the completion of a test, dichloromethane was rinsed through gas lines connected to the impingers to remove any tar condensed in them. This rinse liquid and impinger liquid were combined and refrigerated until tar analysis was performed.

Two types of analysis were performed on these tar samples: evaporation at $105^{\circ} \mathrm{C}$ and distillation at $75^{\circ} \mathrm{C}$. In either case, analysis began by filtering out solids from the sample mixture and decanting water from it. Evaporative analysis was the simpler of the two analyses performed and yielded tar values in good agreement with traditional methods of measuring "heavy $\operatorname{tar} " 18$. This analysis consists of pouring $50 \mathrm{ml}$ of DCM/tar mixture in a ceramic dish, letting it stand in a fume hood overnight, moving it to a heating chamber at $105^{\circ} \mathrm{C}$ for 1 hour, and recording the weight of the remaining residue. From knowledge of the total gas flow through the sampling system, tar concentration in the producer gas can be obtained, which we shall refer to as "heavy tar."

The second method of analysis is based on distilling $50 \mathrm{ml}$ of the DCM/tar mixture in a water bath maintained at $75^{\circ} \mathrm{C}$ for 30 minutes. This distillation produces two fractions of hydrocarbons: light hydrocarbons (still dissolved in the distilled dichloromethane) and the distillation residue. In addition, the decanted water contains a third fraction of dissolved hydrocarbons referred to as water-soluble hydrocarbons. These three fractions were sent out for total organic carbon (TOC) analysis, which is useful in estimating the amount of steam required to convert the hydrocarbons in tar to carbon monoxide and hydrogen.

\section{$\underline{\mathrm{DCM} / \text { Tar Storage Protocols }}$}

Although not specified in the IEA protocol, anecdotal evidence in our laboratory suggested that the age of a tar/DMC sample at the time of analysis affected the measured value of tar concentration. Since it is common practice to store tar/DCM samples obtained from a gasification trial for hours or even several days before distilling or evaporating it to determine tar content, we decided to test two different storage protocols for tar/DCM samples and determine whether the age of a sample affected the apparent tar concentration, as analyzed by the IEA protocol.

To perform this evaluation, switchgrass was gasified at several different temperatures and the IEA protocol, previously described in Section 2.8, was used to collect tar and water from the producer gas in dimethyl chloride. The test conditions are indicated in Table 6. 
Table 6 Gasifier and sampling conditions for obtaining tar samples for evaluating the effect of sample age on measurement accuracy

\begin{tabular}{cccc}
\hline $\begin{array}{c}\text { Gasification } \\
\text { Trial }\end{array}$ & $\begin{array}{c}\text { Gasification } \\
\text { Temperature } \\
\left({ }^{0} \mathrm{C}\right)\end{array}$ & $\begin{array}{c}\text { Gas Sampling } \\
\text { Temperature } \\
\left({ }^{0} \mathrm{C}\right)\end{array}$ & $\begin{array}{c}\text { Sample Storage } \\
\text { Protocol }\end{array}$ \\
\hline 1 & 736 & 23.0 & Single Flask \\
2 & 670 & 23.7 & Single Flask \\
3 & 650 & 32.5 & Multiple Flask \\
4 & 732 & 30.6 & Multiple Flask \\
5 & 788 & 27.2 & Multiple Flask
\end{tabular}

Two different storage protocols were evaluated: single flask storage for gasification trials 1 and 2 and multipe flask storage for gasification trials 3-5. The single flask storage protocol was as follows:

- The tar/water/DCM solution was filtered to remove fine particulate, allowed to stand long enough for the water, which is immiscible in DCM, to separate and then the water decanted. The resulting tar/DCM solution was poured in a single $1000 \mathrm{ml}$ flask, which was refrigerated at approximately $3{ }^{\circ} \mathrm{C}$ until analyzed, as specified by IEA protocol.

- Half an hour before analysis, the flask was removed from the refrigerator and allowed to come to room temperature (about $20^{\circ} \mathrm{C}$ ). From it as removed $100 \mathrm{ml}$ of tar/DCM sample, sufficient for four replications of tar analysis. The storage bottle was immediately resealed and returned to the refrigerator until the next time interval had passed and a sample was required.

The multiple flask storage protocol was as follows:

- The tar/water/DCM solution was filtered to remove fine particulate, allowed to stand long enough for the water, which is immiscible in DCM, to separate and then the water decanted. The resulting tar/DCM solution was subdivided and poured into a suitable number of $100 \mathrm{ml}$ flasks, one for each time interval to be evaluated, which were refrigerated at approximately $3^{\circ} \mathrm{C}$ until analyzed, as specified by IEA protocol.

- Half an hour before analysis, the flask containing the tar sample to be analyzed was removed from the refrigerator and allowed to come to room temperature (about $20^{\circ} \mathrm{C}$ ).

The reasons for these separate storage protocols are explained in the results section of this report. The analysis protocol, common to both storage protocols, employed the following procedures:

- Evaporating dishes were prepared by drying them in an oven at $105^{\circ} \mathrm{C}$ for half an hour, weighting them to obtain tare weights, and storing them in a desiccator at least two hours before use. The dishes were weighed on the electrical balance with the precision of $0.0001 \mathrm{~g}$. 
- $25 \mathrm{ml}$ tar/DCM solution was pipetted to an evaporating dish. Four parallel determinations were made for each sample for each time interval.

- The evaporating dishes containing the tar/DCM samples were moved to a fume hood maintained at room temperature for eight hours and then are moved to the fumed heating chamber at $105^{\circ} \mathrm{C}$ for one hour. The dishes were then removed and cooled to room temperature in a desiccator for two hours before being weighed.

- IEA protocol was used to convert these gravimetric measurements into tar concentrations $\left(\mathrm{g} / \mathrm{m}^{3}\right.$ at $0^{\circ} \mathrm{C}$ and $\left.1 \mathrm{~atm}\right)$ in the producer gas stream.

- Blank tests were carried out on pure dichloromethane for comparison to the tar/DCM samples.

\section{$\underline{\text { Hot Condenser Protocol }}$}

This protocol was developed after completion of steam reforming experiments and to date has only been used to validate the method and compare it against the IEA Protocol. The gas sampling system used for the hot condenser protocol is illustrated schematically in Figure 17 (refer to the upper flow path). Particulate content of less than $50 \mathrm{~g} / \mathrm{Nm}^{3}$ and tar content of less than $50 \mathrm{~g} / \mathrm{Nm}^{3}$ in producer gas were assumed in designing the tar condenser system although the method is probably suitable for particulate and tar concentrations twice these levels if shorter sampling intervals are employed. The method collects organic compounds with boiling points higher than about $100^{\circ} \mathrm{C}$. Thus, the method would collect toluene (boiling point of $110^{\circ} \mathrm{C}$ ) but not benzene (boiling point of $80^{\circ} \mathrm{C}$ ). These "heavy tars" should correspond closely to the "heavy tars" collected by the evaporative method of the IEA protocol (which is performed at $105^{\circ} \mathrm{C}$ ). Thus, subsequent comparisons between the two protocols employ the evaporative rather than the distillation version of the IEA protocol.

The sampling system for the hot condenser protocol consists of:

- a hot quartz fiber filter for particulate removal (shared with the IEA protocol system)

- a household pressure cooker with Swagelok fittings added to the lid to allow the gas sample to flow through it during a test

- a electric hot plate to heat the pressure cooker at 101 to $105^{\circ} \mathrm{C}$ during a test

- twenty-foot of Santoprene tubing installed in the pressure cooker and connected to the inlet fitting (selected for its excellent chemical and high-temperature resistance)

- a stainless steel canister containing glass wool installed in the pressure cooker and connected to the Santoprene tubing and the outlet fitting

- a chilled bottle for condensing water (and a small amount of low molecular weight organic compounds)

- a vacuum pump to draw producer gas through the hot condenser

- a rotameter, dry gas test meter, pressure gage, and thermocouple for measuring the total volume of gas (standard conditions) through the hot condenser during the sampling interval

The pressure cooker, properly operated, can be maintained at just above $100^{\circ} \mathrm{C}$, which keeps most of the water vapor from condensing but allows most of the heavy tars to condense onto the inner surface of the Santoprene tubing. The canister of glass wool attached downstream of the Santoprene tubing is designed to collect tarry aerosols that exist in the producer gas. These can constitute a significant fraction of the total heavy tars collected. At the end of a test, both the 
Santoprene tube and on the canister of glass wool are removed and weighed to determine weight gain, which is assumed to be from heavy tar. This method does not collect light tars such as benzene and toluene.

The following procedures are used to prepare the hot condenser system and perform tar measurements:

- Isokinetic sampling in the gasifier duct is only required if measurement of particulate concentrations is desired. In this case, ISO 9096 methods for sampling flue gas should be followed.

- A $20 \mathrm{ft}$-length of Santoprene tubing is prepared by attaching 3/8" compression fittings to each end of the tubing. The tube is coiled in a $2000 \mathrm{ml}$ beaker which is placed in a furnace maintained at $110^{\circ} \mathrm{C}$ for at least 12 hours or until weight loss due to devolatilization of plasticizers in the tube is complete. Then devolatilized tube is allowed to acclimatize in a desiccator at room temperature for one or two hours.

- One hour before a test, the tube is removed from the desiccator and the ends connected via a $3 / 8$ " union fitting. The tubing and fittings are weighted with an analytical balance having an accuracy of $\pm 0.001 \mathrm{mg}$. This is the "tare" weight for the tubing and fittings.

- The aerosol capture canister is loaded with quartz or glass wool, and its tare weight determined. The wool should be dried in desiccator first and be packed loosely so that there is no more than 2" Hg vacuum when air is drawn through the packed canister with a vacuum pump.

- The tubing is shaped into a coil and installed in the pressure cooker. The coil is loosely secured with cable ties, but not so tightly as to kink or block the tubes. Care should be taken such that, once water is added to the pressure cooker, the tubing is submerged, which increases heat transfer and ensures tar condensation.

- The union fitting is removed from the tubing and one end connected to the inlet fitting of the pressure cooker and the other end connected to the inlet fitting of the aerosol collection canister. The other end of the canister is attached to the outlet fitting of the pressure cooker Anti-seize compound is applied to all fittings before making these connections. The pressure cooker is filled to about $75 \%$ capacity with distilled or deionized water and then the lid is sealed to the body of the pressure cooker. The steam escape value on the pressure cooker is adjusted to about $105^{\circ} \mathrm{C}$ and the cooker is placed on the electrical hot plate.

- Once the pressure cooker is up to operating temperature, sampling is initiated by starting the vacuum pump. The volumetric sampling flow rate is adjusted to that required for isokinetic sampling if particulate measurements. Otherwise, the flow rate should be set to about $2.5 \mathrm{~L} / \mathrm{min}$ (at STP) but not exceeding $5 \mathrm{~L} / \mathrm{min}$ (at STP). Sampling should be terminated if the vacuum pressure between the condenser and the pump significantly exceeds $5 " \mathrm{Hg}$. The pressure and temperature ahead of the gas meter is periodically recorded.

- When sampling is complete, the pressure cooker is removed from the hot plate, pressure allowed to relieve from the cooker, and the tubing removed, the ends secured together by the original union fitting used for the tare measurement to prevent weight loss from volatilization of the still hot tar.

- Once the tubing has cooled, the outside of the tubing is dried with a towel and further dried by placing the sealed tube in the desiccator for half an hour. The tubing and fittings 
(including the union fitting) are weighed. The difference between this measurement and the tare measurement is the mass of condensed tar in the tubing.

- In a similar manner the aerosol capture canister is dried and weighted. The difference between this measurement and the tare measurement is the mass of aerosol tar captured in the glass wool.

- The total tar is the sum of the masses obtained from the previous two steps. The tar concentration is calculated by dividing the mass of tar by the total gas volume passing through the hot condenser during sampling, as measured by the dry test meter.

\subsection{Multi-mode fluidized reactor}

As part of the project, a new thermochemical reactor was designed and constructed that can function as either a combustor or gasifier. This fluidized bed reactor has several modes of operation: bubbling fluid bed combustor, bubbling fluid bed gasifier, circulating fluid bed combustor, or circulating fluid bed gasifier.

The fluid bed reactor and associated feed vessels were built as ASME pressure vessels, enabling bubbling fluid bed operation to $75 \mathrm{psig}$. The modest pressure rating offers several benefits. Reactor throughput increases linearly with the ratio of absolute operating pressure to atmospheric pressure. Therefore operation at $65 \mathrm{psig}$ (a value slightly lower than the pressure vessel rating) increases capacity approximately fivefold ( $80 \mathrm{psia} / 16 \mathrm{psia})$. The nominal feed rates are shown in Table 7. Gasification at moderate pressures will alleviate one or more stages of syngas compression for subsequent catalytic synthesis reactions. Vessel construction using standard 150\# fittings is not significantly different than construction of an 'atmospheric' rated vessel. Biomass feed at moderate pressures much simpler than for biomass fed at high pressures $(>10$ atmospheres).

Table 7 Nominal fuel feed rates for various operating modes and operating pressures

\begin{tabular}{|l|c|c|}
\hline Operation Mode & $\begin{array}{l}\text { Nominal biomass feed rate } \\
\text { at atmospheric pressure }\end{array}$ & $\begin{array}{l}\text { Nominal biomass feed rate } \\
\text { at elevated pressure* }\end{array}$ \\
\hline Bubbling fluid bed combustion & $10-30 \mathrm{lb} / \mathrm{hr}$ & $35-125 \mathrm{lb} / \mathrm{hr}$ \\
\hline Bubbling fluid bed gasification & $40-130 \mathrm{lb} / \mathrm{hr}$ & $200-650 \mathrm{lb} / \mathrm{hr}$ \\
\hline Circulating fluid bed combustion & $70-175 \mathrm{lb} / \mathrm{hr}$ & $\mathrm{NA}^{* * * *}$ \\
\hline Circulating fluid bed gasification & $350-900 \mathrm{lb} / \mathrm{hr}$ & $\mathrm{NA}^{* * *}$ \\
\hline
\end{tabular}

Atmospheric pressure is nominally 2 psig

${ }^{* *}$ Atmospheric pressure is nominally 65 psig

${ }^{* * *}$ Circulating mode only possible at atmospheric pressure

The multi-mode fluidized reactor is illustrated in Figure 18. The inner diameter of the vessel is 12-in from top-to-bottom. Three 6-ft long sections make up the body of the vessel for an overall fluid bed height of 18 -ft. Two feed ports accommodate two biomass feed injection elevations. The lower feed elevation will typically be used for bubbling fluid bed operation but can also be used for circulating operation. The upper feed elevation is intended for use during circulating operation. Carbon conversion typically increases during circulating gasification by creating an oxidation zone between the fluidization gas nozzles and the biomass 


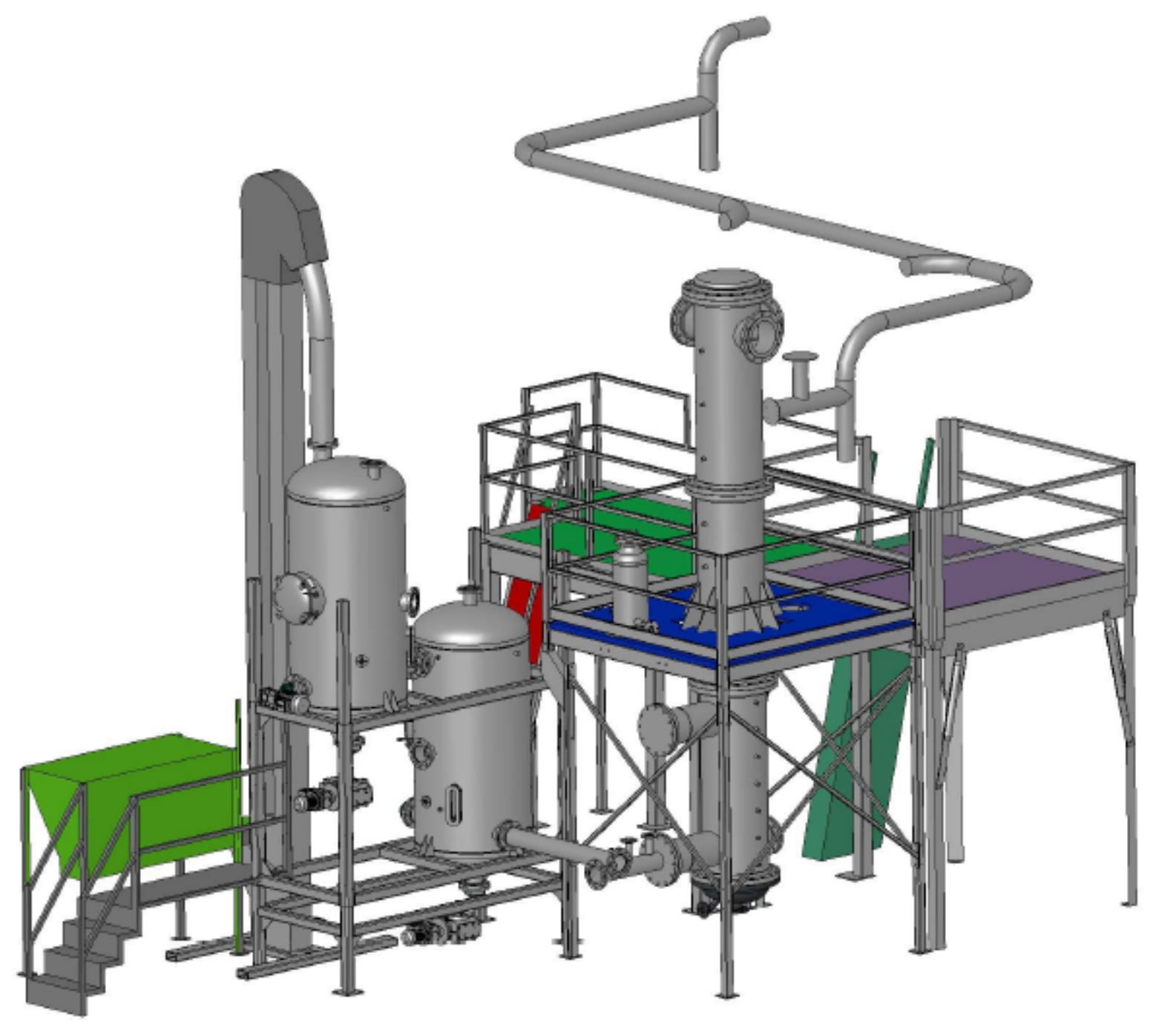

Figure 18 Multi-mode fluidized bed reactor system 
injection nozzle. The recirculation of bed media and biomass char near the bottom of this zone results in a one-second residence time for char/oxygen contact.

Pressurized bubbling bed operation is accomplished using a pressure modulation valve that restricts gas flow exiting the reactor. For circulating bed operation, this modulation valve is replaced with a cyclone separator, a standpipe, and L-valve to separate elutriated bed media, which is reinjected into the bottom of the fluid bed just above the distributor and below the biomass feed nozzle.

The biomass feed system consists of two pressure vessels in series. The 'metering bin' operates at process pressure at all times and has two different sized, speed controlled screw augers that meter biomass into a high speed injection auger. The 'cycling bin' periodically operates between atmospheric pressure and process pressure. At atmospheric pressure, the discharge of the cycling bin is isolated from the metering bin by a valve. Another valve at the inlet of the cycling bin opens and a fuel receiving hopper and bucket elevator deliver biomass to fill the cycling bin. When the cycling bin is full the inlet valve closes, the bin is brought to process pressure, the valve isolating the cycling bin from the metering bin opens, and biomass is transferred, filling the metering bin.

\section{Experimental Results}

\subsection{Ballasted gasifier trials}

Numerous experiments were conducted in order to characterize the operation of the ballasted gasification cycle in a fluidized bed. One set of experiments consisted of steam cooling tests, in which no biomass was fed to the reactor, while other experiments consisted of ballasted biomass gasification tests. The purpose of the steam cooling testing was to obtain bed temperature profiles during the cooling period to compare to a model of the ballasted gasifier, which is described in Chapter 4. All experiments were executed with ballast tubes present in the reactor. Some of these results have been reported in Smeenk et al. ${ }^{19}$ and Brown et al. ${ }^{20}$

\section{$\underline{\text { Reactor cooling time and cooling temperature profiles }}$}

The purpose of the latent-heat ballast is to increase the energy stored within the bed and allow a greater quantity of biomass to be processed during the pyrolysis phase of ballasted gasification. The additional advantage of the latent-heat ballast is to increase the average bed temperature during the pyrolysis period. The extent to which this temperature is increased can be determined by examining the temperature profiles during the phase. Reactor cooling time and temperature profiles were obtained for both steam cooling tests, in which no biomass was fed to the reactor, and the pyrolysis phase of ballasted gasification.

\section{$\underline{\text { Steam cooling tests }}$}

The purpose of the steam cooling tests is to provide experimental data to verify the bed temperature profiles predicted by ballasted gasifier model described in Chapter 4 . The heat 
transfer processes that occur within the reactor during pyrolysis are greatly complicated by the chemical energy absorbed and released as the multiple chemical reactions associated with pyrolysis proceed. This complex chemistry is not well understood, and to better understand the heat transfer processes not associated with pyrolysis chemistry, cooling tests were performed without feeding biomass into the reactor. With the chemical processes removed, all heat loss was attributed to one of three sinks: heat loss to fluidizing steam, heat loss to purge gas and heat loss through the composite wall.

Five steam cooling tests were performed. The steam flow rate was held constant at $90 \mathrm{~kg} / \mathrm{hr}$ for the first three of these tests, while the steam flow rate was adjusted to $100 \mathrm{~kg} / \mathrm{hr}$ and $82 \mathrm{~kg} / \mathrm{hr}$ for the fourth and fifth tests, respectively. The purge gas flow rate was set to $30.6 \mathrm{Nm}^{3} / \mathrm{hr}$. The resulting bed temperature profiles during the steam cooling tests are shown in Figure 19. The bed temperature profile was determined by averaging the measurements of the ten thermocouples placed within the fluidized bed.

The results fall in the order that one would expect, with cooling times decreasing as steam flow rate is increased, with the exception of the first test performed at a steam flow rate of $90 \mathrm{~kg} / \mathrm{hr}$. This test was the first test performed upon the completion of the start up process, and the reactor may not have yet reached quasi-steady state operation. The temperature profiles of the remaining runs completed at a steam flow rate of $90 \mathrm{~kg} / \mathrm{hr}$ lie virtually on top of one another, so disregarding the first run during analysis is justified. Surprisingly little variation exists as the steam flow rate is adjusted. The time required for the average bed temperature to fall from 1172 $\mathrm{K}$ to $922 \mathrm{~K}$ is 31.8 minutes when the steam flow rate was $90 \mathrm{~kg} / \mathrm{hr}$. This time increased only 1.5 minutes to 33.3 minutes when the steam flow rate was reduced to $82 \mathrm{~kg} / \mathrm{hr}$, while it decreased just 0.8 minutes to 31.0 minutes when the steam flow rate was increased to $100 \mathrm{~kg} / \mathrm{hr}$. This lack of variation suggests that losses through the wall and losses due to the heating of cold purge gas are on the same order of magnitude as the losses due to the heating of the steam during the cooling period.

The ballasted gasifier model predictions of the bed temperature profile were compared to steam cooling test data for all three steam flow rates. The results of the modeling of the steam cooling test with a flow rate of $82 \mathrm{~kg} / \mathrm{hr}$ are shown in Figure 20, while results from steam cooling tests with steam flow rates of $90 \mathrm{~kg} / \mathrm{hr}$ and $100 \mathrm{~kg} / \mathrm{hr}$ are illustrated in Figure 21 and Figure 22, respectively.

From the graphs, the model follows the behavior of the fluidized bed fairly well during the steam cooling periods. Further, the model shows the same slight variation in cooling time that was seen in experimental data. The model showed the ability to predict bed temperatures within 30 $\mathrm{K}$, when bed temperatures were on the order of $1000 \mathrm{~K}$, an error of less than $3 \%$ of the absolute temperature.

\section{$\underline{\text { Pyrolysis tests }}$}

In order to determine the bed temperature profiles during the pyrolysis of biomass, temperature data was recorded during the pyrolysis phase of the ballasted gasification cycle. The fluidized bed reactor was operated for five complete cycles. The feedstock used for these tests was corn. The biomass feed rate and steam flow rate were set to $180 \mathrm{~kg} / \mathrm{hr}$ and $90 \mathrm{~kg} / \mathrm{hr}$, respectively. The 
purge gas flow rate was set to $24 \mathrm{Nm}^{3} / \mathrm{hr}$. The resulting bed temperature profiles during the steam cooling tests are shown in Figure 23. Again, the bed temperature profile was determined by averaging the measurements of the ten thermocouples placed within the fluidized bed.

As the operating parameters were not altered during the experiment, little deviation is expected in the bed temperature profiles, and this is indeed the behavior seen in the experimental data. At any given time during the pyrolysis phase, the profiles of the five cycles varied less than $10 \mathrm{~K}$ 


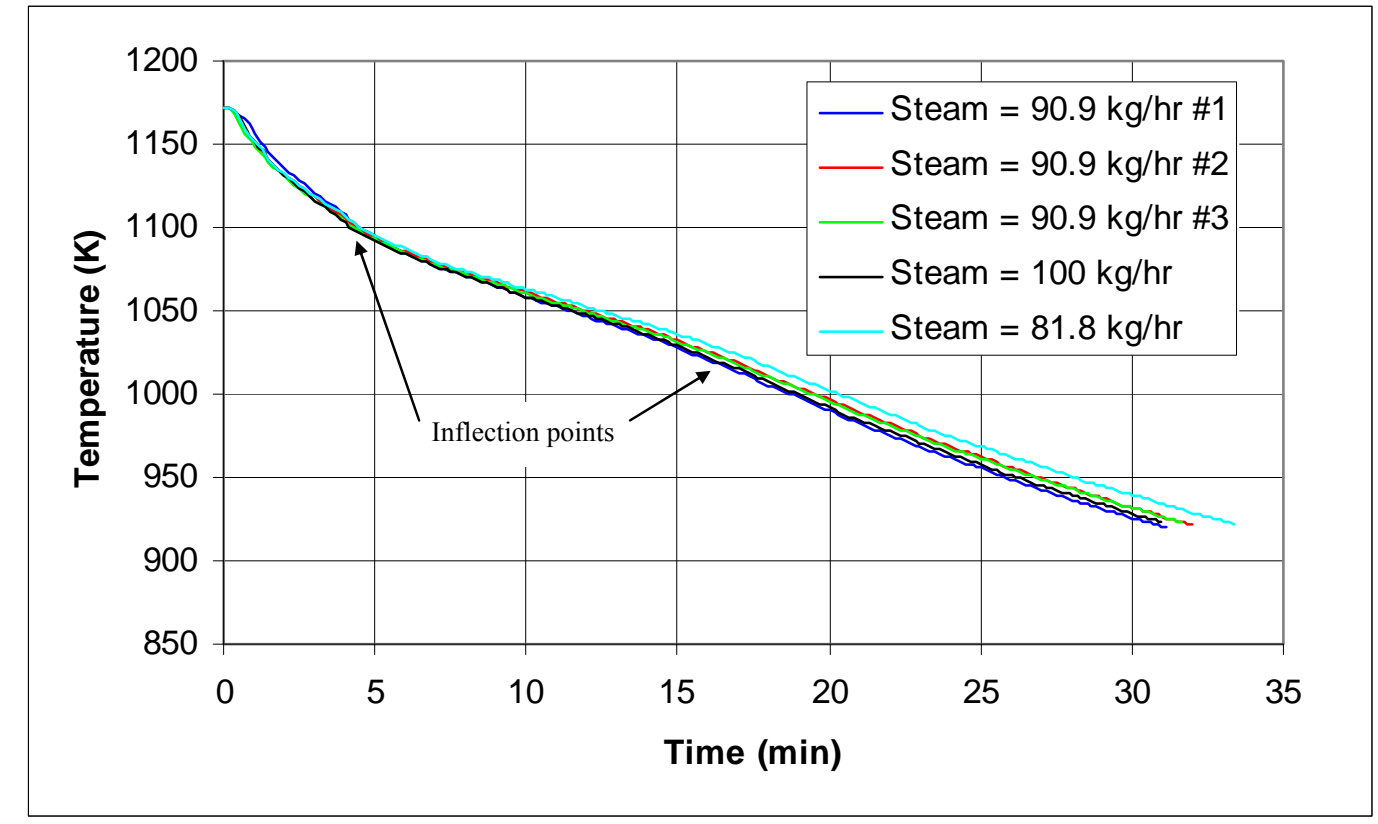

Figure 19 Bed temperature profiles during steam cooling for various steam flowrates 


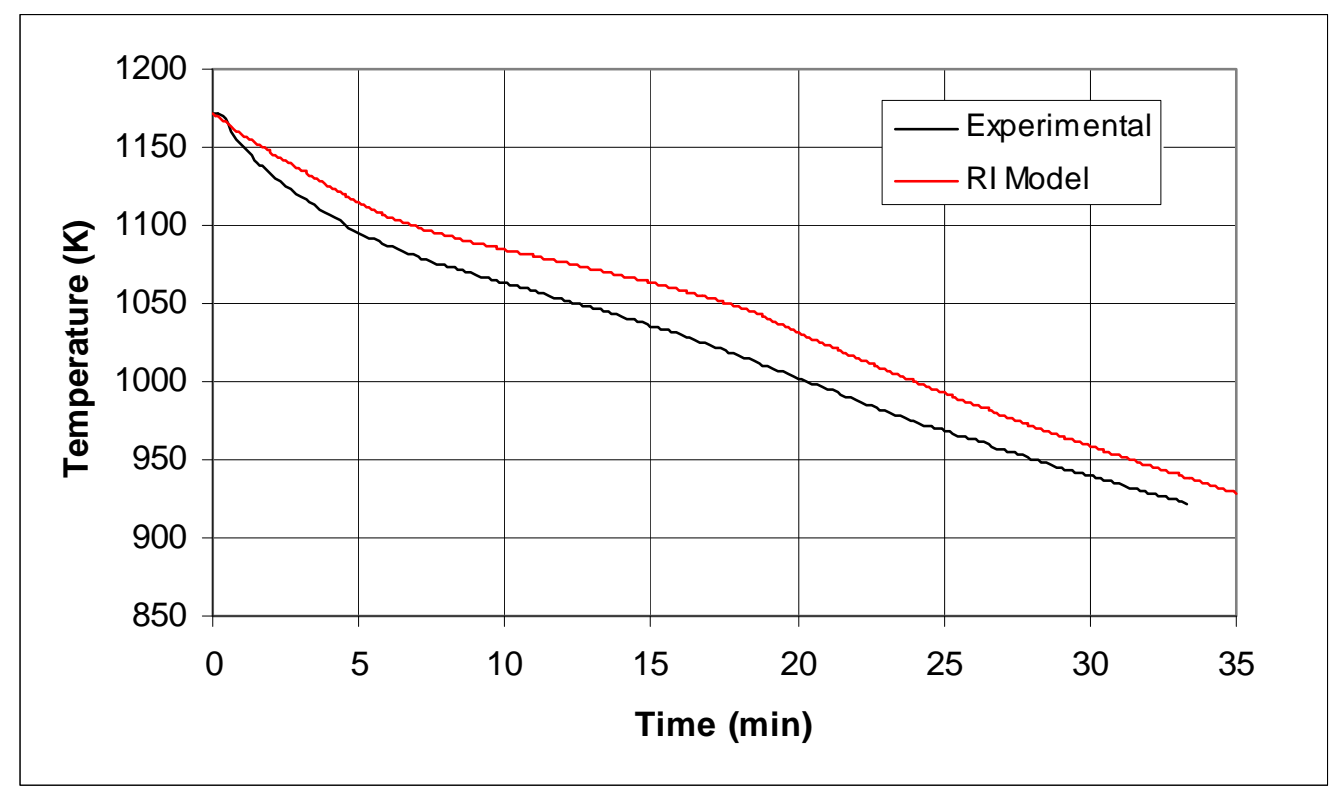

Figure 20 Bed temperature profiles during steam cooling tests (flowrate $=81.8 \mathrm{~kg} / \mathrm{hr}$ )

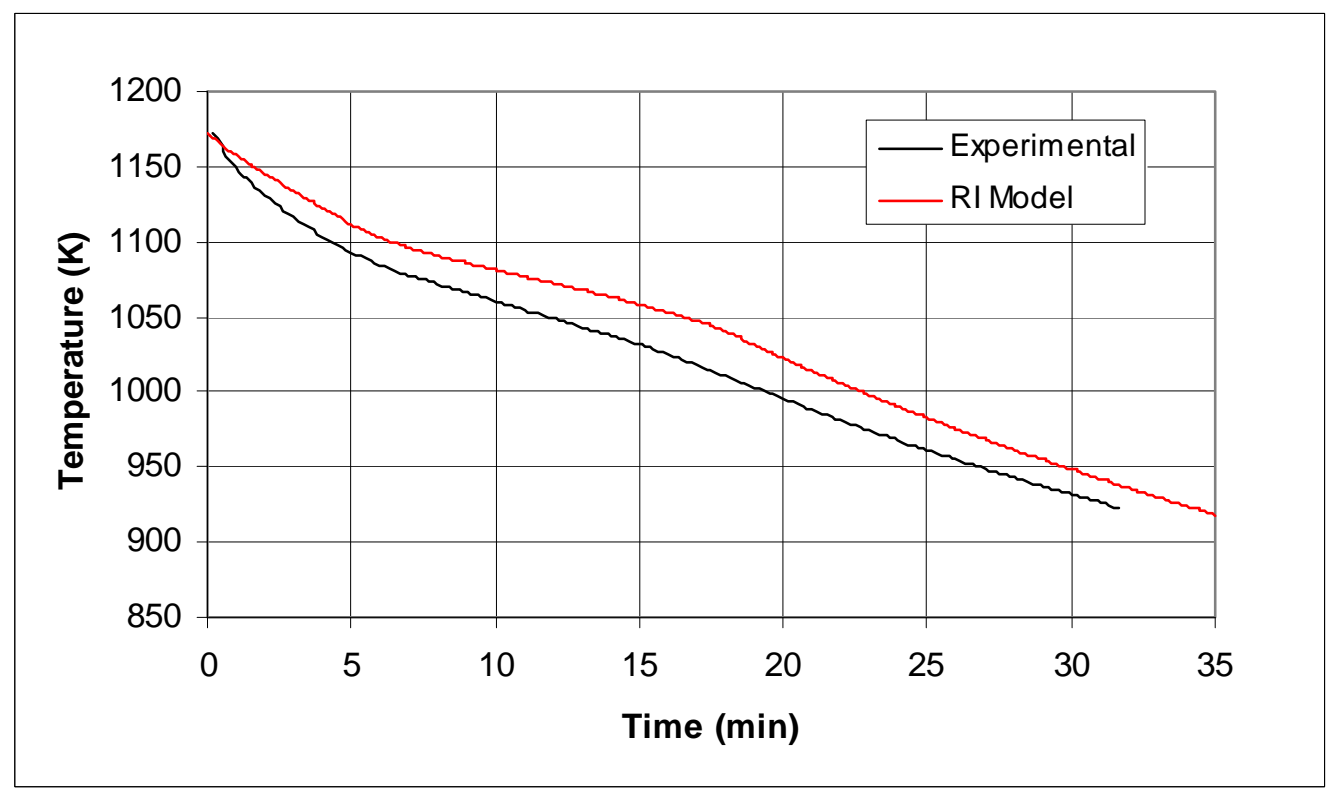

Figure 21 Bed temperature profiles during steam cooling tests (flowrate $=90.9 \mathrm{~kg} / \mathrm{hr}$ ) 


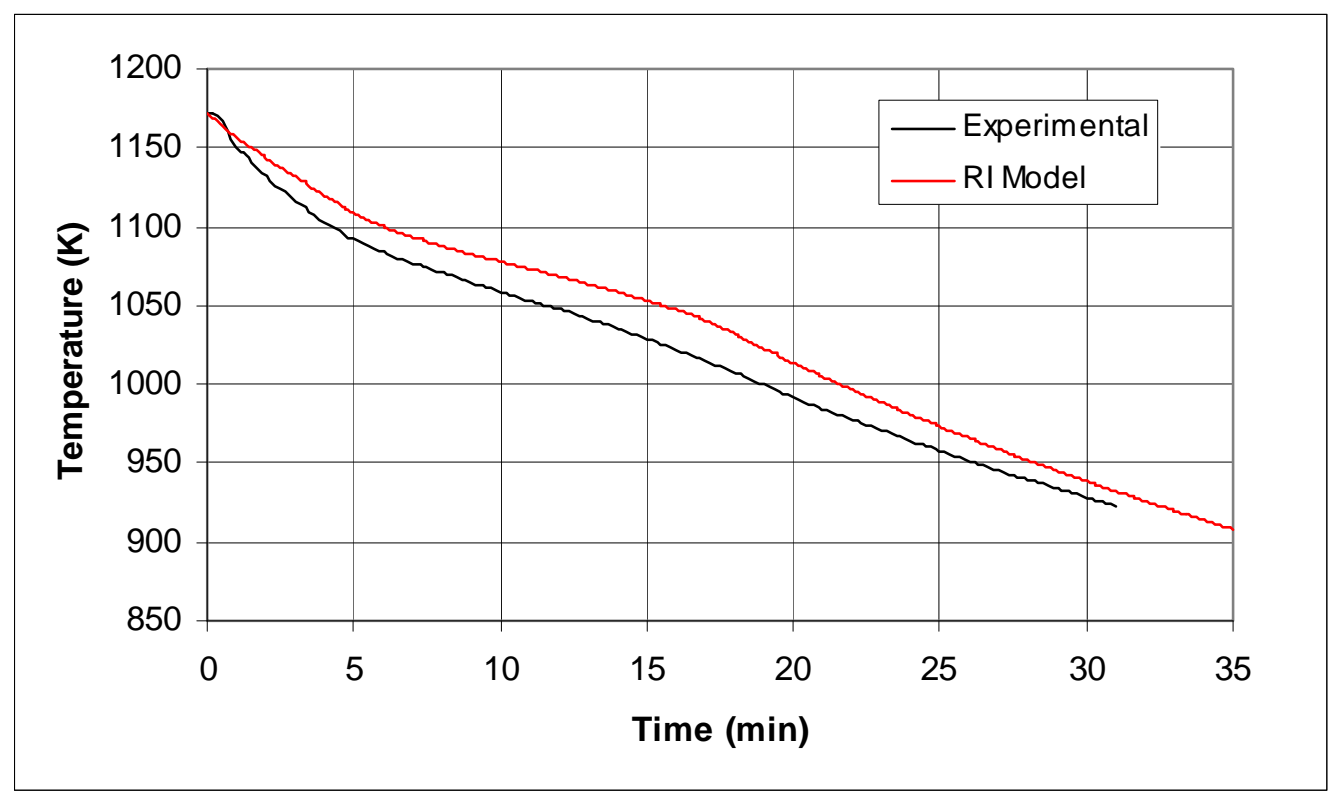

Figure 22 Bed temperature profiles during steam cooling tests (flowrate $=100.0 \mathrm{~kg} / \mathrm{hr}$ )

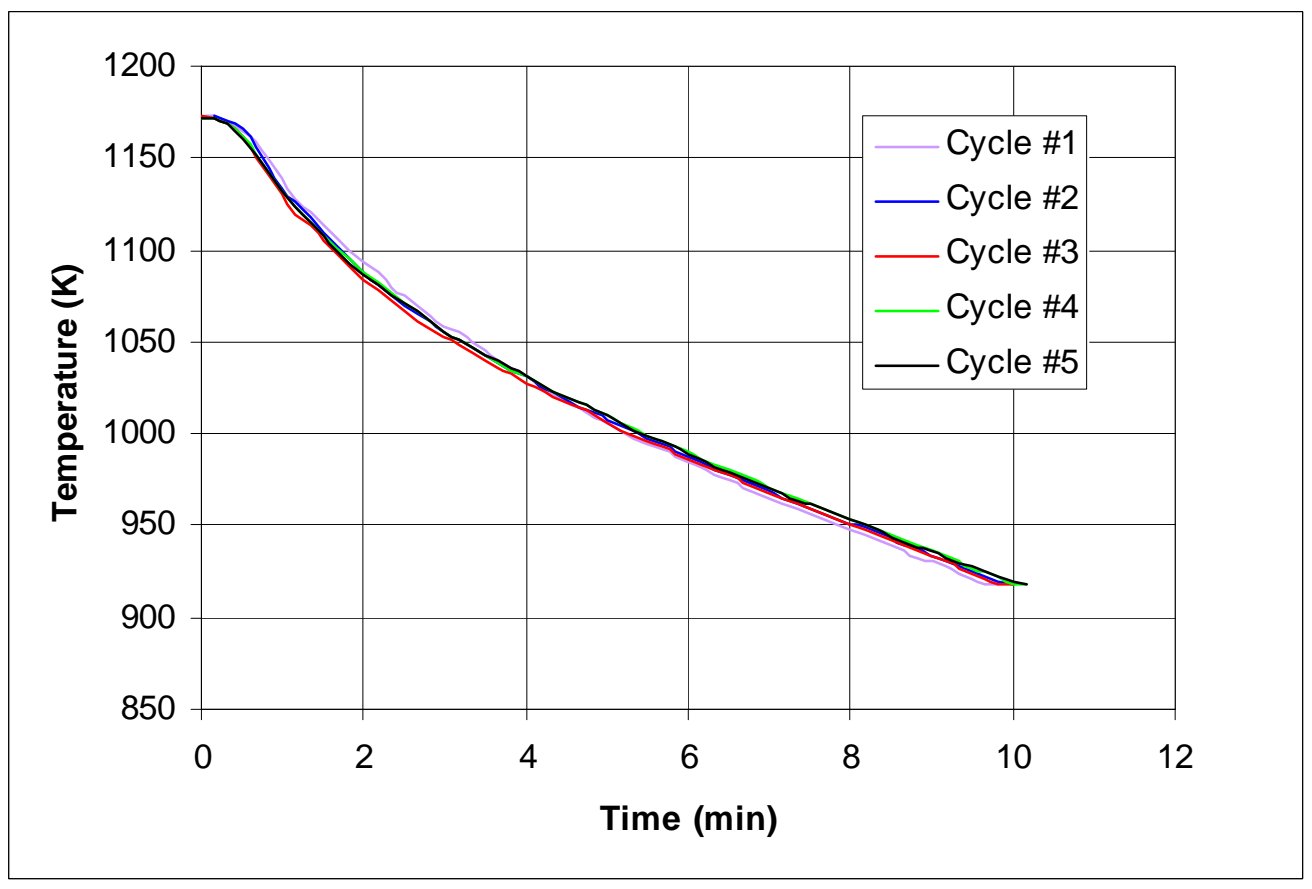

Figure 23 Bed temperature profiles during pyrolysis phase of ballasted gasification 
(while actual temperatures were on the order of $1000 \mathrm{~K}$ ). The time required for the bed to cool from $1172 \mathrm{~K}$ to $922 \mathrm{~K}$ ranged from 9.5 minutes to 9.8 minutes, a difference of about 20 seconds. This lack of variation indicates a consistent behavior of the entire system from cycle to cycle.

While the bed temperature profiles obtained during steam cooling tests contain two relatively clear inflection points indicating the onset and conclusion of the phase-change period, the profiles obtained during pyrolysis testing contains no clear inflection points corresponding to the onset and completion of the phase-change period. As discussed previously, ideal behavior is seen only when the thermal energy losses from the fluidized bed are balanced by the transfer of thermal energy from the ballast tubes. Compared to the rate of energy loss during steam cooling, the endothermic chemical processes of pyrolysis greatly increase the rate of energy loss from the bed, while the heat transfer process from the tubes to the bed remains relatively unchanged. Thus, the bed behaves much less ideally during this period, and these non-ideal processes "hide" the inflection points that signal the beginning and end of the phase-change process.

Attempts to predict the bed temperature profiles through the pyrolysis phase of the ballasted gasification cycle cannot be done considering the physical heat transfer processes alone. The energy required by the chemical reactions that occur during the pyrolysis process is substantial. Previous research found that thermodynamic predictions of the producer gas composition are inaccurate as thermodynamic equilibrium is not achieved within the reactor. Thus, modeling of the bed temperature profiles cannot be accomplished until this chemical process has been characterized. This characterization must include a description of the gas composition and a quantification of the amount of gas produced.

\section{Combustion temperature profiles}

While the products of the pyrolysis phase contain the desired fuel gases, consideration of the behavior of the fluidized bed during the combustion process is crucial when determining the ultimate efficiency of the system. Thus, data was also recorded during combustion phases of steam cooling tests and pyrolysis tests. The biomass feed rate during the combustion phase of both sets of tests was $68 \mathrm{~kg} / \mathrm{hr}$. During steam cooling testing, the air flow rate was $144.4 \mathrm{Nm}^{3} / \mathrm{hr}$, and the purge gas flow rate was $30.6 \mathrm{Nm}^{3} / \mathrm{hr}$. The resulting bed temperature profiles are illustrated in Figure 24. During pyrolysis testing, the air flow rate was $161.4 \mathrm{Nm}^{3} / \mathrm{hr}$, and the purge gas flow rate was $23.8 \mathrm{Nm}^{3} / \mathrm{hr}$. The bed temperature profiles from pyrolysis testing are shown in Figure 25. It is important to note that the air used to fluidize the bed during the combustion phase of both sets of tests was not pre-heated. Like the pyrolysis phase, the time required to complete the combustion period is consistent, with combustion times of approximately 29 minutes during steam cooling and approximately 27 minutes during pyrolysis tests.

The most prominent feature of the bed temperature profiles is the brief temperature plateau as the bed reached an average temperature near $1060 \mathrm{~K}\left(1450^{\circ} \mathrm{F}\right)$. This temperature is too low to cause the melting of lithium fluoride, and this effect is not attributable to char burnout as it is present during steam cooling tests, in which no char is produced. The calcination of limestone does occur at this temperature, but the repetition of this effect would indicate that limestone undergoes the reverse process during pyrolysis. Further investigation is required to determine the source of this phenomenon. 


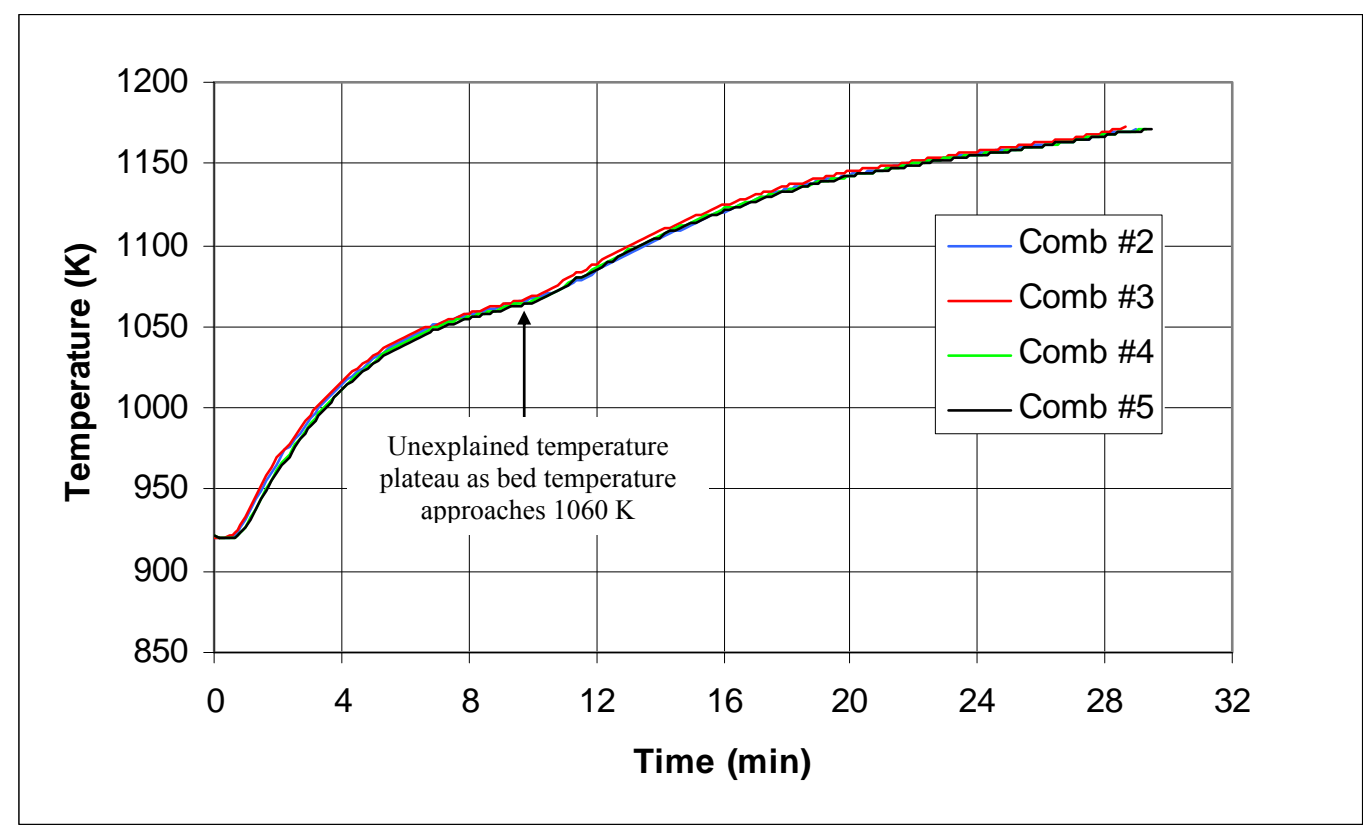

Figure 24 Bed temperature profiles during combustion phase of steam cooling tests

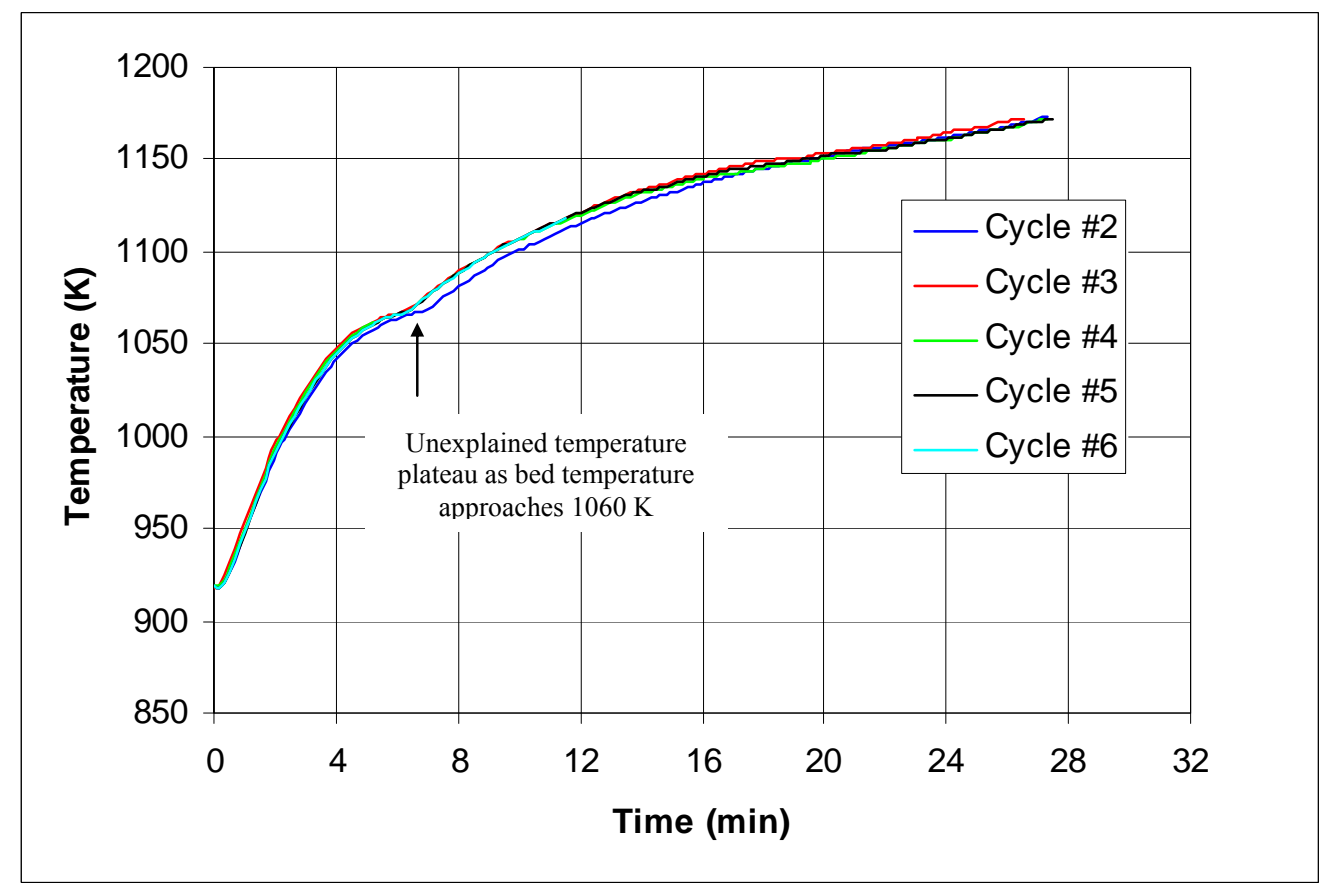

Figure 25 Bed temperature profiles during combustion phase of ballasted gasification 
At first glance, it is difficult to identify the phase-change period during the combustion phase. If the temperature profiles are re-examined, focusing on the end of the combustion period, the phase-change period becomes more evident. With the time scale changed to a range of 12-28 minutes and the temperature scale set to $1125-1175 \mathrm{~K}$, inflection points become apparent at the approximate times of 18 minutes and 23 minutes, as depicted in Figure 26. As the phase-change temperature of lithium fluoride is $1121 \mathrm{~K}$, this is the portion of the combustion phase that one would expect the phase-change process to occur. The time required for the melting process is approximately 7 minutes, shorter than the solidification process during steam cooling tests. This shorter phase-change time is due to the large quantities of thermal energy provided by the combustion process.

\section{Gas composition}

As mentioned previously, characterization of the producer gas stream is essential to predicting the heat transfer processes within the reactor during pyrolysis. Thus, the primary goal of the ballasted gasification testing was the acquisition of gas composition profiles throughout the pyrolysis phase. One of the principal limitations of the previous research was the inability of gas analysis systems to continuously monitor the changes in the gas composition as the pyrolysis phase proceeds. Only time-averaged compositions could be determined, which did not accurately characterize the dynamic chemical processes that occur during the pyrolysis phase of ballasted gasification. This limitation was addressed, and continuous emission monitors were available during the present study to continuously quantify the species of most interest: hydrogen and carbon monoxide. These continuous profiles were supplemented by intermittent determinations of other species within the gas matrix by a micro-GC. Continuous emission monitors (CEM) quantified the content of the following gases: hydrogen $\left(\mathrm{H}_{2}\right)$, carbon monoxide (CO), carbon dioxide $\left(\mathrm{CO}_{2}\right)$ and oxygen $\left(\mathrm{O}_{2}\right)$. A Varian micro-GC also determined the presence of these gases, as well as methane $\left(\mathrm{CH}_{4}\right)$, acetylene $\left(\mathrm{C}_{2} \mathrm{H}_{2}\right)$ and ethylene $\left(\mathrm{C}_{2} \mathrm{H}_{4}\right)$, ethane $\left(\mathrm{C}_{2} \mathrm{H}_{6}\right)$, propane $\left(\mathrm{C}_{3} \mathrm{H}_{8}\right)$ and nitrogen $\left(\mathrm{N}_{2}\right)$. All gas compositions were determined on a dry, tar-free basis. The CEM data throughout the testing period is illustrated in Figure 27.

One of the most striking features of Figure 27 is the repeatability of the gas composition through each phase of ballasted gasification, particularly for the final four cycles. During the first cycle, the $\mathrm{H}_{2}$ content of the gas is about 2 vol-\% lower during the initial minutes of the pyrolysis phase, while the peak of $\mathrm{CO}$ is a fraction of a percent higher than those of the ensuing cycles.

Neglecting these small variations, the cycles are almost indistinguishable.

It is important to note that the composition of the producer gas varies significantly as the pyrolysis phase proceeds. This behavior can be seen clearly by examining the CEM data through one ballasted gasification cycle. The fourth cycle, which is the last cycle that was followed by a complete combustion phase, was chosen to illustrate these changes and is depicted in Figure 28.

A short time delay of approximately 1.5 minutes exists from the time the pyrolysis conditions exist in the reactor until the sample gas reaches the CEM's, but it is apparent upon the switch from combustion to pyrolysis that the $\mathrm{H}_{2}$ and $\mathrm{CO}$ concentrations increase dramatically. The initial composition contains equal parts of $\mathrm{H}_{2}$ and $\mathrm{CO}$, at approximately $22 \mathrm{vol}-\%$ of the total sample gas composition, and roughly 20 vol- $\% \mathrm{CO}_{2}$. The $\mathrm{CO}$ concentration increases throughout the cycle to nearly 29 vol- $\%$, while $\mathrm{H}_{2}$ and $\mathrm{CO}_{2}$ concentrations decrease during this 


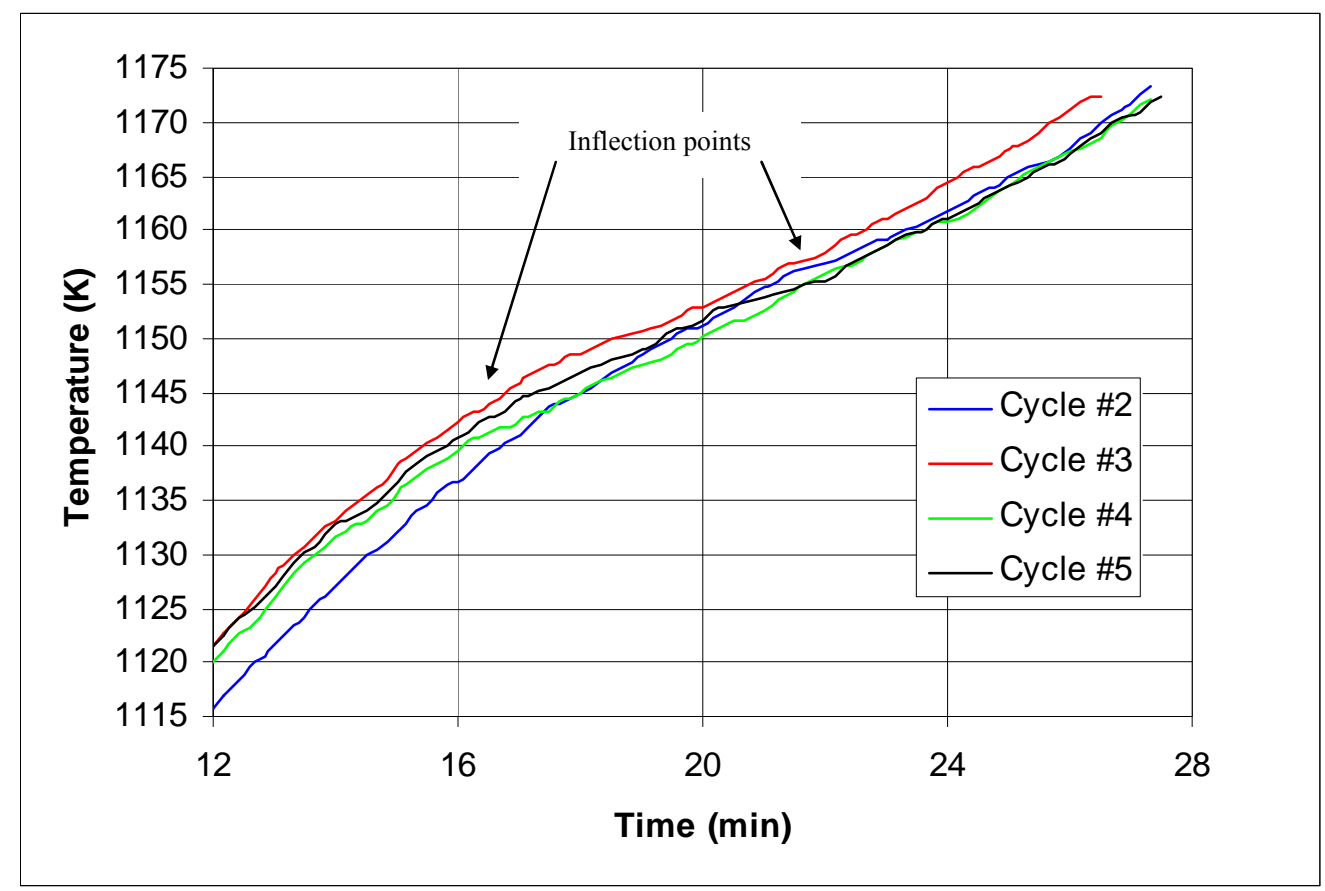

Figure 26 Bed temperature profiles illustrating phase-change during combustion 


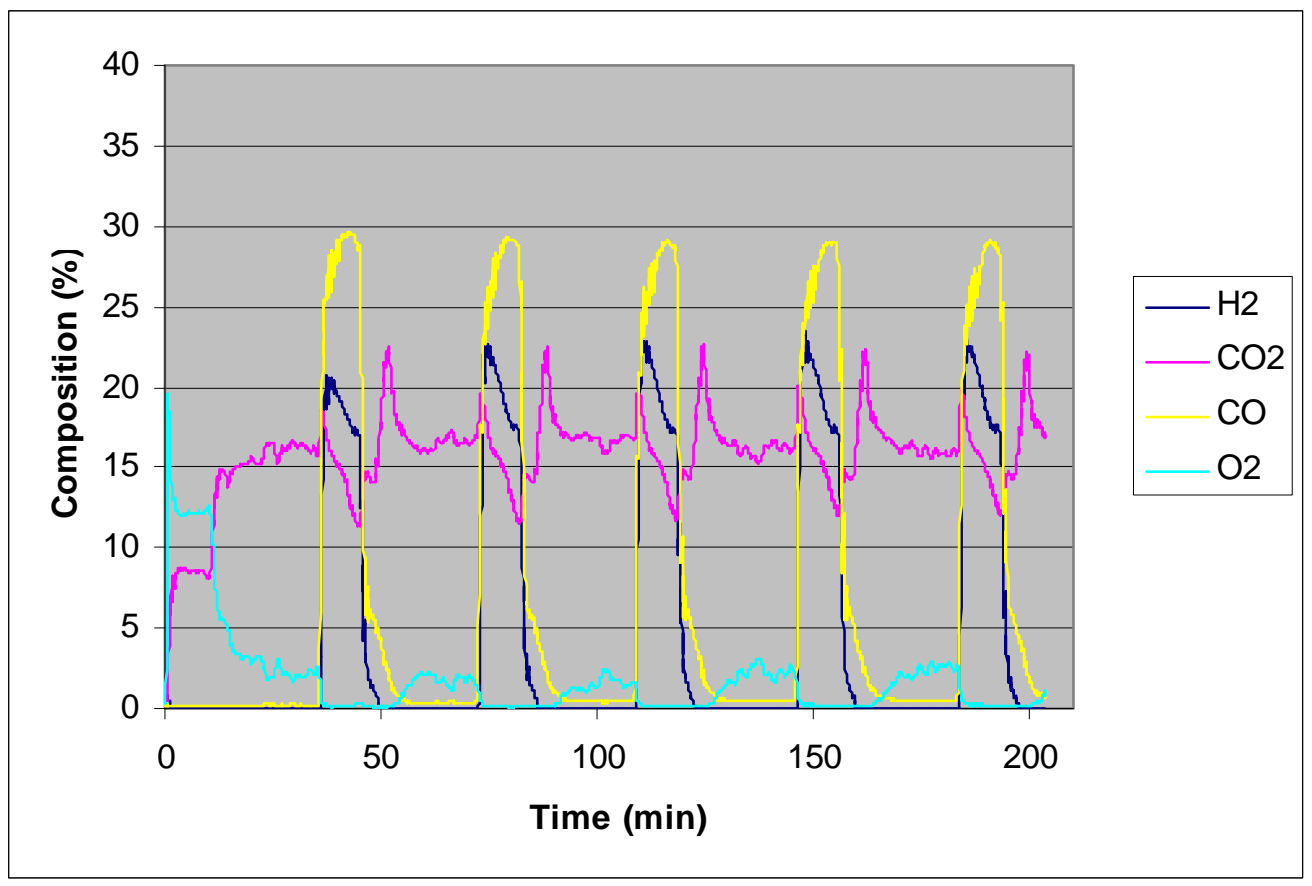

Figure 27 Composition of raw gas as determined by CEM's during pyrolysis testing

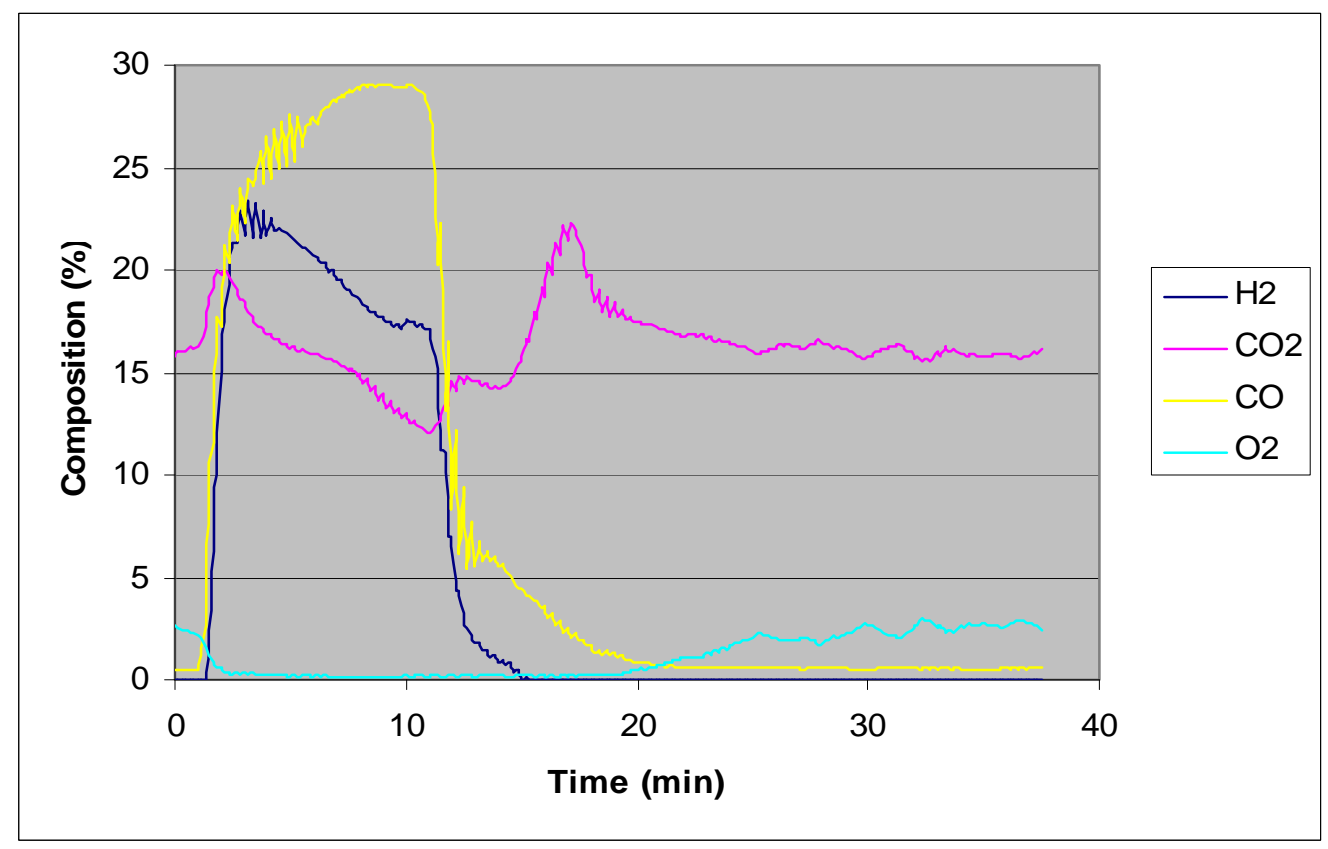

Figure 28 Composition of raw gas as determined by CEM's during cycle \#4 
time to approximately 17 vol- $\%$ and 12 vol- $\%$, respectively. As the pyrolysis phase ends and steam flow is ceased, air is reintroduced into the bed and a short period of char burnout commences. This char, produced during the pyrolysis phase, is incompletely combusted, as evidenced by the presence of a significant amount of $\mathrm{CO}$ and a small portion of $\mathrm{H}_{2}$. As char burnout comes to completion, $\mathrm{CO}_{2}$ production spikes. Following this spike, excess oxygen becomes evident and the combustion phase continues in a relatively stable fashion.

While only $\mathrm{H}_{2}, \mathrm{CO}$ and $\mathrm{CO}_{2}$ are monitored continuously during the pyrolysis, the micro-GC data from several cycles can be manipulated to provide profiles of the other species present in the gas. Due to the time required by the micro-GC to draw a sample and analyze the gas, only three to four measurements could be obtained during the pyrolysis phase of the cycle. However, the intermittent micro-GC data can be superimposed upon the CEM data, which is done in Figure 29. Furthermore, the consistency of the CEM data from cycle to cycle allows the construction of a composite graph illustrating the trend of micro-GC data throughout the cycle, shown in Figure 30 .

From the composite micro-GC data, the patterns of the $\mathrm{H}_{2}, \mathrm{CO}$ and $\mathrm{CO}_{2}$ are consistent with the CEM data. This provides evidence that the composite gas composition is accurate throughout the cycle and instills confidence in the measurements of gases not included in the CEM data: nitrogen $\left(\mathrm{N}_{2}\right)$; methane $\left(\mathrm{CH}_{4}\right)$; acetylene $\left(\mathrm{C}_{2} \mathrm{H}_{2}\right)$, and ethylene $\left(\mathrm{C}_{2} \mathrm{H}_{4}\right)$, which are measured together; ethane $\left(\mathrm{C}_{2} \mathrm{H}_{6}\right)$ and propane $\left(\mathrm{C}_{3} \mathrm{H}_{8}\right)$. Concentrations of $\mathrm{CH}_{4}$ and the combination of $\mathrm{C}_{2} \mathrm{H}_{2}$ and $\mathrm{C}_{2} \mathrm{H}_{4}$ during the pyrolysis phase are approximately $10-12$ vol- $\%$ and $4-5$ vol- $\%$, respectively, while $\mathrm{N}_{2}$ concentrations range from $18-22 \mathrm{vol} \%$.

The $\mathrm{N}_{2}$ present in the producer gas does not arise from the pyrolysis of biomass. Fuel-borne nitrogen comprises a very small portion of the biomass fuel fed to the reactor, typically on the order of $1 \%$ by weight, or $0.6 \%$ on a molar basis for the switchgrass and corn used in for these experiments. Thus, only a negligible amount of $\mathrm{N}_{2}$ exists in the products of pyrolysis, and all $\mathrm{N}_{2}$ present in the products of the steam pyrolysis process is assumed to originate from purge gas that passes through the reactor. This nitrogen is an undesirable product of the process and only serves to prevent the off-gassing of biomass within the feeding system prior to its insertion into the reactor. In principle, the feeding system could be designed to eliminate the need of purge gas, or recycled producer gas could be used in this role. This would increase the heating value and hydrogen content of the gas. The current gas data was adjusted to remove the nitrogen from the producer gas composition. A comparison of the hydrogen content and heating value of each sample point for both the raw gas composition and the adjusted $\left(0 \% \mathrm{~N}_{2}\right)$ composition is listed in Table 8, and Figure 31 illustrates the micro-GC data adjusted to $0 \% \mathrm{~N}_{2}$.

Although the hydrogen content of the producer gas decreases as temperature decreases during the pyrolysis cycle, this does not require the elevation of reactor operating temperatures to maximize hydrogen production. Catalytic cracking processes may be employed to convert hydrocarbons to additional hydrogen, and the water-gas shift reaction can be utilized to convert the abundant carbon monoxide and steam to hydrogen and carbon dioxide. Only if these processes prove inefficient or cost-prohibitive would reactor temperatures be required to increase in order to increase hydrogen content. 


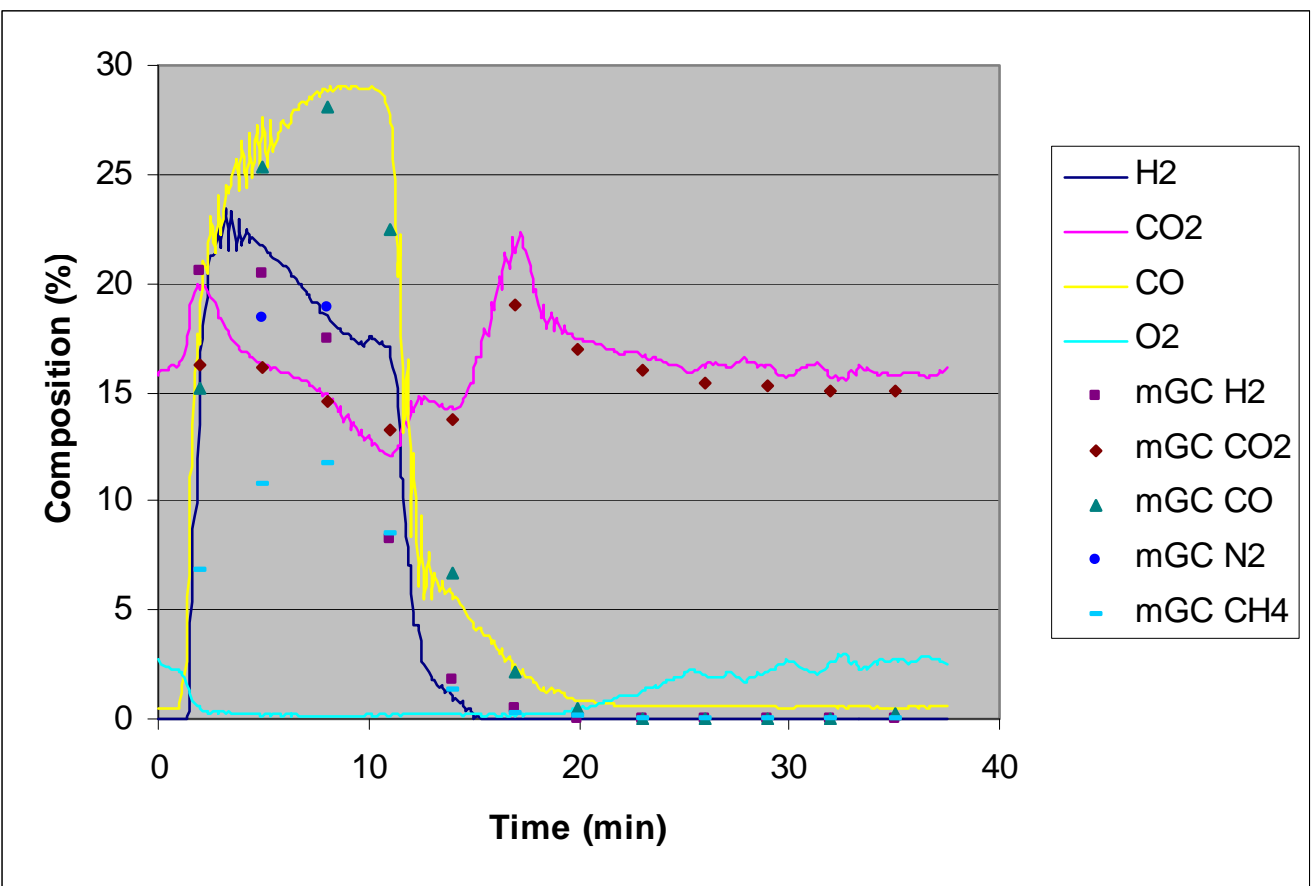

Figure 29 Cycle \#4 CEM data with selected micro-GC data superimposed

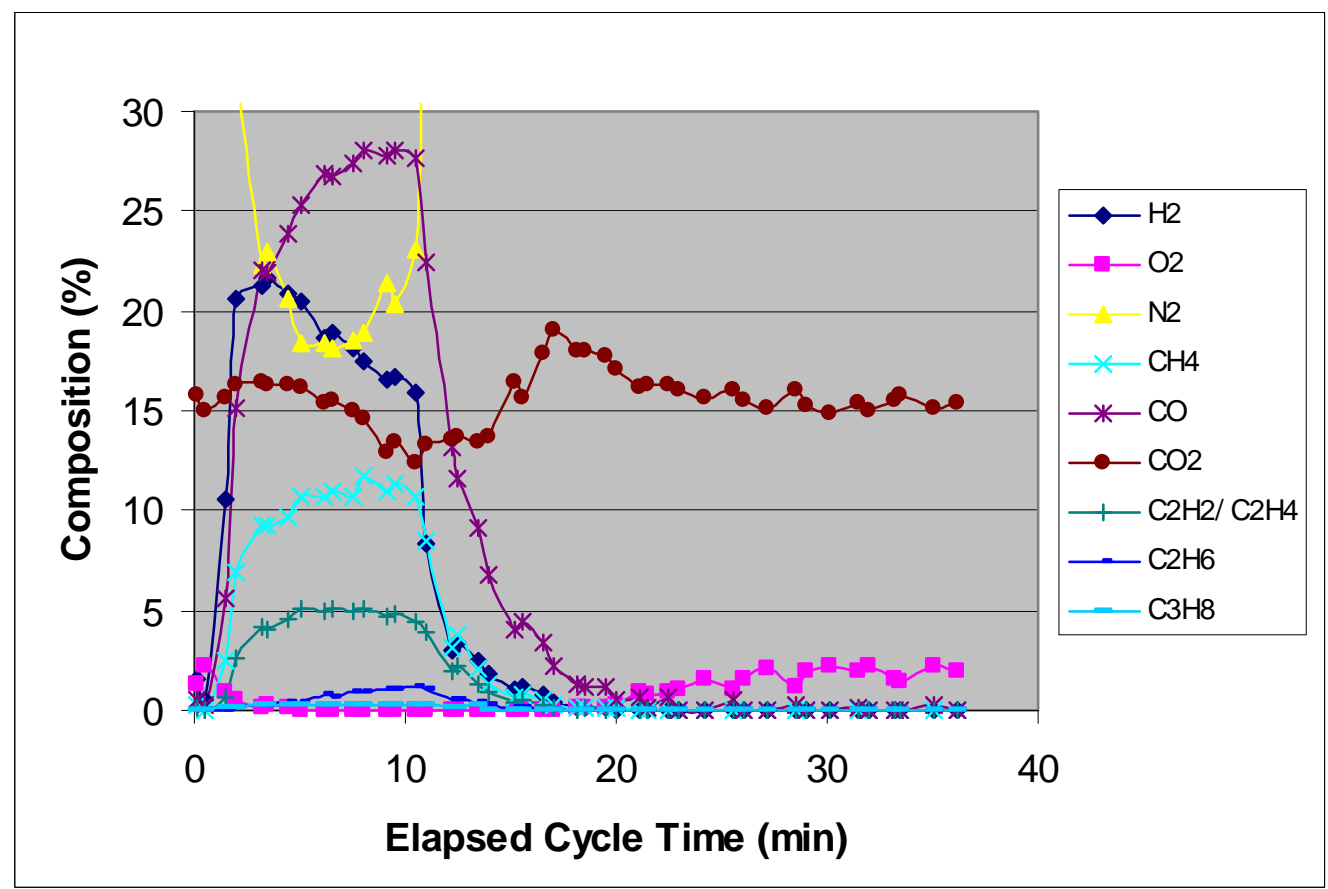

Figure 30 Composite of micro-GC data during pyrolysis testing 
Table 8 Comparison of gas qualities for raw gas and gas adjusted to $0 \% \mathrm{~N}_{2}$

\begin{tabular}{llrll}
\hline $\begin{array}{l}\text { Cycle Time } \\
\text { (min) }\end{array}$ & \multicolumn{2}{l}{ Raw composition } & \multicolumn{2}{l}{ Adjusted composition $\left(0 \% \mathrm{~N}_{2}\right)$} \\
\hline 2.00 & $\mathrm{H}_{2}$ content $(\%)$ & $\mathrm{HHV}\left(\mathrm{kJ} / \mathrm{Nm}^{3}\right)$ & $\mathrm{H}_{2}$ content $(\%)$ & $\mathrm{HHV}\left(\mathrm{kJ} / \mathrm{Nm}^{3}\right)$ \\
3.17 & 20.61 & 8,600 & 31.18 & 13,000 \\
3.50 & 21.27 & 11,500 & 27.39 & 14,800 \\
4.50 & 21.61 & 11,500 & 28.04 & 14,900 \\
5.00 & 20.87 & 12,200 & 26.27 & 15,300 \\
6.17 & 20.46 & 13,000 & 25.06 & 15,900 \\
6.50 & 18.71 & 13,100 & 22.92 & 16,100 \\
7.50 & 18.96 & 13,300 & 23.17 & 16,200 \\
8.00 & 18.11 & 13,200 & 22.24 & 16,200 \\
9.17 & 17.44 & 13,700 & 21.50 & 16,800 \\
9.50 & 16.54 & 13,100 & 21.03 & 16,700 \\
10.50 & 16.73 & 13,400 & 21.00 & 16,800 \\
\hline
\end{tabular}

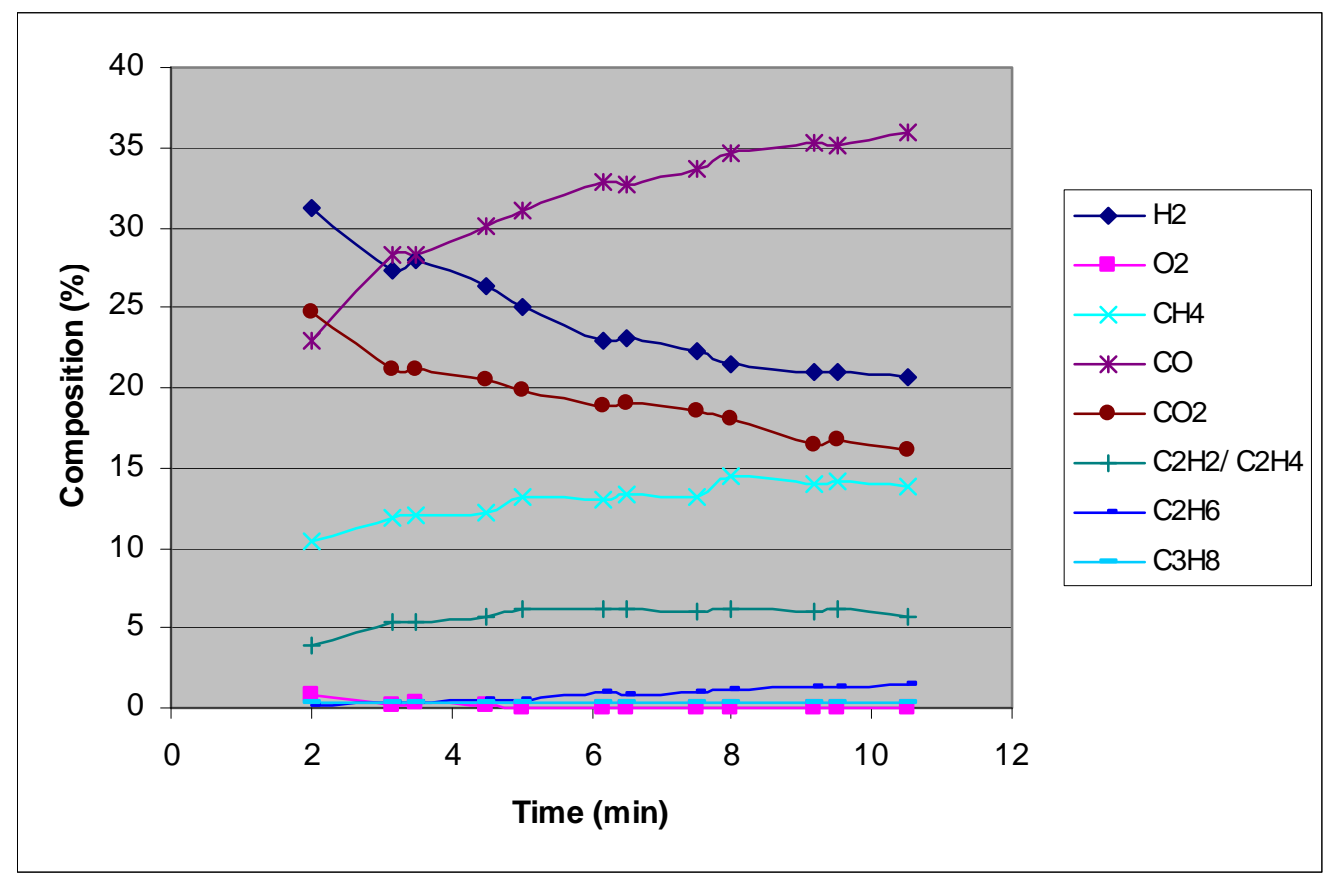

Figure 31 Composition of Micro-GC data during pyrolysis phase (adjusted to $0 \% \mathrm{~N}_{2}$ ) 


\section{$\underline{\text { System efficiency considerations }}$}

As mentioned previously, the complete ballasted cycle, i.e. both the pyrolysis phase and the combustion phase, must be evaluated to determine the efficiency of the process. One means of quantifying the efficiency at which the system converts biomass into useful energy is to determine the ratio of the amount of biomass pyrolyzed to the total biomass fed to the reactor. Table 9 and Table 10 contain data listing cycle times and fuel feed rates. The pyrolysis phase lasts 10.0 minutes at a biomass feed rate of $182 \mathrm{~kg} / \mathrm{hr}$ and the combustion phase requires 27.0 minutes at a biomass feed rate of $68 \mathrm{~kg} / \mathrm{hr}$ to obtain the prescribed temperature. Adjusting the total fuel fed for each phase of the cycle to a one-hour time period suggests that approximately $49 \%$ of the fuel is pyrolyzed.

There are several methods to improve the pyrolyzed fuel conversion efficiency. Methods include reduction of heat loss from the reactor, implementation of a char burn-out period prior to feeding biomass during the combustion cycle, preheat the combustion air, and reduce the amount of purge gas used during pyrolysis. Each of these will be discussed in more detail.

Minimization of heat loss from the system will improve pyrolysis of the biomass and/or increase the length of the pyrolysis cycle. A sensitivity analysis using the model supports this hypothesis. Further analysis of the heat transfer to and from the refractory suggests the thermal mass of the wall plays a significant role in energy storage/release when cycling between combustion and pyrolysis phases. An analysis of thermal mass of the walls of the reactors is shown in Table 11. From this comparison, it can be seen that an additional $172 \mathrm{~kJ} / \mathrm{K}$ is stored in the wall of the reactor as the refractory is heated during the combustion phase. While this additional thermal capacity would not affect a steady-state process to a significant degree, it is significant for the dynamic ballasted gasification system. It is hypothesized that large quantities of thermal energy are transferred to and from the refractory during the combustion and pyrolysis phases. If the average temperature within the refractory fluctuated by $150 \mathrm{~K}$ during the operation of the cycle (the fluidized bed temperature fluctuates $250 \mathrm{~K}$ during this time), an additional $25.8 \mathrm{MJ}$ would be stored within the refractory. There are currently no means of measuring the temperature of the refractory in the reactor, and so a precise determination of the role this large thermal mass plays in the operation of the reactor cannot be quantified.

The char burnout period is critical to cycle behavior as it accomplishes two important tasks. First, it removes the char that accumulates within the bed during pyrolysis, and second, the oxidation of the char provides thermal energy to the bed. In this study, air and biomass were fed to the reactor immediately following the conclusion of the pyrolysis phase. Because of the char present during the portion of this combustion phase, there was not enough air to combust all of the fuel within the reactor, and so the exhaust stream contained products of incomplete combustion. This can be seen in the graph of the chemical composition of Cycle 4 (see Figure 29). Significant amounts of carbon monoxide and hydrogen are present in the producer gas, and these products carry a certain amount of chemical exergy with them as they leave the reactor. In effect, the removal of this chemical exergy robs the fluidized bed of thermal energy, increasing the amount of biomass that must be combusted to raise the temperature of the bed.

The inlet temperature of steam has been found to be a significant factor in determining reactor behavior during pyrolysis, and it is reasonable to believe that inlet temperature of the air has a 
Table 9 Ballasted gasification cycle times

\begin{tabular}{|c|c|c|c|c|}
\hline \multicolumn{5}{|c|}{ Process time (min) } \\
\hline $\begin{array}{l}\text { Char } \\
\text { comb. }\end{array}$ & $\begin{array}{c}\text { Biomass } \\
\text { comb. }\end{array}$ & $\begin{array}{l}\text { Total } \\
\text { comb. }\end{array}$ & Pyrolysis & $\begin{array}{c}\text { Cycle } \\
\text { total }\end{array}$ \\
\hline- & 27.0 & 27.0 & 10.0 & 37.0 \\
\hline
\end{tabular}

Table 10 Fraction of fuel pyrolyzed

\begin{tabular}{cccccc}
\hline $\begin{array}{c}\text { Combustion } \\
\text { fuel flow } \\
\text { rate }(\mathrm{kg} / \mathrm{hr})\end{array}$ & $\begin{array}{c}\text { Pyrolysis } \\
\text { fuel flow } \\
\text { rate }(\mathrm{kg} / \mathrm{hr})\end{array}$ & $\begin{array}{c}\text { Cycles per } \\
\text { hour }\end{array}$ & $\begin{array}{c}\text { Total fuel } \\
\text { combusted } \\
(\mathrm{kg} / \mathrm{hr})\end{array}$ & $\begin{array}{c}\text { Total fuel } \\
\text { pyrolyzed } \\
(\mathrm{kg} / \mathrm{hr})\end{array}$ & $\begin{array}{c}\text { Fuel fraction } \\
\text { pyrolyzed }\end{array}$ \\
\hline 68 & 182 & 1.6 & 49.7 & 48.5 & $49 \%$ \\
\hline
\end{tabular}

Table 11 Thermal mass of the refractory

\begin{tabular}{ccccc}
\hline & \multicolumn{2}{c}{ Refractory } & & Total \\
thickness & $\mathrm{m}$ & $\mathrm{c}_{\mathrm{p}}$ & $\mathrm{m}^{*} \mathrm{c}_{\mathrm{p}}$ & $\mathrm{m}^{*} \mathrm{c}_{\mathrm{p}}$ \\
$(\mathrm{cm})$ & $(\mathrm{kg})$ & $(\mathrm{kJ} / \mathrm{kg}-\mathrm{K})$ & $(\mathrm{kJ} / \mathrm{K})$ & $(\mathrm{kJ} / \mathrm{K})$ \\
\hline 7.62 & 187.6 & 0.92 & 172.6 & 172.6 \\
\hline
\end{tabular}


similar effect during the combustion phase. This study did not employ preheating of the combustion air, and the inlet temperature profiles are plotted with bed temperature profiles for both steam cooling and pyrolysis tests in Figure 32 and Figure 33, respectively. These graphs illustrate that the inlet temperature is below $400 \mathrm{~K}$ throughout the majority of the combustion phase. If stoichiometric combustion is considered, and the biomass is assumed to have the molar formula of $\mathrm{CH}_{2} \mathrm{O}$, the combustion equation can be written as:

Eq. 14

$$
\mathrm{CH}_{2} \mathrm{O}+\left(\mathrm{O}_{2}+3.76 \cdot \mathrm{N}_{2}\right) \rightarrow \mathrm{CO}_{2}+\mathrm{H}_{2} \mathrm{O}+3.76 \cdot \mathrm{N}_{2}
$$

According to a proximate analysis conducted for seed corn, the higher heating value, $H H V$, has a value of $17,300 \mathrm{~kJ} / \mathrm{kg}$. This value is defined based on the enthalpies of formation, but does not consider the sensible enthalpy of the reactants and products. If these sensible enthalpies are considered, the enthalpy of the combustion reaction, $\Delta h_{\text {comb }}$, can be calculated and is considerably lower than the higher heating value, $H H V$, as a large amount of sensible energy leaves the reactor with the products of combustion. Rather than the higher heating value, it is the enthalpy of combustion that best describes the amount of energy released to raise the temperature of the fluidized bed. By examining the enthalpy of combustion for air inlet temperatures of 400 $\mathrm{K}$ and $720 \mathrm{~K}$, it is clear that raising the inlet temperature increases the amount of energy released during the combustion process. It is assumed that the products of combustion are raised to the bed temperature. Thus, as the temperature of the bed increases, the enthalpy of combustion decreases due to the increased exit temperature of the products of combustion. A comparison of enthalpies of combustion throughout the process for two inlet air temperatures, $420 \mathrm{~K}$ and $720 \mathrm{~K}$, is shown in Table 12.

Based on the results of a sensitivity analysis of the purge gas flow rate, this gas can also be a significant heat sink during the operation of the reactor. It is unclear, however, how considerable this sink is during the combustion process, when large amounts of thermal energy are introduced into the reactor.

There appear to be several factors that result in the lengthy combustion period observed in these experiments. One of the most significant factors is the large thermal mass represented by the increased refractory within the reactor, but it is unlikely that this parameter can be drastically altered. Therefore, improvements to bed operation must focus on changes to parameters that can be effectively modified. The most effective means of shortening the combustion phase would be to preheat the air which fluidizes the bed and provides the oxidant for combustion. Conducting a char burnout phase would also better utilize the fuel present within the reactor and increase the fraction of fuel pyrolyzed. The period could be further reduced by wrapping the bed with external blanket insulation and decreasing the flow rate of the purge gas, although these would provide less substantial improvements in bed behavior than the other factors mentioned.

A more detailed analysis of the ballasted gasification cycle would be greatly facilitated by the closure of a mass balance around the system, and so an attempt was made to close such a balance. The most vital portion of this mass balance was the mass flow rates of the respective species within the producer gas. As mentioned previously, all nitrogen present in the producer gas is believed to be derived from the purge gas. Thus, it was hypothesized that the purge gas 


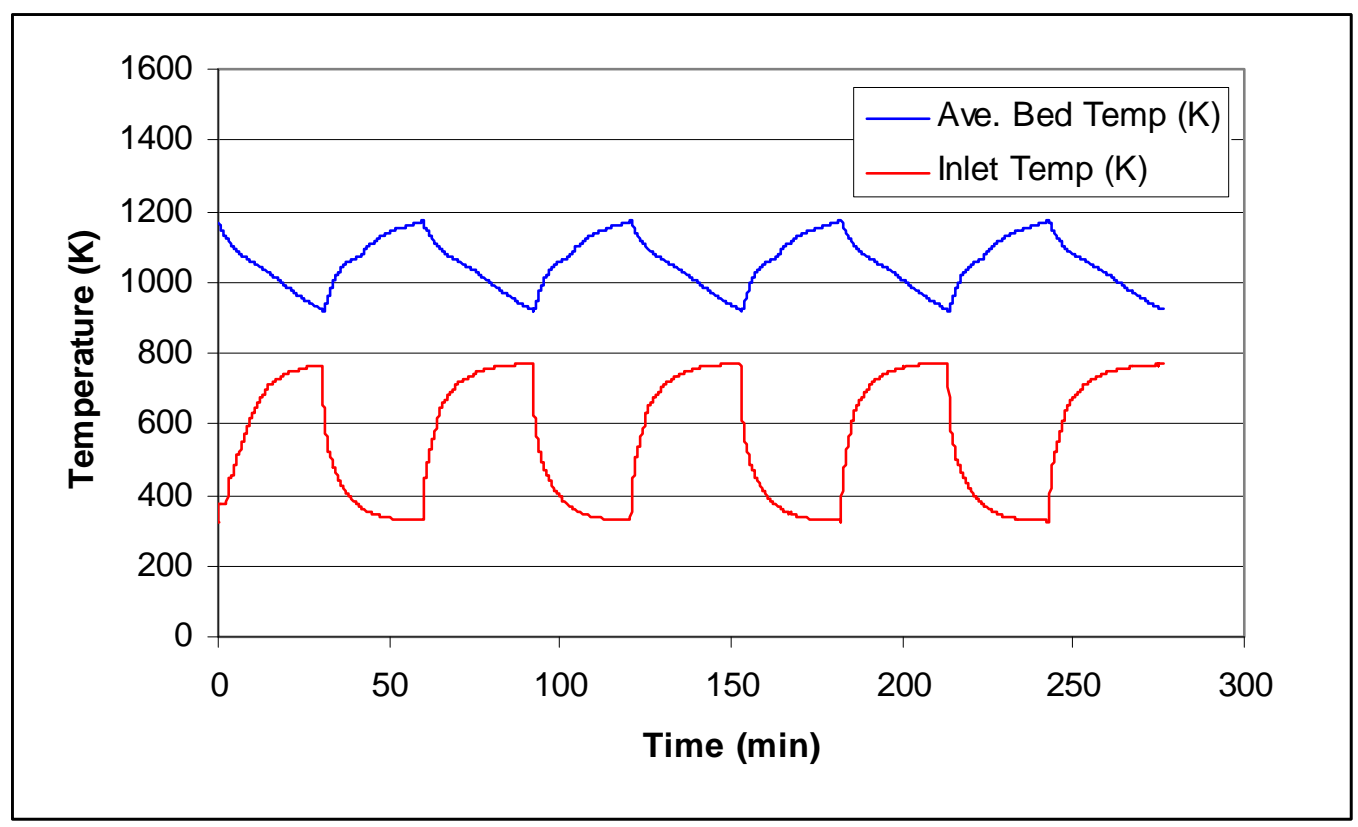

Figure 32 Average bed temperature and inlet temperature during steam cooling cycle

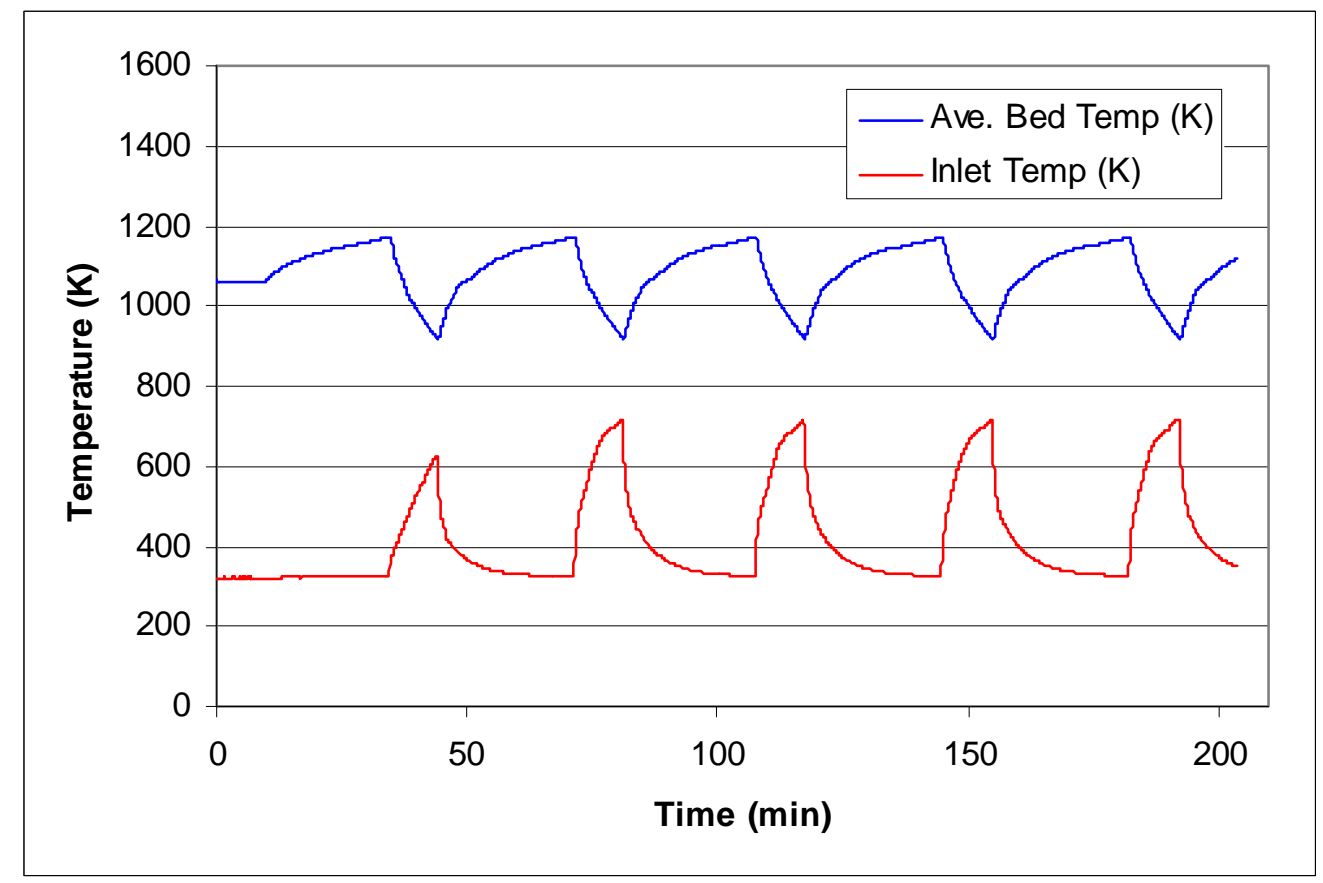

Figure 33 Average bed temperature and inlet temperature during ballasted gasification 
Table 12 A comparison of enthalpy of combustion for varying inlet air temperatures

\begin{tabular}{|l|l|l|l|}
\hline \multirow{2}{*}{ Bed Temp $(\mathrm{K})$} & \multicolumn{2}{|l|}{$\Delta h_{\text {comb }}(\mathrm{kJ} / \mathrm{kg}$ of biomass $)$} & \multirow{2}{*}{ \% Diff. } \\
\cline { 2 - 4 } & $\begin{array}{l}\mathrm{T}_{\text {air,in }}=400 \\
\mathrm{~K}\end{array}$ & $\begin{array}{l}\mathrm{T}_{\text {air,in }}=720 \\
\mathrm{~K}\end{array}$ & \\
\hline 922 & 10360 & 11900 & +14.8 \\
\hline 1000 & 10900 & 12440 & +14.1 \\
\hline 1100 & 11640 & 13180 & +13.2 \\
\hline 1172 & 12200 & 13740 & +12.6 \\
\hline
\end{tabular}

could be used as a "tracer" to determine the producer gas mass flow rates. As the micro-GC provided composition on a molar basis, the ratio the mole fraction of a given species, $x_{i}$, to the mole fraction of nitrogen $\left(\mathrm{N}_{2}\right), x_{N_{2}}$, would be equal to the ratio of the respective volumetric flowrates, $\dot{V}_{i}$ and $\dot{V}_{N_{2}}$, according to the equation:

Eq. 15

$$
\frac{x_{i}}{x_{N_{2}}}=\frac{\dot{V}_{i}}{\dot{V}_{N_{2}}}
$$

However, uncertainty arose in the nitrogen flow rate through the reactor from two sources. The first source of uncertainty was due to the omission of a pressure gauge downstream of the rotameter used to measure the flow. Due to this, the pressure at the rotameter was uncertain, and correction calculations were unable to be performed. The second source of uncertainty was due to leaks in the herbaceous fuels feeding system. The leaks from this system have not been quantified, but they are thought to be significant. Because of these uncertainties, the nitrogen flow rate through the reactor could not be confidently quantified, and thus, this method was unqualified to quantify the producer gas flow rate. A more detailed mass and energy balance analysis is provided in Section 5 of this report.

\subsection{Moving Bed Filtration}

\section{$\underline{\text { Hot Gas Experiments }}$}

The MBGF has been operated for over 150 hours on producer gas at a nominal flow rate of 340 $\mathrm{nm}^{3} \mathrm{~h}^{-1}$ at temperatures exceeding $600{ }^{\circ} \mathrm{C}$ and dust loadings on the order of $30 \mathrm{~g} \mathrm{~nm}^{-3}$ and heavy tar loading of about $20 \mathrm{~g} \mathrm{~nm}^{-3}$. The nominal gas composition of the producer gas during these tests is reported in Table 13, which is typical of air-blown gasification. The heating value associated with these numbers is $5.85 \mathrm{MJ} \mathrm{nm}^{-3}$.

Table 13 Composition of producer gas

\begin{tabular}{|c|c|c|c|c|c|c|}
\hline Constituent & $\mathrm{H}_{2}$ & $\mathrm{CO}$ & $\mathrm{CH}_{4}$ & $\mathrm{C}_{2} \mathrm{H}_{4}$ & $\mathrm{CO}_{2}$ & $\mathrm{~N}_{2}$ \\
\hline Vol-\% & 11.7 & 10.2 & 4.4 & 2.8 & 14.8 & 50.4 \\
\hline
\end{tabular}

Pressure drop across the MBGF as a function of time is illustrated in Figure 34 for two trials of the filter representing different granular media flow rates of $4.4 \mathrm{~kg} \mathrm{~h}^{-1}$ and $6.6 \mathrm{~kg} \mathrm{~h}^{-1}$. Pressure drop was in the range of $60-65 \mathrm{~mm}$ water early in the test while the gasifier was being 

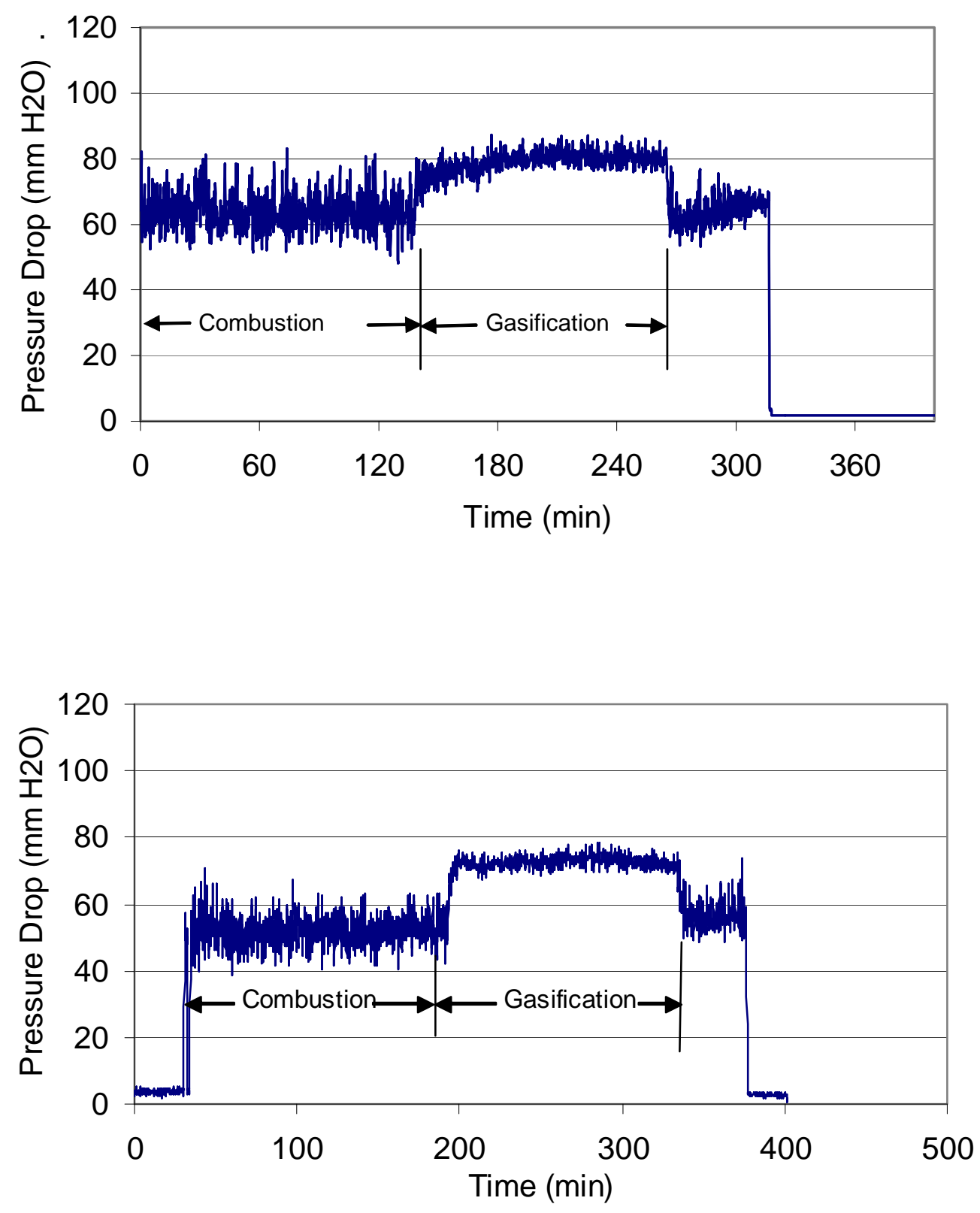

Figure 34 Pressure drop through the moving bed granular filter ( $80 \mathrm{scfm}, 650 \mathrm{C}$ ) for two granular flow rates (a) $3.3 \mathrm{~kg} / \mathrm{h}$ and (b) $6.6 \mathrm{~kg} / \mathrm{h}$. 
preheated by combustion. Once the reactor was transitioned to gasification, the pressure drop increased to the range of $75-80 \mathrm{~mm}$ water; this increase is the result of higher gas flow rate during gasification compared to combustion. Comparing the plots for the different granular media flow rates, it is evident that higher granular flow rates approach steady state more quickly and yield lower pressure drops, a result consistent with the formation of filter cake.

Problems with maintaining gas seals in the sampling lines at high temperatures and confirming the existence of isokinetic sampling conditions have limited our ability to measure particulate concentrations at the exit of the MBGF. We can only offer limited quantitative data at hot gas conditions, which is why undertook the extensive cold-flow measurements described subsequently. In some trials the filtration efficiency reached $97 \%$ although these results were not repeatable with any degree of reliability. Although these numbers are consistent with results reported in the literature for other moving bed granular filters, our own cold flow studies, subsequently described, saw evidence that char is not as readily removed by a moving bed filter as is fly ash.

Particles collected in the sampling filters were subjected to scanning electron microscopy and energy-dispersive spectroscopy to determine elemental analysis. Furthermore an ATM Model L3P Sonic Sifter was used to get size distributions of dust entering the MBGF. The average size of the inlet dust particles was $26.1 \mu \mathrm{m}$. Scanning electron micrographs of particulate collected at the inlet and exit of the filter were prepared to compare the size of particles at the two locations. Figure 35 shows that a large fraction of the particulate at the inlet are $25-30 \mu \mathrm{m}$ in diameter. At the outlet, there are few particles as large as $10 \mu \mathrm{m}$ and most appear to be in the size range of $2-3 \mu \mathrm{m}$.

Elemental analyses of the inlet and exit streams to the MBGF, illustrated in Figure 36, show no dramatic change in dust composition. Potassium decreases on the order of $25 \%$ in the exit stream, indicating that some alkali is sequestered in the coarsest particles, which are most readily captured by the MBGF. Sulfur is enriched in particles passing through the filter; the reason for this is not known. Of particular significance in the elemental analysis is the absence of silicon enrichment in the particulate at the exit, which might be expected if the silica pebbles were releasing fine dust to the gas stream as it passed through the filter. Granular media must be dustfree and non-friable to be effective in the MBGF.

\section{Cold Flow Experiments}

The results described in this subsection will be published in 2005 in the Proceedings of the Science in Thermal and Chemical Biomass Conversion Conference. ${ }^{21}$ The original configuration of the moving bed granular filter allowed evaluation of filter performance for superficial velocities in the downcomer, $U$, ranging between 0.57 and $0.95 \mathrm{~m} / \mathrm{s}$ and downcomer bed depths, $L$, ranging between 5.72 and $18.10 \mathrm{~cm}$. A semi-logarithmic plot of penetration, $P$, vs. $L / U$ is shown in Figure 37. Data in this limited $L / U$ range correlated very well with Eq. 7 and yielded extrapolated efficiencies greater than $99 \%$ (that is, $P<0.01$ ) for $L / U$ exceeding $0.5 \mathrm{~s}$.

In order to confirm the ability of the filter to achieve such high efficiencies required modification of experimental apparatus since its original configuration did not allow operation at $L / U$ greater 

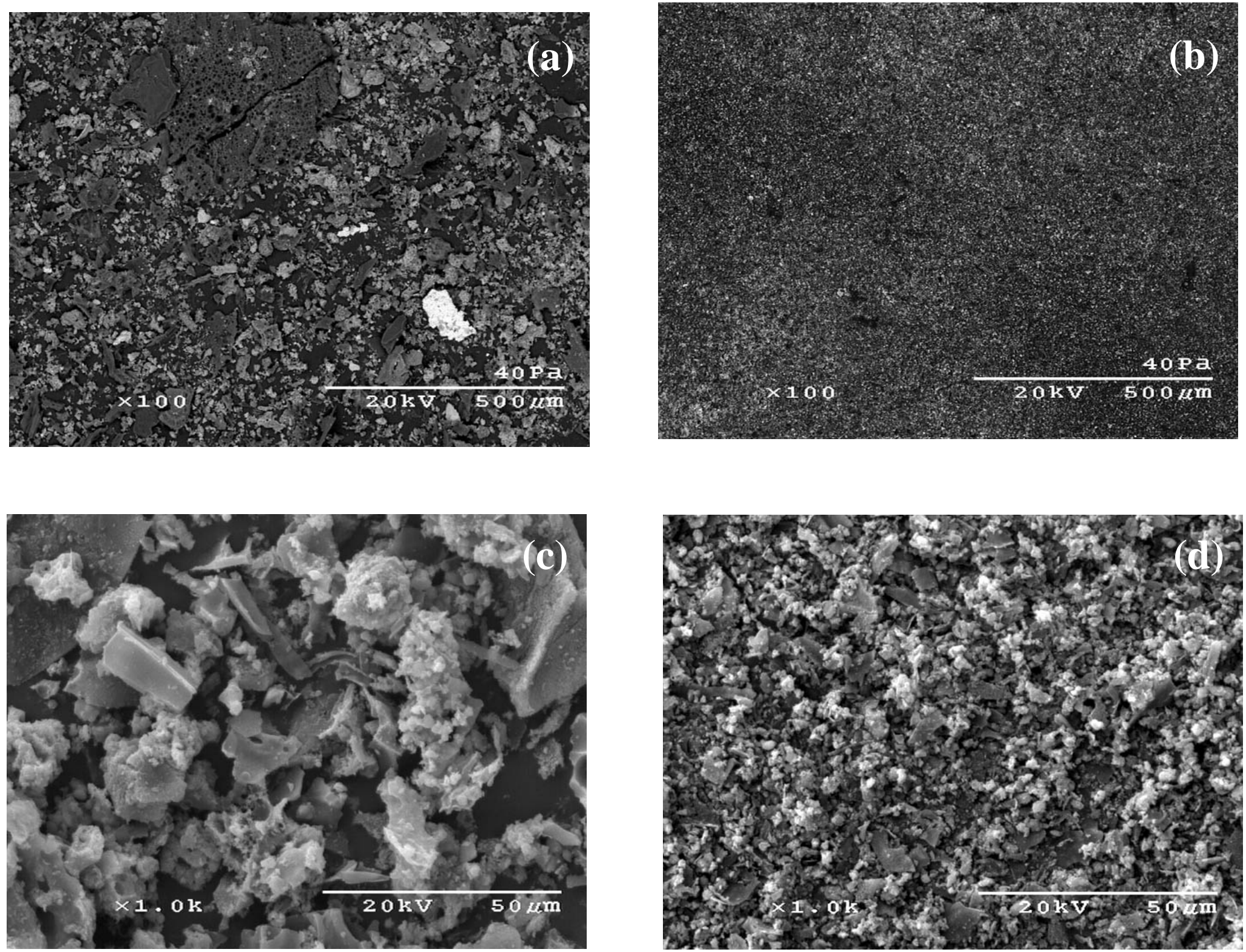

Figure 35 Scanning electron microgaphs of isokinetically sampled dust from producer gas. (a) Upstream of the MBGF at 100x magnification, (b) Downstream of the MBGF at 100x magnification, (c) Upstream of the MBGF at 1000x magnification, (d) Downstream of the MBGF at 1000x magnification. 


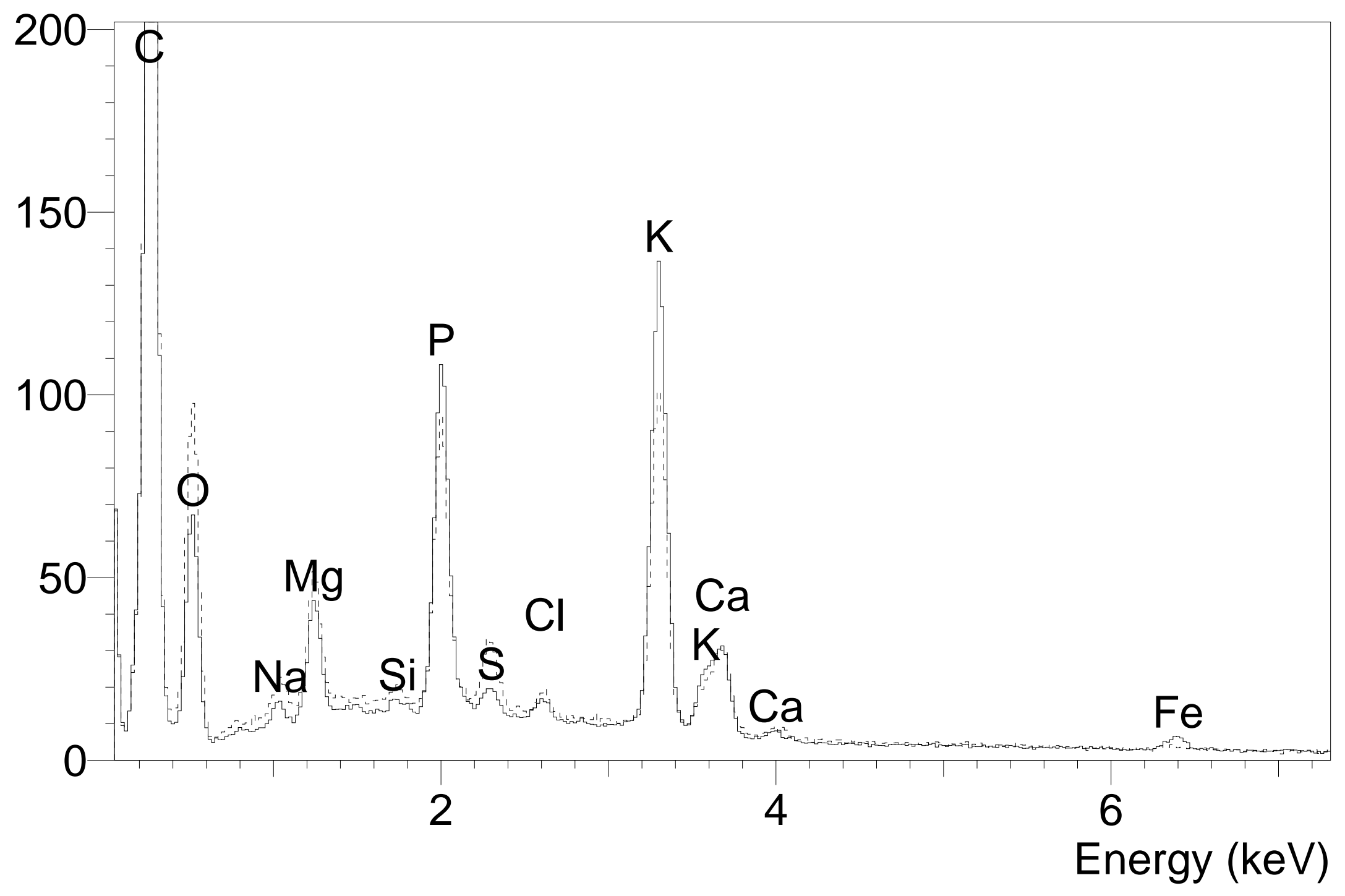

Figure 36 Elemental composition of dust in producer gas (solid line - inlet stream; dashed line - exit stream) 


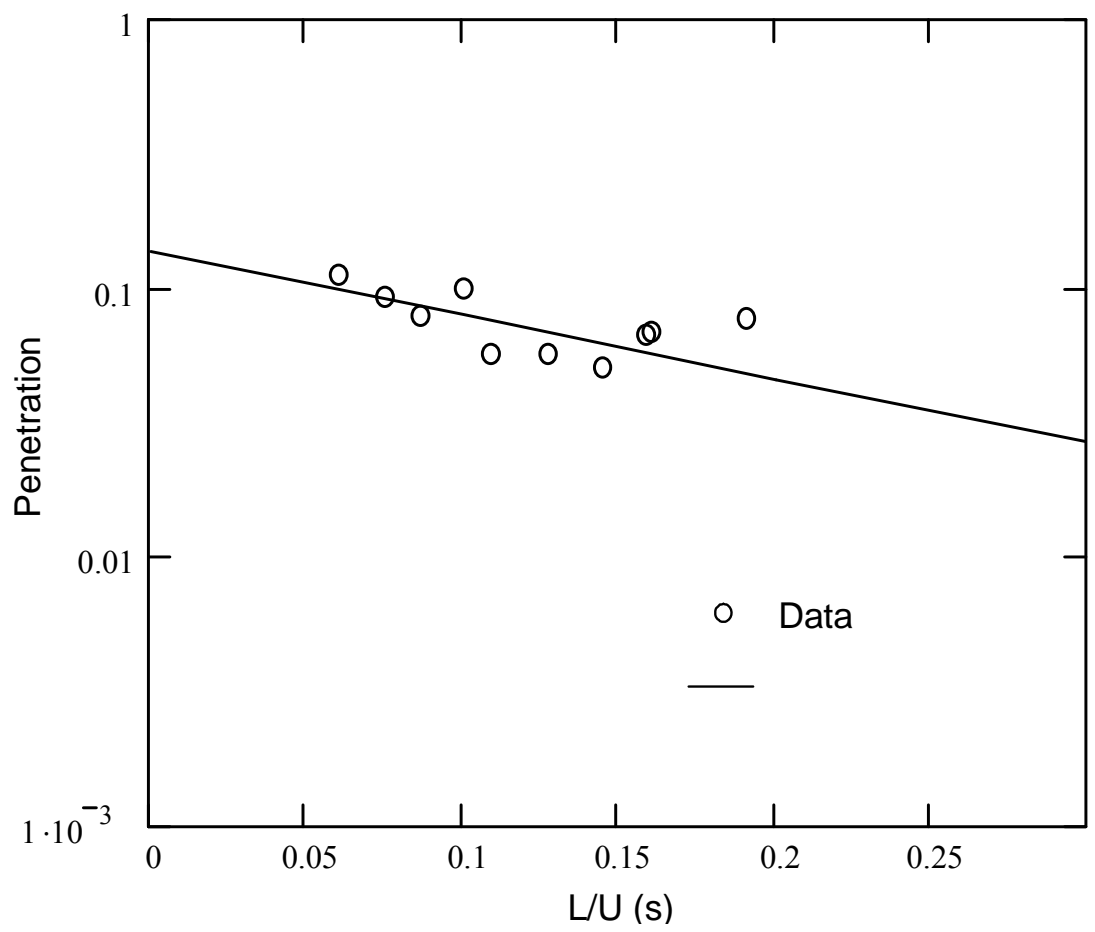

Figure 37 Analysis of penetration data using monodisperse model $(L / U<0.2)$

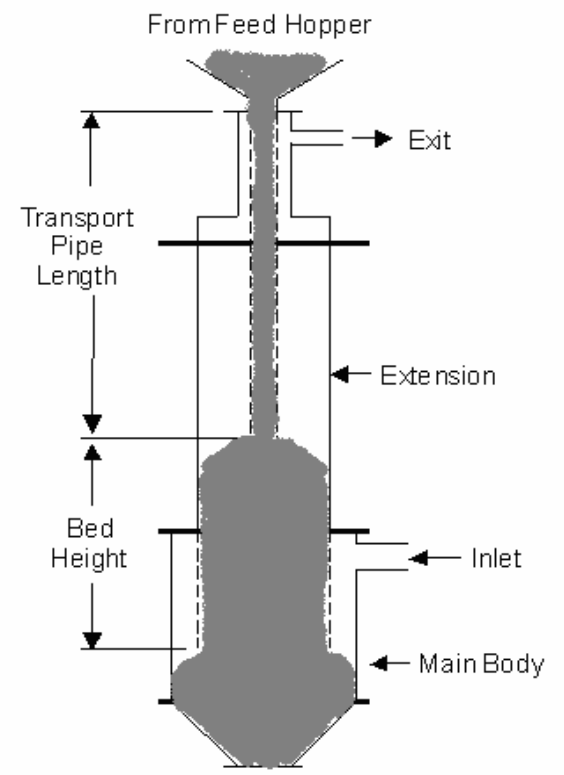

Figure 38 Modification to moving bed filter to allow evaluation of $\mathrm{L} / \mathrm{U}>0.2$ 
than $0.2 \mathrm{~s}$. To this end an extension was added to the downcomer, illustrated in Figure 38, which allowed the bed depth to range from 5 to $80 \mathrm{~cm}$ by substituting transport pipes of various lengths. In combination with gas flow rates ranging between 425 and $700 \mathrm{~L} / \mathrm{min}$, this change allowed $L / U$ to be as large as $1.1 \mathrm{~s}$.

The results of testing in this expanded range are shown in Figure 39. At the highest values of $L / U$, the correlation obtained from Eq. 7 of Section 2.2 and the data of Figure 37 greatly underestimates the penetration of dust through the filter. For example, at $L / U$ equal to $0.5 \mathrm{~s}$ the efficiency was only $97 \%$ instead of the $99 \%$ extrapolated from the application of Eq. 7 of Section 2.2. A possible explanation for this discrepancy is the biomodal distribution of the fly ash particles evident in Figure 9 in Section 2.2: particles tend to populate the size intervals around 8 $\mu \mathrm{m}$ and $85 \mu \mathrm{m}$. Particles with disperse size distributions and filtration efficiencies that are dependent on particle size are expected to show the non-ideal behavior of Figure $39^{22}$. The least penetrating particles are quickly captured in the bed with the result that the remaining particles are relatively more penetrating ${ }^{22}$. This results in semi-logarithmic plots of $P$ vs. $L$ with upward concavity.

Brown describes a model to account for poly-disperse distributions that consists of a summation of single layer penetration relationships for each particle size in the distribution ${ }^{22}$. For the fly ash distribution shown in Figure 9 in Section 2.2, a model assuming only two particle sizes ( $8 \mu \mathrm{m}$ and $85 \mu \mathrm{m})$ is a reasonable approximation and the bimodal model takes the form:

Eq. 16

$$
P=(1-f) \exp \left(-k_{1} L / U\right)+f \exp \left(-k_{2} L / U\right)
$$

The parameters $k_{1}$ and $k_{2}$ are the collection rate constants for particles of diameters $\mathrm{d}_{1}$ and $\mathrm{d}_{2}$, respectively, and (1-f) and $f$ are the fractions of particles of size $d_{1}$ and $d_{2}$, respectively. Based on Figure 9 in Section 2.2, the mass fractions for the two particle sizes are each taken to be 0.5. The bimodally dispersed model shown in

Figure 39 is the result of non-linear regression analysis of the experimental data shown in the figure. This analysis yielded values of $k_{1}$ and $k_{2}$ equal to 9.904 and 2.044, respectively, indicating distinctly different filtration efficiencies for the two particle sizes. The model, however, does not indicate whether it is the larger or smaller particles in the bimodal distribution that is filtering most efficiently.

Conventional wisdom holds that the smallest particles $(8 \mu \mathrm{m})$ would be filtered least efficiently. To investigate this possibility, filtration efficiency as a function of particle size was calculated by screening the dust collected at the exit of the filter and applying Eq. 3 in Section 2.2 to each size interval. As shown in Figure 9, the smallest particles $(3-18 \mu \mathrm{m})$ show the highest filtration efficiency, followed by the largest particles $(181-300 \mu \mathrm{m})$ while particles of the middle size (33 $-128 \mu \mathrm{m}$ ) showed the lowest filtration efficiencies - as low as $60 \%$. Since large particles contribute insignificantly to the total mass of fly ash, it is concluded for the bimodal model of Eq. 8 that $d_{1}$ represents the efficiently filtered small particles $(3-18 \mu \mathrm{m})$ and $\mathrm{d}_{2}$ represents the inefficiently filtered middle size particles $(33-128 \mu \mathrm{m})$. It is hypothesized that large particles are efficiently removed by the cyclonic flow of gases in the filter shell while the smallest particles are efficiently trapped in the granular bed. Middle size particles very likely suffer from 


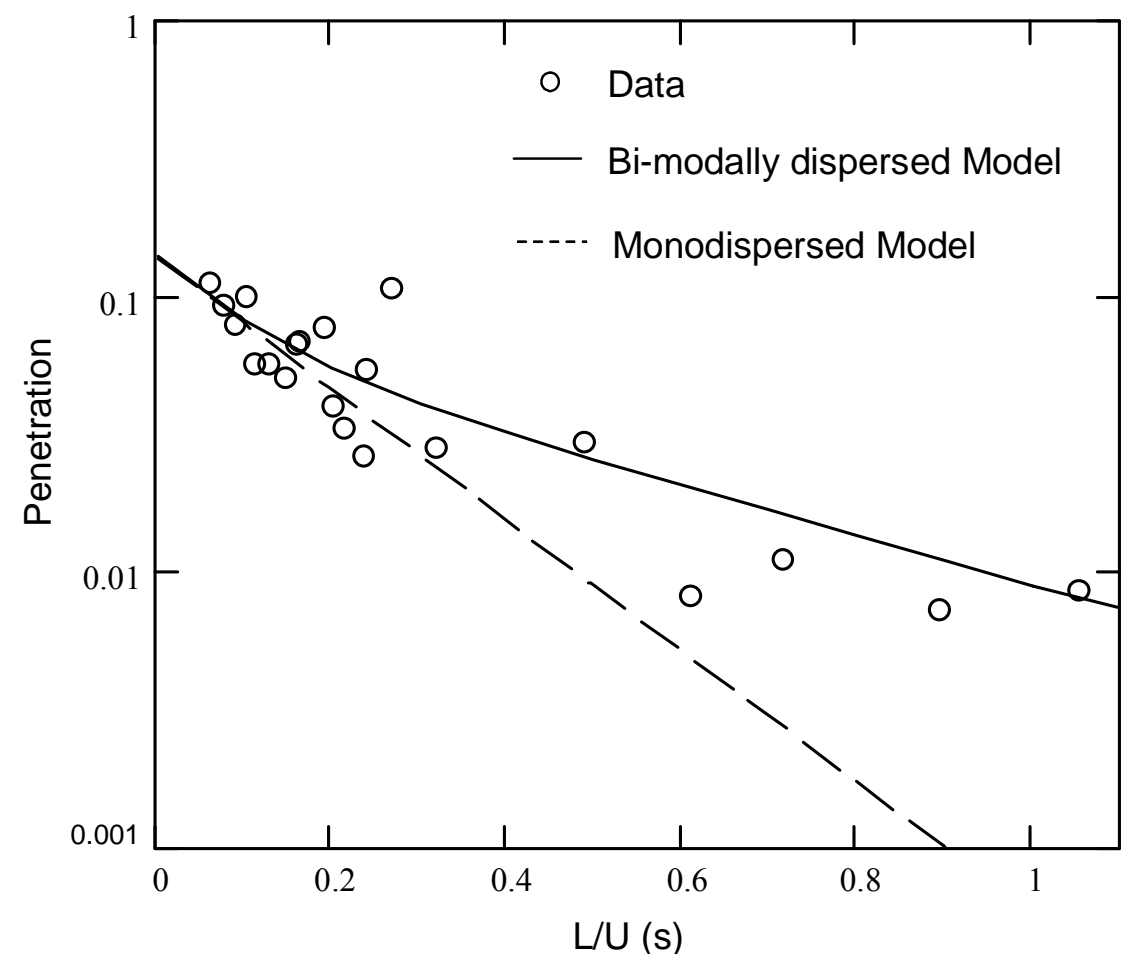

Figure 39 Analysis of penetration data using biomodally dispersed model

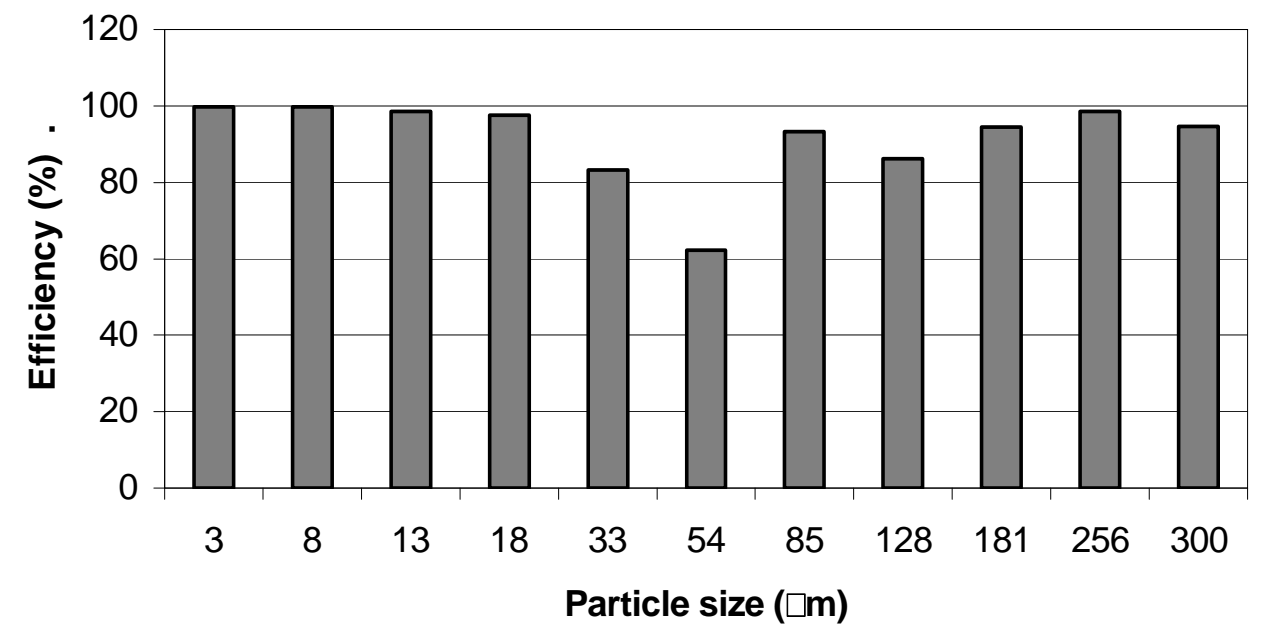

Figure 40 Filtration efficiencies for fly ash particles as a function of particle size 
both poor removal efficiency by cyclonic action and frequent rebound and re-entrainment effects in the granular bed.

Figure 39 illustrates another important observation regarding the performance of the moving bed granular filter. From Eq. 7 in Section 2.2 we expect unity penetration for L/U equal to zero: that is, all dust particles should penetrate a filter of zero depth. However, extrapolating the curves for either the mondisperse model (Eq. 7 in Section 2.2) or bimodally dispersed model (Eq. 8 in Section 2.2) back to $L / U$ equal to zero yields a penetration much less than unity. This result indicates that the interfacial zone of the filter, where dusty gas first contacts granules, is significantly filtering particles from the gas flow. Indeed, it appears that in excess of $86 \%$ of the particles are removed in the interfacial region while less than $14 \%$ of the particles are removed in the downcomer section. This filtration phenomenon at the interfacial region was also previously noted by Thambimuthu et al. ${ }^{23}$ in their study of a static granular bed filter. They formulated a model similar to Eq. 7 that includes a correction factor to account for the phenomena. The concentration, $\mathrm{C}$, at the exit of their filter was given by:

Eq. 17

$$
C=\left(1-\delta_{0}\right) C_{0} \exp \left(\frac{-K_{M} m}{A U}\right)
$$

The variable $C_{o}$ is inlet concentration, $m$ is the bed mass, $K_{M}$ is the collection rate constant per unit bed mass, $A$ is the cross-sectional area of the bed, and $U$ is the superficial velocity in the bed. The term $\left(1-\eta_{0}\right)$ corrects for what they call "surface filtration." This behavior gives strong evidence for the contention that a dust cake formed in this interfacial region is important to effective operation of the filter. Although we did not make direct observations of the interfacial region, other researchers have visually observed the dust layer and attribute high filter efficiencies due to the build up of such a layer ${ }^{24,25,26}$

We also examined the effect of dust properties on filtration efficiency. Properties of shape, density, hardness, surface roughness and particle size are important, although it is very difficult to isolate a single effect in experiments ${ }^{23}$. Table 14 compares the average particle diameter, particle density, and filtration efficiency for four dusts tested in the moving bed granular filter at an $L / U$ value of $0.23 \mathrm{~s}$ : coal fly ash, limestone, cornstarch, and oak char. Particle shape, which influences hydrodynamic behavior of the particles, is hard to characterize by a single parameter. For this purpose, scanning electron micrographs of the coal fly ash, limestone, and oak char are provided in Figure 41, Figure 42, and Figure 43 (cornstarch closely resembles the limestone in size and shape).

The densities and average particle sizes of the fly ash and limestone are similar, yet the fly ash is captured at significantly higher efficiency than the limestone (97.32\% vs. 94.67\%). The difference appears to arise primarily from the more spherical shape of the fly ash compared to the limestone, which is evident in comparing Figure 41 and Figure 42. This conclusion is in agreement with Shimada et al. ${ }^{27}$ who note that smooth, spherical particles have large adhesion forces due to a reduction in separation distances between the surfaces. 
Table 14 Properties and filtration efficiencies for several kinds of particles

\begin{tabular}{|c|r|r|r|r|}
\hline & $\begin{array}{c}\text { Coal Fly } \\
\text { Ash }\end{array}$ & Limestone & Cornstarch & Oak Char \\
\hline $\begin{array}{c}\text { Average } \\
\text { Particle Size } \\
(\mu \mathrm{m})\end{array}$ & 14 & 10 & 10 & 13 \\
\hline $\begin{array}{c}\text { Particle } \\
\text { Density* } \\
\left(\mathrm{kg} / \mathrm{m}^{3}\right)\end{array}$ & 2600 & 2700 & 1500 & 560 \\
\hline $\begin{array}{c}\text { Filtration } \\
\text { Efficiency (\%) }\end{array}$ & 97.3 & 94.7 & 91.2 & 66.2 \\
\hline
\end{tabular}

* Reference 28 


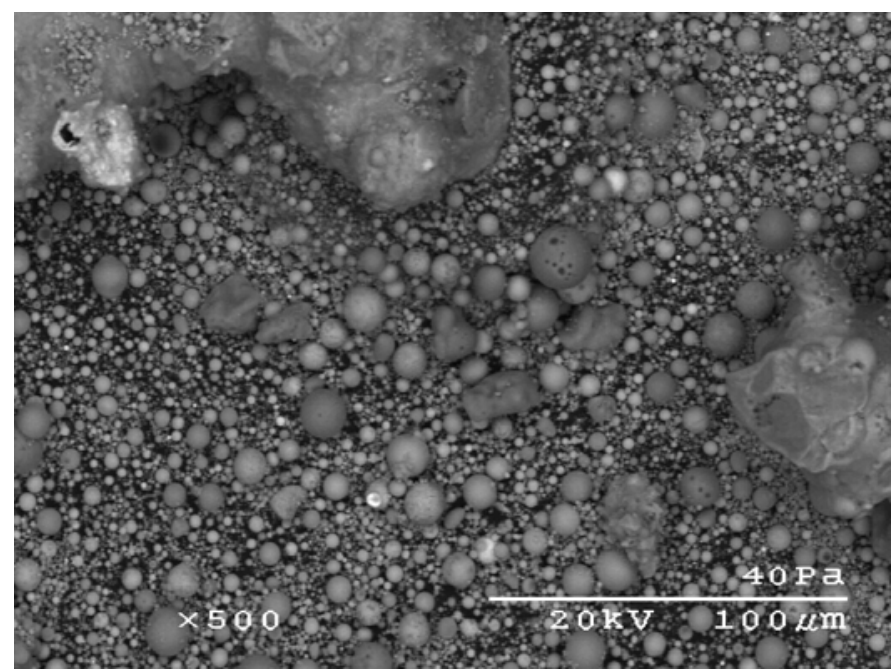

Figure 41 Scanning electron micrograph of coal fly ash

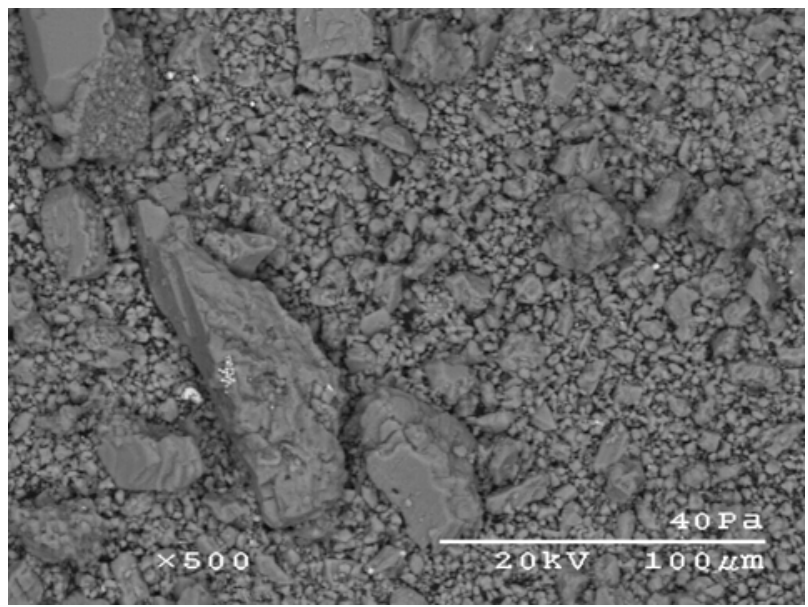

Figure 42 Scanning electron micrograph for limestone

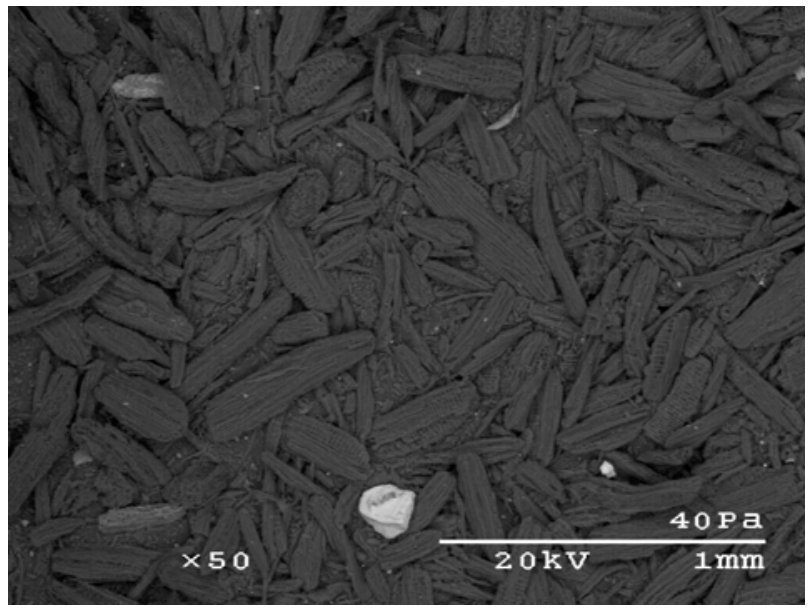

Figure 43 Scanning electron micrograph for oak char 
An irregularly shaped particle is also more likely to be re-entrained in the gas flow once captured by a granule. Irregularly shaped particles produce larger drag forces than more spherical shapes and are more likely to project into the boundary layer surrounding a granule. Viscous effects of the flowing gas, creating relatively thick boundary layers around the granules, dominate the laminar flow typically existing through a granular bed.

Particle density is also important in determining filtration efficiency, as is evident in comparing the filtration efficiency of limestone and cornstarch particles. The two materials have similar size and shape but the limestone particles are almost twice as dense as the cornstarch. The collection efficiency of the limestone was $94.67 \%$ while compared to only $91.15 \%$ for the less dense cornstarch. The higher momentum of denser particles increases the chance of inertial impaction with the granular media ${ }^{22}$.

The low density $\left(560 \mathrm{~kg} / \mathrm{m}^{3}\right)$ and irregular shape of the oak char conspire to produce very low filtration efficiencies for this material compared to the other particulate matter evaluated. Char, consisting mostly of carbon, is expected to have strong Van der Waals forces by virtue of carbon's large Hamaker constant compared to other materials ${ }^{22}$. However, it would appear that this positive effect is overridden by low particle density and irregular particle shape to limit the collection efficiency of oak char to only $66.20 \%$ under the conditions tested.

This study found that a simple particle removal model was able to predict the performance of a moving bed granular filter under development at Iowa State University. The bimodally distributed coal fly ash tested in this study required separate layer efficiencies to be employed in the model for the two sizes of particles. Very fine particles and very coarse particles were the most efficiently removed by the filter while particles in the size range of 33-128 $\mu \mathrm{m}$ appeared to limit overall filter efficiency to about $99 \%$ even for gas residence times in the filter on the order of $1 \mathrm{~s}$. However, these middle-sized particles should be able to be removed by a high efficiency cyclone upstream of the moving bed filter, which would remove fine particles with efficiencies well in excess of $99 \%$. These experiments also showed that in excess of $86 \%$ of the fly ash was removed in the interfacial region where dusty gas first contacts the downward flowing granules of the moving bed.

In tests with other particulate matter (limestone, cornstarch, and oak char) it was demonstrated that filtration efficiency of the moving bed granular filter decreased with decreasing particle density and increasing irregularity of particle shape. Although oak char, consisting mostly of carbon, is expected to have very high van der Waals forces acting, the low density and highly irregular shape of this material resulted in collection efficiencies as low as $66 \%$.

\subsection{Steam Reforming}

The results described in this subsection were published in Energy Conversion and Management. $^{29}$

\section{$\underline{\text { Baseline tar measurements }}$}

When operating in the equivalence ratio range of 0.25 to 0.35 and at gasification temperature of 700 to $760{ }^{\circ} \mathrm{C}$ the average composition of the producer gas was (dry, volumetric basis): $51.2 \%$ 
nitrogen; $15.7 \%$ carbon monoxide; $14.2 \%$ carbon dioxide; $6.5 \%$ hydrogen; $4.8 \%$ methane; and $4 \%$ higher hydrocarbons.

As detailed in Section 2.8, two methods of tar measurement were employed, both based on the methodology developed by the European Union. The evaporation method performed at $105^{\circ} \mathrm{C}$, which gives results that are comparable to other methodologies used to measure "heavy tar," yielded $10.4 \mathrm{~g} / \mathrm{nm}^{3}$ of tar.

The distillation method yields three tar fractions: light tars, heavy tars (distillation residue), and water soluble tars. Applied to the tar samples obtained from the gasifier, the light tar fraction was $13.6 \mathrm{~g} / \mathrm{nm}^{3}$, the heavy tars were $27.8 \mathrm{~g} / \mathrm{nm}^{3}$, and the water soluble tars were $5.7 \mathrm{~g} / \mathrm{nm}^{3}$. Thus, the total carbon concentration arising from hydrocarbons recovered from the impinger train was $47.1 \mathrm{~g} / \mathrm{nm}^{3}$. This number was taken to be a more accurate reflection of the recoverable hydrocarbons in the producer gas. Furthermore, the carbon concentration arising from $\mathrm{CH}_{4}$ and $\mathrm{C}_{2} \mathrm{H}_{4}$ in the producer gas (that is, hydrocarbons not recovered in the impinger train) was 58.9 $\mathrm{g} / \mathrm{nm} 3$. Taken together the appropriate steam/TOC ratio to steam reform hydrocarbons in the producer gas is estimated to be 2.8

\section{$\underline{\text { Tar destruction }}$}

For all catalysts and operating conditions tested, no visible tar was observed in the lines after the catalytic reactor or in the impingers. The dichloromethane mixtures recovered after these tests were clear with no hint of color. Analysis by evaporation at $105^{\circ} \mathrm{C}$ found no detectable tar at the exit of the catalytic reactor for any of the tests, indicating heavy tar reduction in excess of $99 \%$. Analysis by distillation at $75^{\circ} \mathrm{C}$ was performed for only one test: the ICI 46-1 catalyst operated at $800^{\circ} \mathrm{C}$ with a space velocity (SV) of $3000 \mathrm{~h}^{-1}$ and a steam/TOC ratio 2.8. Carbon from the light hydrocarbon fraction was present in the amount of $5.8 \mathrm{~g} / \mathrm{nm}^{3}$ while carbon in the form of soluble hydrocarbons was $0.6 \mathrm{~g} / \mathrm{nm}^{3}$. Although $6.4 \mathrm{~g} / \mathrm{nm}^{3}$ of carbon associated with hydrocarbons recovered in the impinger train may appear to be a relatively large concentration, it includes organic compounds that are not considered "tar" in many applications since they would not normally condense out. Nevertheless, it represents a carbon conversion efficiency of $86 \%$ for hydrocarbons collected from the raw gas by the impinger train operated at $-70^{\circ} \mathrm{C}$.

\section{Effect of Catalytic Reactor Operating Conditions on Gas Composition}

The effects of space velocity, catalytic bed temperature, and steam/C ratio on gas composition $\left(\mathrm{H}_{2}, \mathrm{CO}, \mathrm{CO}_{2}, \mathrm{CH}_{4}\right.$, and $\left.\mathrm{C}_{2} \mathrm{H}_{4}\right)$ for each of the three catalysts are presented in several figures that follow (in all tests, the inlet temperature to the tar destruction system was $650^{\circ} \mathrm{C}$ ). In these figures "GB Inlet" refers to the concentration of a gas species at the guard bed inlet (upstream of the tar destruction system) and "CR Outlet" refers to the concentration of a gas species at the catalytic reactor outlet (downstream of the tar destruction system). In general, $\mathrm{H}_{2}$ and $\mathrm{CO}_{2}$ increase while $\mathrm{CO}$ decreases in the producer gas as it passes through the tar destruction system, as expected for steam reforming reactions acting in tandem with the water-gas shift reaction. Concentrations of $\mathrm{CH}_{4}$ and $\mathrm{C}_{2} \mathrm{H}_{4}$ decrease in the producer gas. The decrease in $\mathrm{CH}_{4}$ was about 0.2-1.0 vol-\% while the decrease in $\mathrm{C}_{2} \mathrm{H}_{4}$ was about $0.5-1.5 \%$. Although these low molecular weight hydrocarbons can be products of steam reforming of tar, they are also susceptible to further steam reforming to $\mathrm{CO}$ and $\mathrm{H}_{2}$. The ICI46-1 catalyst showed no deactivation during 12 


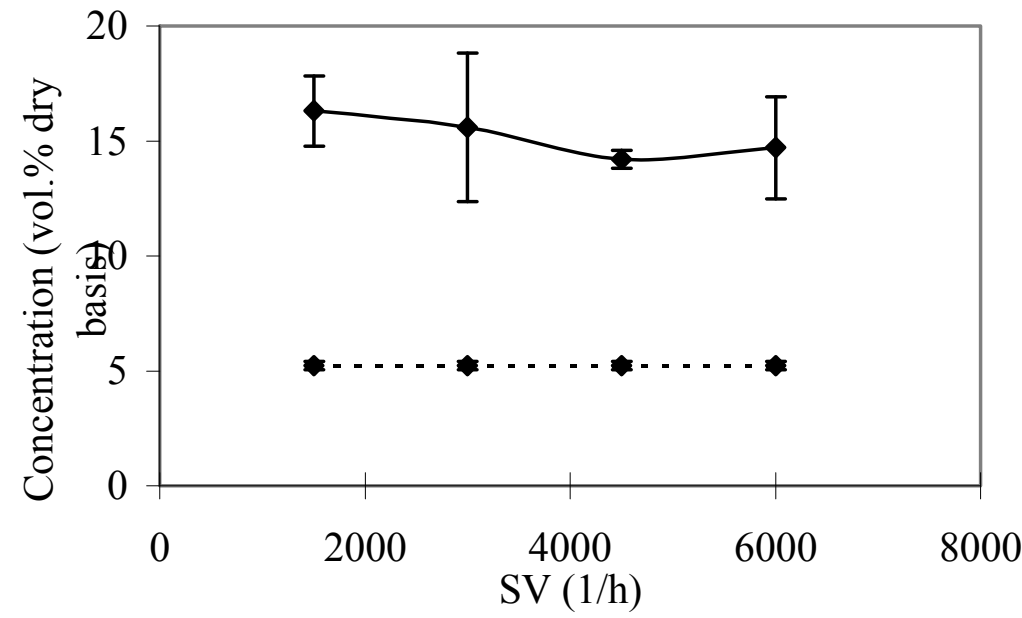

(a)
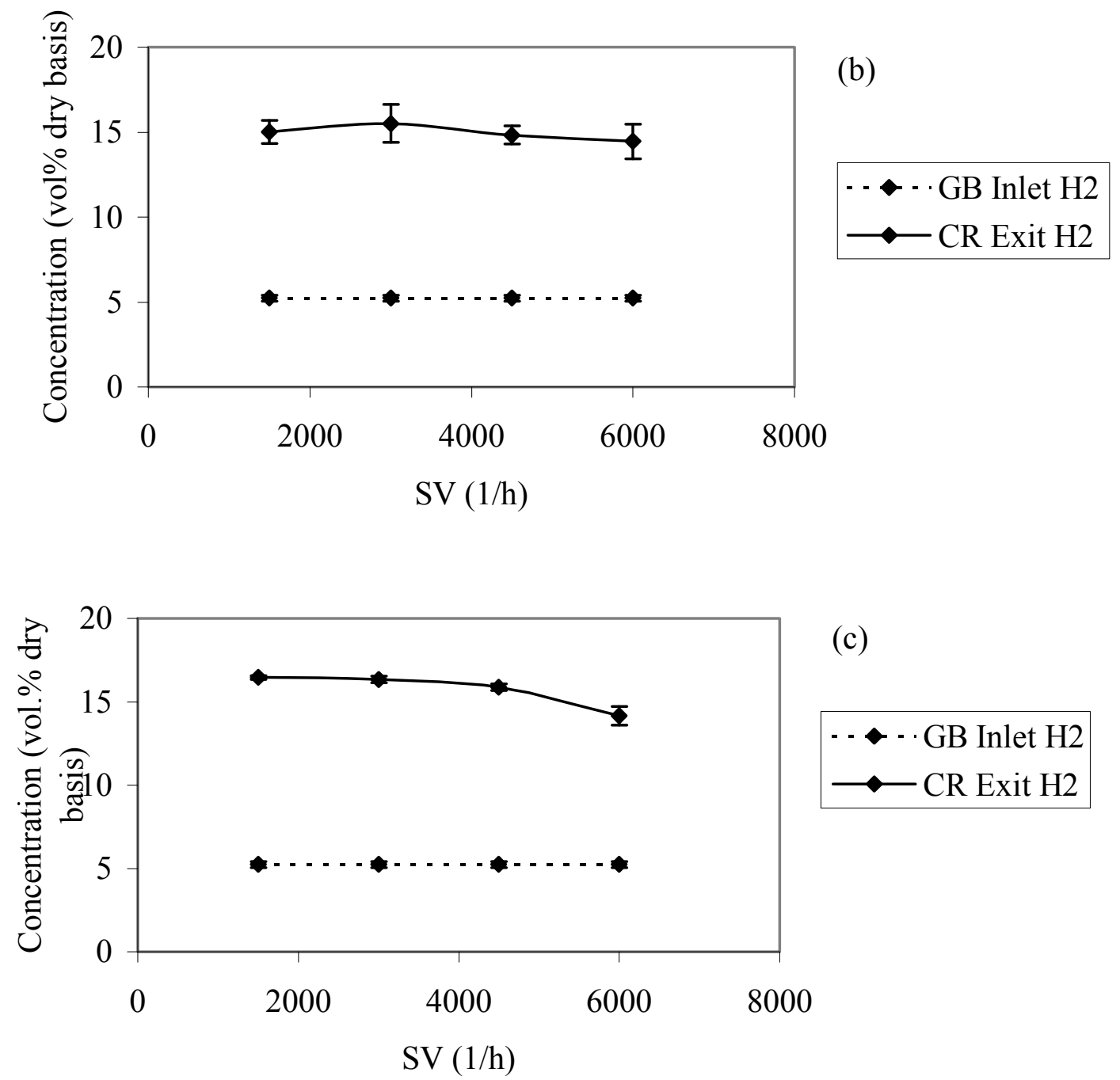

Figure $44 \mathrm{H}_{2}$ concentration in producer gas at the inlet of the guard bed and the exit of catalytic bed as a function of space velocity: $\mathrm{T}_{\mathrm{GB}}=650^{\circ} \mathrm{C} ; \mathrm{T}_{\mathrm{CR}}=800^{\circ} \mathrm{C}$; Steam/TOC = 2.8. (a) ICI46-1, (b) Z409, (c) RZ409. 
hours of testing while the Z409 and RZ409 catalysts showed no deactivation during 18 hours of testing. The effect of space velocity on hydrogen concentration in the producer gas is illustrated in Figure 44 for catalysts ICI46-1, Z409 and RZ409 $\left(\mathrm{T}_{\mathrm{CR}}=800^{\circ} \mathrm{C}\right.$; Steam $/ \mathrm{TOC}=2.8$ ). There was little evidence that decreasing space velocity significantly increases hydrogen production (observed variations are within the uncertainty of hydrogen measurements). The effects of space velocity on $\mathrm{CO}$ and $\mathrm{CO}_{2}$ concentrations in the producer gas are illustrated in Figure 45 for catalysts ICI46-1, Z409 and RZ409. For space velocities less than $4500 \mathrm{~h}^{-1}$ there is no effect on $\mathrm{CO}$ concentration. The concentration of $\mathrm{CO}_{2}$ is not substantially influenced by space velocity in the range of $1500-6000 \mathrm{~h}^{-1}$. The effects of space velocity on $\mathrm{CH}_{4}$ and $\mathrm{C}_{2} \mathrm{H}_{4}$ concentrations in the producer gas are illustrated in Figure 46 for catalysts ICI46-1, Z409 and RZ409. No definitive trends are evident for $\mathrm{CH}_{4}$ while $\mathrm{C}_{2} \mathrm{H}_{4}$ clearly decreases as space velocity decreases. These observations indicate that tar destruction is not mass-transfer limited in the present experimental system.

The effect of catalytic bed temperature on hydrogen concentration in the producer gas is illustrated in Figure 47 for catalysts ICI46-1, Z409 and RZ409 (SV = 3000h ${ }^{-1}$; $\mathrm{Steam} / \mathrm{TOC}=2.8$ ). As expected, hydrogen production increases with increasing reaction temperature although the increase is less than $25 \%$ in going from $740^{\circ} \mathrm{C}$ to $820^{\circ} \mathrm{C}$. The effect of catalytic bed temperature on $\mathrm{CO}$ and $\mathrm{CO}_{2}$ concentrations in the producer gas are illustrated in Figure 48 for catalysts ICI46-1, Z409 and RZ409. Carbon monoxide increases while $\mathrm{CO}_{2}$ decreases with increasing temperature. The strongest effect is observed for catalyst $\mathrm{Z} 409$ (CO increases $40 \%$ in going from $740^{\circ} \mathrm{C}$ to $820^{\circ} \mathrm{C}$ ) and weakest for ICI46-1. The effects of catalytic bed temperature on $\mathrm{CH}_{4}$ and $\mathrm{C}_{2} \mathrm{H}_{4}$ concentrations in the producer gas are illustrated in Figure 49 for catalysts ICI46-1, Z409 and RZ409. No definitive trends are evident for $\mathrm{CH}_{4}$ while $\mathrm{C}_{2} \mathrm{H}_{4}$ clearly decreases especially for the Z409 and RZ409 catalyst (reduction greater than $85 \%$ in going from $740^{\circ} \mathrm{C}$ to $820^{\circ} \mathrm{C}$ ). These observations indicate that the rate of tar destruction is controlled by chemical kinetics.

The effect of steam/TOC ratio on hydrogen concentration in the producer gas is illustrated in Figure 50 for catalysts Z409 and RZ409 $\left(\mathrm{T}_{\mathrm{CR}}=800^{\circ} \mathrm{C} ; \mathrm{SV}=3000 \mathrm{~h}^{-1}\right)$. As expected, hydrogen production increases with increasing steam/TOC ratio although the increase is less $30 \%$ in going from steam/TOC ratio of 2.8 to 6.5 . The effects of steam/TOC ratio on $\mathrm{CO}$ and $\mathrm{CO}_{2}$ concentrations in the producer gas are illustrated in Figure 51 for catalysts Z409 and RZ409. Carbon monoxide decreases by $50 \%$ while $\mathrm{CO}_{2}$ increases by $50 \%$ in going from steam/TOC ratio of 2.8 to 6.5 for both catalysts, indicating a strong water-gas shift reaction. The effects of steam/TOC ratio on $\mathrm{CH}_{4}$ and $\mathrm{C}_{2} \mathrm{H}_{4}$ concentrations in the producer gas are illustrated in Figure 52 for catalysts Z409 and RZ409. No definitive trends are evident for either $\mathrm{CH}_{4}$ or $\mathrm{C}_{2} \mathrm{H}_{4}$.

One reason for evaluating both Z409 and RZ409 catalysts was to determine whether reducing the catalyst prior to use on gasification streams was important to catalytic activity (RZ409 catalyst is pre-reduced Z409). During tests we observed hydrogen concentrations exiting the tar destruction system were 2.0 - 3.0 vol-\% higher for RZ409 


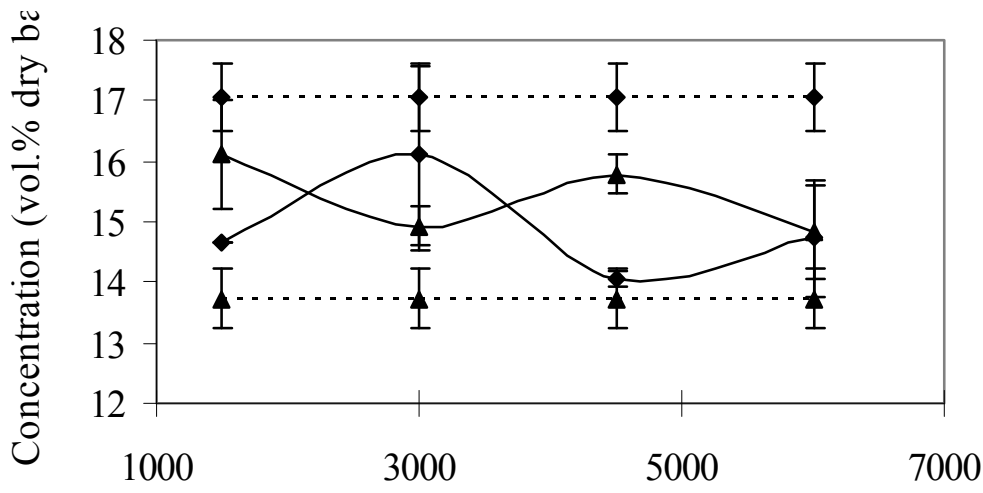

(a)

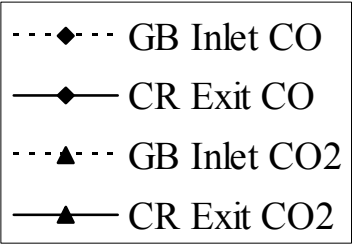

$\mathrm{SV}(1 / \mathrm{h})$
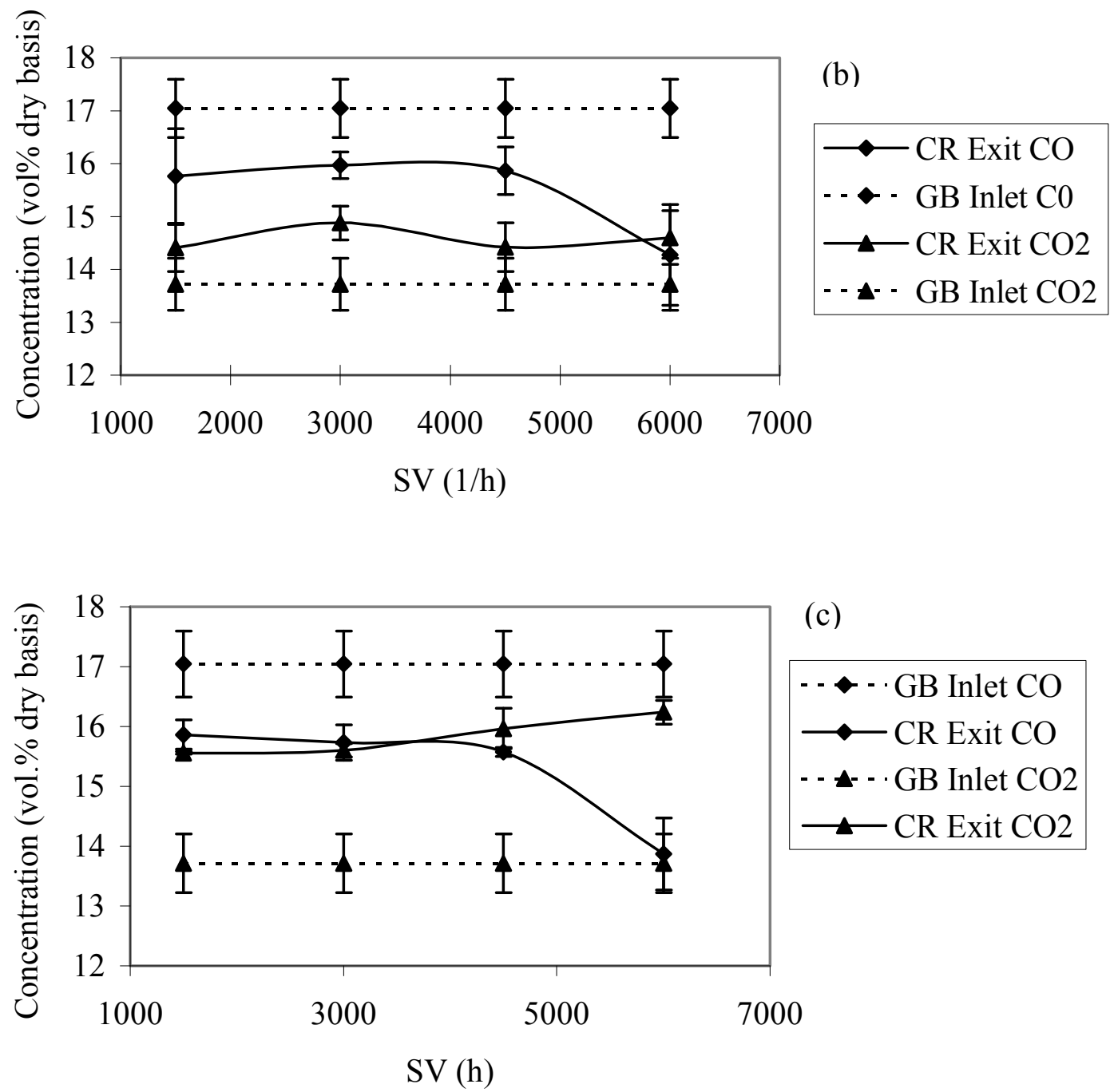

Figure $45 \mathrm{CO}$ and $\mathrm{CO}_{2}$ concentrations in the producer gas at the inlet of the guard bed and the exit of catalytic bed as functions of space velocity: $\mathrm{T}_{\mathrm{GB}}=650^{\circ} \mathrm{C} ; \mathrm{T}_{\mathrm{CR}}=800^{\circ} \mathrm{C}$; Steam/TOC $=2.8$. (a) ICI46-1, (b) Z409, (c) RZ409. 

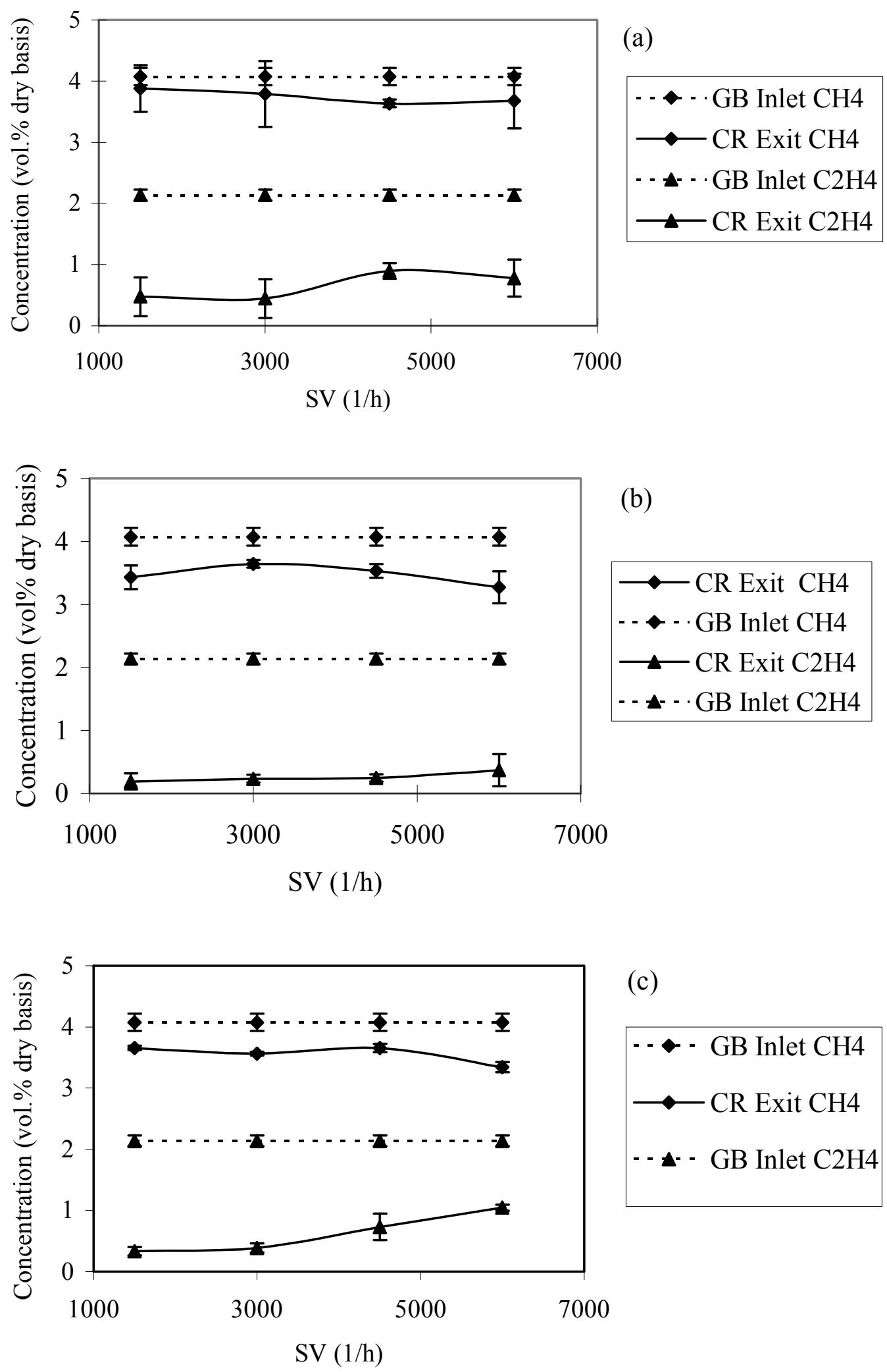

(c)

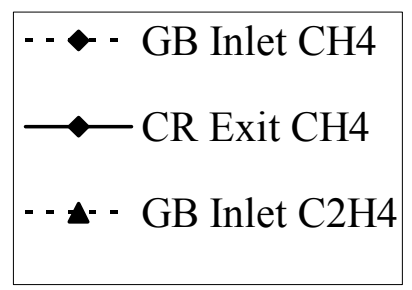

Figure $46 \mathrm{CH}_{4}$ and $\mathrm{C}_{2} \mathrm{H}_{4}$ concentrations in the producer gas at the inlet of the guard bed and the exit of catalytic as functions of space velocity: $\mathrm{T}_{\mathrm{GB}}=650^{\circ} \mathrm{C} ; \mathrm{T}_{\mathrm{CR}}=$ $800^{\circ} \mathrm{C}$ : Steam/TOC $=2.8$. (a) ICI46-1. (b) Z409. (c) RZ409. 


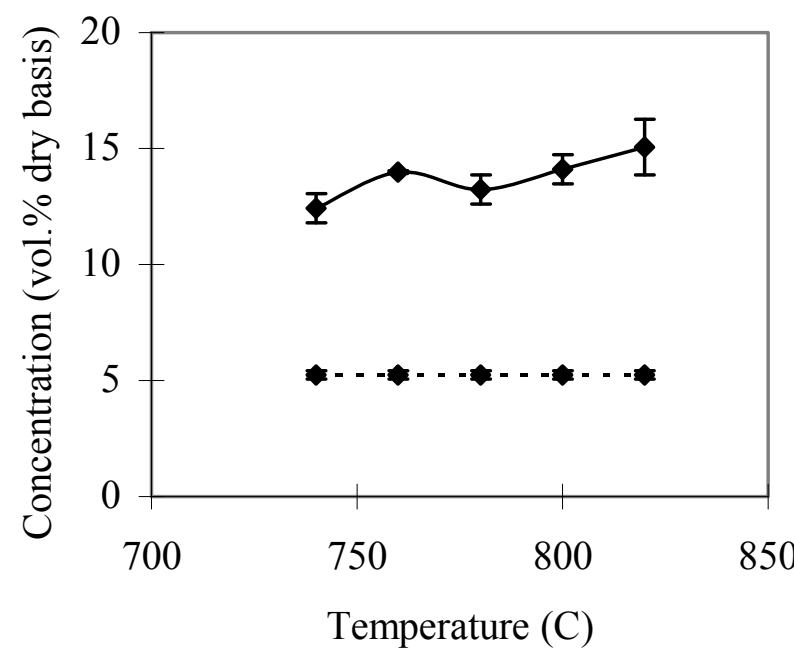

(a)
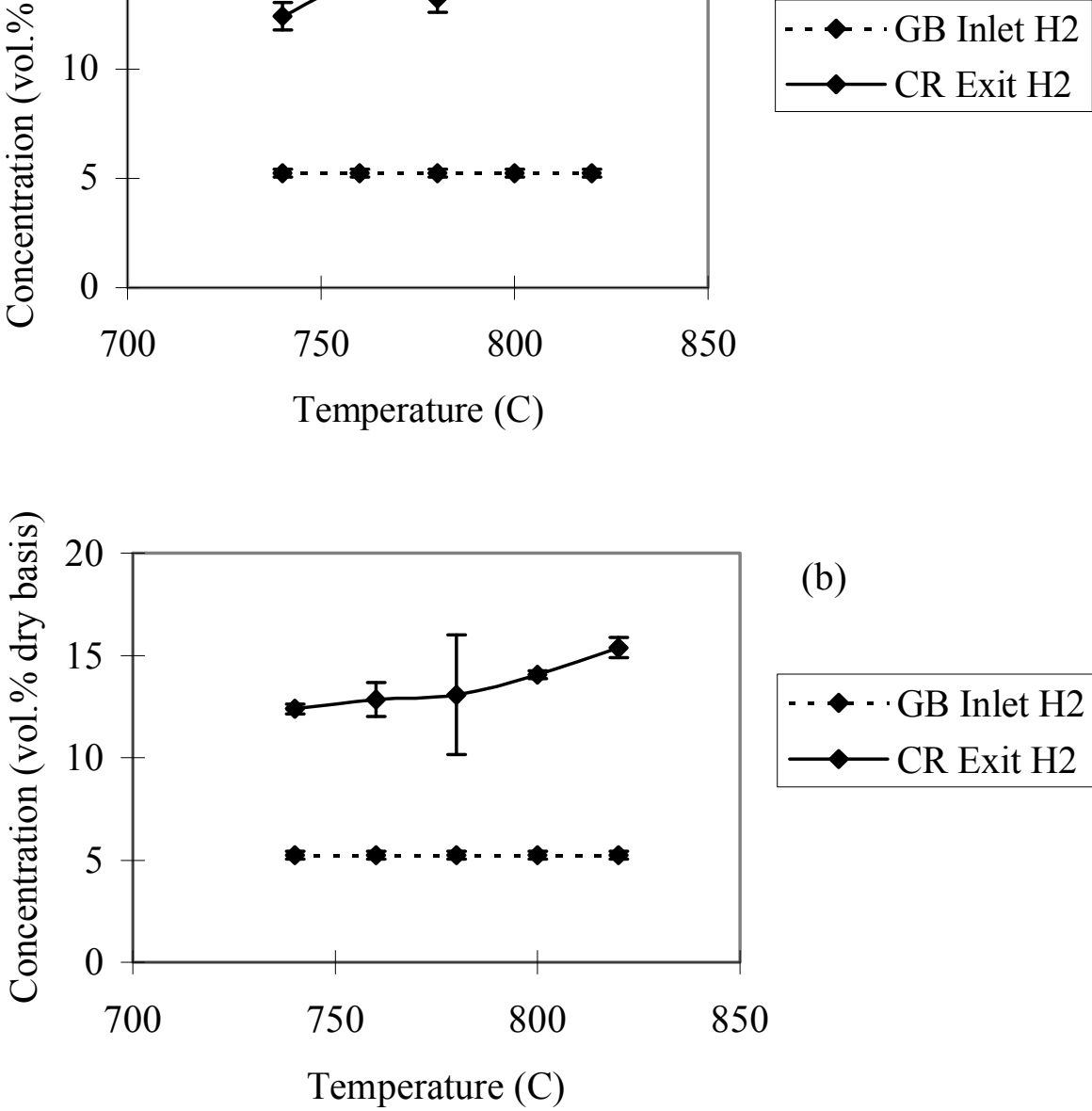

(b)
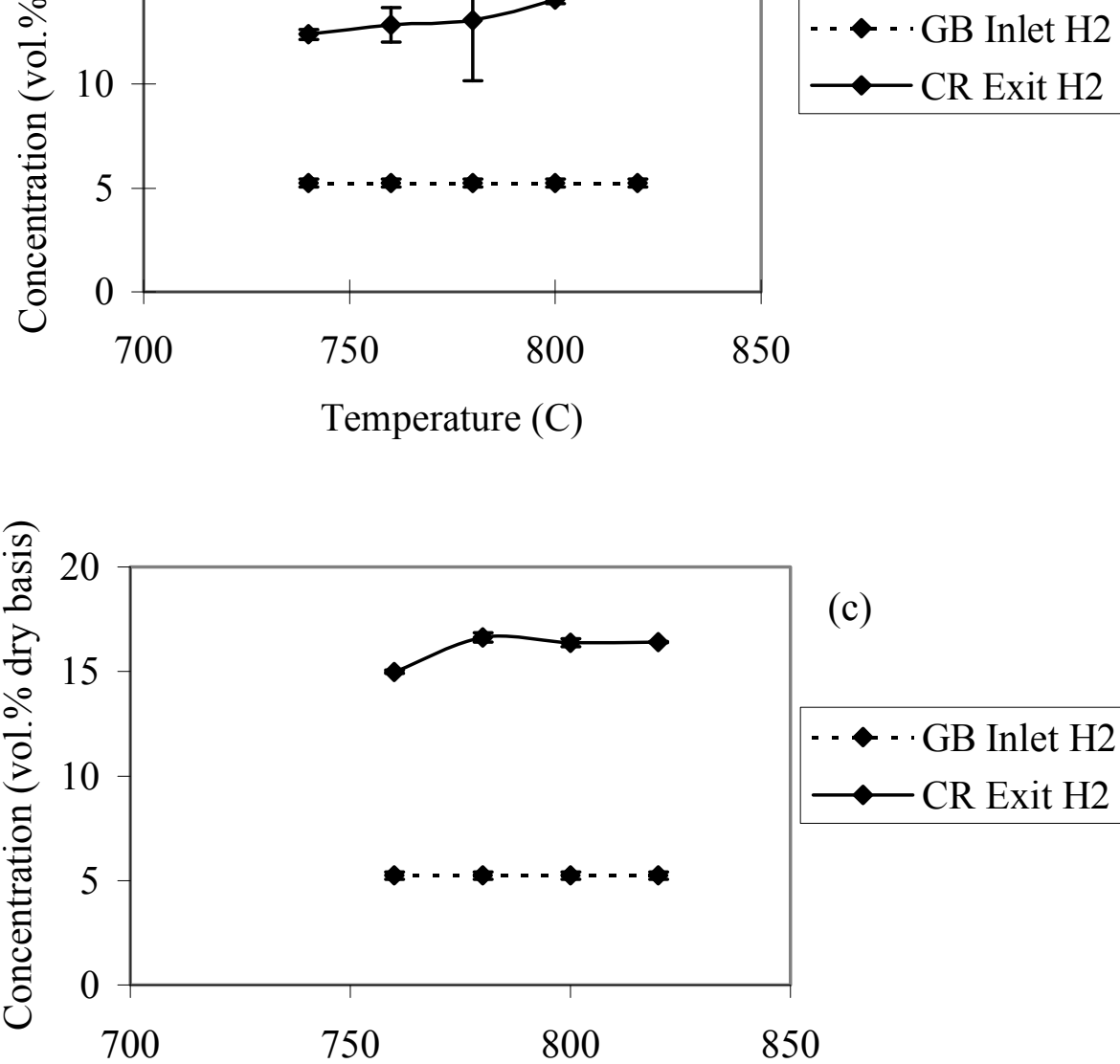

Temperature (C)

Figure $47 \mathrm{H}_{2}$ concentration in the producer gas at the inlet of the guard bed and the exit of catalytic bed as a function of catalytic bed temperature: $\mathrm{T}_{\mathrm{GB}}=650^{\circ} \mathrm{C}$; $\mathrm{SV}=3000 \mathrm{~h}^{-1}$; Steam/TOC $=2.8$. (a) ICI46-1, (b) Z409, (c) RZ409. 


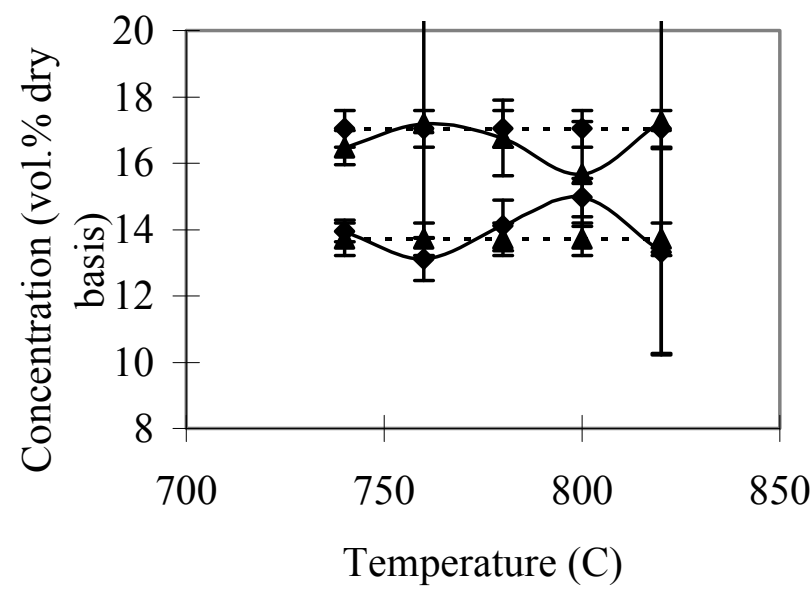

(a)
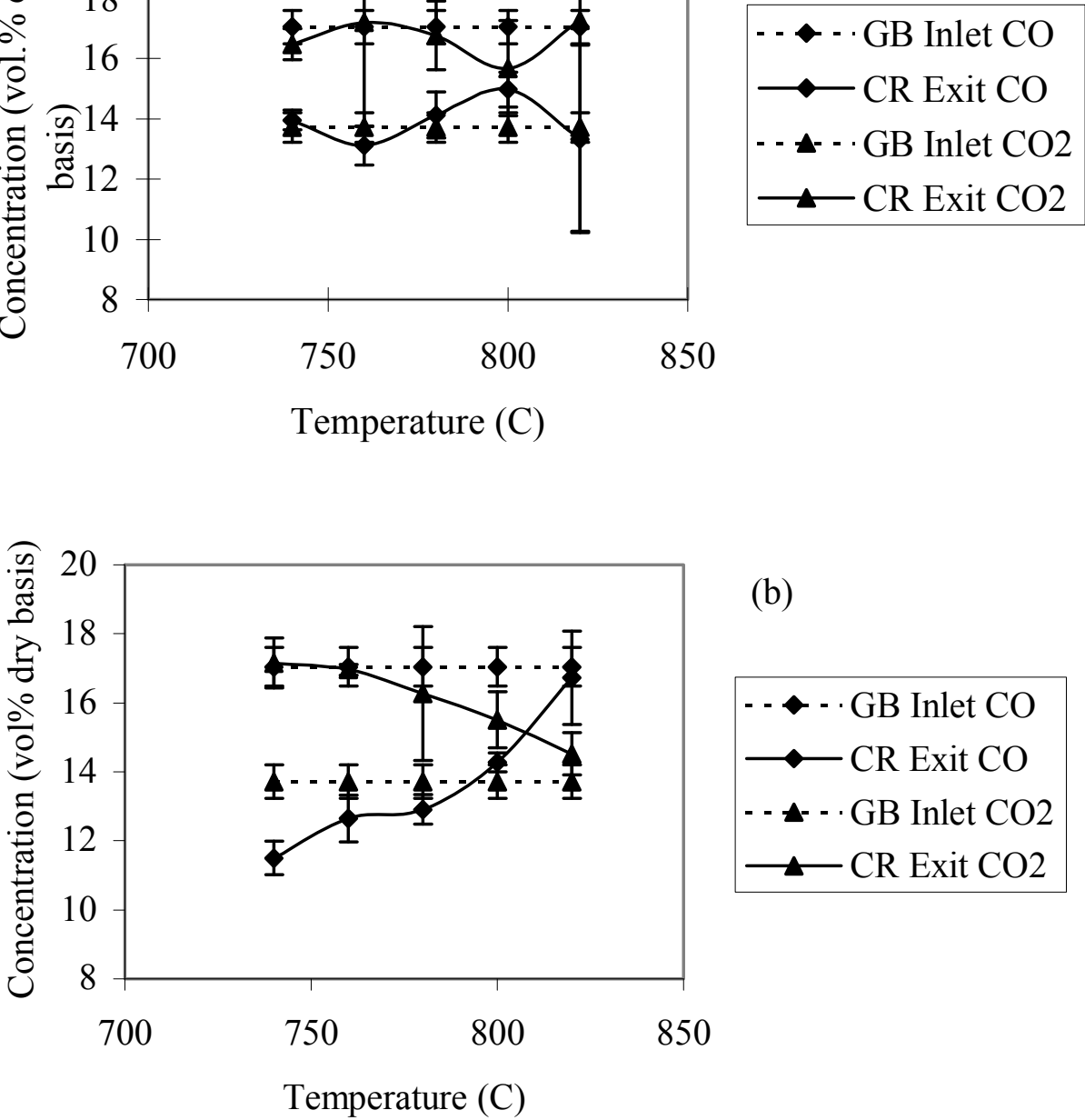

(b)
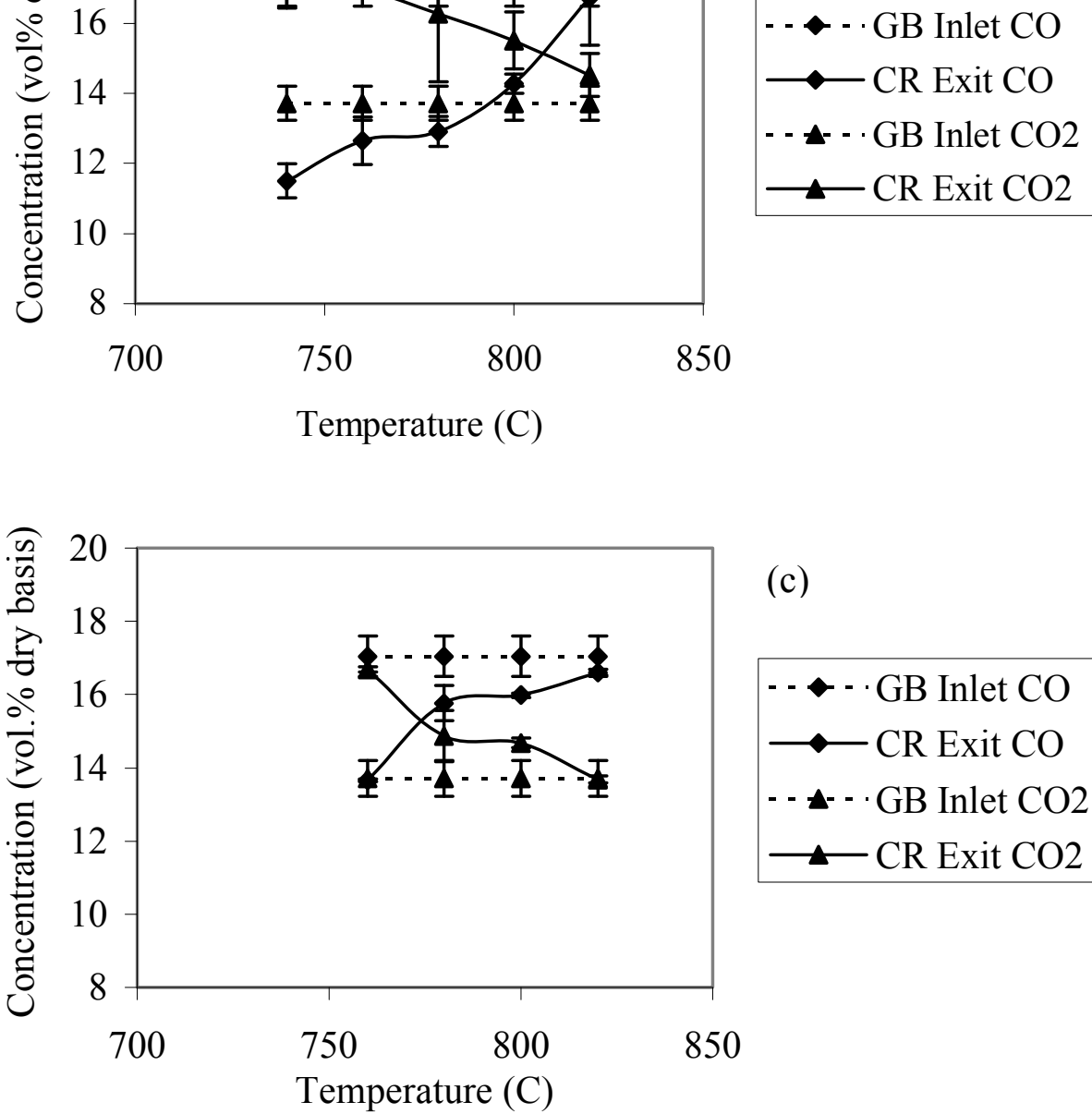

(c)

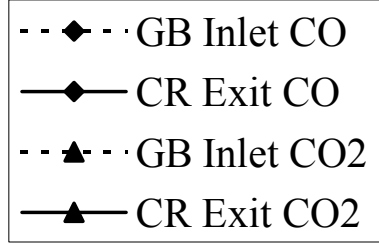

Figure $48 \mathrm{CO}$ and $\mathrm{CO}_{2}$ concentrations in the producer gas at the inlet of the guard bed and the exit of catalytic bed as functions of catalytic bed temperature: $\mathrm{T}_{\mathrm{GB}}$ $=650^{\circ} \mathrm{C} ; \mathrm{SV}=3000 \mathrm{~h}^{-1} ;$ Steam $/ \mathrm{TOC}=2.8$. (a) ICI46-1, (b) Z409, (c) 


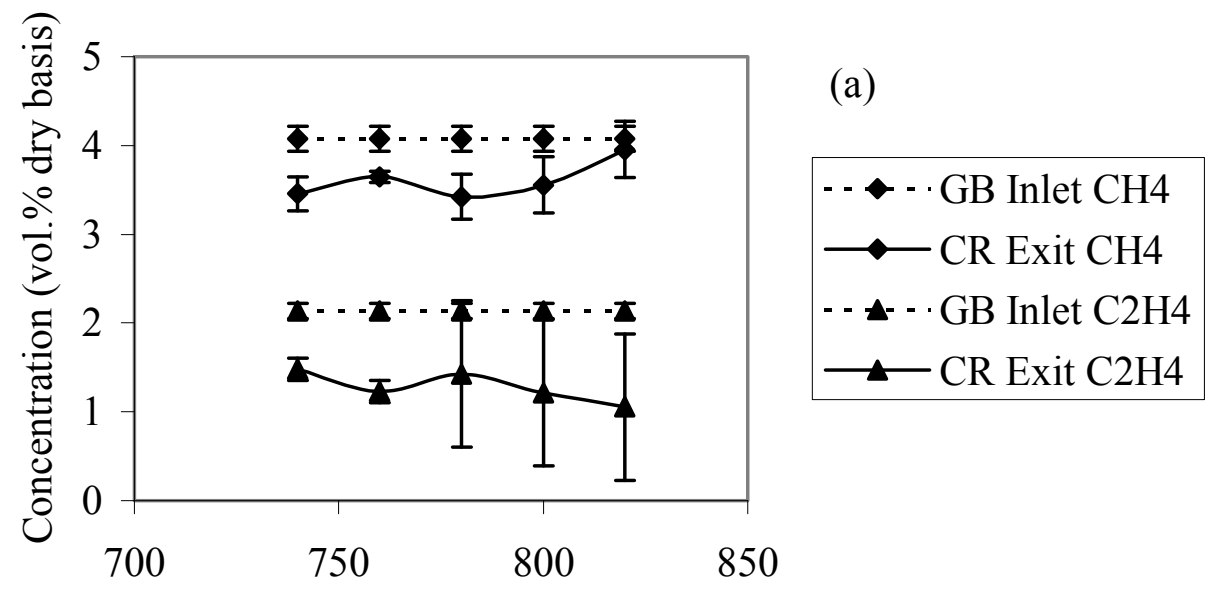

Temperature (C)

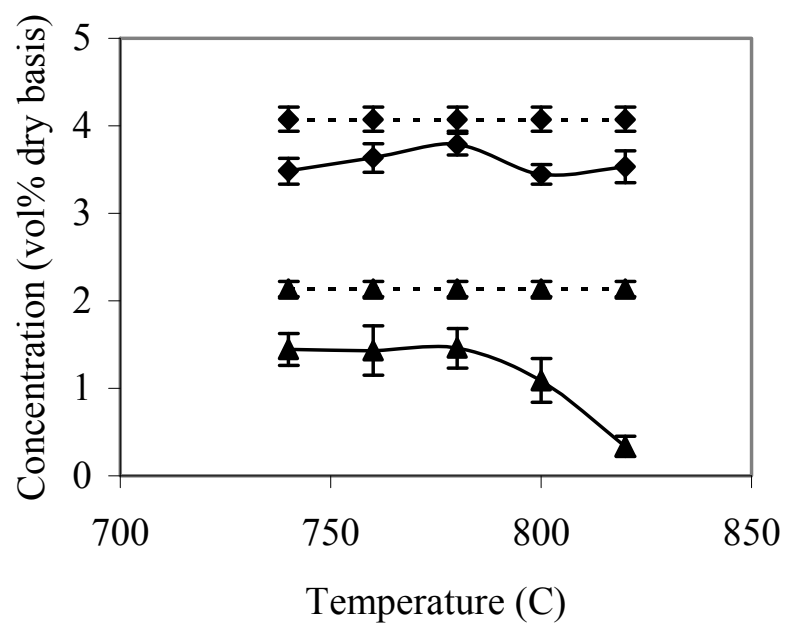

(b)
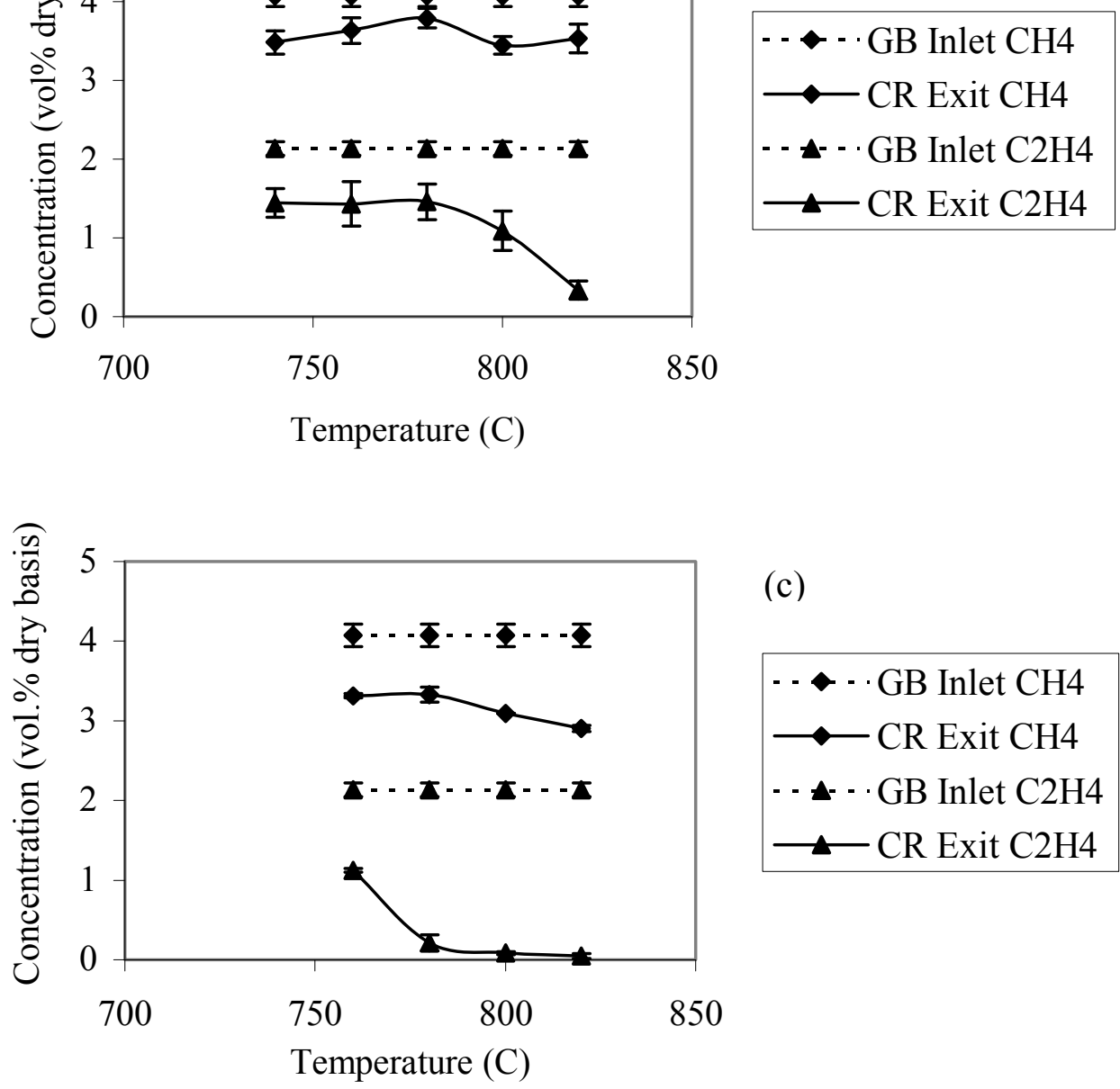

(c)

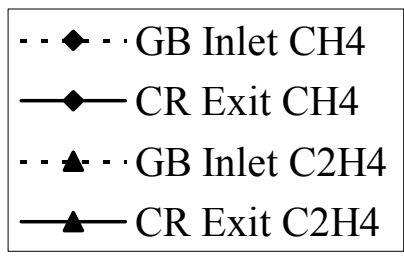

Figure $49 \mathrm{CH}_{4}$ and $\mathrm{C}_{2} \mathrm{H}_{4}$ concentrations in the producer gas at the inlet of the guard bed and the exit of catalytic bed as functions of catalytic bed temperature: $\mathrm{T}_{\mathrm{GB}}=650^{\circ} \mathrm{C} ; \mathrm{SV}=3000 \mathrm{~h}^{-1} ; \mathrm{Steam} / \mathrm{TOC}=2.8$. (a) ICI46-1, (b) Z409, (c) 


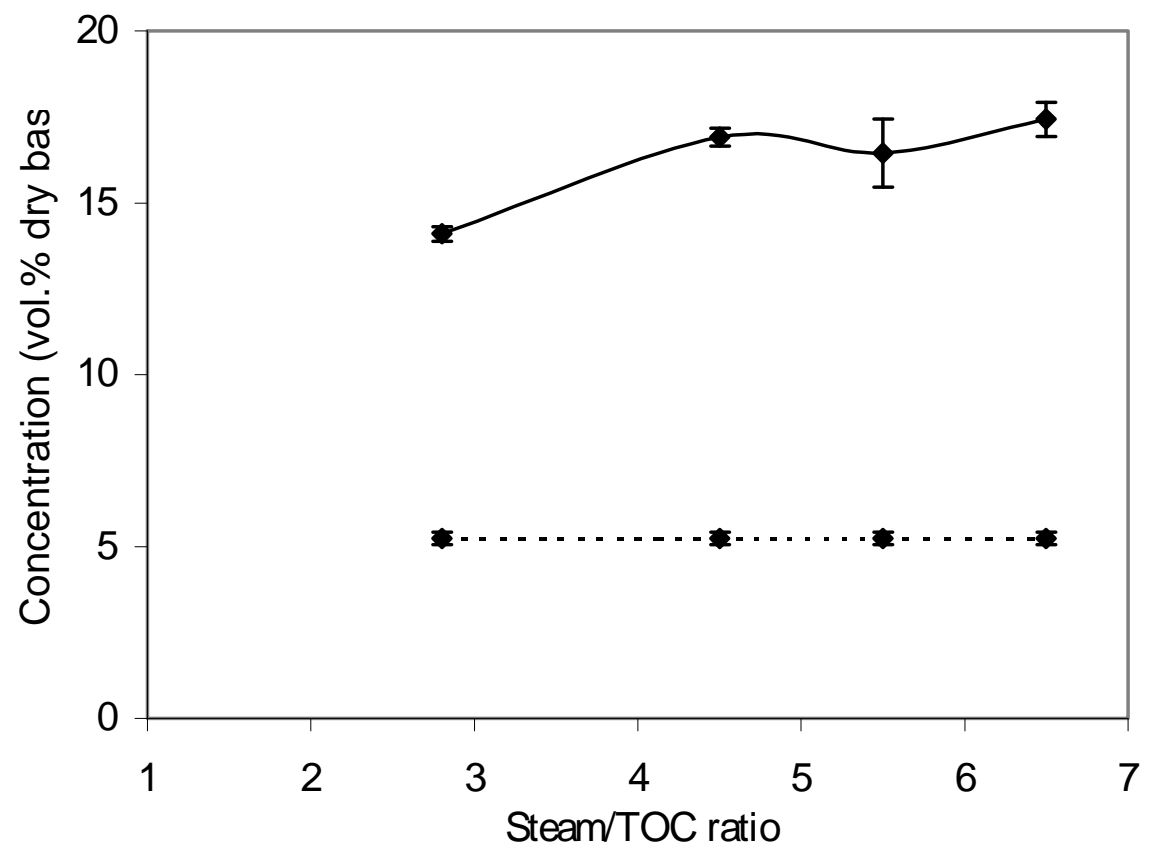

(a)
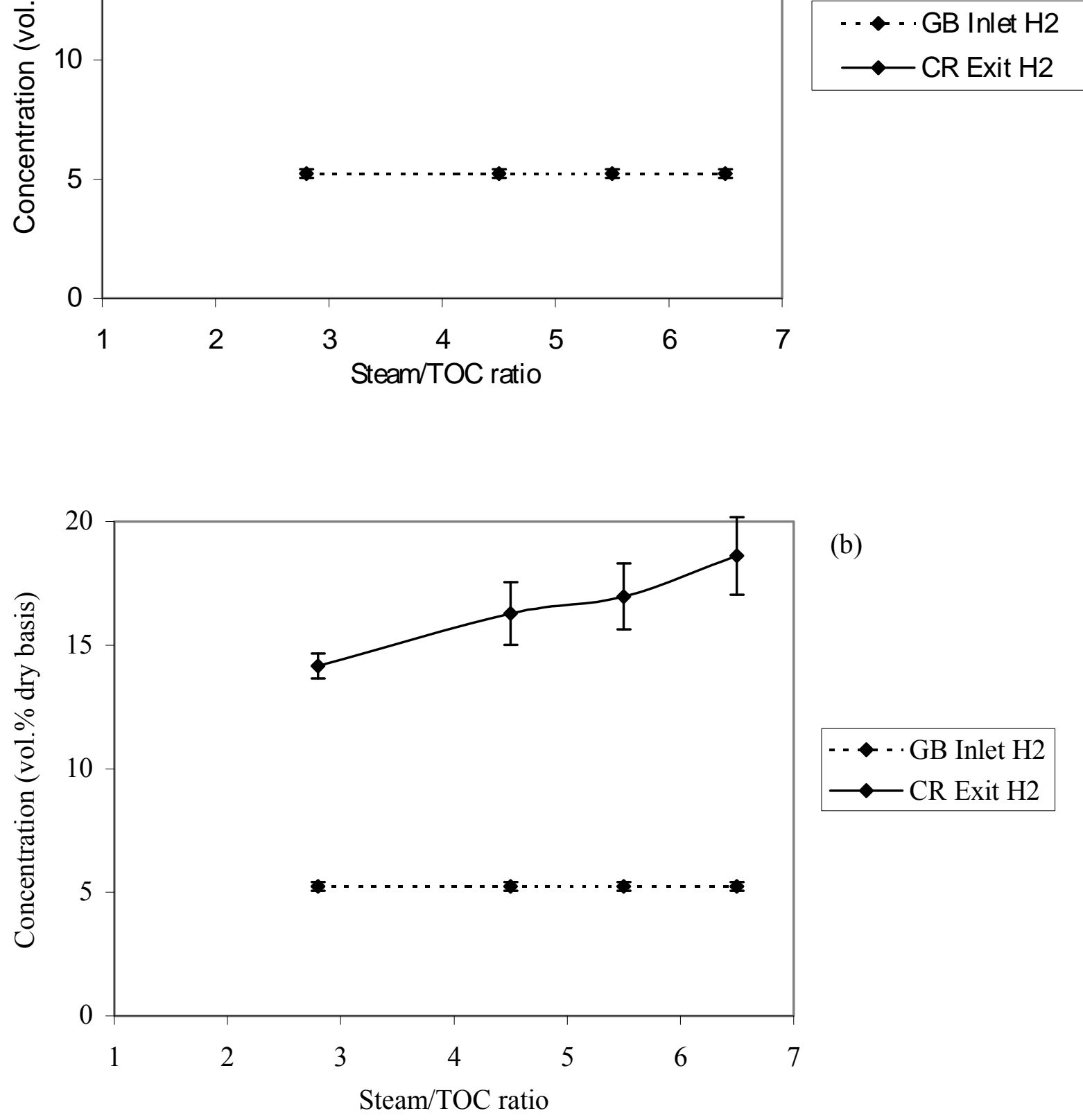

(b)

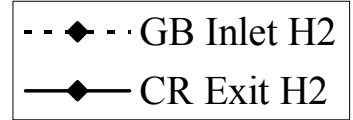

Figure $50 \mathrm{H}_{2}$ concentration in producer gas at the inlet of the guard bed and the exit of catalytic bed as a function of steam $/ \mathrm{TOC}$ ratio: $\mathrm{T}_{\mathrm{GB}}=650^{\circ} \mathrm{C} ; \mathrm{T}_{\mathrm{CR}}=800^{\circ} \mathrm{C}$; $\mathrm{SV}=3000 \mathrm{~h}^{-1}$. (a) Z409, (b) RZ409. 

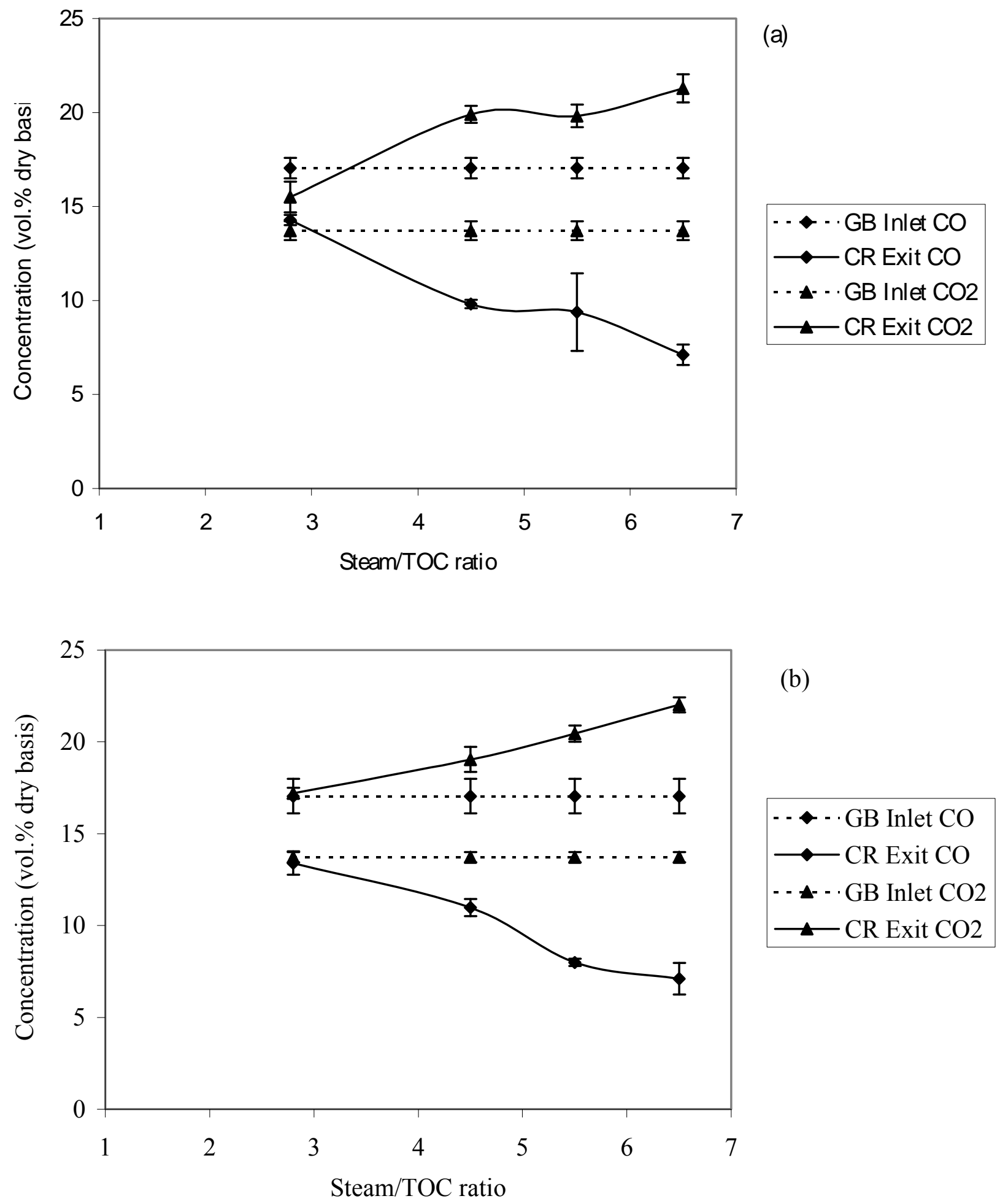

Figure $51 \mathrm{CO}$ and $\mathrm{CO}_{2}$ concentrations in producer gas at the inlet of the guard bed and the exit of catalytic bed as functions of steam $/ \mathrm{TOC}$ ratio: $\mathrm{T}_{\mathrm{GB}}=650^{\circ} \mathrm{C}$; $\mathrm{T}_{\mathrm{CR}}=800^{\circ} \mathrm{C} ; \mathrm{SV}=3000 \mathrm{~h}-1$. (a) Z409, (b) RZ409. 

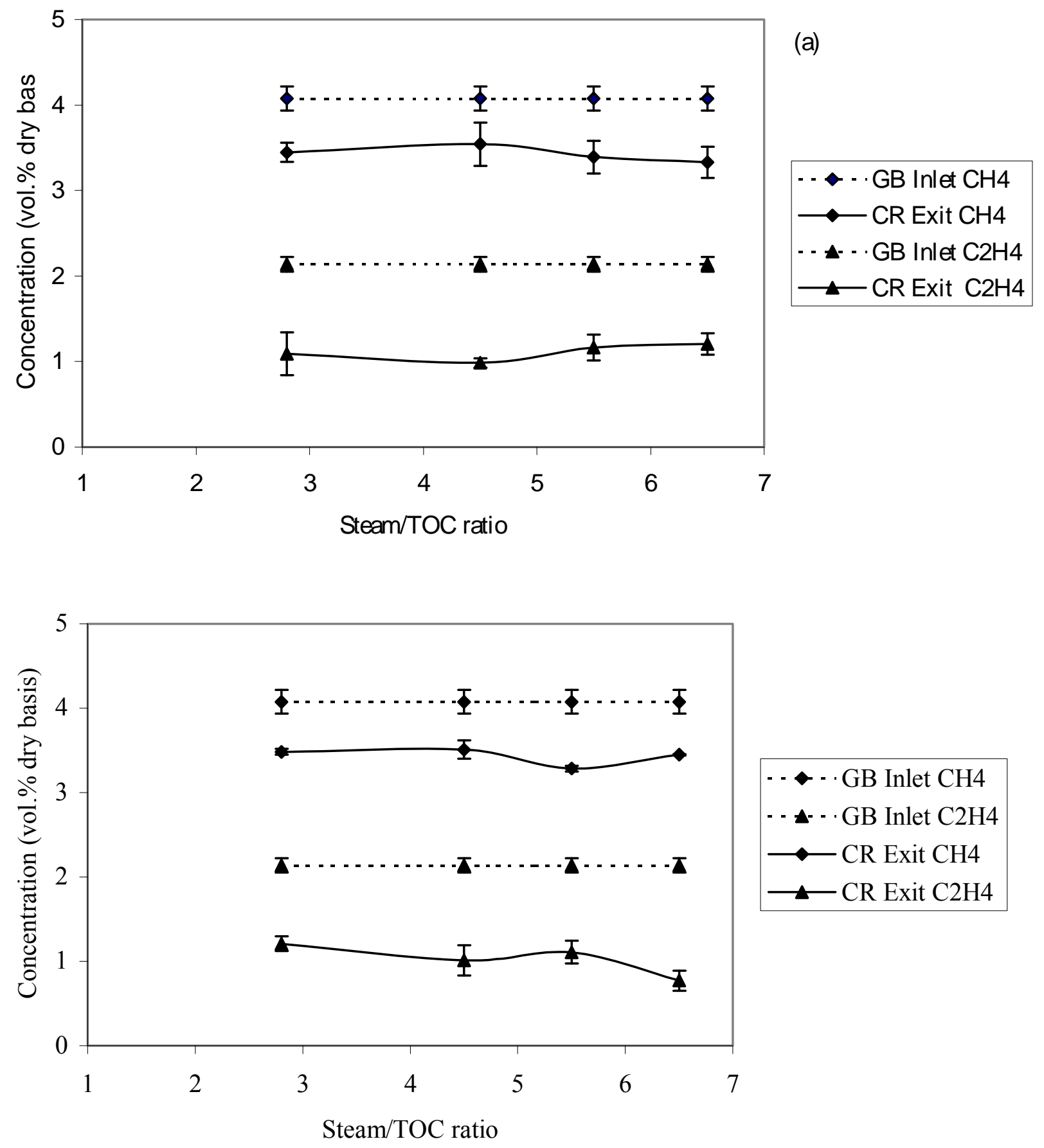

Figure $52 \mathrm{CH}_{4}$ and $\mathrm{C}_{2} \mathrm{H}_{4}$ concentrations in producer gas at the inlet of the guard bed and the exit of catalytic bed as functions of steam $/ \mathrm{TOC}$ ratio: $\mathrm{T}_{\mathrm{GB}}=650^{\circ} \mathrm{C} ; \mathrm{T}_{\mathrm{CR}}=800^{\circ} \mathrm{C}$; $\mathrm{SV}=3000 \mathrm{~h}^{-1}$. (a) Z409, (b) RZ409. 
than for Z409 during the first 2 to 3 hours. However, for longer times the difference between them disappeared. Thus, it appears that the producer gas is able to quickly reduce the metallic catalysts, making unnecessary a separate reducing step before using the catalyst.

\section{Mercury Porosimetry Analysis}

The catalysts were analyzed by mercury porosimetry to compare surface areas, pore sizes and pore size distributions before and after use in the tar destruction system (fresh and used catalyst, respectively). The results are shown in Table 15. In all cases, the pore structure of used catalysts changed.

The ICI 46-1 catalyst showed insignificant change in surface area while the Z409 and RZ409 catalysts showed surface area reductions of 30-35\%. Furthermore, all three catalysts showed

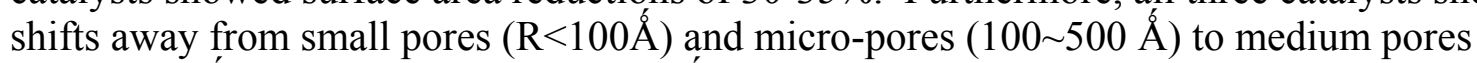
(500 2000 $\AA$ ) and large pores $(\mathrm{R}>2000 \AA$ ). Although this could result from coke blocking the smaller pores, the fact that pore volume increased suggests the conversion of small pores and micro-pores into larger pores during high temperature operation. If this transformation were to continue, catalytic activity would eventually degrade.

Carbon and sulfur analysis of catalysts and dolomite

Carbon and sulfur analyses were performed on each of the three metallic catalysts and the dolomite catalyst both before and after the catalysis tests. Since all of the catalysts are inorganic, the appearance of carbon is an indication of coking. Likewise, the accumulation of sulfur on the metal catalyst indicates the breakthrough of hydrogen sulfide from the guard bed. The results are listed in Table 16.

Although the metallic catalysts were selected for their high resistance to carbon deposition, both the metallic and mineral catalysts accumulated carbon. However, the accumulation on the dolomite bed was 6 to 20 times greater than on the metallic catalysts, suggesting that the guard bed was doing its job of cracking the heaviest tar compounds, which are most likely to produce coking.

Steam/TOC ratios of 4-6 are typically used in Ni-based catalytic steam reforming of naphtha. In our tests the first several hours of testing for all the catalysts were performed without steam injection; that is, only steam arising from biomass gasification was present. This resulted in steam/TOC ratios of only 2.8. In an effort to remove coke accumulated after $18 \mathrm{~h}$ of testing, the steam/TOC ratio of the producer gas was increased to $4-6$ for the last six hours of testing of the Z409 and RZ409 catalysts. Although higher steam levels may enhance destruction of hydrocarbons absorbed on the catalysts, we saw no evidence that coke already deposited was readily removed by the steam/carbon reaction.

We hoped that the calcined dolomite in the guard bed would absorb most of the hydrogen sulfide existing in the producer gas. However, the appearance of sulfur in all the samples of used metallic catalysts and the relatively low concentration of sulfur in the used dolomite indicates significant breakthrough of hydrogen sulfide from the guard bed. In fact, the high concentration of sulfur in the used ICI46-1 catalyst ( $0.4 \mathrm{wt}-\%$ after $12 \mathrm{~h}$ without steam injection) indicates a 
Table 15 Pore volume, specific surface and pore size distribution of catalyst samples

\begin{tabular}{|l|l|l|l|l|l|l|}
\hline & \multirow{2}{*}{$\begin{array}{l}\text { Sample No. } \\
\text { volume }\end{array}$} & \multirow{2}{*}{$\begin{array}{l}\text { Specific } \\
\left(\mathrm{cm}^{3} / \mathrm{g}\right)\end{array}$} & \multicolumn{5}{|c|}{$\begin{array}{l}\text { Surface } \\
\left(\mathrm{m}^{2} / \mathrm{g}\right)\end{array}$} & & $\mathrm{R}<100 \AA$ & $100 \sim 500 \AA$ & $500 \sim 2000 \AA$ & $\mathrm{R}>2000 \AA$ \\
\hline \hline Fresh ICI 46-1 & 0.17 & 16.46 & 13 & 32 & 26 & 29 \\
\hline Used ICI46-1 & 0.21 & 16.22 & 10 & 18 & 37 & 35 \\
\hline Fresh Z409 & 0.14 & 22.92 & 34 & 38 & 22 & 6 \\
\hline Used Z409 & 0.23 & 15.99 & 9 & 28 & 49 & 14 \\
\hline Fresh RZ409 & 0.18 & 23.32 & 28 & 34 & 34 & 4 \\
\hline Used RZ409 & 0.21 & 14.78 & 11 & 24 & 43 & 22 \\
\hline \hline
\end{tabular}

Table 16 Carbon and sulfur analysis of metallic catalysts and dolomite

\begin{tabular}{|l|l|l|l|}
\hline Sample & $\mathrm{S}(\mathrm{wt} \%)$ & $\mathrm{C}(\mathrm{wt} \%)$ & Condition \\
\hline \hline Fresh ICI 46-1 & 0.016 & $\sim 0$ & Fresh \\
\hline Used ICI 46-1 & 0.4 & 0.36 & $12 \mathrm{hr}$ run (no injected steam) \\
\hline \hline Fresh Z409 & 0.013 & $\sim 0$ & Fresh catalyst \\
\hline Used Z409 & 0.021 & 0.80 & $18 \mathrm{hr}+6 \mathrm{hr}$ with injected steam \\
\hline \hline Fresh RZ409 & 0.018 & $\sim 0$ & Fresh catalyst \\
\hline Used RZ409 & 0.019 & 1.04 & $18 \mathrm{hr}+6 \mathrm{hr}$ with injected steam \\
\hline \hline Fresh Dolomite & 0.0084 & $\sim 0$ & Fresh \\
\hline Used Dolomite 1 & 0.014 & 7.26 & $12 \mathrm{hr}$ (no injected steam) \\
\hline Used Dolomite 2 & 0.012 & 6.46 & $18 \mathrm{hr}$ (no injected steam) \\
\hline Used Dolomite 3 & 0.013 & 6.76 & $18 \mathrm{hr}$ (no injected steam) \\
\hline \hline
\end{tabular}


very serious problem. However, relatively little sulfur accumulated on the used Z409 and RZ409 catalysts, which were subjected to steam injection for the last $6 \mathrm{~h}$ of testing. This observation suggests that steam injection can regenerate metallic catalysts that have been poisoned by sulfur. The regenerative process may consist of the following three reactions:

Eq. 18

$$
\mathrm{NiS}+\mathrm{H} 2 \mathrm{O} \rightarrow \mathrm{NiO}+\mathrm{H} 2 \mathrm{~S}
$$

Eq. 19

$$
\mathrm{NiO}+\mathrm{H} 2 \rightarrow \mathrm{Ni}+\mathrm{H} 2 \mathrm{O}
$$

Eq. 20

$$
\mathrm{NiO}+\mathrm{CO} \rightarrow \mathrm{Ni}+\mathrm{CO} 2
$$

After as little as $6-8 \mathrm{~h}$ of testing, a white powder was found in the tar sampling line after the catalytic reactor. This proved to be dolomite that had attrited in the guard bed and blown through the slipstream line. Clearly, the strength of catalytic material for the guard bed needs to be improved.

\subsection{Water Gas Shift Reaction}

Two methods for increasing the hydrogen content of producer gas were investigated: conventional water-gas shift catalysts, consisting of both high temperature and low temperature shift reactors, and a combined catalyst/sorbent system that would simultaneously perform the water gas shift reaction and absorb the carbon dioxide released by this reaction in a single reactor.

The conventional water-gas shift catalysts were tested with producer gas from a biomass gasifier while the combined catalyst/sorbent system was tested with synthetic syngas. Since producer gas contains inorganic contaminants (sulfur and chlorine) and tar, the evaluations of the conventional water-gas shift catalysts had to be performed in combination with the guard bed and steam reforming reactor that are part of the overall gas conditioning system. The results described in this subsection were published in Energy and Fuels ${ }^{30}$ or will appear in Fuel Processing and Technology in early $2005 .^{31}$

\section{Conventional Water-Gas Shift Catalysts}

The first evaluation was to determine the nominal performance of the gas conditioning system. These results are shown in Table 17. The raw producer gas entering the system contained 8.6 vol- $\% \mathrm{H}_{2}, 14.3$ vol- $\% \mathrm{CO}, 18.0$ vol- $\% \mathrm{CO}_{2}, 4.5$ vol- $\% \mathrm{CH}_{4}$, and 1.5 vol- $\% \mathrm{C}_{2} \mathrm{H}_{4}$. The producer gas contained about $19.5 \mathrm{~g} / \mathrm{Nm}^{3}$ heavy tar.

Exiting the steam reformer, the average gas composition was: 19.4 vol- $\% \mathrm{H}_{2}, 9.0$ vol- $\% \mathrm{CO}$, 20.5 vol- $\% \mathrm{CO}_{2}, 3.36$ vol- $\% \mathrm{CH}_{4}$, and 0.28 vol- $\% \mathrm{C}_{2} \mathrm{H}_{4}$. No condensable (heavy) tar was detectable at the exit of the steam reformer. As might be expected, the reaction between steam and tar increased the hydrogen content of the producer gas. Although steam reforming might also be expected to produce $\mathrm{CO}$, this gas actually decreased 5.3 vol- $\%$, indicating that the water- 
Table 17 Producer gas composition at various locations in the gas conditioning system

\begin{tabular}{|c|c|c|c|c|}
\hline & Raw gas & $\begin{array}{c}\text { Outlet steam } \\
\text { reformer }\end{array}$ & $\begin{array}{c}\text { Outlet high } \\
\text { temperature shift }\end{array}$ & $\begin{array}{c}\text { Outlet low } \\
\text { temperature shift }\end{array}$ \\
\hline Gas Composition & & & & $23.7 \pm 0.1$ \\
$\mathrm{H}_{2}$ & $8.6 \pm 0.2$ & $19.4 \pm 0.2$ & $1.4 \pm 0.5$ & $26.7 \pm 1.9$ \\
$\mathrm{CO}$ & $14.3 \pm 0.5$ & $9.0 \pm 0.2$ & $26.8 \pm 0.1$ & $27.4 \pm 0.03$ \\
$\mathrm{CO}_{2}$ & $18.0 \pm 1.0$ & $20.5 \pm 0.2$ & $3.1 \pm 0.1$ & $1.9 \pm 0.9$ \\
$\mathrm{CH}_{4}$ & $4.5 \pm 0.1$ & $3.36 \pm 0.02$ & $0.08 \pm 0.05$ & $0.13 \pm 0.08$ \\
$\mathrm{C}_{2} \mathrm{H}_{4}$ & $1.5 \pm 0.05$ & $0.28 \pm 0.01$ & $83 \%$ & $98.7 \%$ \\
\hline $\mathrm{CO}$ shift conversion & & & $* *$ & $* *$ \\
\hline $\begin{array}{c}\text { Tar content } \\
\left(\mathrm{g} / \mathrm{Nm}^{3}\right)\end{array}$ & 19.5 & $* *$ & & \\
\hline
\end{tabular}

* Gas composition is dry basis (vol-\%) measured by gas chromotography.

** No heavy tar by observation 
gas shift reaction is occurring even at the elevated temperature of the steam reformer. The steam reformer substantially reduced the concentration of $\mathrm{C}_{2} \mathrm{H}_{4}$ but only moderately reduced $\mathrm{CH}_{4}$.

Exiting the high temperature shift reactor, the average gas composition was: 23.7 vol- $\% \mathrm{H}_{2}, 1.4$ vol- $\% \mathrm{CO}, 26.8$ vol- $\% \mathrm{CO}_{2}, 3.1$ vol- $\% \mathrm{CH}_{4}$, and 0.08 vol- $\% \mathrm{C}_{2} \mathrm{H}_{4}$. The high temperature shift reactor reduced $\mathrm{CO}$ content by 7.6 vol- $\%$, representing $83 \%$ conversion. The concentration of $\mathrm{CH}_{4}$ is essentially unchanged through the high temperature shift reactor while the concentration of $\mathrm{C}_{2} \mathrm{H}_{4}$ decreases substantially. The fact that $\mathrm{CH}_{4}$ is essentially unchanged gives some confidence that the high temperature shift reactor was operated with adequate steam. Otherwise, the $\mathrm{Fe}-\mathrm{Cr}$ based catalyst has a tendency to be over-reduced by $\mathrm{H}_{2}$ and $\mathrm{CO}$ to form metallic iron, which catalyzes the methanation reaction:

Eq. 21

$$
\mathrm{CO}+3 \mathrm{H}_{2} \rightarrow \mathrm{CH}_{4}+\mathrm{H}_{2} \mathrm{O}
$$

Exiting the low temperature shift reactor, the average gas composition was: 26.7 vol- $\% \mathrm{H}_{2}, 0.11$ vol- $\% \mathrm{CO}, 27.4$ vol- $\% \mathrm{CO}_{2}, 1.9$ vol- $\% \mathrm{CH}_{4}$, and 0.13 vol- $\% \mathrm{C}_{2} \mathrm{H}_{4}$. The total decrease in $\mathrm{CO}$ content through the shift-reactor system of 8.9 vol- $\%$ represents an overall $\mathrm{CO}$ conversion of $98.7 \%$. The overall increase in $\mathrm{H}_{2}$ due to the combined action of the steam reforming and shift reactors is 18.1 vol-\%. Within the uncertainty of the measurements, the concentrations of $\mathrm{CH}_{4}$ and $\mathrm{C}_{2} \mathrm{H}_{4}$ were not significantly affected through the low temperature shift reactor.

Table 18 investigates the effect of space velocity on performance of the high temperature shift (HTS) reactor was investigated for baseline conditions of $\mathrm{T}_{\mathrm{TR}}=800^{\circ} \mathrm{C}, \mathrm{T}_{\mathrm{HTS}}=350^{\circ} \mathrm{C}$, and steam gas ratio $\mathrm{S} / \mathrm{G}=0.9$. Three flow rates were tested: $2.6,3.8$, and $4.6 \mathrm{~L} / \mathrm{min}$, which represent space velocity varying from 1350 to $2300 \mathrm{~h}^{-1}$. Carbon monoxide content was reduced from 6.7 vol- $\%$ to less than 2.7 vol\% while $\mathrm{H}_{2}$ increased from 17.8 vol-\% to as high as 28.13 vol- $\%$. $\mathrm{CH}_{4}$ and $\mathrm{C}_{2} \mathrm{H}_{4}$ had almost no concentration change through the high temperature reactor.

Table 19 investigates the effect of temperature on performance of the high temperature shift reactor was conducted for baseline conditions of $\mathrm{T}_{\mathrm{TR}}=800^{\circ} \mathrm{C}, \mathrm{S} / \mathrm{G}=0.7, \mathrm{SV}=1950 \mathrm{~h}^{-1}$. Four temperatures were investigated: $360,380,420$ and $440{ }^{\circ} \mathrm{C}$. Carbon monoxide content was reduced from 8.38 vol- $\%$ to less than 2.6 vol- $\%$. The extent of CO conversion was around $80 \%$ independent of temperature. Hydrogen content increased from 19.8 vol- $\%$ to 28 vol- $\%$.

Table 20 investigates the effect of steam/gas ratio on performance of the high temperature shift reactor was conducted under baseline conditions of $\mathrm{T}_{\mathrm{TR}}=800^{\circ} \mathrm{C}, \mathrm{T}_{\mathrm{HTS}}=400^{\circ} \mathrm{C}, \mathrm{SV}=1950 \mathrm{~h}^{-1}$. Four steam/gas ratios were evaluated: $1.09,0.73,0.67$, and 0.6. Since both injected steam and steam generated from gasification of biomass contributed to the total steam, the steam/gas ratio was determined from the amount of steam condensed from the gas exiting the water-gas shift reactor system. Carbon monoxide content was reduced at the outlets of both the tar reactor and the high temperature shift reactor as a result of steam addition. Carbon monoxide in the raw producer gas was reduced from 13.27 vol- $\%$ to as little as 1.02 vol- $\%$ by the combined action of the tar reactor and the high temperature shift reactor with about $60 \%$ of this change arising from the tar reactor. $\mathrm{CO}$ conversions were more than $75 \%$. High levels of steam clearly enhanced the water-gas shift reaction. Steam levels had little effect on $\mathrm{CH}_{4}$ and $\mathrm{C}_{2} \mathrm{H}_{4}$ concentrations. 
Table 21 investigates the effect of space velocity on performance of the low temperature shift reactor was conducted under baseline conditions of $\mathrm{T}_{\mathrm{TR}}=800^{\circ} \mathrm{C}, \mathrm{T}_{\mathrm{HTS}}=400^{\circ} \mathrm{C}, \mathrm{T}_{\mathrm{LTS}}=210^{\circ} \mathrm{C}$, and $\mathrm{S} / \mathrm{G}=0.8$. The effect of volumetric flow rates were determined for flows of 2.0, 3.0 and 4.0 $\mathrm{L} / \mathrm{min}$, representing a range of space velocities between 800 to $1600 \mathrm{~h}^{-1}$. Carbon monoxide concentration dropped dramatically between the exit of the tar reactor and the exit of the low temperature water gas shift reactor. However, space velocity had negligible effect on the $\mathrm{CO}$ concentration exiting the low temperature water gas shift reactor.

Table 22 investigates the effect of temperature on performance of the low temperature shift reactor was conducted under baseline conditions of $\mathrm{T}_{\mathrm{TR}}=800^{\circ} \mathrm{C}, \mathrm{T}_{\mathrm{HTS}}=400^{\circ} \mathrm{C}, \mathrm{S} / \mathrm{G}=0.8, \mathrm{SV}=$ $1200 \mathrm{~h}^{-1}$. Four reactor temperatures were evaluated: $180,200,220$ and $240^{\circ} \mathrm{C}$. Carbon monoxide concentration exiting the low temperature water gas shift reactor was as low as 0.20 vol- $\%$ at $180{ }^{\circ} \mathrm{C}$ increasing to 0.46 vol- $\%$ as the temperature was raised to $240^{\circ} \mathrm{C}$, a result in accordance with an exothermic reaction. Overall $\mathrm{CO}$ conversion of $95 \%$ is possible and $\mathrm{H}_{2}$ concentrations in air-blown producer gas can reach 29.5 vol- $\%$.

Table 23 investigates the effect of steam/gas ratio on performance of the low temperature shift reactor was conducted under baseline conditions of $\mathrm{T}_{\mathrm{TR}}=800^{\circ} \mathrm{C}, \mathrm{T}_{\mathrm{HTS}}=400^{\circ} \mathrm{C}, \mathrm{T}_{\mathrm{LTS}}=210^{\circ} \mathrm{C}$, $\mathrm{SV}=1200 \mathrm{~h}^{-1}$. Four steam/gas ratios were evaluated: $1.20,0.85,0.54$, and 0.32 . The performance of the low temperature shift reactor in reducing $\mathrm{CO}$ in the producer gas was strongly dependent on steam/gas ratio, reaching 0.19 vol- $\%$ CO for steam/gas ratio of 1.2 .

Atomic concentrations (mol-\%) of carbon, oxygen, sulfur, chlorine, and metals found on fresh and spent catalysts as determined by XPS are summarized in Table 24. Figure 53, Figure 54, and Figure 55 illustrate the x-ray photoelectron spectra of carbon, sulfur, and chlorine for the Ni catalyst, $\mathrm{Fe}-\mathrm{Cr}$ catalyst, and $\mathrm{Cu}-\mathrm{Zn}$ catalyst, respectively. Comparison of fresh and spent catalysts indicates that carbon accumulated on all three kinds of catalysts to some extent. Coking was most serious on the $\mathrm{Cu}-\mathrm{Zn}$ catalyst where the relative amount of carbon almost doubled during the gasification test. Coking on the Ni catalyst was also serious, with carbon content increasing by nearly $30 \%$. Accumulation of sulfur of atomic concentration on the catalysts was less than 0.5 mol- $\%$ while chlorine accumulation represented 1.0 to 2.0 mol-\%. Clearly, some hydrogen chloride and hydrogen sulfide in the raw producer gas broke through the guard bed and deposited on the catalysts. Although the reactors did not show any evidence of catalyst deactivation, the design of the guard bed needs to be improved to protect the metal catalysts from these trace contaminants.

Table 25 summarized the specific surface and average pore diameters of catalysts as determined by BET analysis. In all cases, specific surface area of spent catalysts greatly decreased compared to fresh catalysts. The average pore diameter of Ni-based catalyst decreased while it increased for the other two catalysts. Figure 56, Figure 57, and Figure 57 plot pore volume distributions for the three catalysts as determined by BJH adsorption, illustrating that pore volumes changed greatly during the tests. Generally the micropores and mesopores decreased while macropores remained relatively unchanged. These changes are probably the result of both the reduction of catalysts prior to tests as well as from coking, which blocks pores with carbon. 
Table 18 Effect of Space Velocity on Performance of HTS Reactor $\left(\mathrm{T}_{\mathrm{TR}}=800^{\circ} \mathrm{C}, \mathrm{T}_{\mathrm{HTS}}=350^{\circ} \mathrm{C}\right.$, $\mathrm{S} / \mathrm{G}=0.9$ )

\begin{tabular}{|c|r|r|r|r|r|}
\hline & \multirow{2}{*}{ Gas Constituent } & *Exit of Tar & \multicolumn{3}{|c|}{ Exit of High Temperature Shift } \\
& Raw Gas & Steam Reactor & \multicolumn{3}{|c|}{ Reactor } \\
\cline { 3 - 5 } & & & $1350 \mathrm{~h}^{-1}$ & $1950 \mathrm{~h}^{-1}$ & $2300 \mathrm{~h}^{-1}$ \\
\hline & & & & & \\
$\mathrm{H}_{2}($ vol.\%) & 5.84 & 17.83 & 28.13 & 26.60 & 25.13 \\
$\mathrm{CO}($ vol.\%) & 15.77 & 6.73 & 2.13 & 2.66 & 2.70 \\
$\mathrm{CO}_{2}($ vol.\%) & 14.44 & 19.85 & 26.80 & 26.40 & 25.80 \\
$\mathrm{CH}_{4}($ vol.\%) & 4.19 & 3.06 & 3.27 & 3.27 & 3.27 \\
$\mathrm{C}_{2} \mathrm{H}_{4}($ vol.\%) & 1.95 & 0.55 & 0.26 & 0.26 & 0.26 \\
\hline
\end{tabular}

*Previous study showed that space velocity (SV) does not substantially affect the performance of the tar steam reaction. Therefore the measurements on the tar steam reactor were performed at only one $\mathrm{SV}$ of $1950 \mathrm{~h}^{-1}(3.8 \mathrm{~L} / \mathrm{min})$.

Table 19 Effect of Temperature on Performance of HTS Reactor $\left(\mathrm{T}_{\mathrm{TR}}=800^{\circ} \mathrm{C}, \mathrm{S} / \mathrm{G}=0.7\right.$,

\begin{tabular}{|c|c|c|c|c|c|c|}
\hline \multirow[b]{2}{*}{ Gas Constituent } & \multirow[b]{2}{*}{ Raw Gas } & \multirow{2}{*}{$\begin{array}{c}\text { Exit of } \\
\text { Tar Steam } \\
\text { Reactor }\end{array}$} & \multicolumn{4}{|c|}{ Exit of High Temperature Shift Reactor } \\
\hline & & & $360^{\circ} \mathrm{C}$ & $380^{\circ} \mathrm{C}$ & $420^{\circ} \mathrm{C}$ & $440^{\circ} \mathrm{C}$ \\
\hline $\mathrm{H}_{2}($ vol. \%) & 7.74 & 19.85 & 27.78 & 28.48 & 28.3 & 28.14 \\
\hline $\mathrm{CO}(\mathrm{vol} \%$ \%) & 13.27 & 8.38 & 2.58 & 1.14 & 1.21 & 1.38 \\
\hline $\mathrm{CO}_{2}(\mathrm{vol} . \%)$ & 16.69 & 20.71 & 27.40 & 27.80 & 27.82 & 27.72 \\
\hline $\mathrm{CH}_{4}($ vol. $\%)$ & 3.89 & 3.19 & 2.97 & 2.94 & 2.95 & 2.94 \\
\hline $\mathrm{C}_{2} \mathrm{H}_{4}($ vol. $\%)$ & 1.71 & 0.26 & 0.22 & 0.22 & 0.22 & 0.22 \\
\hline
\end{tabular}


Table 20 Effect of Steam/Gas Ratio (S/G) on Performance of HTS Reactor $\left(\mathrm{T}_{\mathrm{TR}}=800^{\circ} \mathrm{C}\right.$, $\left.\mathrm{T}_{\mathrm{HTS}}=400^{\circ} \mathrm{C}, \mathrm{SV}=1950 \mathrm{~h}^{-1}\right)$

\begin{tabular}{|l|l|r|r|r|r|r|r|r|r|}
\hline \multirow{2}{*}{$\begin{array}{l}\text { Gas } \\
\text { Constituent }\end{array}$} & \multirow{3}{*}{ Raw Gas } & \multicolumn{4}{|c|}{ Exit of Tar Reactor } & \multicolumn{4}{c|}{ Exit of High Temperature } \\
& & 1.09 & 0.73 & 0.67 & 0.6 & 1.09 & 0.73 & 0.67 & \multicolumn{1}{c|}{0.6} \\
\cline { 3 - 10 } & & & & & & & & & \\
\hline & & & & & & & & & \\
$\mathrm{H}_{2}$ (vol.\%) & 7.74 & 21.61 & 20.47 & 19.85 & 18.52 & 29.97 & 28.78 & 28.48 & 27.65 \\
$\mathrm{CO}($ vol.\%) & 13.27 & 5.42 & 7.22 & 8.38 & 10.21 & 1.02 & 1.22 & 1.39 & 2.03 \\
$\mathrm{CO}_{2}$ (vol.\%) & 16.69 & 23.14 & 21.60 & 20.70 & 19.30 & 27.63 & 27.54 & 27.56 & 27.58 \\
$\mathrm{CH}_{4}$ (vol.\%) & 3.89 & 3.05 & 3.17 & 3.19 & 3.24 & 2.94 & 2.95 & 2.94 & 2.95 \\
$\mathrm{C}_{2} \mathrm{H}_{4}$ (vol.\%) & 1.71 & 0.19 & 0.28 & 0.26 & 0.30 & 0.21 & 0.21 & 0.21 & 0.21 \\
\hline
\end{tabular}

Table 21 Effect of Space Velocity on Performance of LTS Reactor $\left(\mathrm{T}_{\mathrm{TR}}=800^{\circ} \mathrm{C}\right.$,

\begin{tabular}{|c|r|r|r|r|r|}
\hline \multirow{2}{*}{$\left.\mathrm{T}_{\mathrm{LTS}}=210^{\circ} \mathrm{C}, \mathrm{S} / \mathrm{G}=0.8\right)$} & *Exit of Tar & \multicolumn{3}{|c|}{ Exit of Low Temperature Shift } \\
Gas Constituent & \multirow{2}{*}{ Raw Gas } & $\begin{array}{c}\text { Reactor } \\
\text { Steam Reactor }\end{array}$ & \multicolumn{3}{|c|}{} \\
\cline { 3 - 6 } & & & $800 \mathrm{~h}^{-1}$ & $1200 \mathrm{~h}^{-1}$ & $1600 \mathrm{~h}^{-1}$ \\
\hline & & & & \\
$\mathrm{H}_{2}($ vol.\%) & 6.53 & 20.50 & 36.11 & 29.68 & 29.13 \\
$\mathrm{CO}($ vol.\%) & 14.18 & 8.04 & 0.4 & 0.43 & 0.49 \\
$\mathrm{CO}_{2}$ (vol.\%) & 16.30 & 20.90 & 28.83 & 28.63 & 28.72 \\
$\mathrm{CH}_{4}$ (vol.\%) & 4.04 & 3.17 & 2.97 & 2.97 & 2.97 \\
$\mathrm{C}_{2} \mathrm{H}_{4}($ vol.\%) & 1.77 & 0.21 & 0.28 & 0.28 & 0.28 \\
\hline
\end{tabular}

*Results from Section 4.3 showed that space velocity (SV) does not substantially affect the performance of the tar steam reaction. Therefore the measurements on the tar steam reactor were performed at only one SV of $1200 \mathrm{~h}^{-1}(3.0 \mathrm{~L} / \mathrm{min})$. 
Table 22 Effect of Temperature on Performance of LTS Reactor $\left(\mathrm{T}_{\mathrm{TR}}=800^{\circ} \mathrm{C}, \mathrm{S} / \mathrm{G}=0.8, \mathrm{SV}=1200 \mathrm{~h}^{-1}\right)$

\begin{tabular}{|c|r|r|r|r|r|r|}
\hline \multirow{2}{*}{ Gas Constituent } & \multirow{2}{*}{$\begin{array}{c}\text { Exit of Tar } \\
\text { Steam Reactor }\end{array}$} & \multicolumn{3}{|c|}{ Exit of Low Temperature Shift Reactor } \\
\cline { 4 - 7 } & & & \multicolumn{1}{c|}{$180^{\circ} \mathrm{C}$} & $200^{\circ} \mathrm{C}$ & $220^{\circ} \mathrm{C}$ & \multicolumn{1}{c|}{$240^{\circ} \mathrm{C}$} \\
\hline & & & & & & \\
$\mathrm{H}_{2}$ (vol.\%) & 6.53 & 20.50 & 29.68 & 29.60 & 29.56 & 29.44 \\
$\mathrm{CO}($ vol.\%) & 14.18 & 8.04 & 0.20 & 0.31 & 0.37 & 0.46 \\
$\mathrm{CO}_{2}$ (vol.\%) & 16.30 & 20.90 & 28.50 & 28.45 & 28.51 & 28.30 \\
$\mathrm{CH}_{4}($ vol.\%) & 4.04 & 3.17 & 2.97 & 2.97 & 2.98 & 2.98 \\
$\mathrm{C}_{2} \mathrm{H}_{4}$ (vol.\%) & 1.77 & 0.21 & 0.25 & 0.25 & 0.26 & 0.25 \\
\hline
\end{tabular}

Table 23 Effect of Steam/Gas Ratio (S/G) on Performance of LTS Reactor $\left(\mathrm{T}_{\mathrm{TR}}=800^{\circ} \mathrm{C}\right.$, $\left.\mathrm{T}_{\mathrm{LTS}}=200^{\circ} \mathrm{C}, \mathrm{SV}=1200 \mathrm{~h}^{-1}\right)$

\begin{tabular}{|c|c|c|c|c|c|c|c|c|c|}
\hline \multirow[t]{2}{*}{ Gas Constituent } & \multirow{2}{*}{ Raw Gas } & \multicolumn{4}{|c|}{ Exit of Tar Steam Reactor } & \multicolumn{4}{|c|}{$\begin{array}{c}\text { Exit of Low Temperature } \\
\text { Shift Reactor }\end{array}$} \\
\hline & & 1.2 & 0.85 & 0.54 & 0.32 & 1.2 & 0.85 & 0.54 & 0.32 \\
\hline $\mathrm{H}_{2}($ vol.\%) & 8.81 & 23.86 & 23.53 & 22.80 & 21.07 & 27.83 & 28.1 & 27.75 & 27.51 \\
\hline $\mathrm{CO}(\mathrm{vol} . \%)$ & 13.23 & 7.99 & 9.01 & 10.33 & 12.63 & 0.19 & 0.16 & 0.20 & 0.4 \\
\hline $\mathrm{CO}_{2}(\mathrm{vol} . \%)$ & 17.01 & 20.85 & 20.41 & 19.38 & 17.37 & 27.20 & 27.04 & 26.90 & 26.70 \\
\hline $\mathrm{CH}_{4}(\mathrm{vol} . \%)$ & 3.81 & 3.13 & 3.34 & 3.51 & 3.44 & 3.05 & 3.05 & 3.05 & 3.05 \\
\hline $\mathrm{C}_{2} \mathrm{H}_{4}(\mathrm{vol} . \%)$ & 1.69 & 0.30 & 0.26 & 0.22 & 0.20 & 0.24 & 0.24 & 0.24 & 0.24 \\
\hline
\end{tabular}


Table 24 Concentrations of atomic species in the catalysts as determined by x-ray photoelectron spectroscopy (XPS)

\begin{tabular}{|c|c|c|c|c|c|c|}
\hline & \multicolumn{2}{|c|}{ Ni-based catalyst } & \multicolumn{2}{|c|}{ Fe-Cr based catalyst } & \multicolumn{2}{|c|}{$\mathrm{Cu}-\mathrm{Zn}$ based catalyst } \\
\hline & $\begin{array}{c}\text { Fresh } \\
(\mathrm{mol}-\%)\end{array}$ & $\begin{array}{c}\text { Spent } \\
(\mathrm{mol}-\%)\end{array}$ & $\begin{array}{c}\text { Fresh } \\
(\mathrm{mol}-\%)\end{array}$ & $\begin{array}{c}\text { Spent } \\
(\mathrm{mol}-\%)\end{array}$ & $\begin{array}{c}\text { Fresh } \\
(\mathrm{mol}-\%)\end{array}$ & $\begin{array}{c}\text { Spent } \\
(\mathrm{mol}-\%)\end{array}$ \\
\hline Carbon C1s & 10.16 & 13.14 & 18.27 & 18.76 & 12.29 & 22.91 \\
\hline Oxygen O1s & 59.78 & 54.59 & 49.96 & 48.96 & 46.33 & 48.10 \\
\hline Sulfur S2p & 0.00 & 0.02 & 0.03 & 0.49 & 0.07 & 0.12 \\
\hline Chlorine $\mathrm{Cl} 2 \mathrm{p}$ & 0.29 & 1.09 & 1.00 & 1.23 & 2.12 & 4.49 \\
\hline Aluminum A12p & 14.50 & 15.27 & -- & -- & -- & - \\
\hline Nickel Ni2p & 8.51 & 8.65 & -- & - & -- & - \\
\hline Iron $\mathrm{Fe} 2 \mathrm{p}$ & -- & -- & 27.25 & 23.76 & -- & - \\
\hline Chromium Cr2p & -- & -- & 3.48 & 6.80 & -- & - \\
\hline Copper Cu2p & -- & -- & -- & -- & 13.26 & 4.84 \\
\hline Zinc Zn2p & -- & -- & -- & -- & 25.93 & 19.54 \\
\hline
\end{tabular}

Table 25 BET analysis of catalysts

\begin{tabular}{|c|c|c|c|c|c|c|}
\hline & \multicolumn{2}{|l|}{ Ni-based catalyst } & \multicolumn{2}{l|}{ Fe-Cr based catalyst } & \multicolumn{2}{c|}{ Cu-Zn based catalyst } \\
\cline { 2 - 7 } & Fresh & Spent & Fresh & Spent & Fresh & Spent \\
\hline Specific Surface $\left(\mathrm{m}^{2} / \mathrm{g}\right)$ & 18.09 & 5.66 & 131.12 & 34.3657 & 108.106 & 77.77 \\
\hline Average diameter $(\AA)$ & 128.57 & 113.33 & 61.63 & 191.77 & 59.89 & 83.17 \\
\hline
\end{tabular}



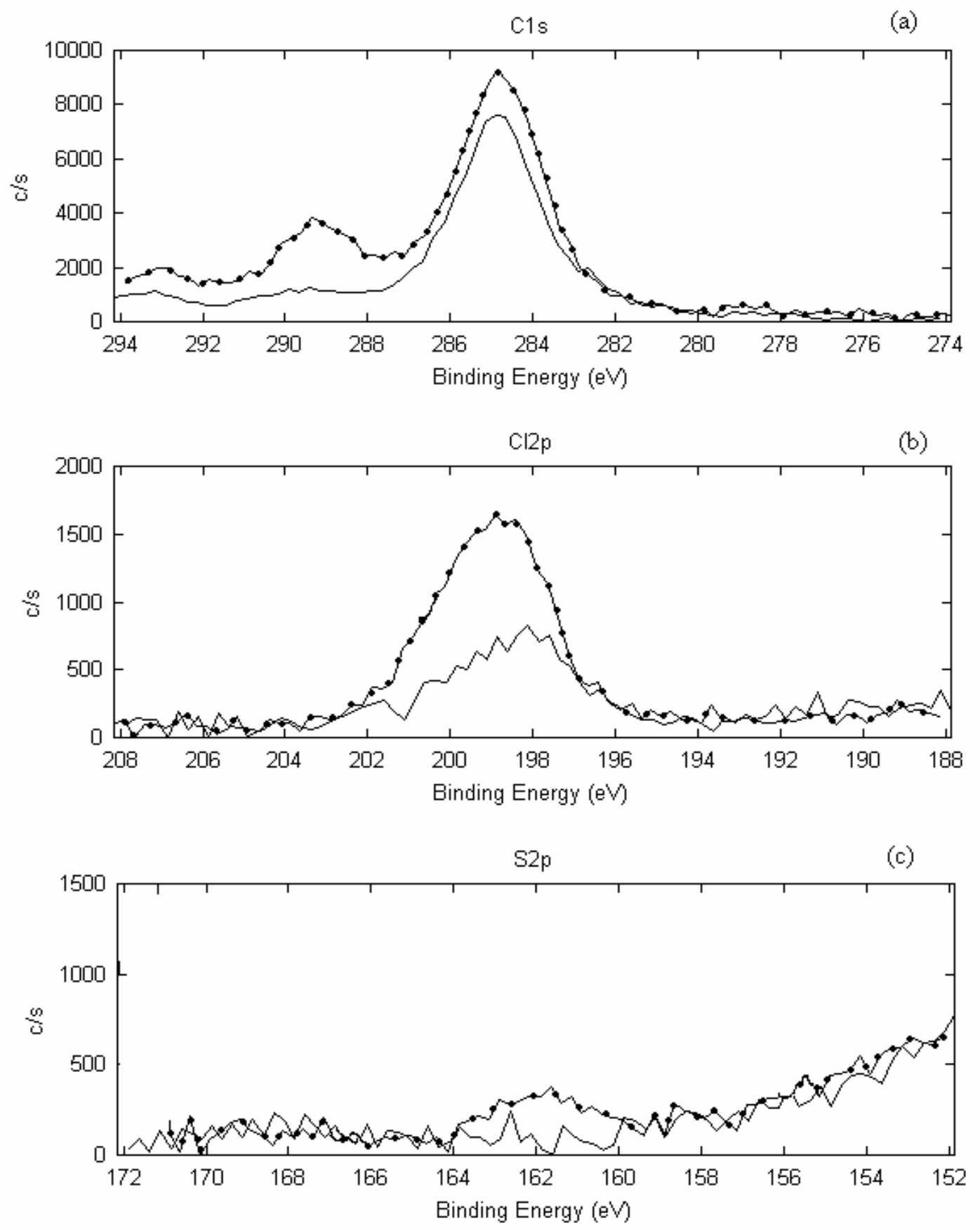

— Fresh Ni-based Catalyst $\longrightarrow$ Used Ni-based catalyst

Figure 53 X-ray photoelectron spectra (XPS) of Ni- based catalysts using Physical Electronics 5500 equipped with Al Ka source (a) Carbon 1s peak, (b) Chlorine 2p peak, (c) Sulfur 2p peak. 

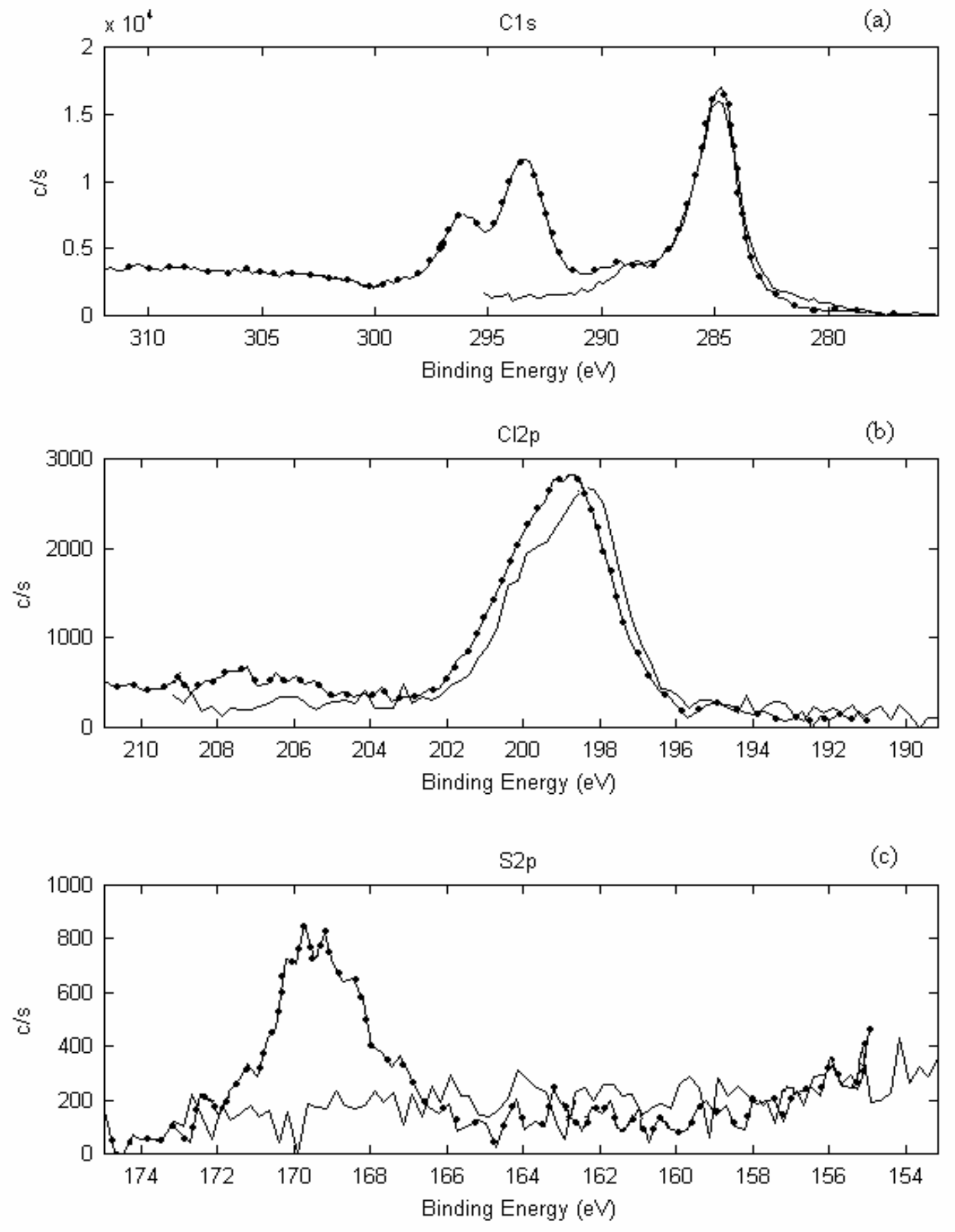

$\longrightarrow$ Fresh Fe-Cr Catalyst $\longrightarrow$ Used Fe-Cr Catalyst

Figure 54 X-ray photoelectron spectra (XPS) of Fe-Cr based catalysts using Physical Electronics 5500 equipped with $\mathrm{Al} \mathrm{K} \alpha$ source (a) Carbon 1s peak, (b) Chlorine $2 \mathrm{p}$ peak, (c) Sulfur $2 \mathrm{p}$ peak. 

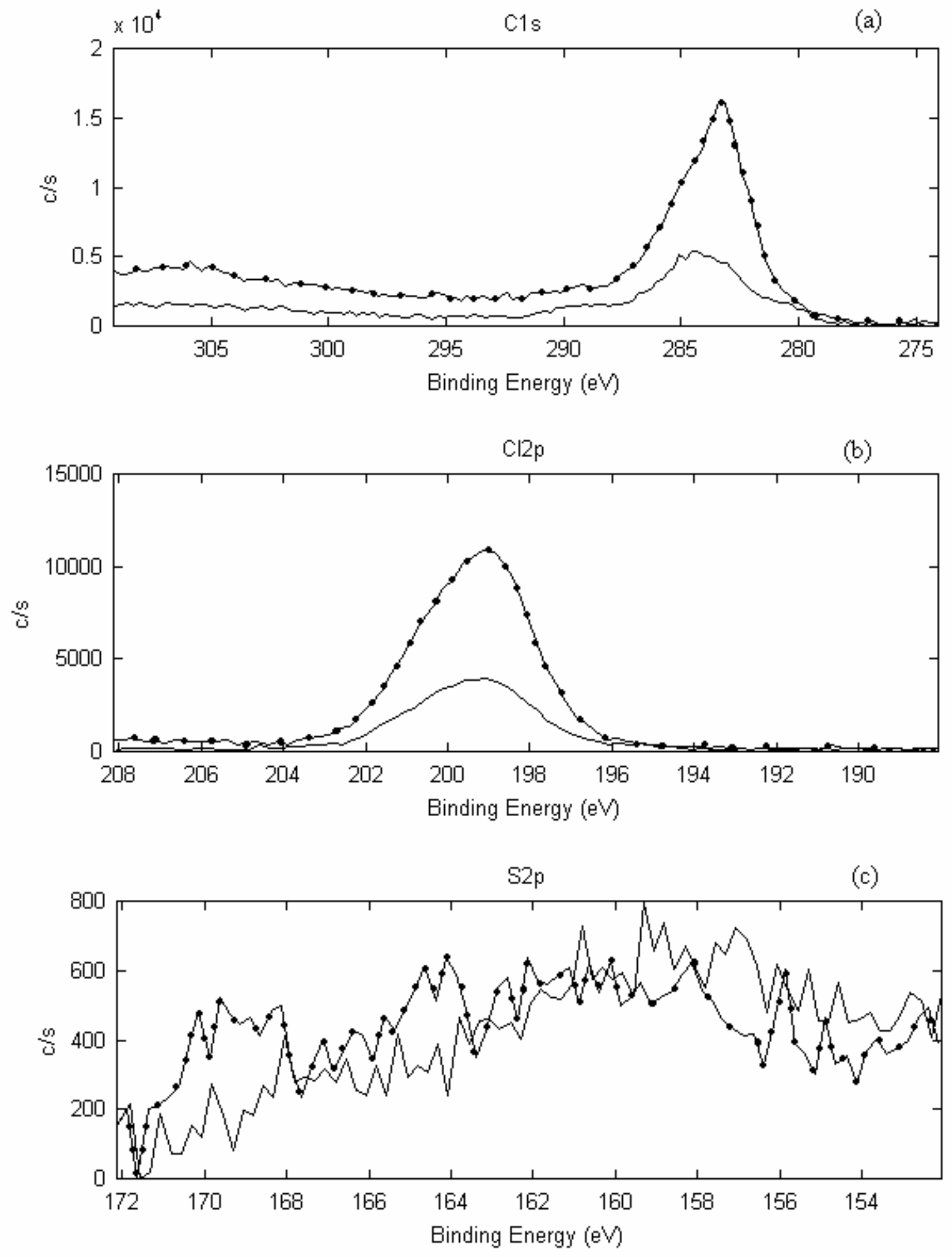

$\longrightarrow$ Fresh Cu-Zn Catalyst $\quad \longrightarrow$ Used Cu-Zn Catalyst

Figure 55 X-ray photoelectron spectroscopy (XPS) of Cu-Zn based catalysts using Physical Electronics 5500 equipped with Al Ka source (a) Carbon 1s peak, (b) Chlorine 2p peak, (c) Sulfur 2p peak. 


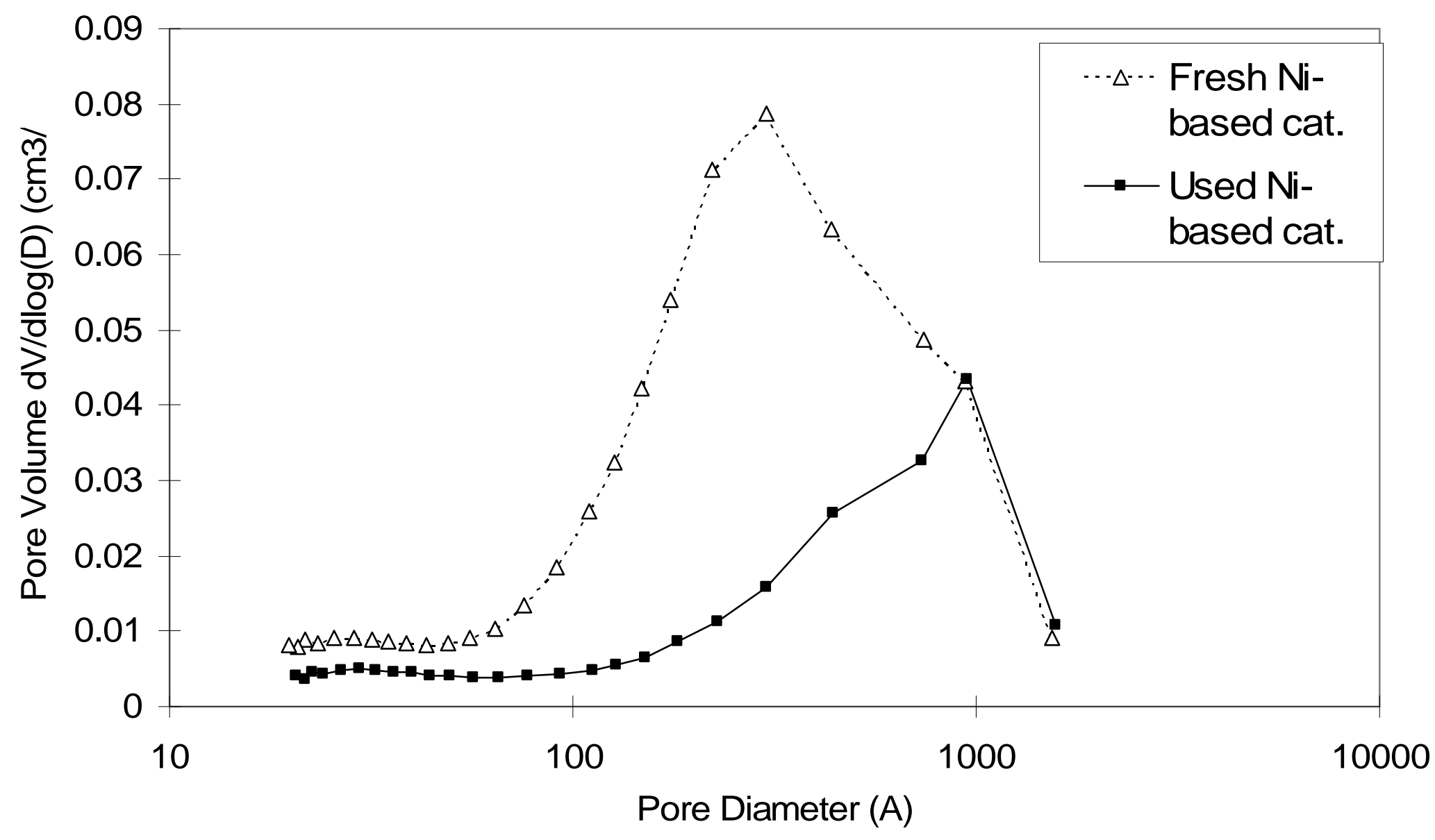

Figure $56 \mathrm{BJH}$ pore volume $\mathrm{dV} / \mathrm{d} \log \mathrm{D}$ versus pore diameter of Ni-based catalyst peak. 


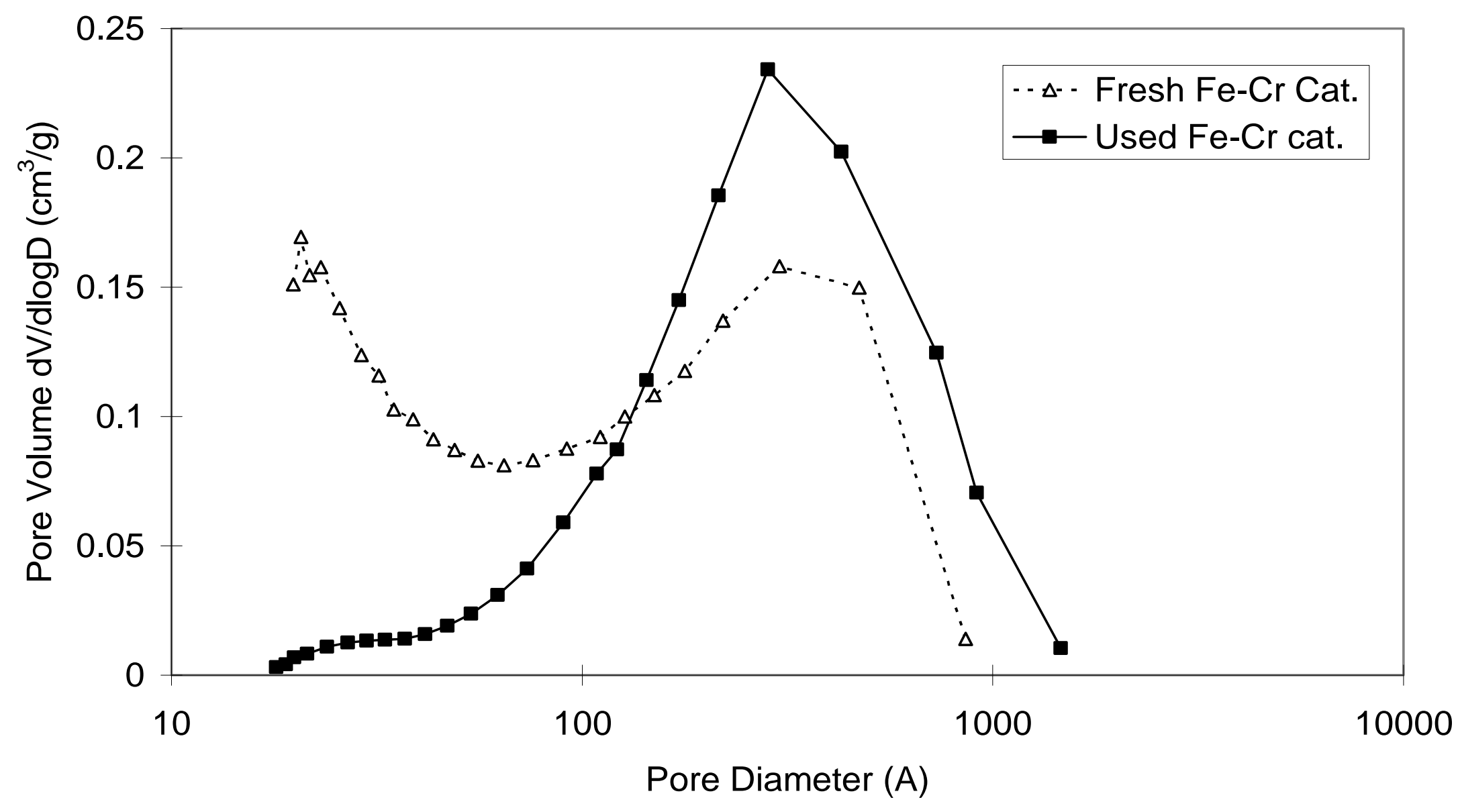

Figure $57 \mathrm{BJH}$ pore volume $\mathrm{dV} / \mathrm{d} \log \mathrm{D}$ versus pore diameter of $\mathrm{Fe}-\mathrm{Cr}$ based catalyst 


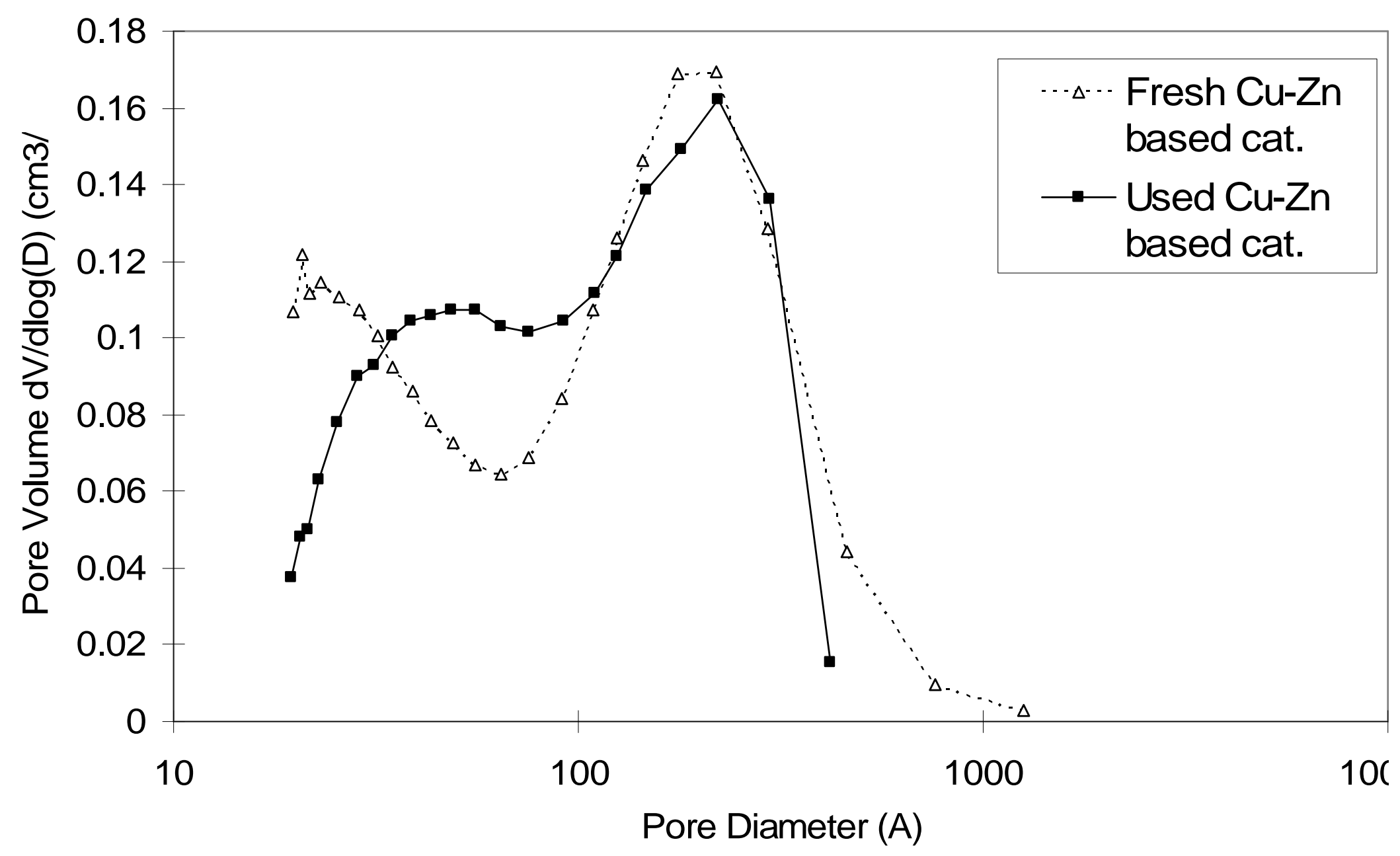

Figure $58 \mathrm{BJH}$ pore volume $\mathrm{dV} / \mathrm{d} \log \mathrm{D}$ versus pore diameter of $\mathrm{Cu}-\mathrm{Zn}$ based catalyst 


\section{Combined Catalyst/Sorbent System}

This task was initiated in the last year of this three year project, after completion of tests of the conventional water-gas shift catalysts. The results are preliminary and have not yet been published.

Numerous batches of core-in-shell pellets were prepared and tested while investigating different combinations of core materials, shell materials and catalysts. This effort has shown that nickel is a very good catalyst for the reaction of steam with $\mathrm{CO}, \mathrm{CH}_{4}$ or toluene (a stand-in for tar). It has also shown that $\mathrm{CaO}$ is a good sorbent whose activity can be stabilized by the presence of $\mathrm{MgO}$ as in dolomitic lime (dolime). In addition, this work has shown that a mixture of $\alpha$-alumina and $\gamma$-alumina provides a higher surface area catalyst support than $\alpha$-alumina alone, although the later produces stronger shells. Furthermore the work has shown that the shells can be strengthened by the addition of lanthanum which is a known stabilizing agent for alumina.

All three of the catalyst/sorbent formulations shown in Table 26 produced good results in performance tests. Dolime was the basic ingredient of the pellet cores in all of the formulations. However since $\mathrm{MgO}$ is not an effective sorbent for $\mathrm{CO}_{2}$ under the intended usage conditions, the absorption capacity of the cores derived from dolime is not as great as those derived from limestone. Therefore, to improve the absorption capacity some limestone was mixed with the dolime in two cases. Although a mixture of $\alpha$-alumina, $\gamma$-alumina and limestone was used for preparing the shells in each case, the proportions were varied among the three cases. Also in the third case some lanthanum was introduced into the shells. After the core-in-shell pellets were formed, they were calcined at $900^{\circ} \mathrm{C}$ for $3 \mathrm{hr}$ to strengthen the shell material by partially sintering it. The pellets were then impregnated with nickel nitrate solution. In two cases the impregnation was repeated which resulted in a higher nickel content. The pellets were subsequently calcined at $600^{\circ} \mathrm{C}$ to convert nickel salt to $\mathrm{NiO}$ and then reduced with $\mathrm{H}_{2}$ at $550^{\circ} \mathrm{C}$ to elemental $\mathrm{Ni}$. The change in weight of a pellet sample during reduction provides the means for determining the reported "reducible nickel content."

The results of several successful performance test achieved with the different pellet formulations are presented in Table 27. These tests were conducted with a small, vertical, tubular reactor which was packed with core-in-shell pellets and heated by a surrounding electric furnace. The reactor feed consisted of a mixture of steam and one or more of the carbon containing reactants in a 3:1 mole ratio of $\mathrm{H}_{2} \mathrm{O}: \mathrm{C}$. The product gas stream was analyzed by gas chromatography and the results are indicated in Table 27 on a dry basis. Before each performance test the sorbent was regenerated by heating the bed to $750^{\circ} \mathrm{C}$ in a stream of $\mathrm{N}_{2}$ and then the catalyst was reduced with $\mathrm{H}_{2}$ at $550^{\circ} \mathrm{C}$. The desired temperature for the test was set and the feed mixture was supplied. During the early part of the test almost all of the $\mathrm{CO}_{2}$ was absorbed as indicated by the gas composition reported for the " $\mathrm{CO}_{2}$ absorbed" operating mode. When the sorbent became saturated, $\mathrm{CO}_{2}$ was no longer absorbed which was reflected by the gas composition indicated by the "no absorption" operating mode. A comparison of the gas composition for the two operating modes for any give set of operating conditions shows that in every test $\mathrm{CO}_{2}$ absorption had a large effect on the resulting gas composition. While $\mathrm{CO}_{2}$ was being absorbed the $\mathrm{H}_{2}$ concentration was always in excess of $90 \mathrm{~mol} \%$ and the concentrations of both $\mathrm{CO}_{2}$ and $\mathrm{CO}$ were low. On the other hand, when $\mathrm{CO}_{2}$ was not being absorbed, the concentration of $\mathrm{H}_{2}$ was in the 60 to $70 \mathrm{~mol} \%$ range because of the presence of significant concentrations of $\mathrm{CO}, \mathrm{CO}_{2}$ and 
Table 26 Different formulations used in preparing core-in-shell pellets and the reducible nickel content of the finished pellets.

\begin{tabular}{|c|c|c|c|c|c|c|}
\hline \multirow{2}{*}{$\begin{array}{l}\text { Formulation } \\
\text { No. }\end{array}$} & \multicolumn{2}{|c|}{ Core composition } & \multicolumn{2}{|c|}{ Shell composition } & \multicolumn{2}{|c|}{ Nickel content } \\
\hline & Material & wt.\% & Material & wt. $\%$ & Treatments $^{\mathrm{a}}$ & wt. $\%$ \\
\hline $\mathrm{I}$ & $\begin{array}{l}\text { limestone } \\
\text { dolime }\end{array}$ & $\begin{array}{l}50 \\
50\end{array}$ & $\begin{array}{l}\alpha \text {-alumina } \\
\gamma \text {-alumina } \\
\text { limestone }\end{array}$ & $\begin{array}{l}64 \\
16 \\
20\end{array}$ & twice & 6 \\
\hline II & dolime & 100 & $\begin{array}{l}\alpha \text {-alumina } \\
\gamma \text {-alumina } \\
\text { limestone }\end{array}$ & $\begin{array}{l}66.5 \\
28.5 \\
5.0\end{array}$ & once & 3 \\
\hline $\mathrm{III}^{\mathrm{b}}$ & $\begin{array}{l}\text { limestone } \\
\text { dolime }\end{array}$ & $\begin{array}{l}50 \\
50\end{array}$ & $\begin{array}{l}\alpha \text {-alumina } \\
\gamma \text {-alumina } \\
\text { limestone }\end{array}$ & $\begin{array}{l}76 \\
19 \\
5\end{array}$ & twice & 5 \\
\hline $\begin{array}{l}{ }^{\mathrm{a}} \text { Number of } \\
{ }^{\mathrm{b}} \text { Also contai }\end{array}$ & ums & & & & & \\
\hline
\end{tabular}


Table 27 Results of reacting steam with various carbon compounds using a 3:1 mole ratio of steam to carbon and 1 atm total pressure, with and without $\mathrm{CO}_{2}$ absorption.

\begin{tabular}{|c|c|c|c|c|c|c|c|c|c|}
\hline \multirow{2}{*}{$\begin{array}{l}\text { Pellet } \\
\text { formulation }\end{array}$} & \multicolumn{3}{|c|}{ Feed composition } & \multirow{2}{*}{$\begin{array}{l}\text { Temp. } \\
{ }^{\circ} \mathrm{C}\end{array}$} & \multirow{2}{*}{$\begin{array}{l}\text { Operating } \\
\text { mode }\end{array}$} & \multicolumn{4}{|c|}{ Product gas composition, $\mathrm{mol} \% \mathrm{a}$} \\
\hline & Gas & $\mathrm{mol} \%$ & $\mathrm{mmol} / \mathrm{h}$ & & & $\mathrm{H}_{2}$ & $\mathrm{CO}$ & $\mathrm{CO}_{2}$ & $\mathrm{CH}_{4}$ \\
\hline $\begin{array}{l}\text { I } \\
(6 \% \mathrm{Ni})\end{array}$ & $\mathrm{CH}_{4}$ & 100 & 36.4 & 575 & $\begin{array}{l}\mathrm{CO}_{2} \text { absorb. } \\
\text { No absorb. }\end{array}$ & $\begin{array}{l}95 \\
71\end{array}$ & $\begin{array}{l}1 \\
6\end{array}$ & $\begin{array}{l}1 \\
14\end{array}$ & $\begin{array}{l}3 \\
9\end{array}$ \\
\hline $\begin{array}{l}\text { II } \\
(3 \% \mathrm{Ni})\end{array}$ & $\mathrm{CO}$ & 100 & 36.4 & 550 & $\begin{array}{l}\mathrm{CO}_{2} \text { absorb. } \\
\text { No absorb. }\end{array}$ & $\begin{array}{l}96 \\
59\end{array}$ & $\begin{array}{l}1 \\
3\end{array}$ & $\begin{array}{l}3 \\
38\end{array}$ & $\begin{array}{l}0 \\
0\end{array}$ \\
\hline $\begin{array}{l}\text { III }^{\mathrm{b}} \\
(5 \% \mathrm{Ni})\end{array}$ & $\mathrm{C}_{7} \mathrm{H}_{8}$ & 100 & 4.6 & 550 & $\begin{array}{l}\mathrm{CO}_{2} \text { absorb. } \\
\text { No absorb. }\end{array}$ & $\begin{array}{l}96 \\
66\end{array}$ & $\begin{array}{l}3 \\
10\end{array}$ & $\begin{array}{l}1 \\
20\end{array}$ & $\begin{array}{l}0 \\
4\end{array}$ \\
\hline $\begin{array}{l}\mathrm{III}^{\mathrm{b}} \\
(5 \% \mathrm{Ni})\end{array}$ & $\begin{array}{l}\mathrm{CO} \\
\mathrm{C}_{7} \mathrm{H}_{8}\end{array}$ & $\begin{array}{l}80 \\
20\end{array}$ & $\begin{array}{l}18.2 \\
4.6\end{array}$ & 550 & $\begin{array}{l}\mathrm{CO}_{2} \text { absorb. } \\
\text { No absorb. }\end{array}$ & $\begin{array}{l}95 \\
63\end{array}$ & $\begin{array}{l}1 \\
6\end{array}$ & $\begin{array}{l}1 \\
23\end{array}$ & $\begin{array}{l}3 \\
8\end{array}$ \\
\hline $\begin{array}{l}\text { I } \\
(6 \% \mathrm{Ni})\end{array}$ & $\begin{array}{l}\mathrm{CO} \\
\mathrm{CH}_{4} \\
\mathrm{C}_{7} \mathrm{H}_{8}\end{array}$ & $\begin{array}{l}47 \\
47 \\
6\end{array}$ & $\begin{array}{l}18.2 \\
18.2 \\
2.3\end{array}$ & 575 & $\begin{array}{l}\mathrm{CO}_{2} \text { absorb. } \\
\text { No absorb. }\end{array}$ & $\begin{array}{l}91 \\
62\end{array}$ & $\begin{array}{l}1 \\
10\end{array}$ & $\begin{array}{l}2.5 \\
21\end{array}$ & $\begin{array}{l}5.5 \\
7\end{array}$ \\
\hline
\end{tabular}

${ }^{\mathrm{a}}$ Reported on a dry basis

${ }^{\mathrm{b}}$ Also contained lanthanum 
sometimes $\mathrm{CH}_{4}$. The results also indicate that the catalyst was effective in promoting the reaction of steam with each of the three other reactants.

Following the test with steam and toluene some carbon was found deposited on the surface of the pellets and on the wall of the reactor above the bed indicating thermal decomposition of the toluene. This problem may be solved by increasing the steam to carbon ratio or lowering the operating temperature.

These results are highly encouraging because they show that it should be possible to upgrade producer gas in a single step to a product which is nearly pure hydrogen by employing a bed of material that combines a catalyst and sorbent in a single pellet. Further development of the material is required to provide a material which is highly stable and can be regenerated repeatedly and will last a long time. This will require optimizing the composition and preparation conditions for the material.

\subsection{Measurements of Ammonia and Sulfur}

$\mathrm{NH}_{3}$ and $\mathrm{H}_{2} \mathrm{~S}$ concentrations were determined in raw syngas from the biomass gasifier during three days of gasifying seed corn. In addition, $\mathrm{NH}_{3}$ concentrations in raw syngas were determined during a single day of gasifying switchgrass. Typical nitrogen contents (the primary factor affecting $\mathrm{NH}_{3}$ concentrations in the syngas) for the corn and switchgrass were $1 \%$ and $0.5 \%$ (as-received basis), respectively. A summary of the biomass gasification tests is provided in Table 28. The $\mathrm{NH}_{3}$ was collected in dilute acid impingers and then analyzed titrimetrically as described earlier in this report, while the $\mathrm{H}_{2} \mathrm{~S}$ was determined using Drager tubes. $\mathrm{No} \mathrm{H}_{2} \mathrm{~S}$ sampling was performed while gasifying switchgrass.

Results of the $\mathrm{NH}_{3}$ and $\mathrm{H}_{2} \mathrm{~S}$ determinations are summarized in Table 29, which provides data on the range, average, standard deviation, and coefficient of variance (COV) observed for the $\mathrm{NH}_{3}$ and $\mathrm{H}_{2} \mathrm{~S}$ concentrations determined in each test series. As noted earlier, no $\mathrm{H}_{2} \mathrm{~S}$ sampling was performed during switchgrass gasification, so corresponding entries for $\mathrm{H}_{2} \mathrm{~S}$ in that table are left blank.

For Test Series 1, the two $\mathrm{NH}_{3}$ samples were collected back-to-back. In other words, when the first sampling was finished, the second sampling was started immediately. Results from the impinger analyses gave an average $\mathrm{NH}_{3}$ concentration in the syngas of $5458 \mu \mathrm{g} / \mathrm{L}$, with a COV of only $2 \%$. Thus, excellent repeatability was obtained for $\mathrm{NH}_{3}$ concentrations in this test series. It should also be noted that aliquots of some of the impinger solutions from this test series were spiked with known amounts of $\mathrm{NH}_{3}$. Analytical results indicated that there was full (95-100\%) recovery of the analytical spikes, indicating that there are no significant analytical matrix effects, even though the impinger solutions are moderately colored after sampling. Results of the impinger analyses also showed that $98 \%$ of the $\mathrm{NH}_{3}$ was collected in the first impinger of each impinger set.

The $\mathrm{H}_{2} \mathrm{~S}$ readings in Test Series 1 were taken at 5, 30, 45, and 240 minutes into the gasification run. The $\mathrm{H}_{2} \mathrm{~S}$ results from this test series also showed good reproducibility, having an average 
value of about $200 \mathrm{ppm}$ and a COV of less than $10 \% . \mathrm{H}_{2} \mathrm{~S}$ concentrations did not show any correlation with the amount of time into the gasification run.

In Test Series 2, four $\mathrm{NH}_{3}$ samples were collected over a period of three hours and were spaced evenly over that time frame. The first two samples used $\mathrm{H}_{2} \mathrm{SO}_{4}$ absorbing solutions, while the last two samples used $\mathrm{HCl}$ absorbing solutions. Results of the analyses indicated that the $\mathrm{NH}_{3}$ concentrations were slightly lower than those obtained in Test Series 1. The tests conducted in Test Series 1 used a different dry gas meter than in Test Series 2 or 3. Therefore, gas meter calibration issues might come into play. Also, differences in $\mathrm{NH}_{3}$ levels observed between different days of testing with corn might be related to gasifier operating parameters or variations in the nitrogen content of the corn. The $\mathrm{NH}_{3}$ results from the four individual samples collected in Test Series 2 are presented in Table 30 in order to show the comparison between using $\mathrm{HCl}$ and $\mathrm{H}_{2} \mathrm{SO}_{4}$ absorbing solutions. Those results are presented in the same order that the samples were collected. The average $\mathrm{NH}_{3}$ results from the $\mathrm{HCl}$ solutions were slightly (15\%) higher than those from the $\mathrm{H}_{2} \mathrm{SO}_{4}$ solutions. However, one of the $\mathrm{NH}_{3}$ concentration values obtained while using $\mathrm{H}_{2} \mathrm{SO}_{4}$ was significantly lower than the other values and appears to be an outlier. If that outlier were removed, then the results obtained while using $\mathrm{HCl}$ would only be $8 \%$ higher than the remaining value obtained while using $\mathrm{H}_{2} \mathrm{SO}_{4}$. It does not appear that the type of acid used affects results significantly. However, in view of the limited statistics, additional study is advised before a definitive conclusion can be drawn. Even when the low value of $3309 \mu \mathrm{g} / \mathrm{L}$ is included in the overall average, the COV is only $9 \%$, which is still good precision under field sampling conditions.

The $\mathrm{H}_{2} \mathrm{~S}$ readings in Test Series 2 were collected at 45, 75, and 180 minutes into the gasification run and showed no correlation with time. The average $\mathrm{H}_{2} \mathrm{~S}$ value from this test series was in excellent agreement with the average $\mathrm{H}_{2} \mathrm{~S}$ value from Test Series 1 .

For Test Series 3, only $\mathrm{HCl}$ absorbing solutions were used. However, two different $\mathrm{HCl}$ solution volumes $(100 \mathrm{~mL}$ or $200 \mathrm{~mL})$ per impinger were tested to help determine whether or not $100 \mathrm{~mL}$ of absorbing solution would still effectively capture the $\mathrm{NH}_{3}$. The $\mathrm{NH}_{3}$ results obtained from the impinger analyses are shown below in Table 31. Using 100-mL volumes of absorbing solutions seemed to give results comparable (within 10\%) to those obtained when using $200-\mathrm{mL}$ volumes. The first $\mathrm{NH}_{3}$ value seems to be somewhat of an outlier. If that value were excluded, then the results obtained with $100 \mathrm{~mL}$ of absorbing solution would be within $5 \%$ of the results obtained with $200 \mathrm{~mL}$ of absorbing solution. If only $100 \mathrm{~mL}$ of the absorbing solution are needed, then the two impinger catches can be combined to give quantitative recovery of $\mathrm{NH}_{3}$ while greatly reducing sample processing time, impinger preparation time, and analytical costs. It should also be noted that, as with previous tests, at least $98 \%$ of the total $\mathrm{NH}_{3}$ collected was captured in the first acid impinger in each test. This is important because it suggests an approach for greatly reducing sample preparation time. If it is assumed that $98 \%$ of the $\mathrm{NH}_{3}$ is always captured in the first impinger under a broad range of syngas compositions, and if a $2 \%$ loss is considered to be inconsequential, then only the solution in the first impinger needs to be routinely analyzed. This would cut sample processing time in half, reduce analytical costs, and reduce the time necessary to prepare impinger solutions for sampling. 
Table 28 Summary of biomass gasification tests

\begin{tabular}{lllccc}
\hline $\begin{array}{l}\text { Test } \\
\text { Series }\end{array}$ & Feedstock & Absorbing Solution & $\mathrm{H}_{2}$ of $\frac{\text { S Samples }}{3} /$ & $\begin{array}{c}\text { Duration of } \\
\text { Gasification }(\mathrm{hrs})\end{array}$ & $\begin{array}{c}\text { Temp. of } \\
\text { Bed }\left({ }^{\circ} \mathrm{C}\right)\end{array}$ \\
\hline 1 & Corn & $5 \% \mathrm{H}_{2} \mathrm{SO}_{4}$ & $2 / 4$ & 4 & 735 \\
2 & Corn & $5 \% \mathrm{H}_{2} \mathrm{SO}_{4}$ and HCl & $4 / 3$ & 3 & 735 \\
3 & Corn & $5 \% \mathrm{HCl}^{\mathrm{b}}$ & $4 / 5$ & 3 & 790 \\
4 & Switchgrass & $5 \% \mathrm{HCl}$ & $4 / 0$ & 4 & 735 \\
\hline
\end{tabular}

${ }^{a}$ The number of $\mathrm{NH}_{3}$ samples (15-30 minutes each) and $\mathrm{H}_{2} \mathrm{~S}$ readings collected over the duration of the gasification run.

${ }^{b}$ Some of the samples in this test series were collected using $\mathrm{H}_{2} \mathrm{SO}_{4}$ impingers, and some samples were collected using $\mathrm{HCl}$ impingers for comparative purposes.

Table $29 \mathrm{NH}_{3}$ and $\mathrm{H}_{2} \mathrm{~S}$ concentrations in raw syngas from biomass gasification

\begin{tabular}{|c|c|c|c|c|c|c|}
\hline \multirow{2}{*}{$\begin{array}{l}\text { Test } \\
\text { Series }\end{array}$} & \multicolumn{3}{|c|}{$\mathrm{NH}_{3}$ Conc. $(\mu \mathrm{g} / \mathrm{L})$} & \multicolumn{3}{|c|}{$\mathrm{H}_{2} \underline{S}$ S Conc. (ppm) } \\
\hline & Range & Ave. $+/-\sigma$ & $\mathrm{COV}(\%)$ & Range & Ave. $+/-\sigma$ & $\operatorname{COV}(\%)$ \\
\hline $1^{\mathrm{a}}$ & $5374-5542$ & $\overline{5458 \pm 119}$ & 2 & $190-225$ & $205 \pm 15$ & 7 \\
\hline $2^{b}$ & $3309-4117$ & $3775 \pm 351$ & 9 & $180-230$ & $207 \pm 25$ & 12 \\
\hline $3^{c}$ & $3132-3645$ & $3330 \pm 223$ & 7 & $230-340$ & $260 \pm 46$ & 18 \\
\hline $3^{\mathrm{d}}$ & $3132-3321$ & $3226 \pm 94$ & 3 & $230-260$ & $240 \pm 14$ & 6 \\
\hline $4^{\mathrm{e}}$ & $816-2191$ & $1422 \pm 569$ & 40 & ----- & ----- & --- \\
\hline
\end{tabular}

${ }^{\mathrm{a}} \mathrm{NH}_{3}$ sampled twice over a 4-hr period; $\mathrm{H}_{2} \mathrm{~S}$ sampled four times throughout the 4-hr period.

${ }^{\text {b }} \mathrm{NH}_{3}$ sampled four times over a 3-hr period; $\mathrm{H}_{2} \mathrm{~S}$ sampled three times over a 2-hr period.

${ }^{\mathrm{c}} \mathrm{NH}_{3}$ and $\mathrm{H}_{2} \mathrm{~S}$ each sampled 4-5 times over a 3-hour period.

${ }^{d}$ Results recalculated after removing a high outlier in both the $\mathrm{NH}_{3}$ and $\mathrm{H}_{2} \mathrm{~S}$ results.

e $\mathrm{NH}_{3}$ sampled four times over a 4-hr period.

Table 30 Results of individual $\mathrm{NH}_{3}$ determinations in Test Series 2

\begin{tabular}{cc}
\hline Absorbing Solution & $\mathrm{NH}_{3}$ Concentration in Syngas $(\mu \mathrm{g} / \mathrm{L}$, at STP $)$ \\
\hline $\mathrm{H}_{2} \mathrm{SO}_{4}$, test 1 & 3721 \\
$\mathrm{H}_{2} \mathrm{SO}_{4}$, test 2 & 3309 \\
$\mathrm{HCl}_{\text {, test } 1}$ & 4117 \\
$\mathrm{HCl}$, test 2 & 3955 \\
\hline
\end{tabular}


Table 31 Results of $\mathrm{NH}_{3}$ determinations in Test Series 3

\begin{tabular}{cc}
\hline $\begin{array}{c}\text { Solution Volume }(\mathrm{mL}) \\
\text { per Impinger }\end{array}$ & $\begin{array}{c}\mathrm{NH}_{3} \text { Concentration } \\
\text { in Syngas }(\mu \mathrm{g} / \mathrm{L} \text { at STP })\end{array}$ \\
\hline 200 & 3645 \\
200 & 3224 \\
200 & 3321 \\
100 & 3132 \\
\hline
\end{tabular}

The average $\mathrm{NH}_{3}$ concentration was $3330 \pm 223 \mu \mathrm{g} / \mathrm{L}$. The COV was less than $7 \%$, which is excellent under field sampling conditions. As can be seen, the first data point for the $\mathrm{NH}_{3} \mathrm{was}$ slightly higher than the other data points. It is possible that the gas stream was still approaching steady state while collecting the first sample. However, additional work is needed to determine more conclusively the reason for the trend that was observed. If only the last three $\mathrm{NH}_{3}$ data points are used, which is where the readings appear to have reached steady state, the average concentration is $3226 \pm 94 \mu \mathrm{g} / \mathrm{L}$. In that case, the RSD is only $3 \%$.

The $\mathrm{H}_{2} \mathrm{~S}$ readings were taken every 30-60 minutes. Individual $\mathrm{H}_{2} \mathrm{~S}$ readings (in chronological order over a period of about 3 hours) were 340, 260, 230, 240, and $230 \mathrm{ppm}_{\mathrm{v}}$. The last four readings correspond to the time frame associated with the $\mathrm{NH}_{3}$ sampling.

In Test Series 4 (using switchgrass rather than corn), the primary cyclone on the gasifier became plugged. Consequently, the ash content of the syngas stream was much higher than usual and was visually estimated to be at least 10 times the normal loading over the sampling time frame involved. Results of the $\mathrm{NH}_{3}$ sampling indicated that the $\mathrm{NH}_{3}$ concentrations decreased substantially with time. The measured $\mathrm{NH}_{3}$ concentration at the beginning of the switchgrass gasification run was $2190 \mu \mathrm{g} \mathrm{NH} / \mathrm{L}$. Over the next four hours of testing, the $\mathrm{NH}_{3}$ concentration decreased to only $820 \mu \mathrm{g} \mathrm{NH} / \mathrm{L}$. Although the $\mathrm{NH}_{3}$ concentration decreased substantially with time, the data are believed to be accurate for the syngas stream at the sampling point. This large downward trend in the $\mathrm{NH}_{3}$ concentration as a function of time is abnormal and is believed to be related to the unusually high ash loading (which increased with time) in the thimble filter. Specifically, we suspect that the $\mathrm{NH}_{3}$ was interacting with the ash, and that the $\mathrm{NH}_{3}$ levels decreased as the ash loading continued to increase. If increased ash loadings are indeed the reason for decreased $\mathrm{NH}_{3}$ concentrations, it is not known whether the $\mathrm{NH}_{3}$ is adsorbed on the ash or chemically reacting with it. During sampling, the color of the impinger solutions was observed to become progressively lighter with each test, which was the first indicator that something atypical was occurring during sample collection. These results suggest that accurate $\mathrm{NH}_{3}$ determinations may require low ash loadings in the filter. Although the effect needs additional investigation, periodic filter replacement can mitigate this potential problem.

\subsection{Sorbent testing}

Two metal-based sorbents were tested for biomass-derived syngas desulfurization in a fixed bed reactor $(\mathrm{FBR})$. The first sorbent tested was pure zinc oxide $(\mathrm{ZnO})$ particles provided by Zochem of Toronto, Ontario, Canada. The $1 \mathrm{~mm}$ particles were greater than $99.9 \%$ pure $\mathrm{ZnO}$ and had a 
surface area of $3.5 \mathrm{~m}^{2 /} \mathrm{g}$, which was determined by a BET surface area test. The Gas Technology Institute of Des Plaines, Illinois provided the second sorbent, which was a mixed-metal oxide (MMO) sorbent composed of manganese oxide with an aluminum oxide binder. This pelletized sorbent had a BET surface area of $9.2 \mathrm{~m}^{2} / \mathrm{g}$ and the particles sizes ranged between 2 and $3 \mathrm{~mm}$. The syngas concentration for all FBR tests averaged 7 vol- $\% \mathrm{H}_{2}, 51$ vol- $\% \mathrm{~N}_{2}, 12$ vol- $\% \mathrm{CO}$, and 17 vol- $\% \mathrm{CO}_{2}$ on a dry-gas basis. The syngas concentrations deviated at most $2 \%$ from these levels, which would not have effected the operation of the sorbents.

The first FBR experiment performed, FBR01, had no sorbent in the FBR, but rather contained non-reactive $1 \mathrm{~mm}$ glass beads with a bed depth of $6.1 \mathrm{~cm}$ (2 in.). A baseline $\mathrm{H}_{2} \mathrm{~S}$ of $243 \mathrm{ppm}$ was established, then gas was redirected through the FBR where the $\mathrm{H}_{2} \mathrm{~S}$ measurement averaged $238 \mathrm{ppm}$. After 30 minutes of sampling through the FBR, a post-test baseline of $240 \mathrm{ppm}$ was recorded. This test provided confidence in the ability of the empty FBR to operate leak-free without significantly affecting the $\mathrm{H}_{2} \mathrm{~S}$ concentration.

\section{$\underline{\mathrm{ZnO} \text { sorbent desulfurization }}$}

The first test performed with the $\mathrm{ZnO}$ particles, FBR02, was a shakedown run where the sample line was setup and operated under conditions replicating syngas desulfurization conditions. However, $200 \mathrm{ppm} \mathrm{H}_{2} \mathrm{~S}$ in $\mathrm{N}_{2}$ was used instead of real syngas for this evaluation. After three baseline measurements averaging $193 \mathrm{ppm}_{2} \mathrm{~S}$ were recorded; the syngas was redirected to the FBR. The FBR contained $63.26 \mathrm{~g}$ of $\mathrm{ZnO}$, had a bed depth of $6.35 \mathrm{~cm}(2.5 \mathrm{in}$.), and was operated at $450^{\circ} \mathrm{C}\left(842^{\circ} \mathrm{F}\right)$. Two gas sampling rates were sent through the FBR, 0.5 and 1.0 standard liter per minute (slpm). Residence time (adjusted for bed voidage, $\varepsilon_{\mathrm{m}}$ ) was calculated according to:

Eq. $22 \quad \mathrm{t}_{\text {res }}=\frac{\mathrm{V}_{\text {reactor }} * \varepsilon_{\mathrm{m}}}{\mathrm{Q}_{\text {sample }}}$

According to the is equation, residence time is 0.57 seconds for $0.5 \mathrm{slpm}$ and 0.29 seconds for $1.0 \mathrm{slpm}$.

Table 32 illustrates that $\mathrm{ZnO}$ is effective in removing $\mathrm{H}_{2} \mathrm{~S}$ from simulated syngas. At the 0.5 slpm sample rate no $\mathrm{H}_{2} \mathrm{~S}$ was measured while at $1.0 \mathrm{slpm}$ only $2 \mathrm{ppm}$ of $\mathrm{H}_{2} \mathrm{~S}$ was recorded.

Another experiment, FBR08, tested the ability of $\mathrm{ZnO}$ to remove $\mathrm{H}_{2} \mathrm{~S}$ from biomass-derived syngas. For this experiment the FBR was operated at $450^{\circ} \mathrm{C}$ with a bed depth of $6.35 \mathrm{~cm}$ with a bed weight of $62.00 \mathrm{~g}$. Results from this experiment are found in Table 33. The $\mathrm{ZnO}$ particles were able to reduce $\mathrm{H}_{2} \mathrm{~S}$ in a real syngas stream from about $300 \mathrm{ppm}$ to $13 \mathrm{ppm}$ for a gas flow rate of $1.0 \mathrm{slpm}$. Doubling the gas sample rate halved the gas residence time, resulting in an outlet concentration for $\mathrm{H}_{2} \mathrm{~S}$ of $17 \mathrm{ppm}$. Further increasing the gas flow rate to $2.25 \mathrm{slpm}$ increased $\mathrm{H}_{2} \mathrm{~S}$ to $20 \mathrm{ppm}$. After these measurements were performed, the gas flow rate was reduced to $1 \mathrm{slpm}$. The $\mathrm{H}_{2} \mathrm{~S}$ level of $12 \mathrm{ppm}$ was comparable to the original measurement at 1.0 slpm. Thus, after 1.5 hours of operation the sorbent was still effective. At the low gas flow rates and concentrations of $\mathrm{H}_{2} \mathrm{~S}$, breakthrough would not be expected to occur for several days, which was longer than practical to run the test. 
An attempt was made to utilize the Wheeler Equation to determine kinetic information regarding the $\mathrm{ZnO}$ reaction with $\mathrm{H}_{2} \mathrm{~S}$ without having to run a test long enough to observe breakthrough.

The Wheeler Equation, originally developed to predict breakthrough of poisonous vapors in gas masks, is based on a simple first-order model of gas sorption on porous solids. Using this equation, the sorbent rate constant and sorbent utilization can be determined, important parameters in designing adsorption filters. One manifestation of the Wheeler Equation is:

Eq. 23

$$
\ln \left(\frac{\mathrm{C}_{x}}{\mathrm{C}_{\mathrm{o}}}\right)=\frac{-\mathrm{k}_{\mathrm{o}} * \mathrm{~W}_{\mathrm{b}}}{\rho_{\mathrm{b}}} *\left(\frac{1}{\mathrm{Q}}\right)+\frac{\mathrm{t} * \mathrm{C}_{\mathrm{o}} * \mathrm{k}_{\mathrm{o}}}{\rho_{\mathrm{b}} * \mathrm{~W}_{\mathrm{s}}}
$$

where $\mathrm{C}_{\mathrm{o}}$ is the inlet concentration $\mathrm{H}_{2} \mathrm{~S}, \mathrm{C}_{\mathrm{x}}$ is the outlet concentration of $\mathrm{H}_{2} \mathrm{~S}$ at time $\mathrm{t}$, $\mathrm{Q}$ is the gas flow rate, $\rho_{\mathrm{B}}$ is the bulk density of the sorbent, $\mathrm{k}_{\mathrm{o}}$ is the sorption rate, and $\mathrm{W}_{\mathrm{s}}$ is the sorption capacity. Note that this form of the Wheeler Equation allows $\mathrm{k}_{\mathrm{o}}$ and $\mathrm{W}_{\mathrm{s}}$ to be determined from a plot of $\ln \left(\mathrm{C}_{\mathrm{x}} / \mathrm{C}_{\mathrm{o}}\right)$ vs. Q. Figure 59 is an attempt to analyze the sorption data of Table 33 by Eq. 23. Although the resulting plot is linear for the limited data available, it resulted in a negative yintercept, which implies a negative sorption capacity. This unphysical result is attributed to the poor accuracy of the dry chemical analysis tubes used to measure these low concentrations of $\mathrm{H}_{2} \mathrm{~S}$ and the small number of data points that could be collected. Future work will employ a gas chromatograph able to measure sulfur compounds to concentrations of less than $1 \mathrm{ppm}$.

\section{MnO-based sorbent desulfurization}

The MnO-based sorbent was tested at $375^{\circ} \mathrm{C}$ and $0.5 \mathrm{slpm}$. As shown Table 34 and Figure 60, the initial $\mathrm{H}_{2} \mathrm{~S}$ concentration leaving the filter was $10 \mathrm{ppm}$ but begin to increase after only 24 minutes of operation. The cause of this early breakthrough is unknown. Possibly the sorbent became deactivated due to an interfering gas in the syngas stream. Another possibility is that the gas began to channel through the fixed bed.

An attempt to use the Wheeler Equation to analyze this data by plotting $\ln \left(\mathrm{C}_{\mathrm{x}} / \mathrm{C}_{\mathrm{o}}\right)$ vs. $\mathrm{t}$ since 16 data points were available from this time series. However, the data showed little evidence of following the theoretical form of the Wheeler Equation. 
Table 32 Desulfurization with $\mathrm{ZnO}$ on $0.02 \% \mathrm{H}_{2} \mathrm{~S}$ with $\mathrm{N}_{2}$ balance at $450^{\circ} \mathrm{C}$

\begin{tabular}{|c|c|}
\hline Pre-test Baseline & \\
\hline Average $\mathrm{H}_{2} \mathrm{~S}$ & $193 \mathrm{ppm}$ \\
Standard Deviation & $3 \mathrm{ppm}$ \\
Sample Rate & $1 \mathrm{slpm}$ \\
\hline FBR Run \#1 & \\
\hline Average $\mathrm{H}_{2} \mathrm{~S}$ & $0 \mathrm{ppm}$ \\
Standard Deviation & $0 \mathrm{ppm}$ \\
Sample Rate & $0.5 \mathrm{slpm}$ \\
\hline FBR Run \#2 & \\
\hline Average $\mathrm{H}_{2} \mathrm{~S}$ & $2 \mathrm{ppm}$ \\
Standard Deviation & $0 \mathrm{ppm}$ \\
Sample Rate & $1 \mathrm{slpm}$ \\
\hline Post-test Baseline & \\
\hline Average $\mathrm{H}_{2} \mathrm{~S}$ & $195 \mathrm{ppm}$ \\
Standard Deviation & $5 \mathrm{ppm}$ \\
Sample Rate & $1 \mathrm{slpm}$ \\
\hline
\end{tabular}

Table 33 Syngas desulfurization with $\mathrm{ZnO}$ sorbent at $450^{\circ} \mathrm{C}$

\begin{tabular}{|c|c|c|c|}
\hline $\begin{array}{c}\text { Sample } \\
\text { Rate }\end{array}$ & $\begin{array}{l}\text { Relative Time } \\
(\min )\end{array}$ & Test Section & $\begin{array}{c}\mathrm{H} 2 \mathrm{~S} \\
(\mathrm{ppm})\end{array}$ \\
\hline \multirow{3}{*}{1 LPM } & 0 & \multirow{3}{*}{ Pretest baseline } & 300 \\
\hline & 6 & & 290 \\
\hline & 12 & & 290 \\
\hline \multirow{3}{*}{1 LPM } & 46 & \multirow{3}{*}{ FBR Run \#1 } & 12 \\
\hline & 53 & & 13 \\
\hline & 58 & & 13 \\
\hline \multirow{3}{*}{$1.5 \mathrm{LPM}$} & 66 & \multirow{3}{*}{ FBR Run $\# 2$} & 16 \\
\hline & 73 & & 17 \\
\hline & 78 & & 17 \\
\hline \multirow{3}{*}{ 2.25 LPM } & 90 & \multirow{3}{*}{ FBR Run \#3 } & 20 \\
\hline & 95 & & 21 \\
\hline & 98 & & 20 \\
\hline \multirow{3}{*}{$1 \mathrm{LPM}$} & 109 & \multirow{3}{*}{ FBR Run \#4 } & 12 \\
\hline & 111 & & 12 \\
\hline & 115 & & 12 \\
\hline \multirow{3}{*}{1 LPM } & 122 & \multirow{3}{*}{ Post-test baseline } & 280 \\
\hline & 125 & & 280 \\
\hline & 128 & & 280 \\
\hline
\end{tabular}




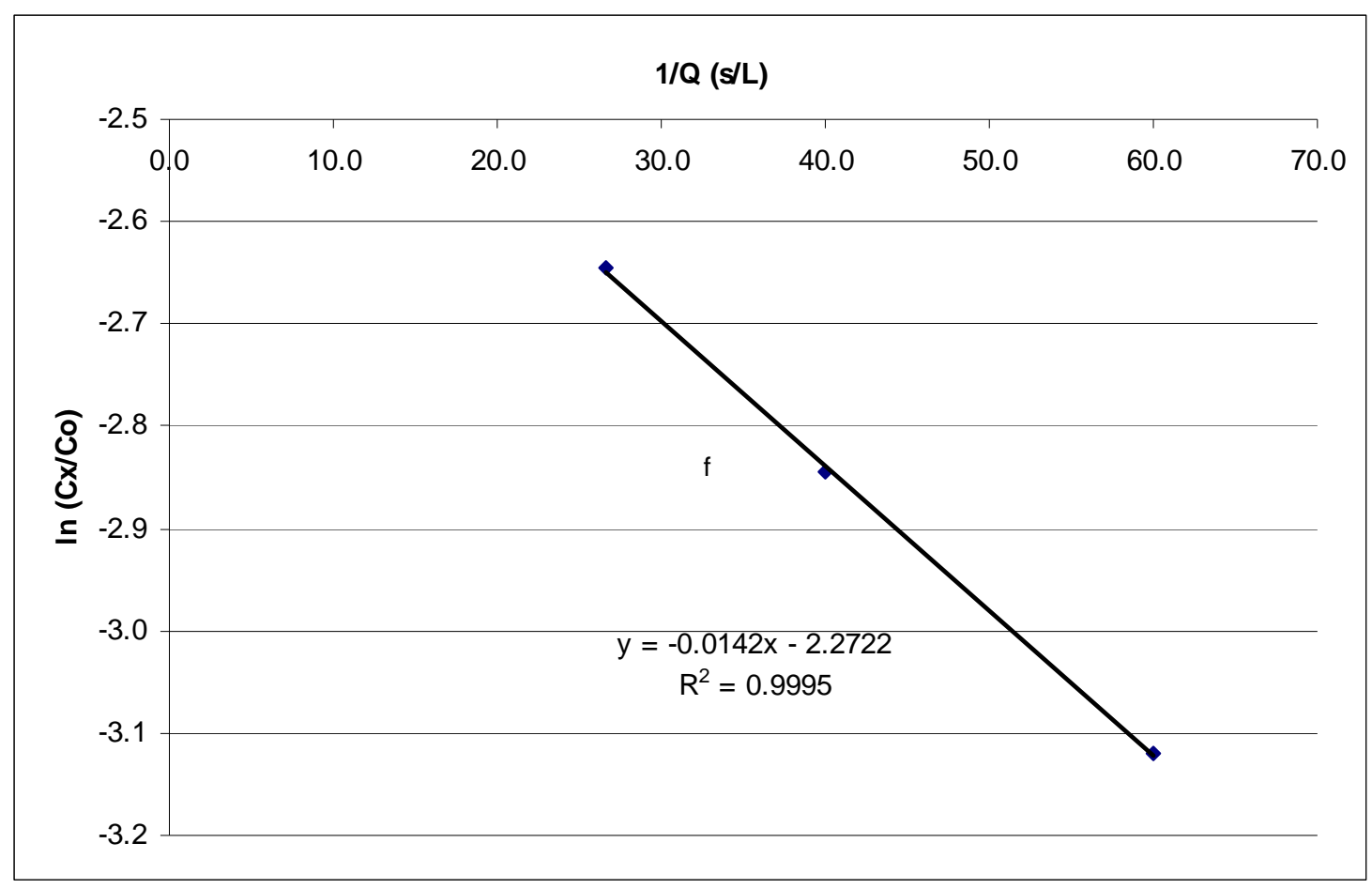

Figure 59 Plot of $\ln \left(\mathrm{C}_{\mathrm{x}} / \mathrm{C}_{\mathrm{x}}\right)$ for FBR08 for use in the Wheeler equation 
Table 34 Syngas desulfurization results with MnO-based sorbent at $375^{\circ} \mathrm{C}$

\begin{tabular}{|c|c|c|}
\hline $\begin{array}{c}\text { Relative Time } \\
\text { (min) }\end{array}$ & $\begin{array}{c}\mathrm{H} 2 \mathrm{~S} \\
\text { (ppm) }\end{array}$ & $\operatorname{In}(\mathrm{Cx} / \mathrm{Co})$ \\
\hline 0 & 32 & -2.15 \\
4 & 22 & -2.53 \\
7 & 17 & -2.78 \\
11 & 13 & -3.05 \\
16 & 10.0 & -3.31 \\
24 & 9 & -3.42 \\
29 & 23 & -2.48 \\
32 & 34 & -2.09 \\
36 & 31 & -2.18 \\
39 & 31 & -2.18 \\
42 & 37 & -2.01 \\
48 & 60 & -1.52 \\
54 & 150 & -0.61 \\
60 & 130 & -0.75 \\
65 & 100 & -1.01 \\
70 & 160 & -0.54 \\
\hline
\end{tabular}

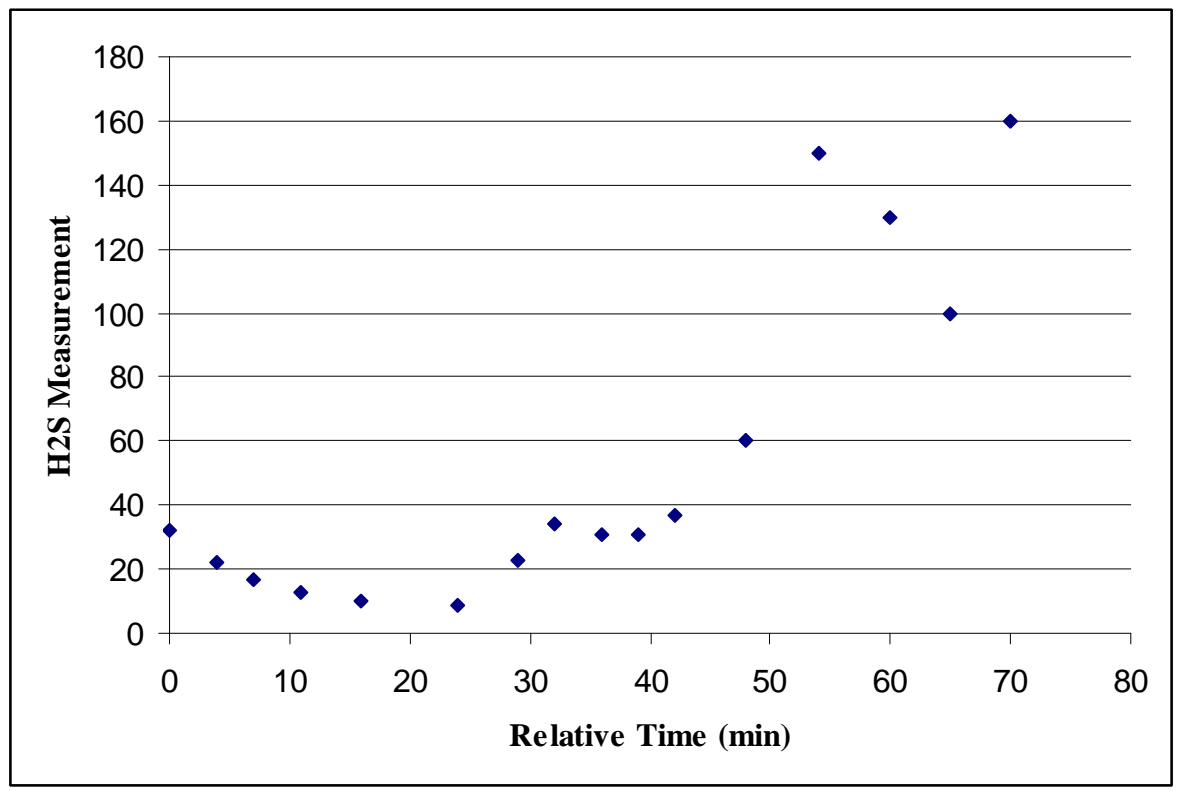

Figure $60 \mathrm{H}_{2} \mathrm{~S}$ measurement vs time using $\mathrm{MnO}$ sorbent at $375^{\circ} \mathrm{C}$ 


\subsection{Analysis of tar measurement methods}

\section{The Effect of Sample Aging on the Accuracy of the IEA Protocol}

Single flask storage was the first storage protocol evaluated. Figure 61 shows the results for single flask storage over six day of a tar/DCM sample obtained from the gasifier operated $736^{\circ} \mathrm{C}$. The apparent tar concentration decreased nearly $35 \%$, from $7.44 \mathrm{~g} / \mathrm{Nm}^{3}$ to $4.86 \mathrm{~g} / \mathrm{Nm}^{3}$ over five days. Since the change occurred so rapidly, a second evaluation was performed over a shorter time interval. Figure 62 shows the results for single flask storage over 15 hours for a tar/DCM sample obtained from the gasifier operated $670^{\circ} \mathrm{C}$. Over this time interval the apparent tar concentration decreases $20 \%$ from $7.29 \mathrm{~g} / \mathrm{Nm}^{3}$ down to $5.70 \mathrm{~g} / \mathrm{Nm}^{3}$.

This apparent loss of tar over the course of time is hypothesized to result from the repeated cooling and warming of the single storage flask between evaluations. Every time the flask was warmed, light tar fractions likely evaporated from the tar/DCM solution and escaped from the flask when it was opened to remove a DCM/tar sample. In an effort to avoid this problem, the multiple flask storage protocol was adopted, which results in a flask being unsealed only once in the course of the experiments.

Multiple flask storage trials were performed over five days for gasification temperatures of $650^{\circ} \mathrm{C}, 732^{\circ} \mathrm{C}$, and $788^{\circ} \mathrm{C}$, respectively. It should be noted that the measurement obtained at zero days corresponds to a sample that was analyzed immediately upon conclusion of the gasification trial and, thus, was not refrigerated. It is assumed to represent the most accurate determination of tar concentration since devolaltilzation and/or polymerization of aged samples are expected to change the characteristics of the sample. The other data points shown in these figures were analyzed at 24 hour intervals after collection of the tar samples.

Tar measurements obtained at the lowest gasification temperature (Figure 63) show a trend toward increasing tar concentration as the sample is aged, although most of these values fall within the uncertainty of the tar measurements, which was taken to be the $95 \%$ confidence interval for four replications. However, at the two higher gasification temperatures (Figure 64 Figure 65) very clearly the indicated tar concentration increases as the age of the sample increases, increasing by $32 \%$ for the gasification trial at $732{ }^{\circ} \mathrm{C}$ and by $55 \%$ for the gasification trial at $788^{\circ} \mathrm{C}$. There is some evidence that the measurements level off after 3-5 days, although this cannot be claimed with certainty. Notice also that tar concentrations decrease with increasing gasification temperature, which is expected for biomass gasification ${ }^{32}$.

A possible reason for this behavior over time is the polymerization of relatively low boiling point compounds into higher boiling point compounds. The evaporation method of the IEA protocol allows lower molecular weight compounds (those with boiling points less than about $105^{\circ} \mathrm{C}$ ) to evaporate leaving behind only "heavy tar" to be determined by the IEA protocol. Thus, aging would convert light tars into heavy tar, which is the only fraction detected by the IEA protocol. The fact that aging effects grow more prominent with increasing gasification temperature suggests that a larger fraction of compounds susceptible to polymerization are created as gasification temperature increases. In general, increasing gasification temperature is expected to 
crack many organic compounds to lower molecular weight, non-condensable compounds, but those condensable compounds that remain show a higher fraction of aromatics ${ }^{32}$.

To explore the effect of polymerization on apparent tar concentration in the earliest stage after sampling, two tests under the same conditions of trials 3 and 5 were conducted with sampling performed in the fifteen hours immediately after completion of the gasification trials. The results are plotted in Figure 66 and Figure 67, respectively. For gasification at $650^{\circ} \mathrm{C}$, the tar concentration increases by $2.5 \%$ from $6.50 \mathrm{~g} / \mathrm{Nm} 3$ to $6.65 \mathrm{~g} / \mathrm{Nm} 3$. The anomalously low concentration in Figure 66 measured after 6 hours of aging cannot be explained. For gasification at $788{ }^{\circ} \mathrm{C}$, the tar concentration increased $10 \%$ from $1.56 \mathrm{~g} / \mathrm{Nm} 3$ to $1.73 \mathrm{~g} / \mathrm{Nm} 3$ (Figure 67). Thus, it would appear that aging occurs very rapidly and errors in tar concentrations can result from storing tar/DCM samples for as little as six hours.

Comparison Between the IEA Protocol and the Hot Condenser Methodology

To validate the accuracy, reproducibility and practicability of tar condenser determination method, tests were conducted to compare the hot tar condenser method to the IEA Protocol (evaporative version). Table 35 details the operating conditions for the fluidized bed gasifier and the sampling system. Although only one equivalence ratio was evaluate, three different temperatures were employed in order to test the methodologies over a broad range of temperatures.

Table 35 Testing conditions for tar comparison

\begin{tabular}{|l|c|}
\hline Gasification Temperature $\left({ }^{\circ} \mathrm{C}\right)$ & $650,705,788$ \\
\hline Equivalence ratio & 0.3 \\
\hline Feedstock & Corn \\
\hline Individual Sampling flowrate (LPM) & 2 \\
\hline Individual Sampling Period (min) & $\sim 70$ \\
\hline
\end{tabular}

To assure a valid comparison between the two methodologies, the two tar collection systems were run in parallel with data collected for one hour from the same producer gas stream. At this point the IEA Protocol system was shut down because of the difficulty in substituting another impinger train for the first. However, the simplicity of the hot condenser allowed a second pressure cooker loaded with fresh tubing and aerosol condenser to be substituted for the first, allowing a second test of this system under comparable gasifier operating conditions. Thus, the first hour of testing allowed the relative accuracy of the two methods to be compared while the second hour of testing provided for limited evaluation of the precision of the hot condenser method of tar evaluation.

The tar analysis for DCM evaporation method at $105^{\circ} \mathrm{C}$ was performed immediately after sampling since it had been found that the age of the tar/DCM sample affects the results of the tar analysis. For each DCM-tar sample, the results were reported as the average of five parallel 


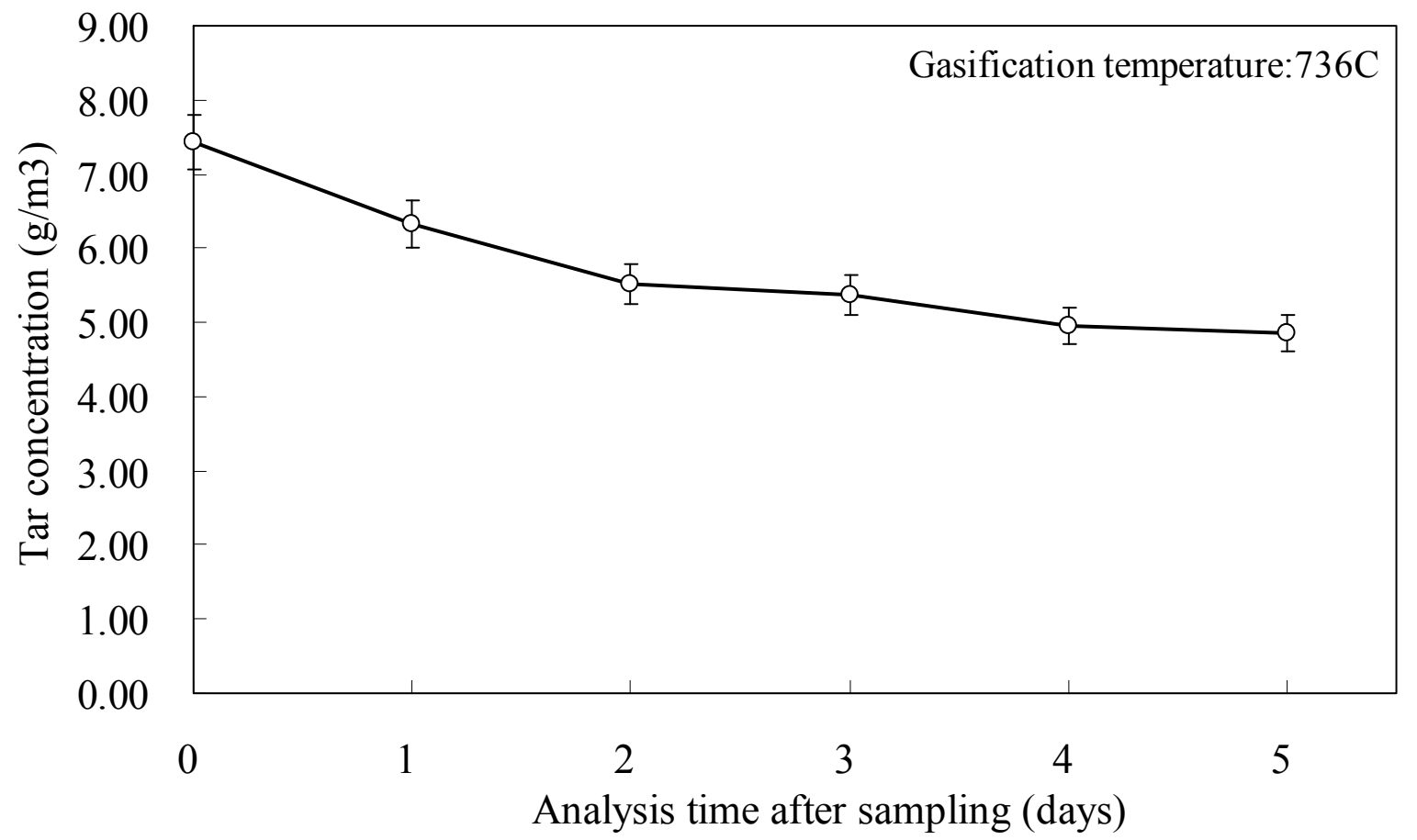

Figure 61 Tar concentration vs. time for single flask storage over six days (gasification at $736^{\circ} \mathrm{C}$ ) 


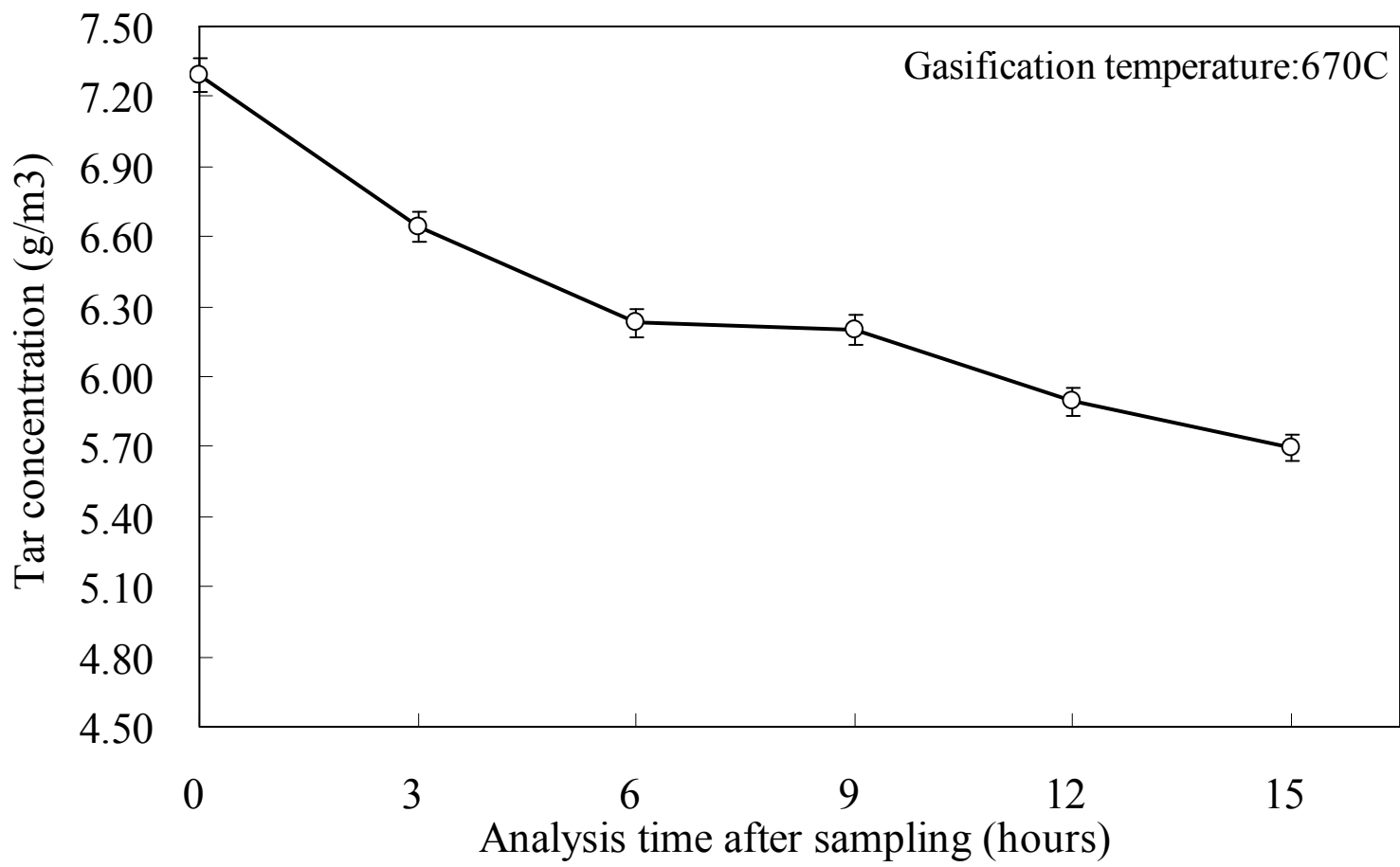

Figure 62 Tar concentration vs. time for single flask storage over 15 hours (gasification at $670^{\circ} \mathrm{C}$ ) 


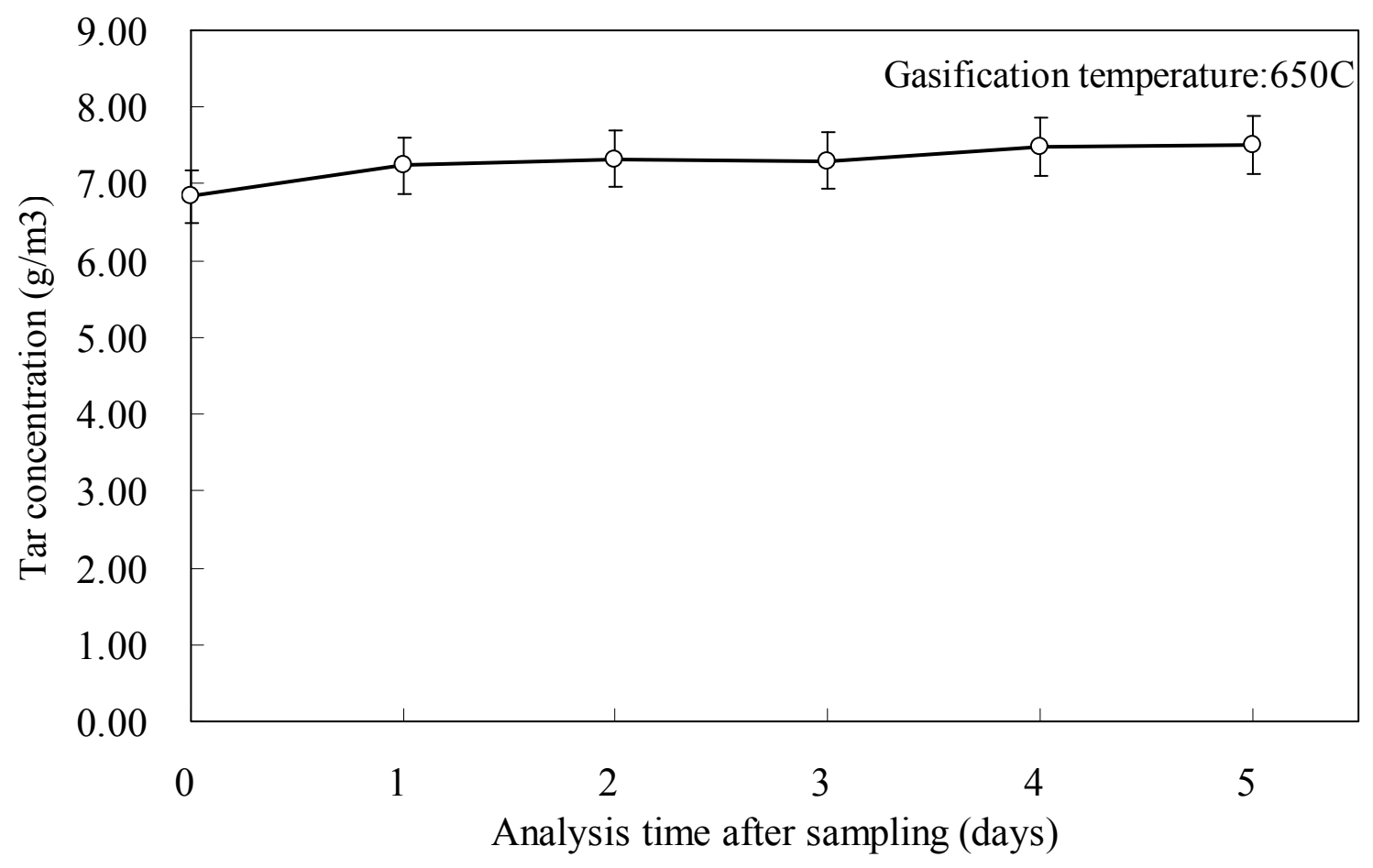

Figure 63 Tar concentration vs. time for multiple flask storage over six days (gasification at $650^{\circ} \mathrm{C}$ ) 


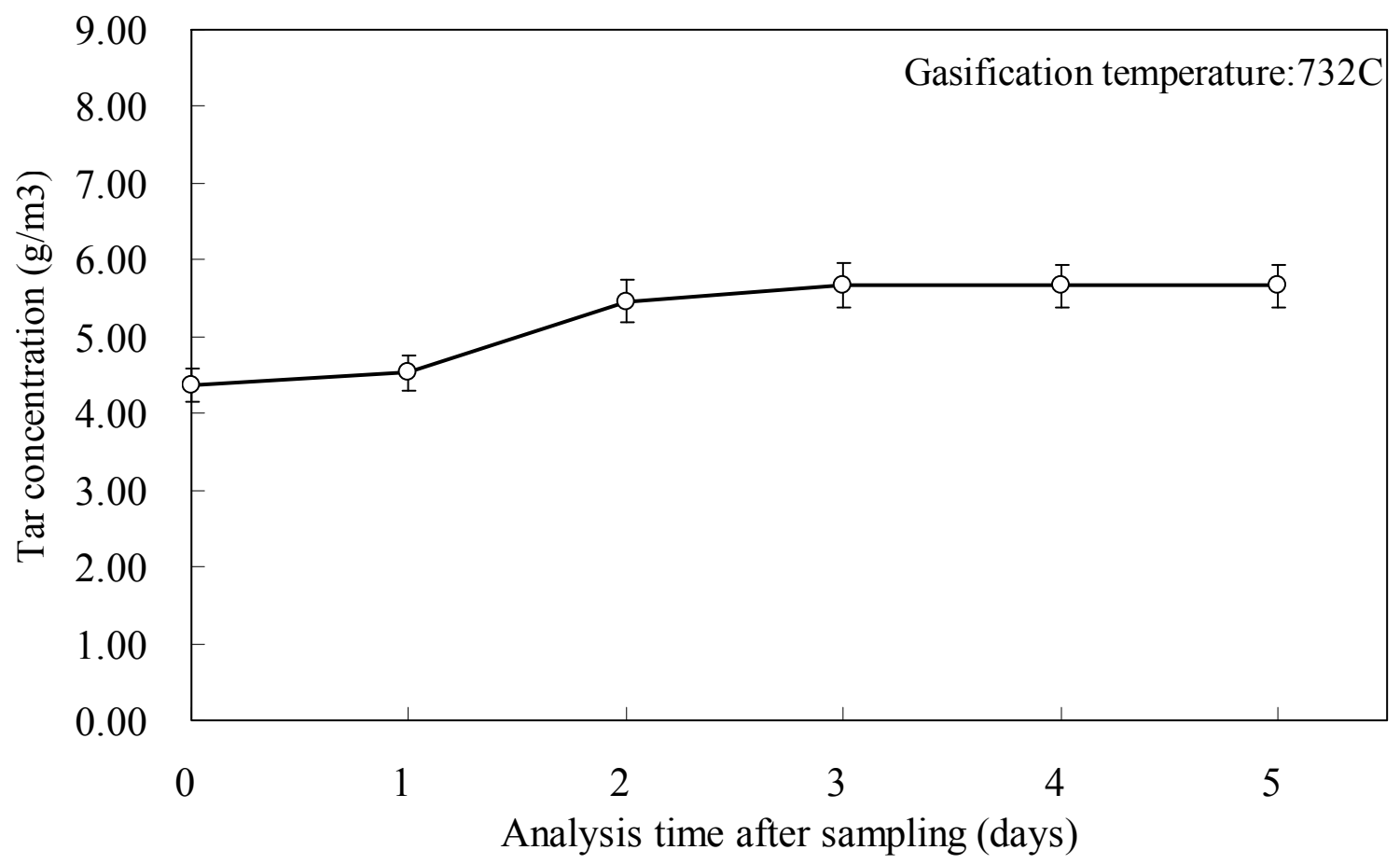

Figure 64 Tar concentration vs. time for multiple flask storage over six days (gasification at $732^{\circ} \mathrm{C}$ ) 


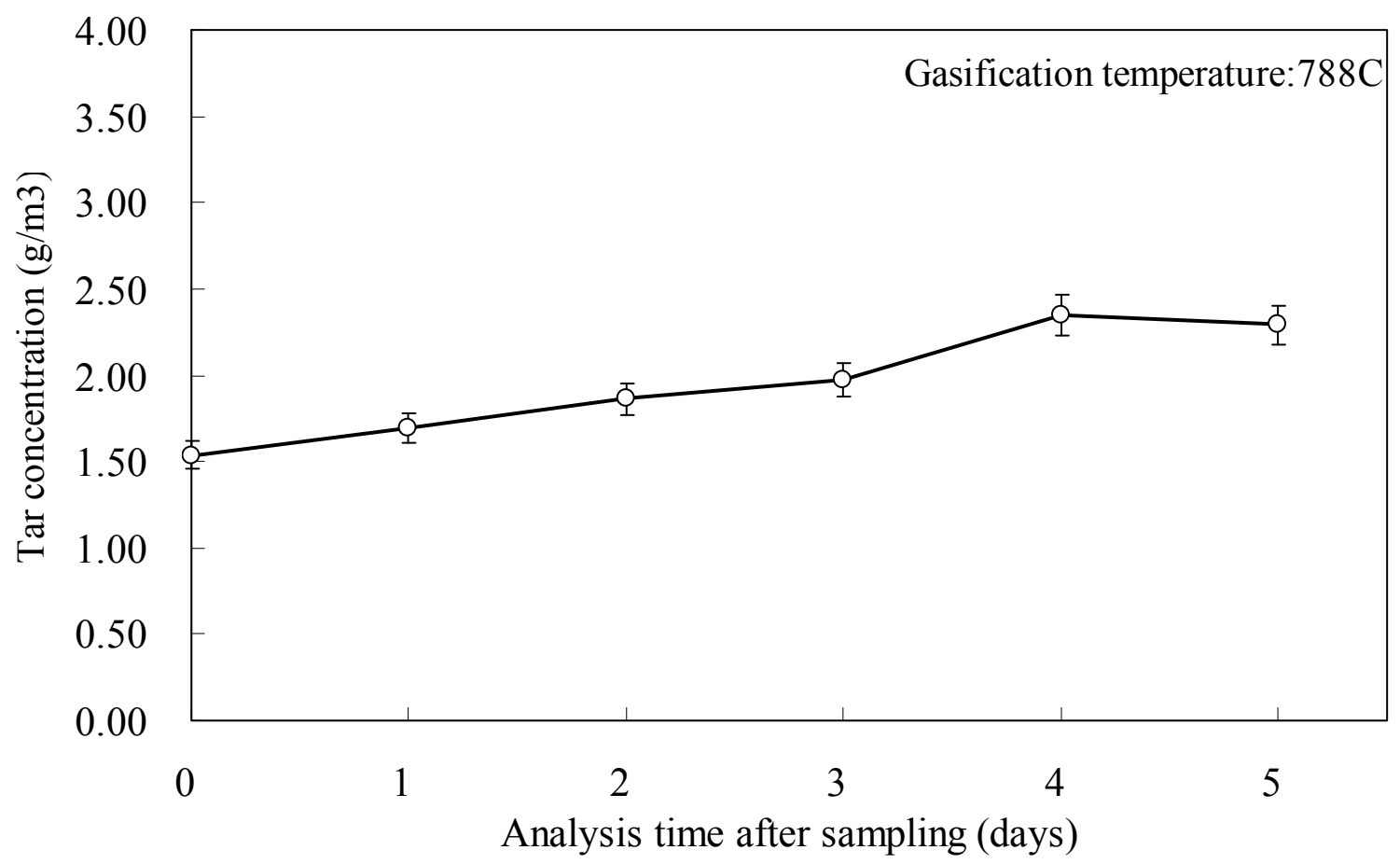

Figure 65 Tar concentration vs. time for multiple flask storage over six days (gasification at $788^{\circ} \mathrm{C}$ ) 


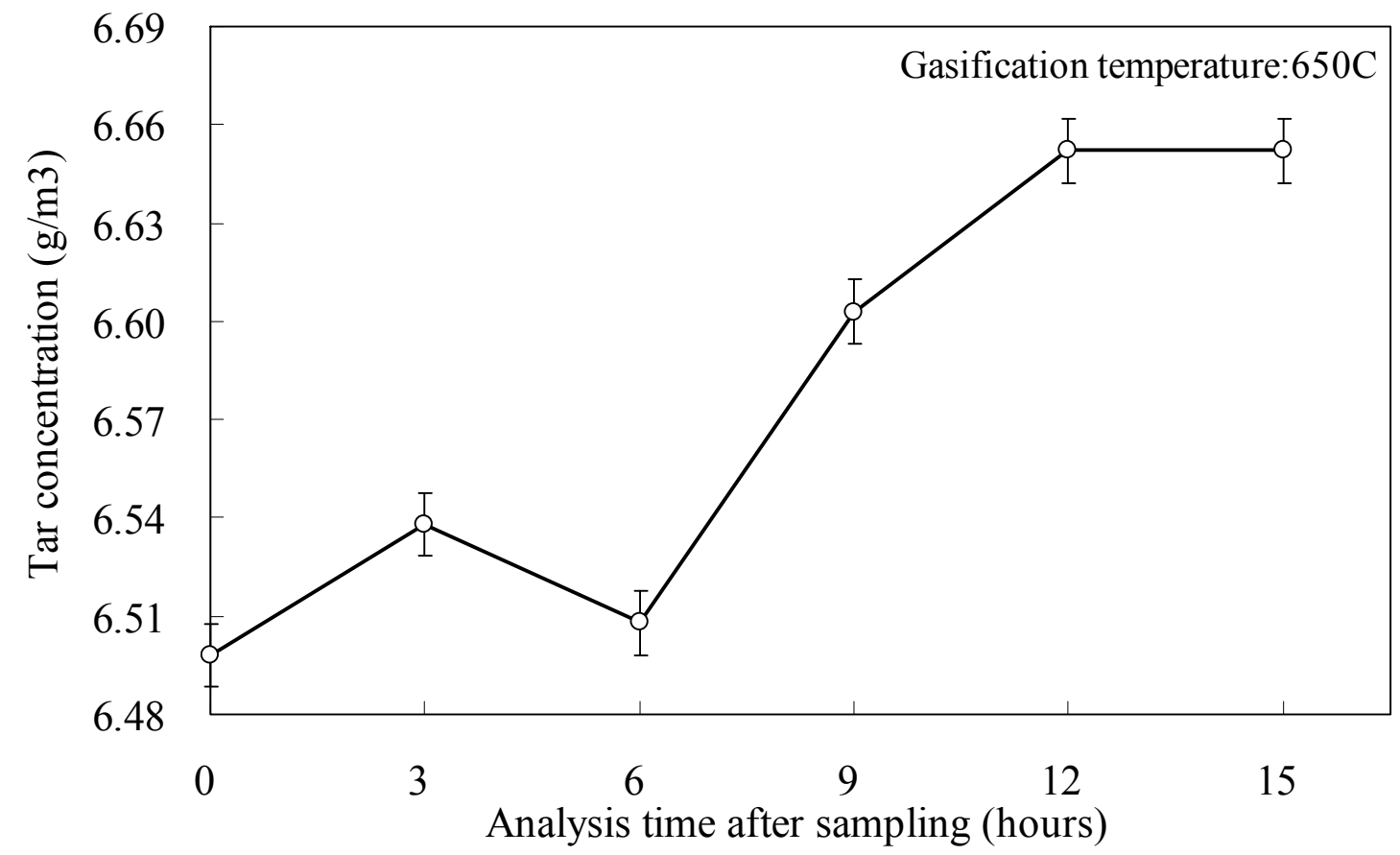

Figure 66 Tar concentration vs. time for multiple flask storage over 15 hours (gasification at $650^{\circ} \mathrm{C}$ ) 


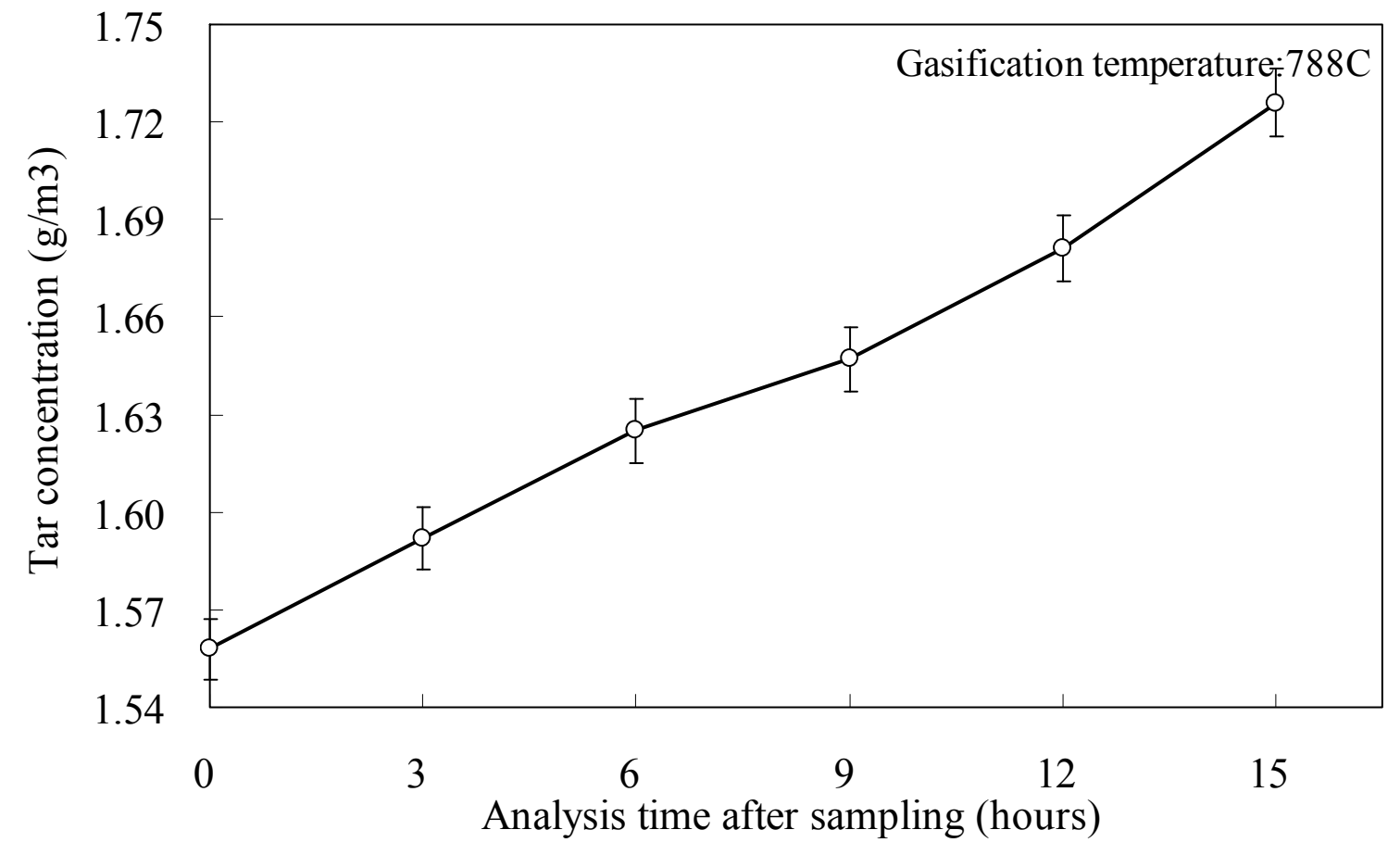

Figure 67 Tar concentration vs. time for multiple flask storage over 15 hours (gasification at $788^{\circ} \mathrm{C}$ ) 
evaporation determinations at $105^{\circ} \mathrm{C}$ and ambient pressure to minimize analysis errors, as described in IEA Protocol. Also, the blank determinations with pure DCM were made to minimize potential errors. Table 36 summarizes the results of the series evaluations of the hot condenser method of tar measurement. From the data it is evident that the repeatability of the tar condenser method is very good, both during a single gasification trial and between gasification trials at the same temperature. Replications during a single gasification trial suggest the method is precise to within 5\%. Variation between gasification trials at the same temperature are less than $10 \%$.

Table 37 summarizes the comparison between the two methodologies. The values for the hot condenser method are the average of the two, one-hour replications shown in Table 36. As previously described, replications during a given gasification trial were not possible for the IEA Protocol. However, comparison of trials at the same temperature (which was an attempt to replicate gasifier operating conditions) gave variations for this method that were less than about $10 \%$. Thus, the precision of the hot condenser appears to be as good if not better than the IEA Protocol.

Determination of the accuracy of the hot condenser method can only be made in comparison with the European protocol, which is considered the standard for tar determinations. Averaged values of replications were used for this determination, as shown in Figure 68. By this measure, the hot condenser method averaged about 5\% uncertainty, with no measurement exceeding $6 \%$ uncertainty. Thus, the hot condenser method appears to be an excellent alternative to the evaporative version of the European protocol.

Because water is also recovered by the IEA Protocol and the hot condenser method, an attempt was made to measure water vapor in the producer gas. Although reasonably good repeatability was achieved for both of these methods, as shown in Figure 69 the IEA Protocol generally measured lower water vapor concentrations than the hot condenser method. The reasons for this are unclear and are the subject of future investigations. Nevertheless, this discrepancy in water vapor measurement does not detract from the conclusions regarding measurement of tar concentrations. 
Table 36 Tar measurements: Serial trials of hot condensers

\begin{tabular}{|c|c|c|c|c|c|}
\hline $\begin{array}{c}\text { Gasification } \\
\text { Trial }\end{array}$ & $\begin{array}{c}\text { Gasification } \\
\text { Temperature } \\
\left({ }^{0} \mathrm{C}\right)\end{array}$ & \multicolumn{2}{|c|}{$\begin{array}{c}\text { Hot Condenser } \\
\text { (first hour }\end{array}$} & \multicolumn{2}{|c|}{$\begin{array}{c}\text { Hot Condenser } \\
\text { (second hour })\end{array}$} \\
\cline { 3 - 6 } & $\begin{array}{c}\text { Tar } \\
\text { Loading } \\
\left(\mathrm{g} / \mathrm{m}^{3}\right)\end{array}$ & $\begin{array}{c}\text { Water } \\
\text { Loading } \\
\left(\mathrm{g} / \mathrm{m}^{3}\right)\end{array}$ & $\begin{array}{c}\text { Tar } \\
\text { Loading } \\
\left(\mathrm{g} / \mathrm{m}^{3}\right)\end{array}$ & $\begin{array}{c}\text { Water } \\
\text { Loading } \\
\left(\mathrm{g} / \mathrm{m}^{3}\right)\end{array}$ \\
\hline 1 & 705 & 8.31 & 265.21 & 7.77 & 217.78 \\
\hline 2 & 705 & 9.80 & 286.81 & 9.60 & 261.77 \\
\hline 3 & 788 & 7.60 & 120.21 & 7.48 & 128.42 \\
\hline 4 & 788 & 8.03 & 137.32 & 7.95 & 153.85 \\
\hline 5 & 650 & 15.63 & 329.27 & 15.12 & 366.93 \\
\hline 6 & 650 & 14.27 & 291.79 & 14.94 & 321.72 \\
\hline
\end{tabular}

Table 37 Tar measurements: Parallel trials of the hot condenser method and the IEA Protocol

\begin{tabular}{|c|c|c|c|c|c|}
\hline \multirow[t]{2}{*}{$\begin{array}{c}\text { Gasification } \\
\text { Trial }\end{array}$} & \multirow[t]{2}{*}{$\begin{array}{l}\text { Gasification } \\
\text { Temperature } \\
\left({ }^{0} \mathrm{C}\right)\end{array}$} & \multicolumn{2}{|c|}{$\begin{array}{c}\text { Hot condensers } \\
\text { (averaged over two, one- } \\
\text { hour trials) }\end{array}$} & \multicolumn{2}{|c|}{ IEA Protocol } \\
\hline & & $\begin{array}{c}\text { Tar } \\
\text { Loading } \\
\left(\mathrm{g} / \mathrm{m}^{3}\right)\end{array}$ & $\begin{array}{l}\text { Water } \\
\text { Loading } \\
\left(\mathrm{g} / \mathrm{m}^{3}\right)\end{array}$ & $\begin{array}{c}\text { Tar } \\
\text { Loading } \\
\left(\mathrm{g} / \mathrm{m}^{3}\right)\end{array}$ & $\begin{array}{l}\text { Water } \\
\text { Loading } \\
\left(\mathrm{g} / \mathrm{m}^{3}\right)\end{array}$ \\
\hline 1 & 705 & 8.04 & 241.5 & 8.90 & 181.06 \\
\hline 2 & 705 & 9.70 & 274.3 & 10.30 & 169.13 \\
\hline 3 & 788 & 7.54 & 124.3 & 8.03 & 126.20 \\
\hline 4 & 788 & 7.99 & 145.6 & 8.44 & 117.59 \\
\hline 5 & 650 & 15.37 & 348.10 & 16.50 & 245.61 \\
\hline 6 & 650 & 14.60 & 306.75 & 14.81 & 190.56 \\
\hline
\end{tabular}




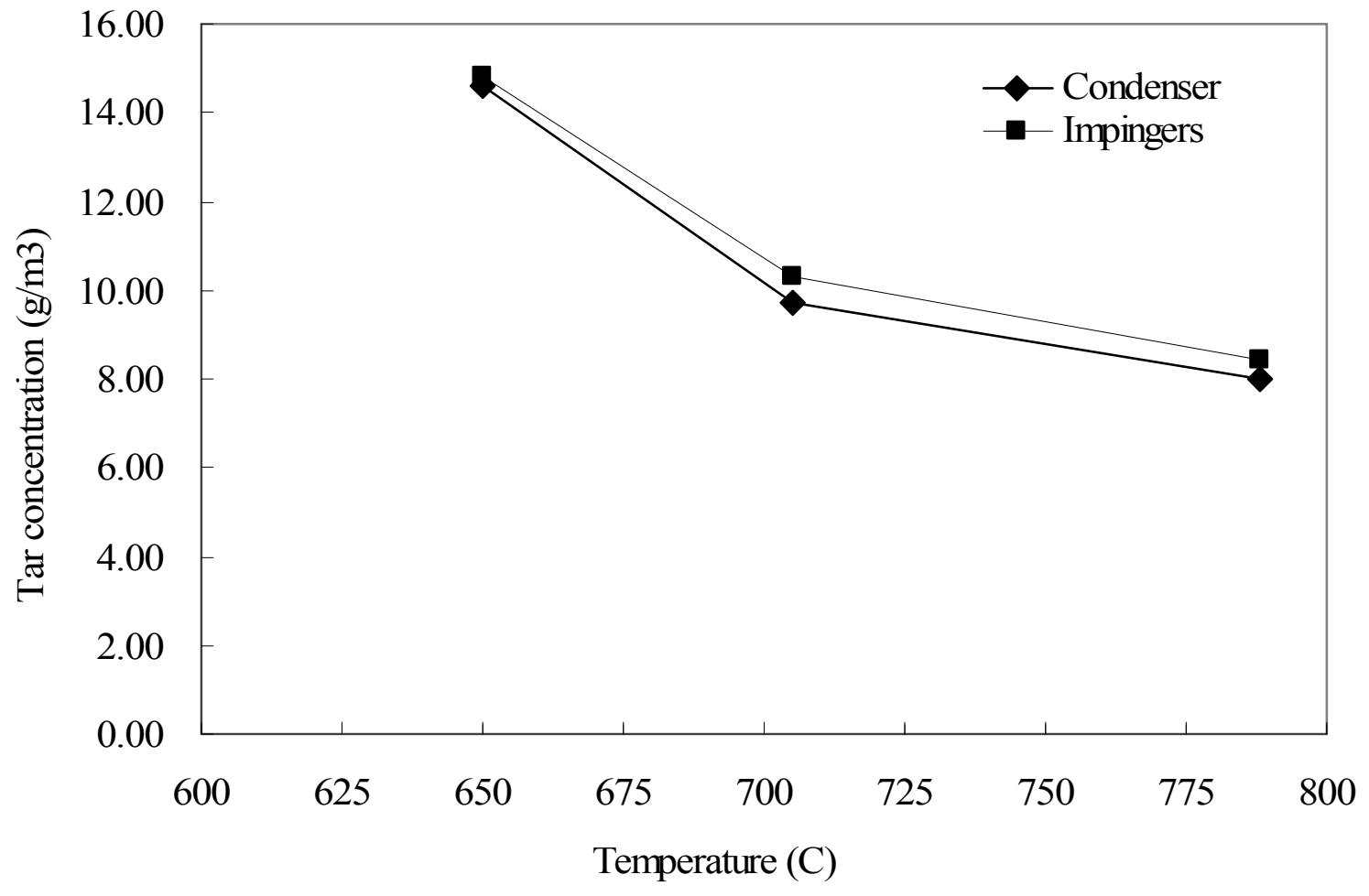

Figure 68 Comparison of tar concentration as determined by the hot condenser method and the IEA Protocol (impingers) 


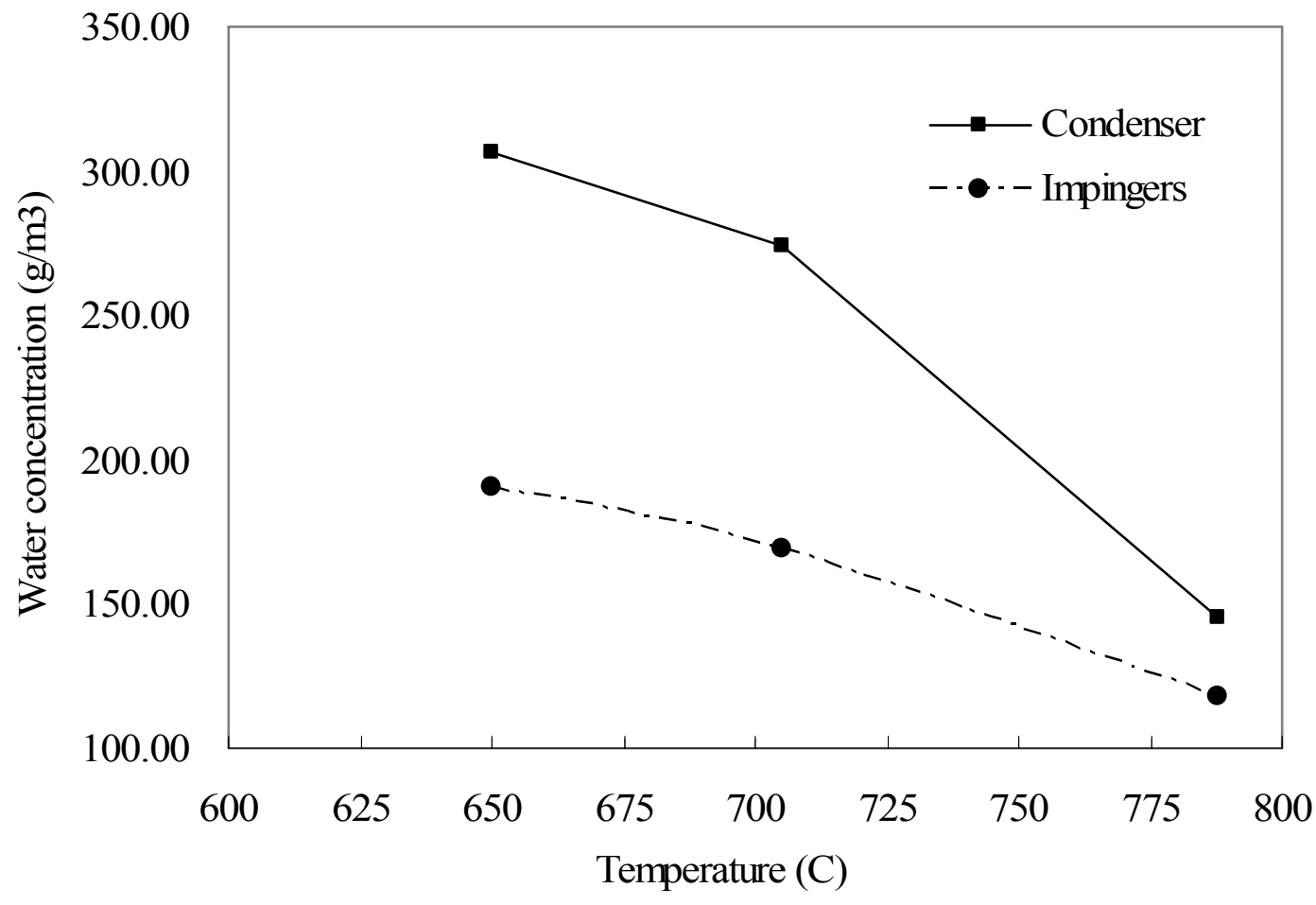

Figure 69 Comparison of water concentration as determined by the hot condenser method and the IEA Protocol (impingers) 


\section{Ballasted Gasifier Model}

A mathematical model of the ballasted gasifier was previously developed that incorporated heat transfer and chemical reaction during the pyrolysis phase of the gasification cycle. However, discrepancies were found between model predictions and experimental data. A complete description of this model is found in Reference 33. An important shortcoming of this model was discrepancies between predicted and actual cooling curves for the ballasted gasifier. In particular, the model predicted a pronounced isothermal event during cooling of the ballasted gasifier that was not evident in the experimental data. The original study hypothesized the existence of a receding liquid phase within the ballast tubes that progressively decreased the rate of heat transfer from the tubes. ${ }^{33}$ The present study attempts to account for this receding liquid phase and more correctly simulate cooling curves for the gasifier. These results are to appear in Biomass and Bioenergy. ${ }^{34}$

\subsection{Model Formulation}

\section{$\underline{\text { Nomenclature }}$}

$\begin{array}{ll}B i & \text { Biot number }(-) \\ c_{p} & \text { specific heat }\left(\mathrm{J} \mathrm{kg}^{-1} \mathrm{~K}^{-1}\right) \\ h & \text { heat transfer coefficient }\left(\mathrm{W} \mathrm{m}^{-2} \mathrm{~K}^{-1}\right) \\ \Delta h_{p y r} & \text { heat of reaction for pyrolysis process }\left(\mathrm{W} \mathrm{kg}^{-1}\right) \\ H_{b e d} & \text { height of the fluidized bed }(\mathrm{m}) \\ k & \text { thermal conductivity }\left(\mathrm{W} \mathrm{m}^{-1} \mathrm{~K}^{-1}\right) \\ l & \text { length }(\mathrm{m}) \\ L_{c} & \text { characteristic dimension }(\mathrm{m}) \\ m & \text { mass }(\mathrm{kg}) \\ \dot{m} & \text { mass flow rate }(\mathrm{kg} \mathrm{s}) \\ n_{t u b e s} & \text { number of ballast tubes within reactor }(-) \\ \dot{Q} & \text { heat transfer rate }(\mathrm{W}) \\ r & \text { radial position from centerline of reactor or tube }(\mathrm{m}) \\ t & \text { time }(\mathrm{s}) \\ T & \text { temperature }(\mathrm{K})\end{array}$

\section{$\underline{\text { Subscripts }}$}

$\begin{array}{ll}\text { ballast } & \text { overall ballast } \\ \text { ext } & \text { external } \\ \text { int } & \text { internal } \\ \mathrm{LiF} & \text { lithium fluoride } \\ \mathrm{ls} & \text { liquid/solid interface } \\ \mathrm{N}_{2} & \text { purge gas (nitrogen) }\end{array}$


SS stainless steel

steam fluidizing steam

tube ballast tube

wall reactor wall

\section{Theory}

The previous formulation of the model treated the lithium fluoride within each ballast tube as an isothermal lumped capacitance during the phase-change period. Thus, the previous model will be referred to as the Lumped Capacitance (LC) model. Lumped capacitance analysis is only appropriate under the following condition ${ }^{35}$ :

Eq. $24 \quad B i=\frac{h \cdot L_{c}}{k}<0.1$

where $B i$ is the Biot number; $L_{c}$ is the characteristic dimension of the thermal mass; $h$ is the convective heat transfer coefficient at the exterior of the mass; and and $k$ is the thermal conductivity of the mass. The characteristic dimension of the cylindrical tubes is half the radius of the tube, or $0.00635 \mathrm{~m}$. Convection coefficients for objects immersed in fluidized beds of relative fine particles are on the order of $300 \mathrm{~W} \mathrm{~m}^{-2} \mathrm{~K}^{-1}$ and the thermal conductivity of lithium fluoride is approximately $4.0 \mathrm{~W} \mathrm{~m}^{-1} \mathrm{~K}^{-1}$, as reported by Pletka ${ }^{36}$. Thus, the value of the Biot number for the ballast system is 0.457 , which is in excess of the maximum value for application of a lumped capacitance analysis (see Table 38).

Table 38 Calculation of the Biot number, $B i$, for ballast system

\begin{tabular}{rccc}
\hline$L_{c}(\mathrm{~m})$ & $h\left(\mathrm{~W} \mathrm{~m}^{-2} \mathrm{~K}^{-1}\right)$ & $k\left(\mathrm{~W} \mathrm{~m}^{-1} \mathrm{~K}^{-1}\right)$ & $B i$ \\
\hline 0.00635 & 300 & 4.0 & 0.476 \\
\hline
\end{tabular}

Furthermore, heat transfer from the phase-change material to the fluidized bed is inhibited by the formation of a solid layer of lithium fluoride on the inside wall of the tube that grows toward the center of the tube as the ballast tubes cool. To effectively characterize this phenomenon, a Receding Interface (RI) model was formulated.

The RI model is illustrated in Figure 70. The phase change material is contained within a stainless steel tube of external radius, $r_{\text {ext }}$, and internal radius, $r_{\text {int }}$. As the lithium fluoride changes phase from liquid (represented by the gray core) to solid (represented by the crosshatched outer layer), a liquid/solid interface is postulated to exist at radius $r_{l s}$, which recedes toward the center of the tube. At the onset of pyrolysis, as illustrated in Figure 70 (a), the RI model assumes that the ballast tubes are filled with molten lithium fluoride; thus:

Eq. 25

$$
r_{l s}=r_{\text {int }} \text {. }
$$


As the lithium fluoride cools to the phase-change temperature and solidification commences, as shown in Figure 70 (b) and (c), the latent heat of fusion is released to the fluidized bed and the liquid/solid interface moves inward; thus:

Eq. $26 \quad r_{l s}<r_{\text {int }}$.

This recession continues until the interface reaches the centerline of the tube, as illustrated in Figure $70(\mathrm{~d})$, when solidification is complete; that is:

Eq. $27 \quad r_{l s} \rightarrow 0$.

Following completion of phase change, the lithium fluoride continues to cool, once again providing sensible heat to the fluidized bed.

\section{Model Assumptions}

The RI model is based on a few important assumptions. These assumptions, established during the work of Oatley ${ }^{37}$ and Pletka ${ }^{36}$, are as follows:

- Heat transfer is one-dimensional and occurs only in the radial direction.

- The bed-to-wall heat transfer coefficient, $h_{\text {wall }}$, and the bed-to-tube heat transfer coefficient, $h_{\text {tube }}$, are assumed to have the same value.

- The bed is uniform in temperature, and the fluidizing gas entering the bed is instantly raised to the temperature of the bed.

- The phase-change material melts at a constant temperature, and completely releases its latent heat of fusion.

The original LC model altered the height of the bed, $H_{b e d}$, to account for axial conduction in the reactor wall. The value of this variable was set higher than its actual value to mimic the effect of axial conduction. In the RI model, this variable was set to its actual value (axial conduction can only be properly accounted for with a two-dimensional representation).

In order to establish the initial conditions within the reactor, the following additional assumptions were established:

- The inner refractory wall temperature is $30 \mathrm{~K}$ less than the average bed temperature. This assumption is based on observations from previous research ${ }^{36}$.

- The temperatures of the inner and outer surfaces of the steel reactor shell are equal due to the relatively high thermal conductivity of steel.

- The temperature gradients in solid materials are linear.

- The temperature of the ballast is initially equal to that of the bed. 


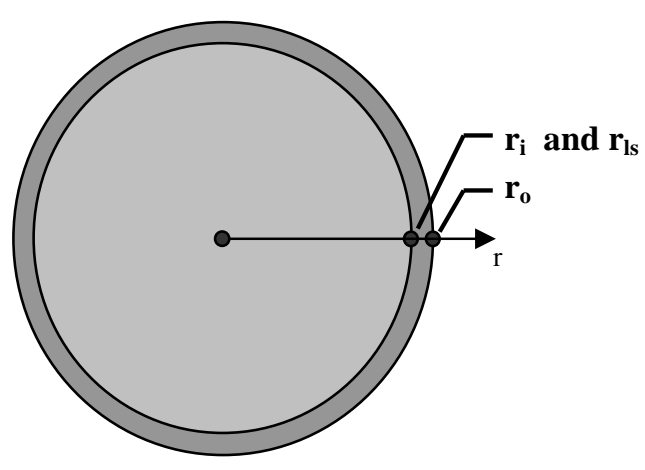

(a)

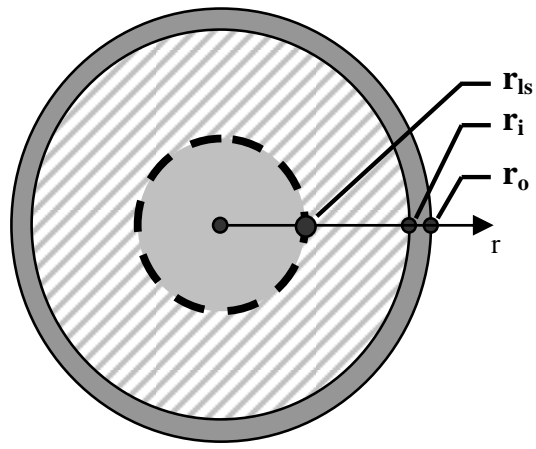

(c)

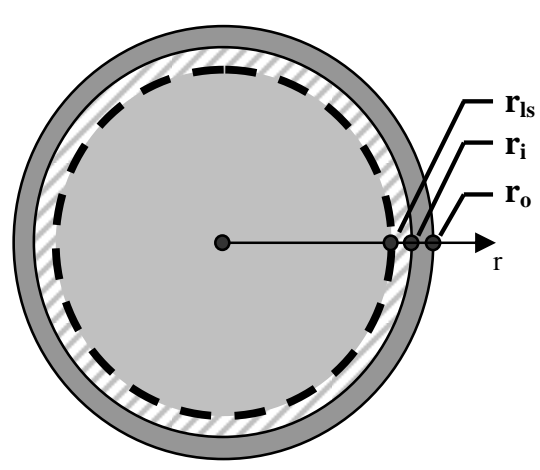

(b)

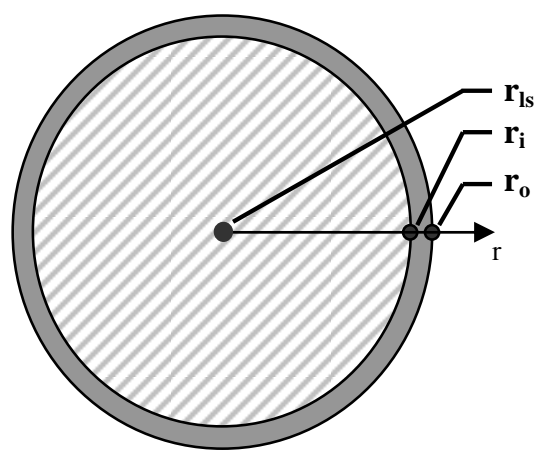

(d)

Figure 70 Illustration of Receding Interface model applied to ballast tubes 


\section{Mathematical Formulation}

The mathematical model was composed of a 12-point nodal network, as illustrated in Figure 71, with additional nodes representing the ballast, steam and nitrogen flow into the reactor and the ambient environment. The model considered a cylindrical reactor with internal refractory, a steel shell and external blanket insulation. By completing an energy balance around each node, a set of differential equations was developed to describe the rate of change of the temperature of each node.

In the case of unballasted gasification, only 12 equations are required to model the pyrolysis process. For node 1, representing the fluidized bed, the temperature is affected by the gases that pass through the reactor, the temperature of the reactor wall, and the chemical energy consumed by the pyrolysis process. Thus the equation for node 1 is as follows:

Eq. 28

$$
\frac{d T_{1}}{d t}=\frac{1}{m_{1} \cdot c_{p, 1}}\left[\dot{Q}_{\text {steam }}+\dot{Q}_{N_{2}}+\dot{Q}_{\text {wall }}+\dot{Q}_{p y r}\right]
$$

where

Eq. 29

$$
\dot{Q}_{\text {steam }}=\dot{m}_{\text {steam }} \cdot c_{p, \text { steam }} \cdot\left(T_{\text {steam }}-T_{1}\right)
$$

Eq. 30

$$
\dot{Q}_{N_{2}}=\dot{m}_{N_{2}} \cdot c_{p, N_{2}} \cdot\left(T_{N_{2}}-T_{1}\right)
$$

Eq. 31

$$
\dot{Q}_{\text {wall }}=2 \cdot \pi \cdot r_{2} \cdot H_{\text {bed }} \cdot h_{\text {wall }} \cdot\left(T_{2}-T_{1}\right)
$$

Eq. 32

$$
\dot{Q}_{p y r}=\dot{m}_{\text {biomass }} \cdot \Delta h_{p y r} \text {. }
$$

For node 2, representing the bed/refractory wall interface, the temperature of the node is affected by convection from the fluidized bed and conduction through the wall:

Eq. 33

$$
\frac{d T_{2}}{d t}=\frac{1}{m_{2} \cdot c_{p, 2}} \cdot\left[2 \cdot \pi \cdot r_{2} \cdot H_{\text {bed }} \cdot h_{\text {wall }} \cdot\left(T_{1}-T_{2}\right)+\frac{2 \cdot \pi \cdot k_{2} \cdot l}{\ln \left(r_{3} / r_{2}\right)} \cdot\left(T_{3}-T_{2}\right)\right] .
$$

For node 3 through node 11, the nodes not exposed to convection and limited to heat transfer via conduction, the equations are of the form:

Eq. 34

$$
\frac{d T_{i}}{d t}=\frac{1}{m_{i} \cdot c_{p, i}} \cdot\left[\frac{2 \cdot \pi \cdot k_{i-1} \cdot l}{\ln \left(r_{i} / r_{i-1}\right)} \cdot\left(T_{i-1}-T_{i}\right)+\frac{2 \cdot \pi \cdot k_{i} \cdot l}{\ln \left(r_{i+1} / r_{i}\right)} \cdot\left(T_{i+1}-T_{i}\right)\right] .
$$




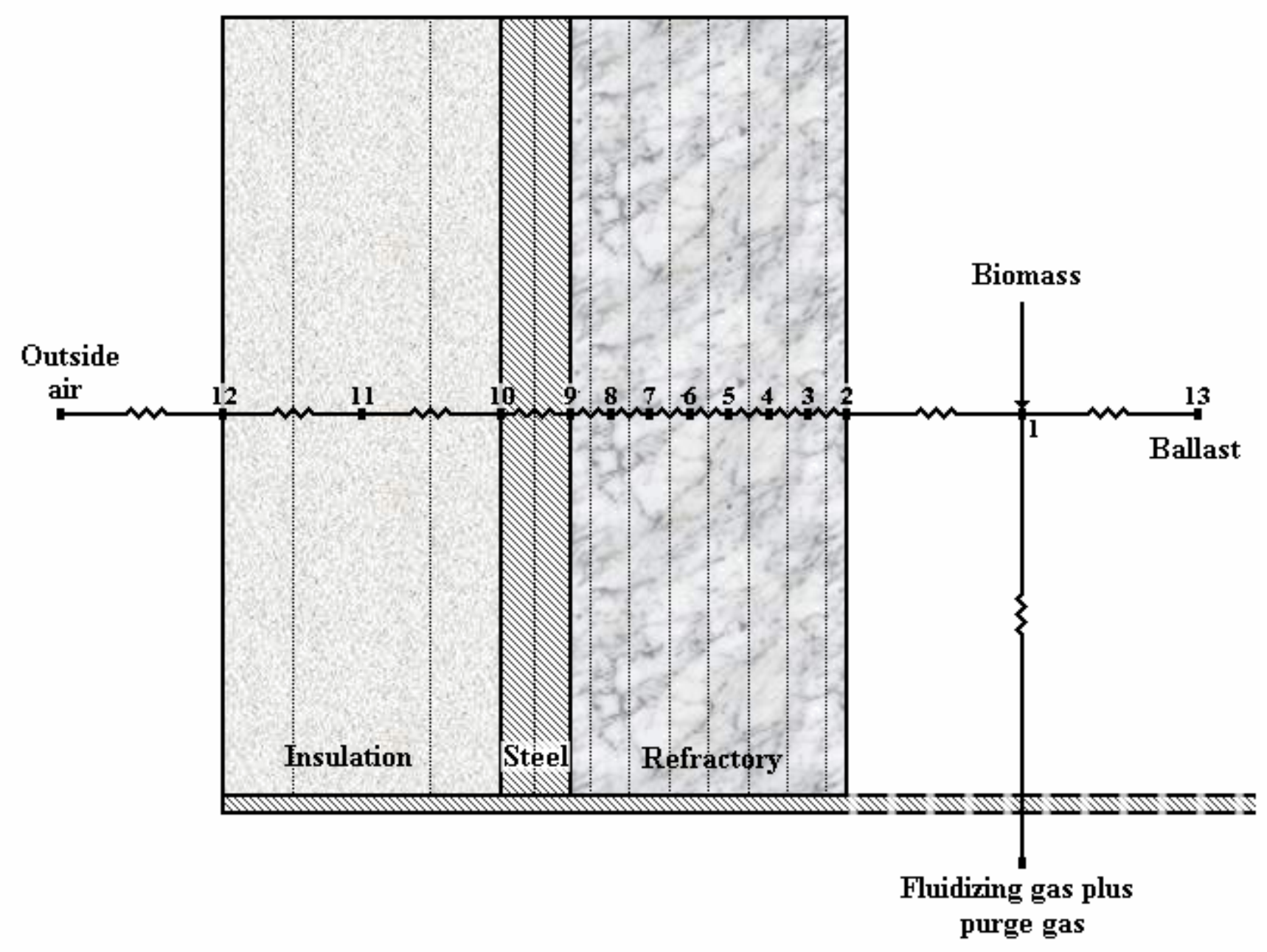

Figure 71 Ballasted gasification nodal network 
Finally, for node 12, representing the blanket insulation/environment interface, the temperature is affected by the conduction through the insulation and interaction with the ambient environment:

Eq. 35

$$
\frac{d T_{12}}{d t}=\frac{1}{m_{12} \cdot c_{p, 12}} \cdot\left[\frac{2 \cdot \pi \cdot k_{11} \cdot l}{\ln \left(r_{12} / r_{11}\right)} \cdot\left(T_{11}-T_{12}\right)+2 \cdot \pi \cdot r_{12} \cdot l \cdot h_{e x t} \cdot\left(T_{a m b}-T_{12}\right)\right] .
$$

It should be noted that the equations developed to describe the unballasted process are identical in both the LC and RI model, as the differences between the models lie in the equations describing the behavior of the ballast tubes.

In the case of ballasted gasification, Eq. 28 must be modified to consider the effect of the ballast upon the fluidized bed, and three sets of equations must be established to describe the behavior of the tubes prior to the phase-change process, during the phase-change process, and following the phase-change process. The equation describing fluidized bed (node 1) becomes:

Eq. 36

$$
\frac{d T_{1}}{d t}=\frac{1}{m_{1} \cdot c_{p, 1}} \cdot\left[\dot{Q}_{\text {steam }}+\dot{Q}_{N_{2}}+\dot{Q}_{\text {wall }}+\dot{Q}_{\text {ballast }}+\dot{Q}_{p y r}\right]
$$

where

Eq. 37

$$
\dot{Q}_{\text {ballast }}=n_{\text {tubes }} \cdot\left[2 \cdot \pi \cdot r_{13} \cdot l_{\text {tube }} \cdot h_{\text {tube }} \cdot\left(T_{13}-T_{1}\right)\right] \text {. }
$$

The ballast is composed of an array of stainless tubes filled with lithium fluoride, a high temperature phase-change material. Prior to the commencement of solidification, temperature gradients within the stainless steel and lithium fluoride are assumed to be negligible. The steel tubes are represented by node 13 , while node 14 represents the phase-change material within the tubes. The equations for the ballast nodes are as follows:

Eq. 38

$$
\frac{d T_{13}}{d t}=\frac{1}{m_{13} \cdot c_{p, 13}} \cdot\left[2 \cdot \pi \cdot r_{\text {ext }} \cdot l_{\text {tube }} \cdot h_{\text {tube }} \cdot\left(T_{1}-T_{13}\right)+\frac{2 \cdot \pi \cdot k_{\text {SS }} \cdot l_{\text {tube,eff }}}{\ln \left(r_{\text {ext }} / r_{\text {int }}\right)} \cdot\left(T_{14}-T_{13}\right)\right]
$$

Eq. 39

$$
\frac{d T_{14}}{d t}=\frac{1}{m_{14} \cdot c_{p, 14}} \cdot\left[\frac{2 \cdot \pi \cdot k_{S S} \cdot l_{\text {tubee eff }}}{\ln \left(r_{\text {ext }} / r_{\text {int }}\right)} \cdot\left(T_{13}-T_{14}\right)\right] .
$$

During phase change, the average temperatures within the stainless steel and lithium fluoride are immaterial to the analysis. Instead, the important temperatures are the temperature of the outer tube surface and the temperature of the liquid/solid interface, which is the source of the released latent heat. The outer tube surface temperature (located at $r_{\text {ext }}$ ) is assumed to be the temperature of Node 13 at the beginning of the phase change period (this is valid due to the high thermal conductivity of stainless steel). The temperature of the liquid/solid interface is specified by the phase change temperature, $T_{p c}=1123 \mathrm{~K}\left(1560^{\circ} \mathrm{F}\right)$, which is constant. Therefore, Node 13 is now located at the outer stainless steel surface, and Node 14 is located at the liquid/solid interface. The heat transfer equations become: 
Eq. 40

$$
\left.\frac{d T_{13}}{d t}=\frac{1}{m_{13} \cdot c_{p, 13}} \cdot\left\{2 \cdot \pi \cdot r_{\text {ext }} \cdot l_{\text {tube }} \cdot h_{\text {tube }} \cdot\left(T_{1}-T_{13}\right)+\frac{2 \cdot \pi \cdot l_{\text {tube,eff }} \cdot\left(T_{14}-T_{13}\right)}{\left[\frac{\ln \left(r_{\text {ext }} / r_{\text {int }}\right)}{k_{\text {SS }}}+\frac{\ln \left(r_{\text {int }} / r_{\text {ls }}\right)}{k_{\text {LiF }}}\right]}\right]\right\}
$$

Eq. $41 \quad \frac{d T_{14}}{d t}=0$

where

$r_{l s} \quad=$ radial position of liquid/solid interface (Node 14) within ballast tube, $\mathrm{m}$

$k_{L i F}=$ thermal conductivity of lithium fluoride, $\mathrm{W} \mathrm{m}^{-1} \mathrm{~K}^{-1}$.

The rate of latent heat transfer to the stainless steel tube is calculated during each time step from an energy balance on the tube:

Eq. 42

$$
\dot{Q}_{\text {latent }}=\frac{2 \cdot \pi \cdot l_{\text {tube,eff }} \cdot\left(T_{14}-T_{13}\right)}{\left[\frac{\ln \left(r_{\text {ext }} / r_{\text {int }}\right)}{k_{S S}}+\frac{\ln \left(r_{\text {int }} / r_{\text {ls }}\right)}{k_{\text {LiF }}}\right]} .
$$

This quantity is important in determining the total latent heat of fusion transferred from the tubes.

The mass of lithium fluoride that solidifies during a time step can be estimated by:

Eq. 43

$$
m_{\text {fusion }}=\frac{\dot{Q}_{\text {latent }} \cdot t_{\text {step }}}{h_{\text {fus }}}
$$

where

$$
\begin{aligned}
& \dot{Q}_{\text {latent }}=\text { latent heat transfer rate, } \mathrm{W} \\
& m_{\text {fusion }}=\text { mass of lithium fluoride solidified during time interval, } \mathrm{kg} \\
& h_{\text {fus }}=\text { latent heat of fusion of lithium fluoride, } \mathrm{J} \mathrm{kg}^{-1} \\
& t_{\text {step }}=\text { time interval of numerical integration method. }
\end{aligned}
$$

The volume of phase change material that has fused in this time interval is:

Eq. 44

$$
V_{\text {fusion }}=\frac{m_{\text {fusion }}}{\rho_{\text {LiF }, s}} .
$$


The change in the position of the liquid/solid interface can then be calculated using the formula for the volume of an annulus:

Eq. $45 \quad V_{\text {fusion }}=\pi \cdot\left(r_{l s, t+1}^{2}-r_{l s, t}^{2}\right) \cdot l_{\text {tube, eff }}$

where

$$
\begin{array}{ll}
V_{f u s i o n} & =\text { volume of lithium fluoride solidified during time interval, } \mathrm{m}^{3} \\
\rho_{L i F, s} & =\text { density of solid lithium fluoride, } \mathrm{kg} \mathrm{m}^{-3} \\
r_{l s, t} & =\text { initial radial position of liquid/solid interface during time interval, } \mathrm{m} \\
r_{l s, t+1} & =\text { final radial position of liquid/solid interface during time interval, } \mathrm{m} .
\end{array}
$$

Solving for radius of the moving interface:

Eq. 46

$$
r_{l s, t+1}=\sqrt{r_{l s, t}^{2}-\frac{V_{\text {fusion }}}{\pi \cdot l_{\text {tube eff }}}} .
$$

The phase change process continues until $r_{l s, t+1}$ reaches zero. At this point, sensible heat transfer resumes until the end of the cooling period.

To accurately model the sensible heat transfer for the remaining cooling period a small modification to Eq. 40 is required. This modification is necessary because a singularity develops as $r_{l s}$ approaches zero (i.e. the natural $\log$ term, $\ln \left(r_{\text {int }} / r_{l s}\right)$, becomes undefined). To counteract this singularity, the variable $r_{l s}$ is incremented to $r_{l s}+0.00001$, as shown in the following equations:

Eq. 47

$$
\begin{aligned}
& \frac{d T_{13}}{d t}=\frac{1}{m_{13} \cdot c_{p, 13}} \cdot\left\{2 \cdot \pi \cdot r_{\text {ext }} \cdot l_{\text {tube }} \cdot h_{\text {tube }} \cdot\left(T_{1}-T_{13}\right)+\frac{2 \cdot \pi \cdot l_{\text {tubeeff }} \cdot\left(T_{14}-T_{13}\right)}{\left[\frac{\ln \left(r_{\text {ext }} / r_{\text {int }}\right)}{k_{\text {SS }}}+\frac{\ln \left(r_{\text {int }} /\left(r_{\text {ls }}+0.00001\right)\right)}{k_{\text {LiF }}}\right]}\right\} \\
& \text { Eq. } 48 \quad \frac{d T_{14}}{d t}=\frac{1}{m_{14} \cdot c_{p, 14}} \cdot\left\{\frac{2 \cdot \pi \cdot l_{\text {tubeeff }} \cdot\left(T_{13}-T_{14}\right)}{\left[\frac{\ln \left(r_{\text {ext }} / r_{\text {int }}\right)}{k_{S S}}+\frac{\ln \left(r_{\text {int }} /\left(r_{\text {ls }}+0.00001\right)\right)}{k_{\text {LiF }}}\right]}\right\}
\end{aligned}
$$


This small alteration has little impact on the behavior of the model and allows for a smooth transition in ballast tube temperature profiles from the period of phase change to the post-phase change period.

As with the previous model, the equation set was solved using a non-stiff differential equation solver. This program employs a fourth-order Runge-Kutta method to integrate the equations and is written in the $\mathrm{C}++$ programming language.

\subsection{Computational Results}

In order to verify the accuracy of the Receding Interface (RI) model and demonstrate that the RI model offered an improvement over the Lumped Capacitance (LC) model, the RI model was used to predict bed temperature profiles during tests in which steam was injected into a hot reactor, but biomass was not fed to the reactor, which simplified the thermal analysis. The profiles predicted by the RI model were compared to both predictions from the LC model and to experimental data from previous research ${ }^{36}$. The temperature profiles for cooling tests with and without phase change ballast in the reactor are shown in Figure 72 and Figure 73, respectively.

The differences between the LC and RI models in the unballasted case are attributed to the slight differences in the formulation of radiation heat transfer in the two models. In the ballasted case, the RI model predicts a monotonic decline in temperature during the phase-change period while the LC model predicts a period of isothermal cooling as the phase-change material solidifies. The continuous temperature decline of the LC model is consistent with experimentally observed behavior of the reactor. However, it is interesting to note that, upon completion of solidification, the cooling curves of the LC and RI models are very similar, both providing relatively accurate cooling curves during the final 45 minutes of the experimental data.

Following the validation of the RI model, sensitivity analyses were performed to identify key parameters influencing performance of the ballasted gasifier. Three parameters of the ballast were investigated: the mass of lithium fluoride per tube, $m_{L i F}$; thermal conductivity of the lithium fluoride, $k_{L i F}$; and the characteristic dimension of the ballast tubes, $L_{c}$.

Sensitivity Analysis 1: Mass of phase-change material per tube

Ideally, a ballasted gasifier would operate isothermally for the duration of the pyrolysis phase of the gasification cycle. The isothermal period is dependent upon the mass of phase-change material contained within the bed, $m_{p c}$, the latent heat of fusion of the phase-change material, $h_{p c, f u s}$, and the rate at which energy is withdrawn from the bed. In the case where the bed is cooled by steam, this rate is equal to the rate of heat transfer from the bed to the steam described by Eq. 29. Isothermal operation of the fluidized bed is never actually achieved because a significant thermal barrier exists between the ballast tubes and the fluidized bed. However, the phase-change material is able to achieve a period of cooling when the rate of temperature drop in the bed is substantially reduced. This period, referred to as the phase-change time, $t_{p c}$, is bounded by two inflection points in the cooling curve. This time can be estimated from the expression: 


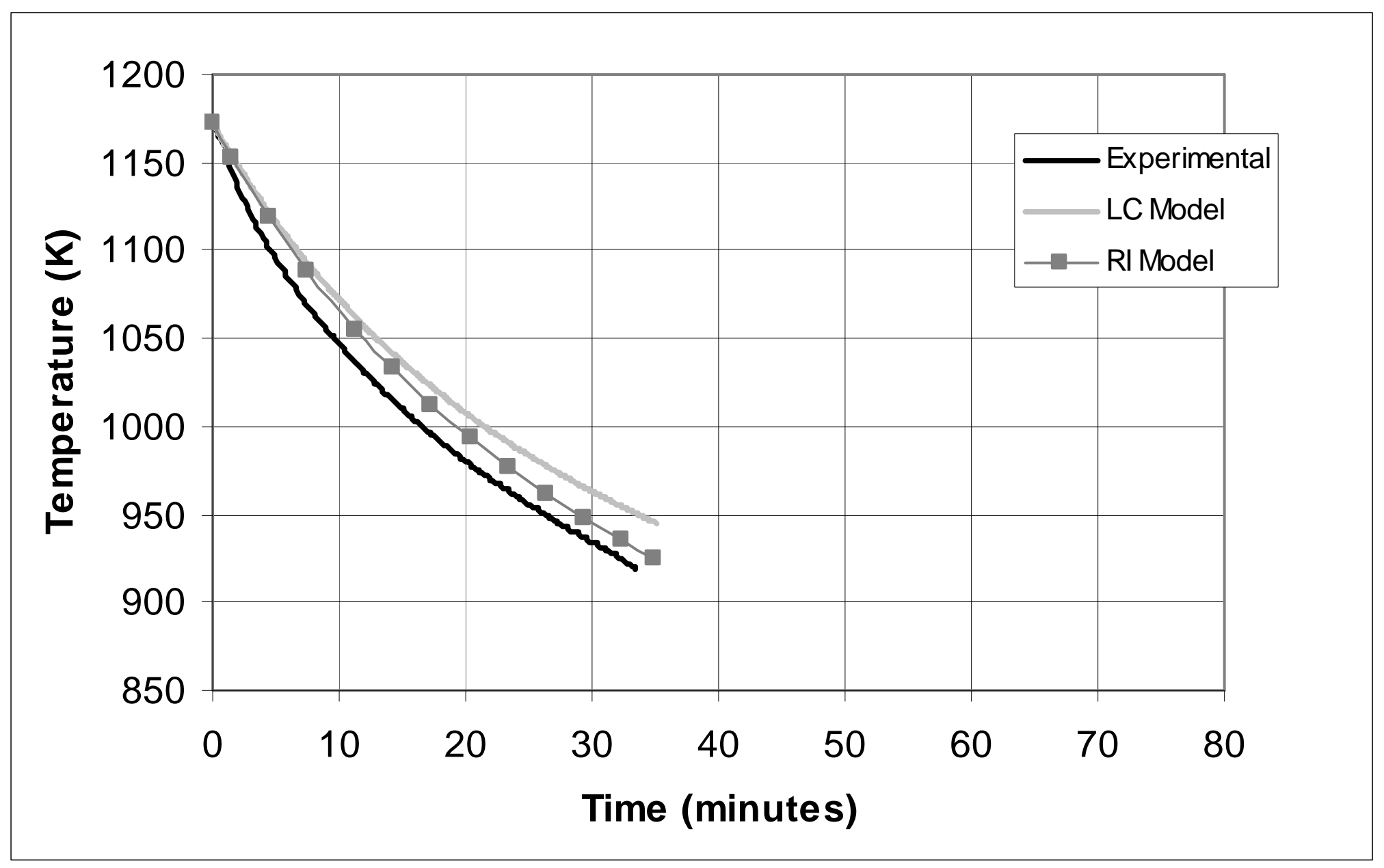

Figure 72 Average bed temperature during steam cooling (no ballast present) 


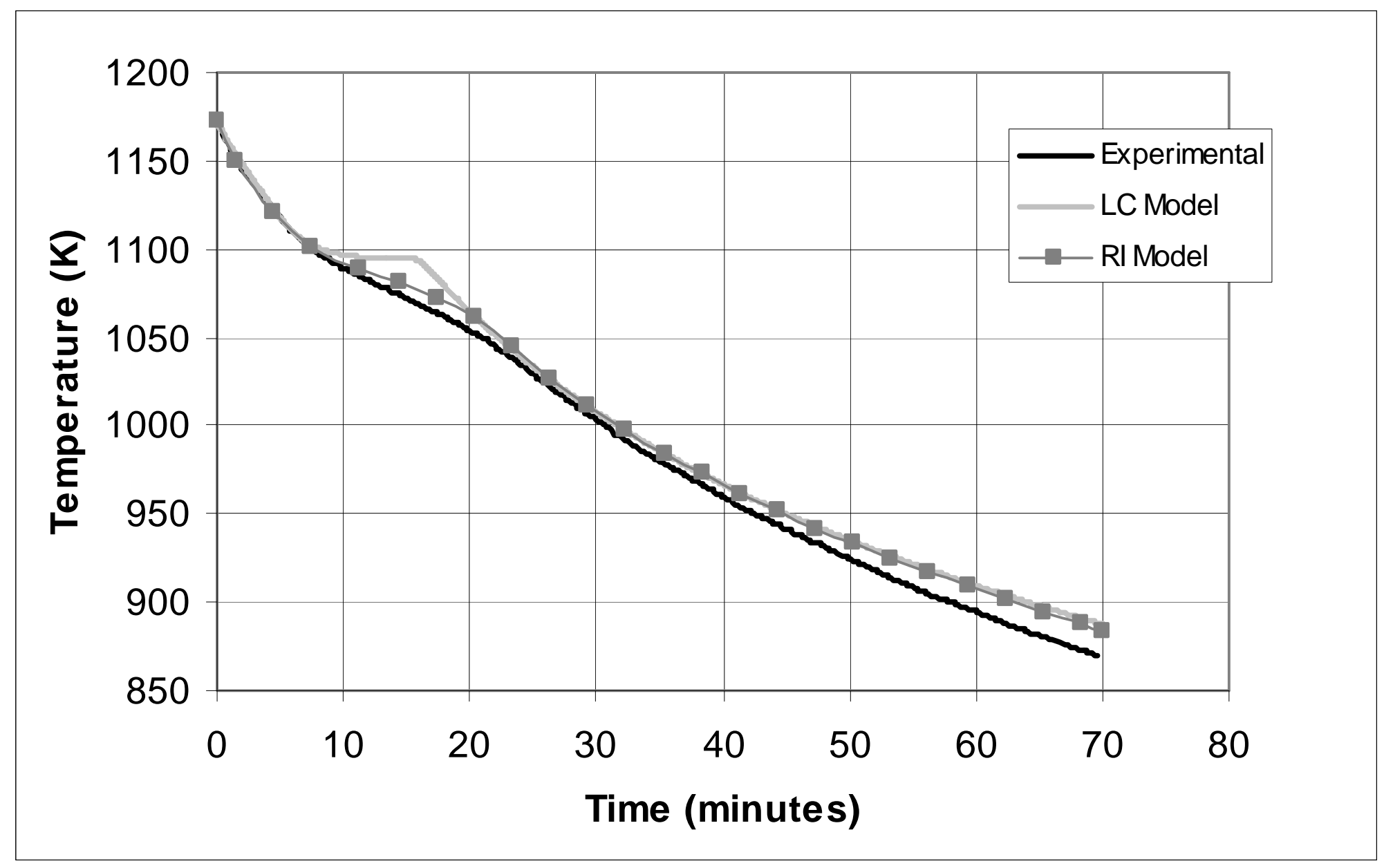

Figure 73 Average bed temperature during steam cooling (ballast present) 
Eq. 49

$$
t_{p c} \approx \frac{m_{p c} \cdot h_{p c, f u s}}{\dot{Q}_{\text {steam }}} .
$$

The purpose of the thermal ballast is to extend this period of minimal bed temperature loss to the greatest extent possible, in turn increasing the time that pyrolysis can be maintained in the reactor. The latent heat of fusion is fixed for a given phase-change material, so the only way to increase $t_{p c}$ for a given $\dot{Q}_{\text {steam }}$ is to increase the mass of phase-change material contained within the bed, $m_{p c}$.

The current ballast system consists of 48 ballast tubes containing $0.3 \mathrm{~kg}$ of lithium fluoride per tube, so the total mass of phase-change material was approximately $14.4 \mathrm{~kg}$. An additional 0.13 $\mathrm{kg}$ of lithium fluoride could be added to each tube while retaining the desired factor of safety with respect to internal tube pressures generated when the tubes are heated. In this case, the total amount of phase-change material would then increase to $20.9 \mathrm{~kg}$. An analysis was performed to determine the effect of this additional phase-change material. The resulting bed temperature profiles for this analysis are shown in Figure 74.

The phase-change time, $t_{p c}$, expected for the reactor containing $14.4 \mathrm{~kg}$ of lithium fluoride can be estimated from the heat transfer rate, $\dot{Q}_{\text {steam }}$, during the phase-change process. For a steam mass flowrate of $90.3 \mathrm{~kg} \mathrm{hr}^{-1}$ and an inlet temperature of $720 \mathrm{~K}, \dot{Q}_{\text {steam }}$ would have a value of approximately 79,600 kW. The latent heat of fusion of $\mathrm{LiF}, h_{p c, \text { fus }}$, has a value of $1050 \mathrm{~kJ} \mathrm{~kg}^{-1}$. Thus, for a reactor containing $14.4 \mathrm{~kg}$ of lithium fluoride, the phase-change time, $t_{p c}$, would be approximately 0.190 hours, or 11.4 minutes. For a reactor containing $20.9 \mathrm{~kg}$ of lithium fluoride, this time increases to approximately 0.275 hours, or 16.5 minutes.

From Figure 74, this time between the inflection points of the profile is approximately 13 minutes for a reactor containing $14.4 \mathrm{~kg}$ of phase-change material and approximately 18 minutes for a reactor containing $20.9 \mathrm{~kg}$ of phase-change material, which are in good agreement with the estimates obtained from Eq. 49. The slightly longer times predicted by the computer model reflects the contribution of sensible heat in the sand bed to the overall cooling process. The increase in cooling time is proportional to the increase in the mass of phase change material, in accordance with Eq. 49. The effect on cooling time persists even after solidification of the phase change material is complete.

\section{Sensitivity Analysis 2: Thermal conductivity of lithium fluoride}

As conduction through the solidified phase-change material plays a significant role on heat transfer between the bed and the ballast tubes during phase-change, thermal conductivity of the lithium fluoride was believed to be a critical parameter of the system. However, precise values of thermal conductivity were difficult to obtain for lithium fluoride. Tabulated data on a variety of phase-change materials listed the thermal conductivity of lithium fluoride in the range of 2.0 $4.0 \mathrm{~W} \mathrm{~m}^{-1} \mathrm{~K}^{-1}$. A value of $4.0 \mathrm{~W} \mathrm{~m}^{-1} \mathrm{~K}^{-1}$ was assumed for the solid-phase thermal conductivity of lithium fluoride, $k_{L i F}$, for the baseline model. However, another source reports thermal conductivity of lithium fluoride that is strongly temperature dependent, ranging from $6.0 \mathrm{~W} \mathrm{m-1}$ 
$\mathrm{K}^{-1}$ at $500 \mathrm{~K}$ to $3.0 \mathrm{~W} \mathrm{~m}^{-1} \mathrm{~K}^{-1}$ at $1100 \mathrm{~K}^{38}$. Therefore, using this data a sensitivity analysis was performed to determine the effect of the thermal conductivity of lithium fluoride on cooling curves. The results are given in Figure 75.

Slight variations exist during the phase-change period as the thermal conductivity of lithium fluoride ranges from $3.0 \mathrm{~W} \mathrm{~m}^{-1} \mathrm{~K}^{-1}$ to $6.0 \mathrm{~W} \mathrm{~m}^{-1} \mathrm{~K}^{-1}$. When the value of $k_{L i F}$ is $6.0 \mathrm{~W} \mathrm{~m}^{-1} \mathrm{~K}^{-1}$, the bed temperature remains at approximately $1070 \mathrm{~K}$ at the end of the phase-change process, while the bed temperature remains at approximately $1060 \mathrm{~K}$ at the end of the phase-change process when the value of $k_{L i F}$ is $3.0 \mathrm{~W} \mathrm{~m}^{-1} \mathrm{~K}^{-1}$. The phase-change time, $t_{p c}$, varies from approximately 11 minutes when $k_{L i F}$ is $6.0 \mathrm{~W} \mathrm{~m}^{-1} \mathrm{~K}^{-1}$ to 13 minutes when $k_{L i F}$ is $3.0 \mathrm{~W} \mathrm{~m}^{-1} \mathrm{~K}^{-1}$. However, these relatively modest effects do not persist through the full cooling curve, as the profiles converge quickly following the end of the phase-change process. Thus, the value of $k_{L i F}$ has little impact on the overall reactor behavior, particularly within the range of $3.0-6.0 \mathrm{~W} \mathrm{~m}^{-1}$ $\mathrm{K}^{-1}$.

Sensitivity Analysis 3: Characteristic dimension of the ballast

Transient heat transfer is heavily influenced by the Biot number of the thermal mass of the system, as defined by Eq. 24. The characteristic dimension $\mathrm{L}_{\mathrm{c}}$ is defined as the ratio of volume, $V$, and surface area, $A_{\text {surf }}{ }^{35}$ :

Eq. $50 \quad L_{c}=\frac{V}{A_{\text {surf }}}$.

For long cylinders, such as the ballast tubes, the characteristic dimension is equal to half the radius or one quarter of the diameter:

Eq. 51

$$
L_{c}=\frac{r_{\text {tube }}}{2}=\frac{d_{\text {tube }}}{4} .
$$

As the convection coefficient of the fluidized bed and the thermal conductivity within the ballast tube are relatively fixed, the only means of altering Biot number is to alter the characteristic dimension of the tubes. The actual diameter of the tubes was $2.54 \mathrm{~cm}$; sensitivity analysis was performed for ballast tube diameters of $1.27 \mathrm{~cm}, 2.54 \mathrm{~cm}$ and $5.08 \mathrm{~cm}$. In order to keep the amount of phase-change material constant across the various cases, however, the number of tubes and amount of lithium fluoride per tube was adjusted accordingly. The values of the parameters of this analysis are compiled in Table 39. The resulting fluidized bed temperature profiles are illustrated in Figure 76. The unballasted case has been included on Figure 76 to establish a baseline case.

The Biot number of each case can be calculated by assuming a convection coefficient, $h$, with a value of $300 \mathrm{~W} \mathrm{~m}^{-2} \mathrm{~K}^{-1}$, and a thermal conductivity, $k$, with a value of $4 \mathrm{~W} \mathrm{~m}^{-2} \mathrm{~K}^{-1}$. Three cases were evaluated with $L_{c}$ equal to $0.318 \mathrm{~cm}, 0.625 \mathrm{~cm}$, and $1.27 \mathrm{~cm}$, corresponding to Biot numbers of $0.238,0.476$, and 0.953 . None of these cases has small enough Biot number (less than 0.1 ) to treat the problem by lumped capacitance analysis, justifying the use of the computational model. 
The results show that the characteristic dimension, $L_{c}$, has a tremendous impact on cooling curves during the period of phase-change, although the effect is less dramatic once the phase change material has completely solidified. For a given mass of phase change material, smaller diameter tubes more closely approach the isothermal cooling curves of the idealized phase change process than larger tubes. There are two reasons for this result. First, the total surface area of the small diameter tubes is greater than that of the larger tubes. Second, the conduction path through solidified lithium fluoride is shorter for the smaller diameter tubes than for the larger tubes. The effect is large enough that the smallest diameter tubes evaluated had an essentially isothermal cooling curve over an interval of almost 10 minutes. The fluidized bed reaches a temperature of $900 \mathrm{~K}$ at approximately the same time in all three cases, but if nearisothermal behavior is desired during the phase-change process, the diameter of the ballast tubes should be kept as small as practical.

Table 39 Parameters examined in sensitivity analysis of $L_{c}$

\begin{tabular}{ccccccc}
\hline $\begin{array}{c}\text { Case } \\
\text { no. }\end{array}$ & $L_{c}(\mathrm{~cm})$ & $d_{\text {tube }}(\mathrm{cm})$ & $n_{\text {tubes }}$ & $m_{p c, \text { tube }}(\mathrm{kg})$ & $m_{p c \text {,total }}(\mathrm{kg})$ & Biot no. \\
\hline 1 & 0.3175 & 1.27 & 192 & 0.075 & 14.4 & 0.238 \\
2 & 0.6250 & 2.54 & 48 & 0.300 & 14.4 & 0.476 \\
3 & 1.2700 & 5.08 & 12 & 1.200 & 14.4 & 0.953 \\
\hline
\end{tabular}




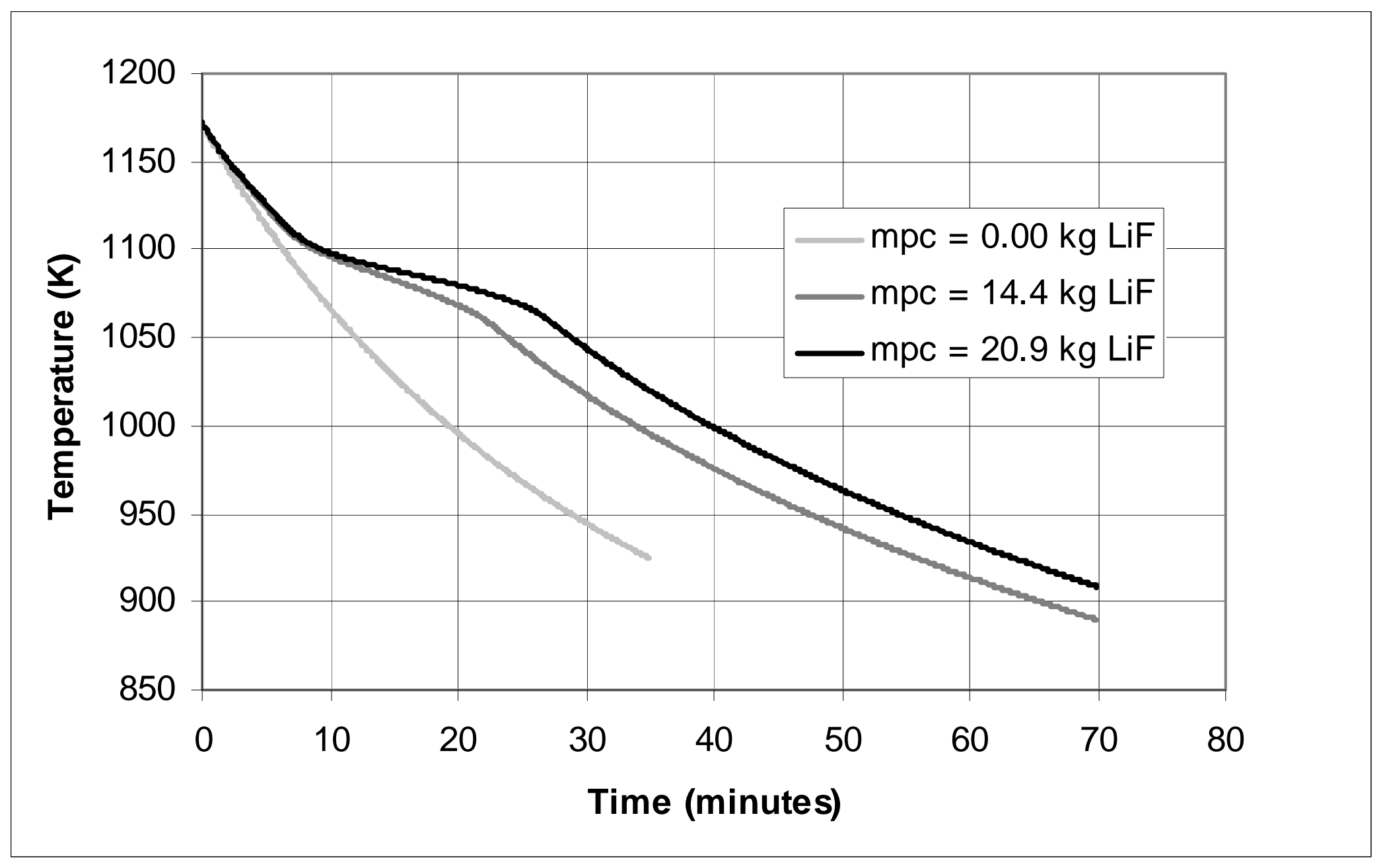

Figure 74 Bed temperature profiles for varied values of $m_{p c}$ 


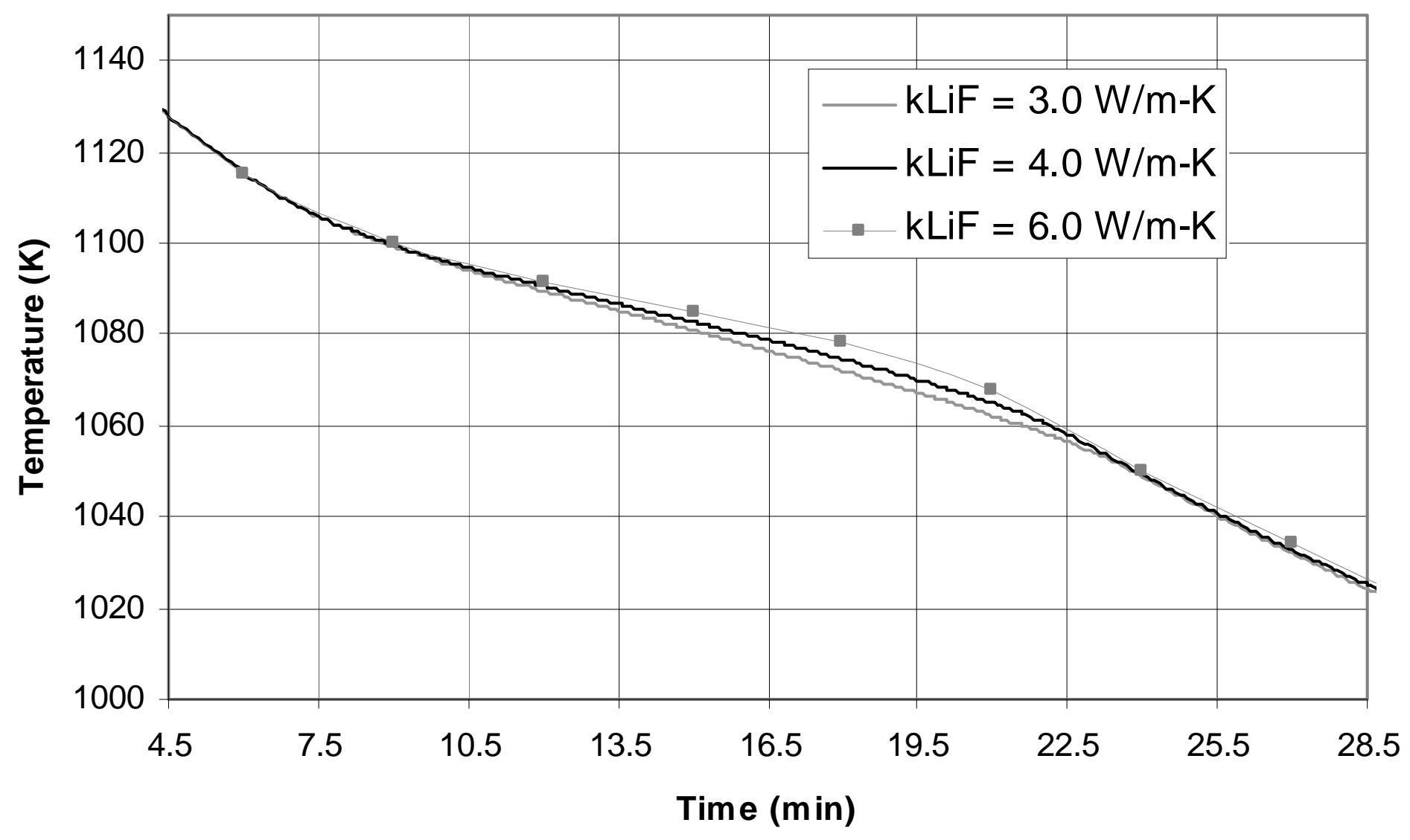

Figure 75 Bed temperature profiles for varied values of $k_{L i F}$ 


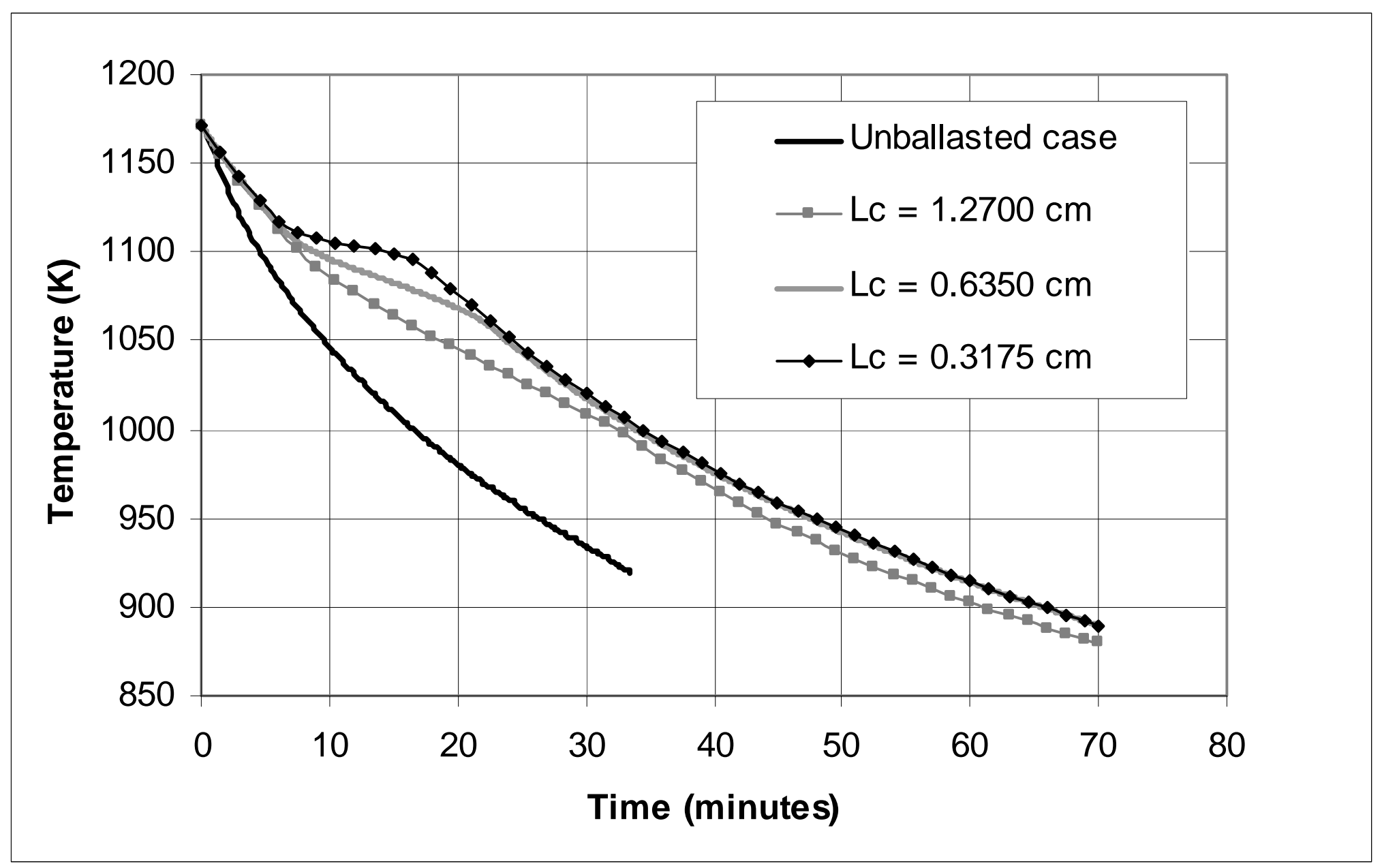

Figure 76 Bed temperature profiles for varied values of $L_{c}$ 


\section{Cost Estimating for Biomass Gasification Systems}

\subsection{Introduction}

Gasification technology has enjoyed tremendous development; however, its cost estimates have not developed at the same rate. Estimating the product cost of the gasification process plays the most important role in marketing the technology. Accordingly, our team is progressively receiving two major questions regarding the biomass gasification. The first question concerns the potential of such type of biomass to be converted to gas. The second question emphasizes the costs of biomass gasification equipment for applications ranging from byproduct disposal to the production of ammonia fertilizer. Our several years of experience in thermochemical conversion area and/or gasification literature help us to provide technical information on gasification technology. The diversity of feedstocks, applications, and plant sizes make it difficult to provide estimates of capital and operating costs for particular applications. In an effort to further cultivate interest by industry in biomass gasification, we propose to develop a methodology for rapidly estimating capital and operating costs.

Currently, the world consumes approximately $4.5 \times 10^{11}$ standard cubic meters of hydrogen annually having a market value of nearly $\$ 60$ billion (US dollars). Over $92 \%$ is used for refining and desulfurization of oil in refineries and the production of ammonia and methanol ${ }^{39}$. Over the next decade, hydrogen demand for current uses is expected to grow at double-digit rates ${ }^{40}$. A major demand not included in this prediction is the use of hydrogen as a fuel in the transportation economy. There are numerous means for production of hydrogen; however, the source of hydrogen is yet to be determined. Czernik ${ }^{41}$ reported that direct production of hydrogen from biomass by gasification/water-gas shift technology is economically unfavorable, except for very low cost feedstocks and very large plants. Other studies support the statement that the cost of production decreases with increasing plant sizes. Hydrogen production from biomass gasification will be explored more thoroughly throughout this section.

Determining the economics of hydrogen production from biomass gasification begins with detailed mass and energy balances on the proposed technology. The methodology will incorporate thermodynamic properties of important solids, gases, and liquids found in a gasification plant, but will not attempt to include chemical kinetic and transport properties required for dynamical simulation of the gasification process, which is not essential at this level of cost-estimating and, in many respects, is not well understood at present. Mass and energy balances will be used to appropriately size gasification equipment and estimate their capital cost. Since we will be dealing with a relatively small number of subsystems (solid fuel handling, gasification reactor, particulate removal equipment, tar removal system, trace contaminant removal systems, and gas conditioning) the inclusion of scaling relationships for different sizes of gasification plants will be relatively straightforward. Once the cost of capital equipment is estimated, a tabulation of operating costs can be made using calculated equations for various direct and indirect charges to the plant.

Although generalized process engineering software such as Aspen is available for such purposes, simpler and less expensive alternatives are desired for the current application. We proposed to use MathCad, a popular and easily mastered engineering analysis software tool, for performing 
the mass and energy balances as well as for the subsequent cost analyses. Therefore, the main objective of this task is to develop a simple and powerful tool for calculating the cost estimate for hydrogen production from biomass gasification systems. This methodology will build mainly upon standard cost estimating procedures adapted to bio-based manufacturing ${ }^{42}$. Specific objectives include:

(a) Perform a comprehensive mass and energy balances on the air blown gasification and ballasted gasification systems,

(b) Evaluate the total direct cost of hydrogen production from air blown gasification and ballasted gasification of biomass, and

(c) Predict the cost of a system producing 50 ton/day of hydrogen from a ballasted gasifier and an air blown gasifier.

\subsection{Methodology}

At Iowa State University, hydrogen has been produced by gasifying switchgrass and/or obsolete seed corn in a fluidized bed reactor and upgrading the gas using several catalytic reactors. We have developed a very capable fluidized bed gasifier that could be operated in various thermochemical modes including combustion, gasification/pyrolysis. The ratio of air to fuel determines the mode of operation. As was described in previous sections, in combustion mode the reactor is able to process approximately $20-60 \mathrm{~kg} / \mathrm{hr}$ of biomass. In gasification and pyrolysis modes the reactor is able to process $90-270 \mathrm{~kg} / \mathrm{hr}$. Detailed description of the fluidized bed gasifier as well as the catalytic reactors can be found in Section 2.

The fluidized bed gasifier located at Biomass Energy Conversion facility (BECON) in Nevada, Iowa was used to test the gasification of biomass feedstock under steady state conditions. A flow diagram of the biomass gasification system is shown Figure 77. It was tested under air blown gasification as well as ballasted gasification. Various measurements and controlling systems were incorporated to evaluate and/or control the performance of the fluidized bed gasification process. These include bed temperature, bed pressure drop, biomass feeding rate, air and steam flow rates, producer gas flow rate, gasification residues production rate, producer gas composition, contaminant concentration. Bed temperature was recorded every 10 seconds using type $\mathrm{K}$ thermocouples. An online Varian micro GC was used to analyze the composition of the producer gas every three minutes. For quality control, the same gas sample was analyzed using Continuous Emission Monitors (CEMs) to obtain the concentrations of $\mathrm{CO}, \mathrm{CO}_{2}, \mathrm{H}_{2}$ and $\mathrm{O}_{2}$. The CEMs are capable of analyzing a sample every 10 seconds. The fluidized bed gasifier is fully computerized including the control system as well as the data acquisition systems.

\section{$\underline{\text { Direct Costs }}$}

The first step in estimating the cost of hydrogen production is to determine the cost of equipment to be installed in the plant. Air supply system, steam supply system, biomass feed system, gas conditioning system, control system, gas cleaning are among some of the more common items. Two module factors namely material module factor (MMF) and labor module factor (LMF) are necessary to be included in the cost estimate calculations. MMF is defined as the ratio of the cost of materials to install a particular piece of equipment to the cost of the equipment. LMF is defined as the ratio of the cost of labor to install a particular piece of equipment to the combined 


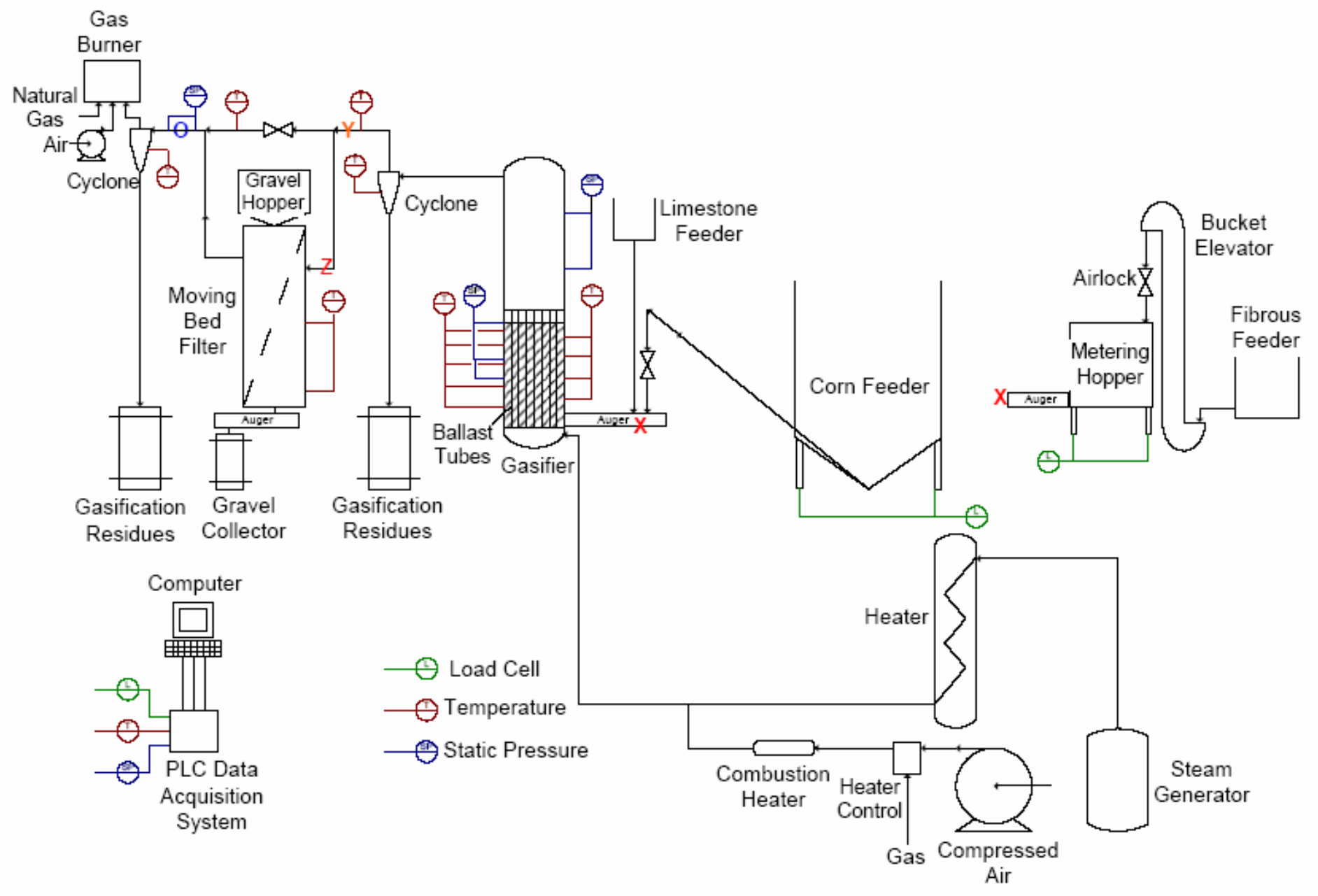

Biomass Gasification Flow Diagram

Figure 77 A flow diagram for the fluidized bed gasification system 
cost of the installed equipment and materials used to perform the installation.

Eq. 52

$$
\mathrm{C}_{\mathrm{M}}=\mathrm{MMF}^{*} \mathrm{C}_{\mathrm{P}}
$$

where:

$$
\begin{array}{ll}
\mathrm{C}_{\mathrm{M}} & \text { is the material cost, } \\
\mathrm{MMF} & \text { is the tabulated material module factor, and } \\
\mathrm{C}_{\mathrm{P}} & \text { is the price of the equipment. }
\end{array}
$$

Eq. 53

$$
\mathrm{C}_{\mathrm{L}}=\mathrm{LMF} *\left(\mathrm{C}_{\mathrm{P}}+\mathrm{C}_{\mathrm{M}}\right)
$$

where:
$\mathrm{C}_{\mathrm{L}}$
is the labor cost, and
$\mathrm{LMF}$
is the tabulated labor module factor.

The direct cost of purchasing and installing equipment is the summation of the equipment cost, materials cost and labor costs as shown in the following equation:

Eq. 54

$$
\mathrm{C}_{\mathrm{D}}=\mathrm{C}_{\mathrm{P}}+\mathrm{C}_{\mathrm{M}}+\mathrm{C}_{\mathrm{L}}
$$

where $C_{D}$ is the direct cost.

\section{$\underline{\text { Indirect Costs }}$}

Indirect costs are expenses associated with the construction of a plant or facility that cannot be characterized as equipment, materials or labor. The three most common indirect costs are categorized as (a) fringe, insurance and taxes, (b) construction overhead and (c) engineering expenses. The fringe, insurance, taxes can be calculated as follows:
Eq. 55
$\mathrm{C}_{\mathrm{FIT}}=0.08 * \mathrm{C}_{\mathrm{P}}$

where $\mathrm{C}_{\text {FIT }}$ is the fringe, insurance and taxes cost. The construction overhead can be calculated as follows:

Eq. 56

$$
\mathrm{C}_{\mathrm{O}}=0.7 * \mathrm{C}_{\mathrm{L}}
$$

where $\mathrm{C}_{\mathrm{O}}$ is the construction overhead cost. The engineering expenses can be calculated as follows:

Eq. 57

$$
\mathrm{C}_{\mathrm{E}}=0.15 *\left(\mathrm{C}_{\mathrm{P}}+\mathrm{C}_{\mathrm{M}}\right)
$$

where $C_{E}$ is the engineering cost.

The total indirect cost can be calculated by adding the previous items as shown in the following equation:

Eq. 58

$$
\mathrm{C}_{\mathrm{ID}}=\mathrm{C}_{\mathrm{FIT}}+\mathrm{C}_{\mathrm{O}}+\mathrm{C}_{\mathrm{E}}
$$


where $\mathrm{C}_{\mathrm{ID}}$ is the total direct cost. The bare module cost is defined as the combination of the direct and indirect costs:

Eq. 59

$$
\mathrm{C}_{\mathrm{BM}}=\mathrm{C}_{\mathrm{D}}+\mathrm{C}_{\mathrm{ID}}
$$

where $\mathrm{C}_{\mathrm{BM}}$ is the bare module cost.

\section{Other Costs}

There are two other costs associated with the gasification project including the contingency and fee as well as the auxiliary facilities. The contingency and fee can be calculated as follows:

Eq. 60

$$
\mathrm{C}_{\mathrm{CF}}=0.18 * \mathrm{C}_{\mathrm{BM}}
$$

where $\mathrm{C}_{\mathrm{CF}}$ is the contingency and fee costs. While the auxiliary cost can be calculated as follows:

Eq. 61

$$
\mathrm{C}_{\mathrm{AF}}=0.3 *\left(\mathrm{C}_{\mathrm{BM}}+\mathrm{C}_{\mathrm{CF}}\right)
$$

where $\mathrm{C}_{\mathrm{AF}} \quad$ is the auxiliary facilities cost.

The grassroots capital is the summation of the contingency and fee and the auxiliary facilities as follows:

Eq. 62

$$
\mathrm{C}_{\mathrm{GR}}=\mathrm{C}_{\mathrm{BM}}+\mathrm{C}_{\mathrm{CF}}+\mathrm{C}_{\mathrm{AF}}
$$

where $\mathrm{C}_{\mathrm{GR}}$ is the grassroots costs.

\section{Operating Costs}

Operating costs include direct, indirect costs and annual operating costs. Indirect costs include raw material cost, operating labor, supervisory labor, maintenance and repair cost, operating supplies, laboratory charges, patents and royalties. Indirect expenses include the overhead, local taxes, insurance and general expenses. Brown ${ }^{42}$ reported the detailed calculations of the operating costs.

Annual capital charge is the cost to pay off the loan as follows:

Eq. 63

$$
A_{c c}=\frac{P_{i} i(1+i)^{N}}{\left[(1+i)^{N}-1\right]}
$$

where:
Acc is the annual capital charge
$\mathrm{P}_{\mathrm{i}} \quad$ is the loan principal amount
$\mathrm{i} \quad$ is the interest rate
$\mathrm{N} \quad$ is the number of years for loan payment 


\section{Accounting for Economies of Scale}

A balanced basis of comparison must scale capital costs to practical equipment size. The predicted cost of specified equipment is proportional to the known cost of baseline equipment multiplied by the ratio of the size of the specified equipment to the size of the base line equipment to the power of the scale-sizing exponent.

Eq. 64

$$
\mathrm{C}_{\mathrm{p}, \mathrm{S}}=\mathrm{C}_{\mathrm{p}, \mathrm{b}}\left(\frac{\mathrm{S}_{\mathrm{s}}}{\mathrm{S}_{\mathrm{b}}}\right)^{\mathrm{n}}
$$

where:

$$
\begin{array}{ll}
\mathrm{C}_{\mathrm{p}, \mathrm{S}} & \text { is the predicted cost of specific equipment } \\
\mathrm{C}_{\mathrm{p}, \mathrm{b}} & \text { is the known cost of baseline equipment } \\
\mathrm{S}_{\mathrm{s}} & \text { is the size of the specified equipment } \\
\mathrm{S}_{\mathrm{b}} & \text { is the size of the baseline equipment } \\
\mathrm{n} & \text { is the economy of scale sizing exponent }(<1)
\end{array}
$$

\subsection{Air Blown Gasification}

\section{Mass Balance for Air Blown Gasification}

Four experimental runs were completed to test the mass balance during the air blown gasification. Air was used as a gasifying agent and switchgrass or obsolete corn seeds were used as a feedstock. Char was collected using two cyclones and weighed. Tar was collected using a pressure condenser filled with a coil of santoprene tubing. The tubing weight was determined before and after the test to determine the tar weight. The producer gas flow rate was measured using a pitot tube. The producer gas compositions were determined using the Varian micro-GC as well as the Continuous Emission Monitors to validate the gas compositions.

Ten thermocouples were positioned in various locations within the fluidized bed gasifier. The average bed temperature was recorded every 10 seconds. Average bed temperature was stable at approximately $730 \pm 10{ }^{\circ} \mathrm{C}$ during the steady state operation. The gasifier was kept in a steady state condition for several hours. An S-type pitot tube using the U.S. Environmental Protection Agency's Method 2 with National Institute of Standards and Technology traceable standards was used to measure the flow rate of the producer gas. This method is applicable for quantifying gas flow using an S-type pitot tube. The differential pressure from the pitot tube was converted to a flow rate and standardized in some calculations using the pitot tube temperature. The volumetric gas flow rate reached approximately $6.5 \mathrm{Nm}^{3} / \mathrm{min}$. The gas heating values associated with the gas stream output was approximately $5.2 \mathrm{MJ} / \mathrm{m}^{3}$. Catalyzing the producer gas enhanced the hydrogen concentration by more than three folds. The raw gas contains $8.8 \%$ hydrogen (vol. \%, dry basis) while the gas exiting the low temperature shift reactor has a hydrogen concentration of $28.7 \%$.

The input mass balance was calculated during the steady state gasification using the load cells for corn seeds and the mass flow meter for the air as well as rotometers for the purged air. Producer gas flow rate was measured using the pitot tube. Producer gas temperature was recorded at the pitot tube location to calculate the normalized gas rate. 
Table 40 shows the input and output mass flow rates in addition to the mass balance as percentage. The overall mass balance reached $107.2 \pm 6.0 \%$. The lower values of the input materials compared to the output materials could be due to the accuracy of the rotometers used to measure the purged air. Our results showed that the hydrogen production after catalysts reached $0.067 \mathrm{~kg} / \mathrm{kg}$ of biomass gasified. Bowen ${ }^{43}$ reported that hydrogen production could reach 0.08 $\mathrm{kg} / \mathrm{kg}$ biomass.

Table 41 shows the carbon, nitrogen, oxygen, and hydrogen balances during the steady state gasification tests. The elemental balances shown in the table verify the accuracy of the results and support the claim that the mass balance is accurate. The inputs are from corn and air with the outputs of the elements from output water, tar, char, and gas components read by the Varian micro-GC.

\section{Energy Balance for Air Blown Gasification}

Based on the input feed rate of corn seeds and their heating value, the input fuel energy was calculated and recorded as shown in Table 42. Tar energy, char energy, heat of vaporization, chemical enthalpy and sensible enthalpy are the five items taken into account to calculate the output energy. Energy balance during the steady state gasification reached $83.6 \pm 5.2 \%$. Energy loss through the reactor walls and piping is the major contribution for the remaining energy loss leading to an energy balance less than $100 \%$.

\section{Cost Estimate for Hydrogen from Air Blown Gasification}

It was essential to start the cost estimate on the pilot plant gasifier located at BECON to have a baseline of the equipment costs, capital costs and operating costs. The cost estimate was performed using the developed program. Equipment costs, material for installation costs, direct labor for installation of equipment, and total direct costs are summarized in Table 43, which shows that the total direct costs of $\$ 352,800$.

\subsection{Ballasted Gasification}

Ballasted gasification is a dynamic process with various changeable parameters. As described previously, the latent heat ballasting system consists of an array of stainless steel tubes enclosing high temperature phase-change material namely lithium fluoride. Several experiments were conducted to test the quality of the producer gas resulting from the ballast gasification process and to perform a mass and energy balances on the system. 
Table 40 Mass balance during air blown gasification

\begin{tabular}{|c|c|c|c|c|c|c|c|c|c|c|c|c|c|c|c|}
\hline \multicolumn{3}{|c|}{ Input $(\mathrm{kg} / \mathrm{hr})$} & \multicolumn{13}{|c|}{ Output $(\mathrm{kg} / \mathrm{hr})$} \\
\hline$\stackrel{\Xi}{\Xi}$ & 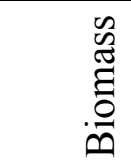 & : & 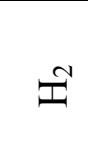 & ర̃ & $\mathbf{Z}^{2}$ & $\stackrel{\bigcirc}{\circlearrowright}$ & Õ & 焉 & $\underset{U^{N}}{\mathbb{N}}$ & $\underset{\widetilde{U}}{\stackrel{N}{J}}$ & $\underset{\widetilde{U}}{\mathcal{U}^{\circ}}$ & $\underbrace{\infty}$ & $\begin{array}{l}\underset{N}{I} \\
\underset{I}{0}\end{array}$ & 突 & $\stackrel{\vec{\sigma}}{\oplus}$ \\
\hline Avg. & 191.3 & 275.1 & 2.3 & 0.1 & 238 & 64.5 & 128 & 10.3 & 9.0 & $*$ & 1.2 & * & 31.5 & 10.1 & 4.9 \\
\hline St.Dev. & 9.3 & 19.6 & 0.1 & 0.1 & 13.9 & 6.3 & 7.7 & 0.7 & 1.0 & $*$ & 0.4 & $*$ & 1.3 & 2.5 & 2.2 \\
\hline Total & \multicolumn{2}{|c|}{$466.4 \pm 27.2$} & \multicolumn{13}{|c|}{$499.9 \pm 28.7$} \\
\hline Balance & \multicolumn{15}{|c|}{$107.2 \% \pm 6.0 \%$} \\
\hline
\end{tabular}


Table 41 Elemental balances during the air blown gasification

\begin{tabular}{lcccccccc}
\hline & \multicolumn{2}{c}{ Carbon } & \multicolumn{2}{c}{ Nitrogen } & \multicolumn{2}{c}{ Oxygen } & \multicolumn{2}{c}{ Hydrogen } \\
\hline Item & $\begin{array}{c}\text { In } \\
(\mathrm{kg} / \mathrm{hr})\end{array}$ & $\begin{array}{c}\text { Out } \\
(\mathrm{kg} / \mathrm{hr})\end{array}$ & $\begin{array}{c}\text { In } \\
(\mathrm{kg} / \mathrm{hr})\end{array}$ & $\begin{array}{c}\text { Out } \\
(\mathrm{kg} / \mathrm{hr})\end{array}$ & $\begin{array}{c}\text { In } \\
(\mathrm{kg} / \mathrm{hr})\end{array}$ & $\begin{array}{c}\text { Out } \\
(\mathrm{kg} / \mathrm{hr})\end{array}$ & $\begin{array}{c}\text { In } \\
(\mathrm{kg} / \mathrm{hr})\end{array}$ & $\begin{array}{c}\text { Out } \\
(\mathrm{kg} / \mathrm{hr})\end{array}$ \\
\hline Avg. & 76.0 & 86.4 & 216.9 & 237.9 & 155.0 & 158.2 & 12.6 & 10.9 \\
St.Dev. & 3.7 & 6.1 & 15.4 & 13.8 & 8.2 & 10.0 & 0.6 & 0.4 \\
\hline
\end{tabular}

Table 42 Energy balance during air blown gasification

\begin{tabular}{lccccc}
\hline & Input $(\mathrm{kW})$ & \multicolumn{3}{c}{ Output $(\mathrm{kW})$} \\
\hline Item & Fuel Energy & $\begin{array}{c}\text { Chemical } \\
\text { Enthalpy }\end{array}$ & $\begin{array}{c}\text { Sensible } \\
\text { Enthalpy }\end{array}$ & Tar Energy & Char Energy \\
\hline Avg. \pm St. Dev. & $917.1 \pm 44.7$ & $568.6 \pm 32.9$ & $113.3 \pm 4.9$ & $46.4 \pm 20.9$ & $38.4 \pm 9.5$ \\
\hline Total & $917.1 \pm 44.7$ & \multicolumn{4}{c}{$766.7 \pm 45.7$} \\
\hline Energy Balance & & \multicolumn{3}{c}{$83.6 \pm 5.2 \%$} \\
\hline
\end{tabular}


Table 43 Equipment costs, material for installation, direct labor for installation, and total direct costs for air blown gasification

\begin{tabular}{|l|r|r|r|r|}
\hline \multicolumn{1}{|c|}{ Item } & \multicolumn{1}{c|}{$\begin{array}{c}\text { Cost } \\
\mathbf{( \$ )}\end{array}$} & $\begin{array}{r}\text { Materials for installation } \\
\mathbf{( \$ )}\end{array}$ & $\begin{array}{c}\text { Direct labor } \\
\mathbf{( \$ )}\end{array}$ & \multicolumn{1}{c|}{$\begin{array}{c}\text { Total direct } \\
(\mathbf{\$})\end{array}$} \\
\hline Air supply system $^{*}$ & 4,000 & 800 & 1,300 & 6,100 \\
\hline Biomass feed system $^{*}$ & 7,000 & 1,400 & 2,300 & 10,700 \\
\hline Gasifier $^{* * *}$ & 35,000 & 7,000 & 11,300 & 53,300 \\
\hline Gas conditioning system $^{*}$ & 125,000 & 25,000 & 40,500 & 190,500 \\
\hline Control system $^{*}$ & 23,000 & 4,600 & 7,500 & 35,100 \\
\hline Hydrogen separation ** & 44,000 & 8,800 & 4,300 & 57,100 \\
\hline Total & $\mathbf{2 3 8 , 0 0 0}$ & $\mathbf{4 7 , 6 0 0}$ & $\mathbf{6 7 , 2 0 0}$ & $\mathbf{3 5 2 , 8 0 0}$ \\
\hline
\end{tabular}

${ }^{*}$ Numbers obtained from vendors based on prices at 2004.

** Numbers estimated from Quest Air Technologies Inc. (personal communications)

***Data estimated from Ghosh et. al. ${ }^{44}$ 
Figure 78 and Table 44 show the variations in the average bed temperatures during the combustion and pyrolysis phases. It can be seen that temperature rose from $650{ }^{\circ} \mathrm{C}$ to $770{ }^{\circ} \mathrm{C}$ (char burn out) within $7 \mathrm{~min}$ with the heating rate of $40.0^{\circ} \mathrm{C} / \mathrm{min}$. During the combustion phase, temperature rose to $900{ }^{\circ} \mathrm{C}$ with the heating rate of $13{ }^{\circ} \mathrm{C} / \mathrm{min}$. During pyrolysis phase, the temperature reduced to $650{ }^{\circ} \mathrm{C}$ within $6 \mathrm{~min}$, with the cooling rate of $35.7^{\circ} \mathrm{C} / \mathrm{min}$. Changes in the heating and cooling rates depend mainly on the weight of the bed material used assuming that all other conditions stayed unchangeable. The bed temperature did not exceed $900{ }^{\circ} \mathrm{C}$ to prevent agglomeration and was kept above $650{ }^{\circ} \mathrm{C}$ to reduce the tar production.

Gas composition during the pyrolysis phase was recorded every 10 seconds using the CEMs and every three minutes using the micro-GC. Table 45 summarizes the peak value of the gas compositions during combustion phase. As expected during the combustion phase that nitrogen content will be the highest followed by carbon dioxide. During the pyrolysis phase, carbon monoxide and hydrogen increased to $33 \%$ and $31 \%$, respectively as described in the previous sections. Similarly, methane and ethylene increased to $10 \%$ and $7.4 \%$, respectively. On the other hand, non-combustible gas concentrations decreased to their lower values during the pyrolysis phase.

\section{Mass Balance for Ballasted Gasification}

Table 46 shows the producer gas flow rate during combustion-pyrolysis phases measured during four cycles. Average flow rate during pyrolysis is $2.3 \mathrm{Nm}^{3} / \mathrm{min}$ with a standard deviation of 0.2 $\mathrm{Nm}^{3} / \mathrm{min}$. The flow rates during char burnout and combustion were higher than that of pyrolysis stages. The flow rates during char burnout and combustion averaged $5.0 \mathrm{Nm}^{3} / \mathrm{min}$ and 4.7 $\mathrm{Nm}^{3} /$ min with standard deviations of $0.4 \mathrm{Nm}^{3} / \mathrm{min}$ and $0.3 \mathrm{Nm}^{3} / \mathrm{min}$ respectively. The heating value associated with the producer gas during the pyrolysis stage reached $8.0 \mathrm{MJ} / \mathrm{m}^{3}$.

Producer gas slipstream was passed through four catalytic reactors including guard bed, steam reforming reactor, high temperature shift reactor and low temperature shift reactor. The function of these reactors was to destruct ammonia and hydrogen sulfide by the guard bed, crack tar to lower molecular weight hydrocarbons by the steam-reforming reactor. High and low temperature reactors played the role of enhancing hydrogen content using the gas shift reaction. Hydrogen content in the raw producer gas was $24.0 \%$ and doubled after the steam reforming reactor thereafter, it increased by two and half folds after the high and low temperature shift reactor. Figure 79 shows the hydrogen concentration after passing through the low temperature gas shift reaction during two cycles. The reverse results were observed with $\mathrm{CO}$, which decreased from $39.1 \%$ to $23.3 \%$ after the steam-reforming reactor and $11.6 \%$ after the high temperature shift reactor and $0.9 \%$ after the low temperature shift reactor (Figure 80 ). This confirms that the gas shift reaction took place.

Mass balance was performed on the pilot plant ballasted gasifier. Measured inputs included biomass feed rate and flow rates of steam, air, and nitrogen into the gasifier. Measured outputs included flow rates of flue gas and producer gas, char and tar production rates, water content. Table 47 shows the mass balance measured during four cycles. To simplify the operation of the gasifier, corn was used as a feedstock during combustion phase while switchgrass was used during pyrolysis phase. The average mass balance during the cycles reached $105.7 \pm 3.3 \%$. 
Table 48 shows the carbon, nitrogen, oxygen, and hydrogen balances during ballasted gasification tests, which supports the claim that the mass balance is accurate.

\section{Energy Balance for Ballasted Gasification}

An energy balance was performed on the ballasted fluidized bed gasifier as shown in Table 49, which was found to be $90.3 \% \pm 5.2 \%$. Ballast gasification has a cold gas efficiency of $56.6 \%$ with a standard deviation of $2.4 \%$. This result is comparable to energy balances performed on conventional gasification trials. The reasons for the incomplete closure on energy balances could be due to (a) the elevated dynamics during the cycles, (b) the use of nitrogen during the pyrolysis phase instead of purged air to cool the auger, (c) switching between two feedstocks during the two phases, and (d) heat losses through reactor.

\section{Cost Estimate for Hydrogen from Ballasted Gasification}

The same cost analysis technique was used to calculate the cost of producing hydrogen using the ballasted gasifier. Once again, it was essential to establish a baseline of cost estimate of the ballast system based on an inclusive mass and energy balance. This baseline estimation will be used to scale up the system at any required level. In addition to the air supply system, biomass feed system, reactor, gas conditioning system, control system and gas cleaning system; ballasted gasification technique required a steam supply system and a ballasted system. Table 50 shows equipment cost, materials for installation, direct labor, and total direct costs for a ballasted biomass gasification. Since pyrolysis operates in the absence of air, steam is used to fluidize the bed. The costs of a boiler, steam control valve, steam flow meter, and superheaters as well as the ballast tubes have been added to the equipment cost. It should be noted that the equipment cost was used to calculate other variables, which will have an effect on the cost of production more than just the increase in equipment cost. Total capital cost for the pilot-scale, ballasted gasifier is estimated to be $\$ 390,900$, which is $11 \%$ higher than the capital cost for the pilot-scale, air-blown gasifier.

Catalyzing the producer gas during the pyrolysis phase increased the hydrogen production rate to $4.5 \mathrm{~kg} / \mathrm{h}$. Several improvements could be made on the ballasted system by adding another reactor, which in turn will increase the hydrogen production rate. Scaling up the ballasted gasification system to produce double or triple the amount of hydrogen will certainly reduce the production cost. Reduction of the energy loss could increase the pyrolysis duration which in turn will add to the hydrogen production. 


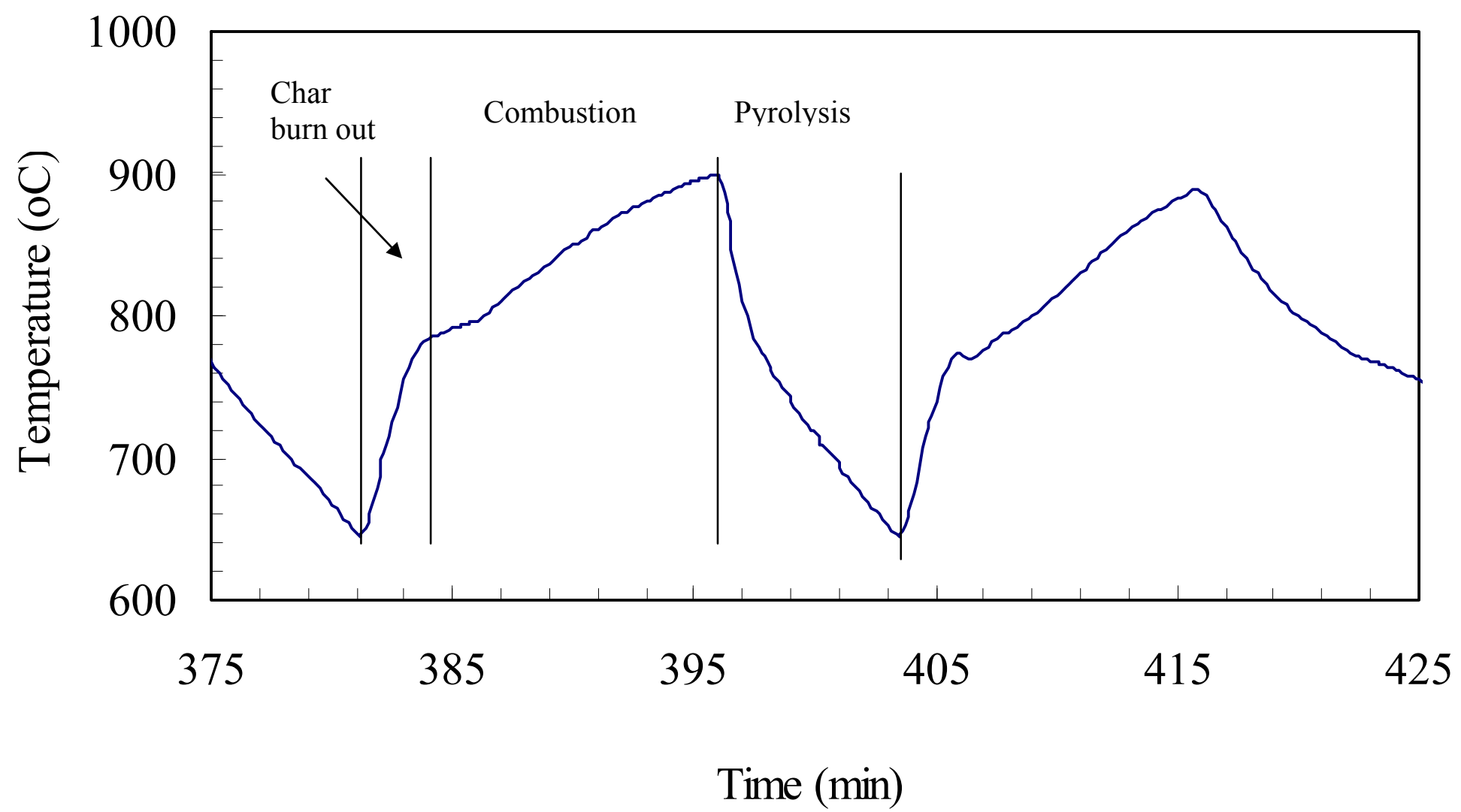

Figure 78 Temperature cycles during combustion-pyrolysis phases 


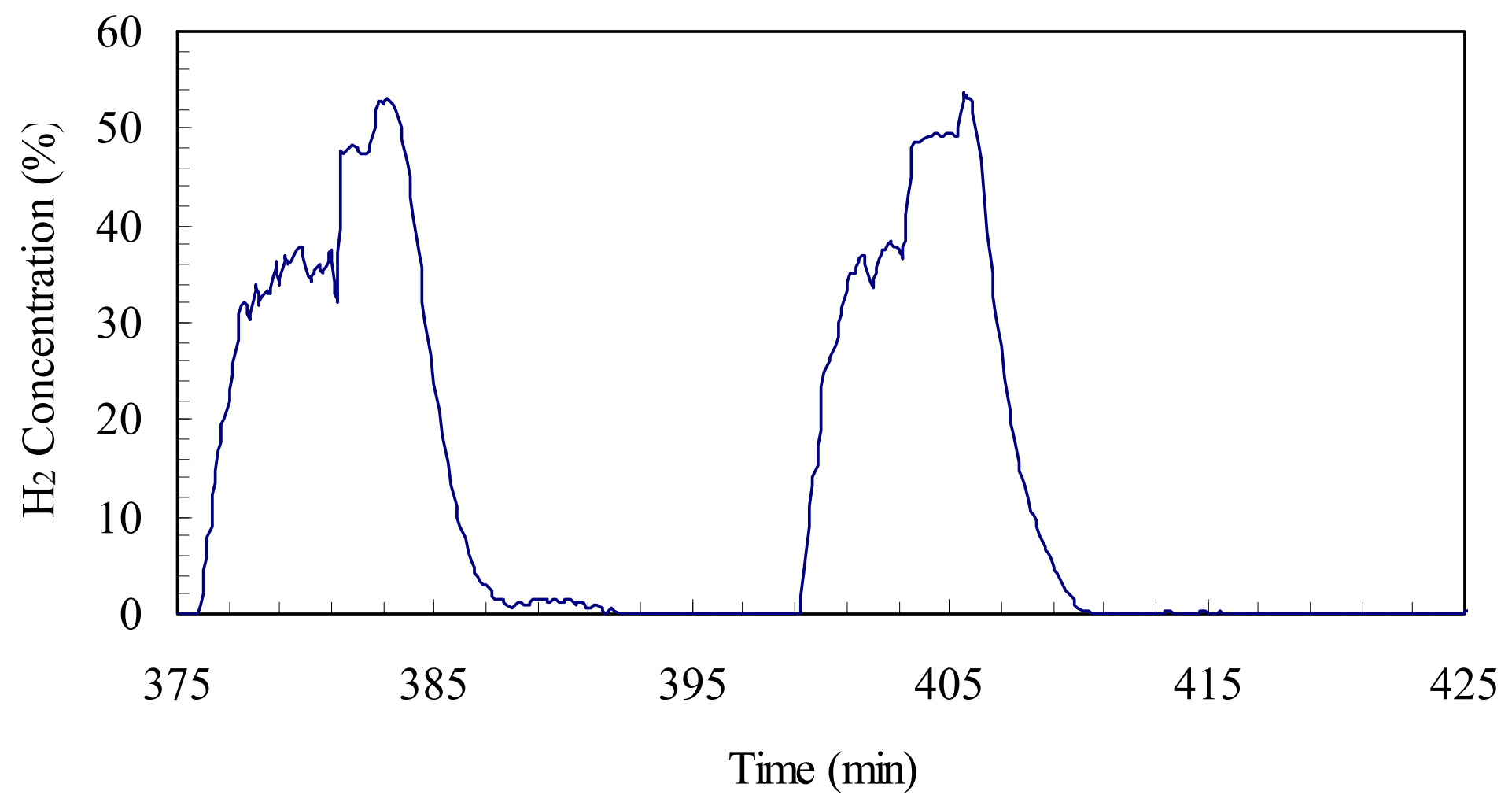

Figure 79 Hydrogen cycle exiting the low temperature shift reactor 
Table 44 Temperature changes during the combustion and pyrolysis phases

\begin{tabular}{|l|c|c|c|}
\hline Phase & Pyrolysis & Char burn out & Combustion \\
\hline $\begin{array}{l}\text { Heating and cooling } \\
\text { temperatures }\left({ }^{\circ} \mathrm{C}\right)\end{array}$ & $900 \rightarrow 650$ & $650 \rightarrow 770$ & $770 \rightarrow 900$ \\
\hline Duration $(\mathrm{min})$ & 7 & 3 & 10 \\
\hline $\begin{array}{l}\text { Heating and/or cooling } \\
\text { rate }\left({ }^{\circ} \mathrm{C} / \mathrm{min}\right)\end{array}$ & 35.7 & 40.0 & 13.0 \\
\hline
\end{tabular}

Table 45 Peak gas composition (\%) during the combustion phase

\begin{tabular}{|l|c|c|c|c|}
\hline & \multicolumn{3}{|c|}{ Gas composition (\%) } & \\
\hline Cycle No. & $\mathbf{O}_{\mathbf{2}}$ & $\mathbf{N}_{\mathbf{2}}$ & $\mathbf{C O}_{\mathbf{2}}$ & $\mathbf{C O}$ \\
\hline Cycle 1 & 0.3 & 71.5 & 17.7 & 0.2 \\
\hline Cycle 2 & 0.5 & 71.6 & 17.4 & 0.2 \\
\hline Cycle 3 & 0.2 & 71.2 & 17.6 & 0.2 \\
\hline Cycle 4 & 0.4 & 71.9 & 17.7 & 0.3 \\
\hline Average & 0.4 & 71.6 & 17.6 & 0.2 \\
\hline St. Dev & 0.1 & 0.3 & 0.1 & 0.1 \\
\hline
\end{tabular}

Table 46 Producer gas flow rate during combustion-pyrolysis phases

\begin{tabular}{|l|c|c|c|c|c|c|}
\hline & \multicolumn{7}{|c|}{ Gas flow rate* $\left.\mathbf{( N m}^{\mathbf{3}} / \mathbf{m i n}\right)$} \\
\hline Cycle phase & Cycle 1 & Cycle 2 & Cycle 3 & Cycle 4 & Average & St. Dev. \\
\hline Pyrolysis & 2.3 & 2.1 & 2.6 & 2.4 & 2.3 & 0.2 \\
\hline Char Burnout & 5.8 & 4.7 & 4.7 & 5.0 & 5.0 & 0.4 \\
\hline Combustion & 5.1 & 4.9 & 4.4 & 4.6 & 4.7 & 0.3 \\
\hline
\end{tabular}

*Dry standard condition 


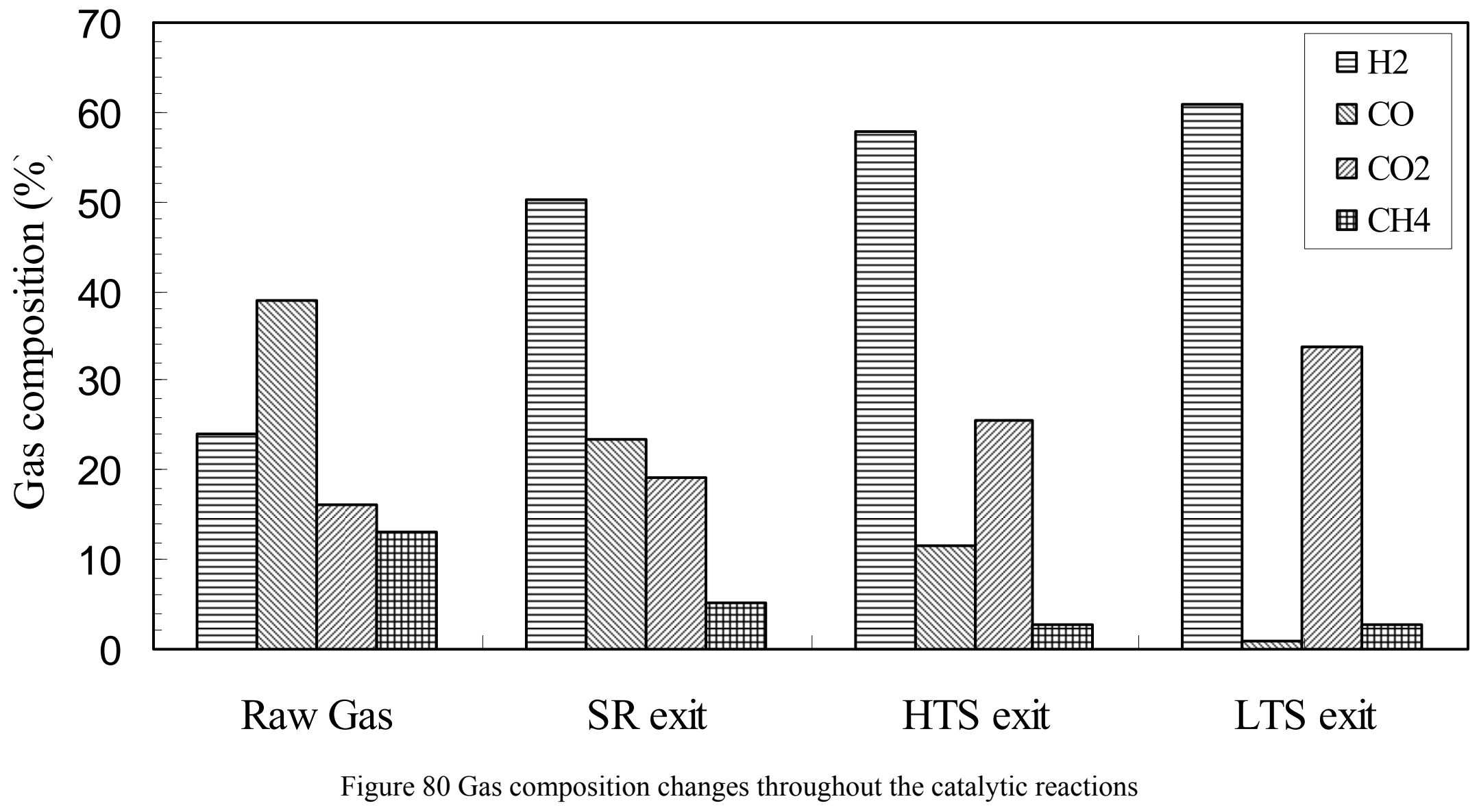


Table 47 Material balances during ballasted gasification

\begin{tabular}{|c|c|c|c|c|c|c|c|c|c|c|c|c|c|c|c|c|c|c|}
\hline \multicolumn{6}{|c|}{ Input (kg/cycle) } & \multicolumn{13}{|c|}{ Output (kg/cycle) } \\
\hline 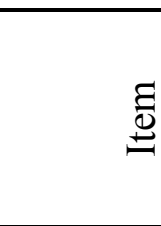 & $\begin{array}{l}\text { n } \\
\text { है } \\
\text { है } \\
.0\end{array}$ & 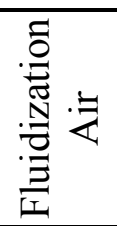 & 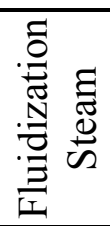 & $\begin{array}{l}\mathcal{Z} \\
0 \\
0 \\
\Xi \\
\Xi\end{array}$ & 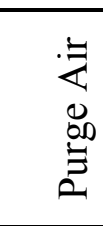 & $\mathcal{I}$ & $0^{2}$ & $Z^{N}$ & O & $\delta$ & 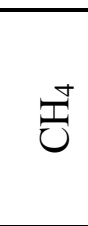 & $\vec{J}_{U}^{+}$ & $\underset{\mathcal{U}}{\widetilde{I}}$ & $\frac{I^{N}}{U^{\prime}}$ & $\underset{U}{\stackrel{\infty}{m}}$ & $\underset{I}{0}$ & 荀 & $\stackrel{\bar{E}}{\epsilon}$ \\
\hline Avg. & 38.2 & 67.3 & 10.1 & 9.6 & 23.7 & 0.5 & 0.3 & 79.7 & 8.6 & 36.4 & 1.9 & 1.4 & 0.2 & 0.1 & 0.4 & 22.5 & 5.3 & 0.1 \\
\hline St. Dev. & 2.1 & 4.3 & 0.2 & 0.2 & 1.5 & 0.0 & 0.1 & 4.0 & 0.5 & 2.0 & 0.1 & 0.1 & 0.0 & 0.0 & 0.0 & 0.7 & 1.1 & 0.0 \\
\hline
\end{tabular}

Table 48 Elemental balances during ballasted gasification

\begin{tabular}{lcccccccc}
\hline & \multicolumn{2}{c}{ Carbon } & \multicolumn{2}{c}{ Nitrogen } & \multicolumn{2}{c}{ Oxygen } & \multicolumn{2}{c}{ Hydrogen } \\
\hline Item & $\begin{array}{c}\text { In } \\
(\mathrm{kg} / \mathrm{cycle})\end{array}$ & $\begin{array}{c}\text { Out } \\
(\mathrm{kg} / \mathrm{cycle})\end{array}$ & $\begin{array}{c}\text { In } \\
(\mathrm{kg} / \mathrm{cycle})\end{array}$ & $\begin{array}{c}\text { Out } \\
(\mathrm{kg} / \mathrm{cycle})\end{array}$ & $\begin{array}{c}\text { In } \\
(\mathrm{kg} / \mathrm{cycle})\end{array}$ & $\begin{array}{c}\text { Out } \\
(\mathrm{kg} / \mathrm{cycle})\end{array}$ & $\begin{array}{c}\text { In } \\
(\mathrm{kg} / \mathrm{cycle})\end{array}$ & $\begin{array}{c}\text { Out } \\
(\mathrm{kg} / \mathrm{cycle})\end{array}$ \\
\hline Avg. & 16.5 & 18.9 & 78.5 & 79.6 & 43.4 & 51.7 & 3.1 & 4.2 \\
St.Dev. & 0.9 & 1.2 & 4.3 & 4.0 & 2.0 & 2.0 & 0.1 & 0.2 \\
\hline
\end{tabular}


Table 49 Energy balance during ballasted gasification

\begin{tabular}{|c|c|c|c|c|c|c|}
\hline & \multicolumn{2}{|c|}{ Input (MJ) } & \multicolumn{4}{|c|}{ Output (MJ) } \\
\hline Item & $\begin{array}{c}\text { Fuel } \\
\text { Energy }\end{array}$ & $\begin{array}{c}\text { Steam } \\
\text { Sensible } \\
\text { Enthalpy }\end{array}$ & $\begin{array}{l}\text { Chemical } \\
\text { Enthalpy }\end{array}$ & $\begin{array}{l}\text { Sensible } \\
\text { Enthalpy }\end{array}$ & $\begin{array}{c}\text { Tar } \\
\text { Energy }\end{array}$ & Char Energy \\
\hline Avg. \pm St. Dev. & $641.1 \pm 35.1$ & $13.1 \pm 0.1$ & $362.8 \pm 17.8$ & $150.2 \pm 5.5$ & $4.9 \pm 1.7$ & $72.7 \pm 16.0$ \\
\hline Total & \multicolumn{2}{|c|}{$654.2 \pm 35.1$} & \multicolumn{4}{|c|}{$590.6 \pm 37.2$} \\
\hline Energy Balance & \multicolumn{6}{|c|}{$90.3 \pm 5.2 \%$} \\
\hline
\end{tabular}

Table 50 Equipment costs, material for installation, and direct labor for installation during ballasted gasification

\begin{tabular}{|l|r|r|r|r|}
\hline & \multicolumn{1}{|c|}{$\begin{array}{c}\text { Cost } \\
\text { Item }\end{array}$} & $\begin{array}{r}\text { Materials for installation } \\
\mathbf{( \$ )}\end{array}$ & $\begin{array}{c}\text { Direct labor } \\
\mathbf{( \$ )}\end{array}$ & $\begin{array}{c}\text { Total direct } \\
\mathbf{( \$ )}\end{array}$ \\
\hline Air supply system $^{*}$ & 4,000 & 800 & 1,300 & 6,100 \\
\hline Steam supply system $^{*}$ & 19,000 & 3,800 & 6,200 & 29,000 \\
\hline Biomass feed system $^{*}$ & 7,000 & 1,400 & 2,300 & 10,700 \\
\hline Gasifier $^{* * *}$ & 35,000 & 7,000 & 11,300 & 53,300 \\
\hline Gas conditioning system $^{*}$ & 125,000 & 25,000 & 40,500 & 190,500 \\
\hline Control system $^{*}$ & 23,000 & 4,600 & 7,500 & 35,100 \\
\hline Hydrogen separation* & 44,000 & 8,800 & 4,300 & 57,100 \\
\hline Ballast system* & 6,000 & 1,200 & 1,900 & 9,100 \\
\hline Total & $\mathbf{2 6 3 , 0 0 0}$ & $\mathbf{5 2 , 6 0 0}$ & $\mathbf{7 5 , 3 0 0}$ & $\mathbf{3 9 0 , 9 0 0}$ \\
\hline
\end{tabular}

"Data obtained from venders based on prices at 2004.

** Data estimated from Quest Air Technologies Inc. (personal communications)

***Data estimated from Ghosh et. al. ${ }^{44}$ 


\section{Cost Estimate for 50 ton/day Hydrogen Production}

The cost estimate developed in the previous sections of the air-blown gasification as well as ballasted gasification was used to calculate the equipments cost, capital cost and operating cost of a gasifier able to produce 50 ton/day of hydrogen. These calculations were used to estimate the hydrogen production cost from an air-blown gasifier as well as a ballasted gasifier. Mass balance was used to calculate air flow rate, steam flow rate (in the case of ballasted gasifier) and biomass feed rate required to produce the 50 ton/day hydrogen. Several assumptions were taken into account to calculate the equipment costs, capital costs and operating costs including: (a) biomass is available all year around with the cost of $\$ 55 /$ ton, (b) twenty four workers are required to run and manage the facility with the average salary of $\$ 25 / \mathrm{h}$, (c) the interest rate is $10 \%$ with the number of loan years is 20 years, (d) no tax break was taken into account, (e) the scaling up method, which uses a baseline of a pilot-scale system, applies to this large of a scale, which assumes a scaling exponent of 0.6 for non tabulated items.

Table 51, Table 52 and

Table 53 the cost estimates of producing 50-ton hydrogen per day from an air blown gasification system. The total direct cost, total capital cost and the annual operating cost were $8.74,19.46$, and 32.03 million dollars, respectively. Daily biomass feed rate to the ballasted gasifier, which produces the 50-ton/day of hydrogen is $746 \mathrm{ton} /$ day. The cost estimate of a system producing 50-ton/day of hydrogen is projected to be $\$ 1.85 / \mathrm{kg} \mathrm{H}_{2}$. 
Table 51 Equipment costs, material for installation, direct labor for installation of equipment, and total direct costs for 50 ton/day hydrogen production from air blown gasifier

\begin{tabular}{|l|c|c|c|c|c|}
\hline \multicolumn{1}{|c|}{ Item } & $\begin{array}{c}\text { Sizing } \\
\text { exponent } \\
(-)\end{array}$ & $\begin{array}{c}\text { Free on } \\
\text { board } \\
\text { cost } \\
\mathbf{( 1 0 0 0 \$ )}\end{array}$ & $\begin{array}{c}\text { Materials for } \\
\text { installation } \\
\mathbf{( 1 0 0 0 \$ )}\end{array}$ & $\begin{array}{c}\text { Direct } \\
\text { labor } \\
(\mathbf{1 0 0 0} \$)\end{array}$ & $\begin{array}{c}\text { Total } \\
\text { direct } \\
(\mathbf{1 0 0 0})\end{array}$ \\
\hline Air supply system & $0.68^{*}$ & 128 & 26 & 41 & 195 \\
\hline Steam supply system & $0.50^{*}$ & 0 & 0 & 0 & 0 \\
\hline Biomass feed system & $0.68^{*}$ & 224 & 45 & 72 & 341 \\
\hline Gasifier & $0.71^{*}$ & 1,302 & 260 & 422 & 1,984 \\
\hline Gas conditioning system & $0.60^{* *}$ & 2,656 & 531 & 861 & 4,048 \\
\hline Control system & $0.60^{* *}$ & 489 & 98 & 158 & 745 \\
\hline Hydrogen separation & $0.60^{* *}$ & 935 & 187 & 303 & 1425 \\
\hline Ballast system & $0.60^{* *}$ & 0 & 0 & 0 & 0 \\
\hline Total & & $\mathbf{5 , 7 3 4}$ & $\mathbf{1 , 1 4 7}$ & $\mathbf{1 , 8 5 7}$ & $\mathbf{8 , 7 3 8}$ \\
\hline
\end{tabular}

* Source: Brown ${ }^{42}$

** $\quad$ Numbers obtained using the six-tenth rule 
Table 52 Summary of capital costs for 50 ton/day hydrogen production from air blown gasifier

\begin{tabular}{|l|c|}
\hline \multicolumn{1}{|c|}{ Item } & $\begin{array}{c}\text { Cost } \\
\text { (million\$) }\end{array}$ \\
\hline Direct project expenses & \\
\hline Equipment (free on board) & 5.73 \\
\hline Materials for installation & 1.15 \\
\hline Direct labor & 1.86 \\
\hline Total direct & 8.74 \\
\hline Indirect project expenses & \\
\hline Freight, insurance, taxes & 0.46 \\
\hline Overhead & 1.30 \\
\hline Engineering expenses & 1.03 \\
\hline Total indirect & 2.79 \\
\hline Bare module cost & 11.53 \\
\hline Contingency \& fee & 2.08 \\
\hline Total module cost & 13.61 \\
\hline Auxiliary facilities & 4.08 \\
\hline Grassroots capital & 17.69 \\
\hline Working capital & 1.77 \\
\hline Total capital & 19.46 \\
\hline
\end{tabular}


Table 53 Summary of operating costs for 50

ton/day hydrogen production from air

blown gasifier

\begin{tabular}{|l|r|}
\hline \multicolumn{1}{|c|}{ Item } & \multicolumn{1}{l|}{$\begin{array}{l}\text { Cost } \\
\text { (million\$) }\end{array}$} \\
\hline Direct & 14.23 \\
\hline Raw materials & 4.99 \\
\hline Operating labor & 0.75 \\
\hline Supervisory labor & 1.42 \\
\hline Maintenance and repair & 0.21 \\
\hline Operating supplies & 0.75 \\
\hline Laboratory charges & 0.67 \\
\hline Patents and royalties & 23.02 \\
\hline Direct expenses & \\
\hline Indirect and general expenses & 4.29 \\
\hline Overhead & 0.35 \\
\hline Local Taxes & 0.18 \\
\hline Insurance & 1.90 \\
\hline General Expenses & 6.72 \\
\hline Indirect subtotal & 2.28 \\
\hline Annual capital charges & 32.03 \\
\hline Annual operating cost & 1.85 \\
\hline Production cost (\$/kgH $\mathbf{2})$ &
\end{tabular}

Table 54, Table 55, and Table 56 show the cost estimate of a 50 ton/day hydrogen production ballasted gasification system. The total direct cost, total capital cost and the annual operating cost were 14.37, 31.99, and 42.06 million dollars, respectively. The hydrogen production cost showed $\$ 2.43 / \mathrm{kg}$ hydrogen. This is $31 \%$ higher than that of air blown gasification. Air blown gasification has a higher concentration of nitrogen in the raw gas stream than that of ballasted gasification, which results in an increase in catalytic bed material and ultimately the cost of the system. Nitrogen is also one of the most difficult gases for a PSA to remove. Hydrogen purification using one PSA could be impractical with volumetric hydrogen concentrations less than 25\%. Two PSA's are necessary for air blown gasification as well as ballasted gasification. However, higher final concentrations of hydrogen will result from ballasted gasification due to higher PSA inlet concentrations. The purification of hydrogen is increased with higher volumetric hydrogen input streams. Despite this, the hydrogen production level is less than that of air blown gasification due to lower biomass feeding. Extra equipment is also needed for ballasted gasification which adds to a significantly higher capital cost. These two factors lead to a higher hydrogen production cost of $\$ 0.58$ for ballasted gasification over air-blown gasification. 
This hydrogen production cost is less than the value determined by the Delvine (2004) ${ }^{45}$. He reported that hydrogen production from biomass at plant gate by the year 2003 to be $\$ 6.10 / \mathrm{kg}$. Spath et al. (2003) ${ }^{46}$ assessed the economic feasibility of producing hydrogen from biomass via gasification process for the Battelle/FERCO and the IGT gasifier systems. They reported that the plant gate hydrogen selling price is lowest for the Battelle/FERCO gasifier plant (\$1.99$2.41 / \mathrm{kg} \mathrm{H}_{2}$ for a $15 \%$ after-tax internal rate of return), followed by the IGT gasifier system (\$2.27- 2.98/ $/ \mathrm{kg} \mathrm{H}_{2}$ for a $15 \%$ after-tax internal rate of return). Futher updates to the Battelle gasifier used for an economic analysis by Spath et al. (2005) ${ }^{47}$ show $\$ 1.38 / \mathrm{kg} \mathrm{H}_{2}$ in 2002 dollars using a $10 \%$ after-tax internal rate of return.

Simbeck and Chang (2002) ${ }^{48}$ compared the costs of hydrogen produced from a 150 ton/day central plant based on biomass as well as other materials such as natural gas, coal and water. They suggested the cost of hydrogen would be in the range of $\$ 2-3.5 / \mathrm{kg} \mathrm{H}_{2}$. The results showed that increasing the hydrogen production rate decreased the hydrogen production cost. However, there is a point where increasing the capacity can start to increase in hydrogen production costs. This is due to the fact that biomass is a major economic aspect of the annual operating costs. Biomass is also assumed to be readily available with transportation costs slightly increasing. Transportation fees can be costly and once the biomass is consumed in the immediate area, the plant will have to find biomass from other regions, which will add to the cost. The 50 ton/day economic analysis may be reaching this limit. Labor cost is also key role factor in the cost estimate and is a variable that is at the judgment of the plant owners. The assumptions used are reasonable and it is further demonstrated that increasing the plant capacity will decrease the cost of hydrogen production. Therefore, the hydrogen enhancement is mandatory to improve its cost. In addition to the reactor improvements, a tax break for hydrogen production from biomass thermochemical conversion system could help in enhancing these technologies.

The DOE target price for hydrogen produced from biomass is $\$ 2.90 / \mathrm{kg} \mathrm{H}_{2}$ by 2010 , dropping to $\$ 2.00 / \mathrm{kg} \mathrm{H}$ by 2015 . Thus, the ballasted gasifier, estimated to produce hydrogen at $\$ 2.43 / \mathrm{kg}$ $\mathrm{H}_{2}$, is able to meet the 2015 target within the uncertainty of the analysis $( \pm 30 \%)$. Significant decreases in the cost of biomass feedstock will have the largest impact on decreasing the hydrogen production cost.

The MathCad program was used to predict the hydrogen production cost under various biomass cost, labor cost and number of loan years for air-blown gasification. A base case close to the optimum cost conditions was used and each variable was independently changed around its base value in the range shown in Table 57. The effects of the biomass cost, labor cost and number of loan years on the hydrogen production cost are presented in Figure 81, Figure 82 and Figure 83. The cost of biomass appeared to be the most significant operating parameter influencing the hydrogen production cost. Increasing the biomass cost from $\$ 30$ to $\$ 80 /$ ton increased the hydrogen production cost by $\$ 0.81 / \mathrm{kg}$. Increasing the labor cost from $\$ 15 / \mathrm{h}$ to $\$ 35 / \mathrm{h}$ increased the hydrogen production cost from $\$ 1.59 / \mathrm{kg}$ to $\$ 2.11 / \mathrm{kg}$.

Figure 83 shows the sensitivity analysis for varying loan terms. This graph represents annual hydrogen production cost for the term of the loan which is shown to be a factor that does not have an influential role on the cost of hydrogen production. Hydrogen production cost after the loan term has finished is $\$ 1.72 / \mathrm{kg}$. 
Table 54 Equipment costs, material for installation, and direct labor for installation of equipment, and total direct costs for 50 ton/day hydrogen production from a ballasted gasifier

\begin{tabular}{|l|c|c|c|c|c|}
\hline \multicolumn{1}{|c|}{ Item } & $\begin{array}{c}\text { Sizing } \\
\text { exponent } \\
\mathbf{( - )}\end{array}$ & $\begin{array}{c}\text { Free on } \\
\text { board cost } \\
\mathbf{( 1 0 0 0 \$ )}\end{array}$ & $\begin{array}{c}\text { Materials for } \\
\text { installation } \\
\mathbf{( 1 0 0 0 \$ )}\end{array}$ & $\begin{array}{c}\text { Direct } \\
\text { labor } \\
\mathbf{( 1 0 0 0 \$ )}\end{array}$ & $\begin{array}{c}\text { Total } \\
\text { direct } \\
\mathbf{( 1 0 0 0 \$ )}\end{array}$ \\
\hline Air supply system & $0.68^{*}$ & 260 & 52 & 84 & 396 \\
\hline Steam supply system & $0.50^{*}$ & 409 & 82 & 133 & 624 \\
\hline Biomass feed system & $0.68^{*}$ & 455 & 91 & 147 & 693 \\
\hline Gasifier & $0.71^{*}$ & 2,737 & 547 & 887 & 4,171 \\
\hline Gas conditioning system & $0.60^{* *}$ & 3,265 & 653 & 1,058 & 4,976 \\
\hline Control system & $0.60^{* *}$ & 915 & 183 & 296 & 1,394 \\
\hline Hydrogen separation & $0.60^{* *}$ & 1,149 & 230 & 372 & 1,751 \\
\hline Ballast system & $0.60^{* *}$ & 239 & 48 & 77 & 364 \\
\hline Total & & $\mathbf{9 , 4 2 9}$ & $\mathbf{1 , 8 8 6}$ & $\mathbf{3 , 0 5 4}$ & $\mathbf{1 4 , 3 6 9}$ \\
\hline
\end{tabular}

* Source: Brown ${ }^{42}$

** Numbers obtained using the six-tenth rule 
Table 55 Summary of capital costs for 50 ton/day hydrogen production from a ballasted gasifier

\begin{tabular}{|l|c|}
\hline \multicolumn{1}{|c|}{ Item } & $\begin{array}{c}\text { Cost } \\
\text { (million\$) }\end{array}$ \\
\hline Direct project expenses & \\
\hline Equipment (free on board) & 9.43 \\
\hline Materials for installation & 1.89 \\
\hline Direct labor & 3.05 \\
\hline Total direct & 14.37 \\
\hline Indirect project expenses & \\
\hline Freight, insurance, taxes & 0.75 \\
\hline Overhead & 2.14 \\
\hline Engineering expenses & 1.70 \\
\hline Total indirect & 4.59 \\
\hline Bare module cost & 18.96 \\
\hline Contingency \& fee & 3.41 \\
\hline Total module cost & 22.37 \\
\hline Auxiliary facilities & 6.71 \\
\hline Grassroots capital & 29.08 \\
\hline Working capital & 2.91 \\
\hline Total capital & 31.99 \\
\hline
\end{tabular}


Table 56 Operating costs for 50 ton/day hydrogen production from the ballasted gasifier

\begin{tabular}{|l|r|}
\hline \multicolumn{1}{|c|}{ Item } & \multicolumn{1}{l|}{$\begin{array}{l}\text { Cost } \\
\text { (million\$) }\end{array}$} \\
\hline Direct & 20.28 \\
\hline Raw materials & 4.99 \\
\hline Operating labor & 0.75 \\
\hline Supervisory labor & 2.33 \\
\hline Maintenance and repair & 0.35 \\
\hline Operating supplies & 0.75 \\
\hline Laboratory charges & 0.88 \\
\hline Patents and royalties & 30.33 \\
\hline Direct expenses & \\
\hline Indirect and general expenses & 4.84 \\
\hline Overhead & 0.58 \\
\hline Local Taxes & 0.29 \\
\hline Insurance & 2.27 \\
\hline General Expenses & 7.98 \\
\hline Indirect subtotal & 3.76 \\
\hline Annual capital charges & 42.06 \\
\hline Annual operating cost & 2.43 \\
\hline Production cost (\$/kgH $\mathbf{2}$ ) & \\
\hline
\end{tabular}

Table 57 Range of variables used for the hydrogen cost predictions

Variable

Biomass cost

Labor cost

Number of loan

years
Unit

$\$ /$ ton

$\$ / \mathrm{h}$

$(-)$
Base

55

25

20
Range

$30-80$

$15-35$

10-30 


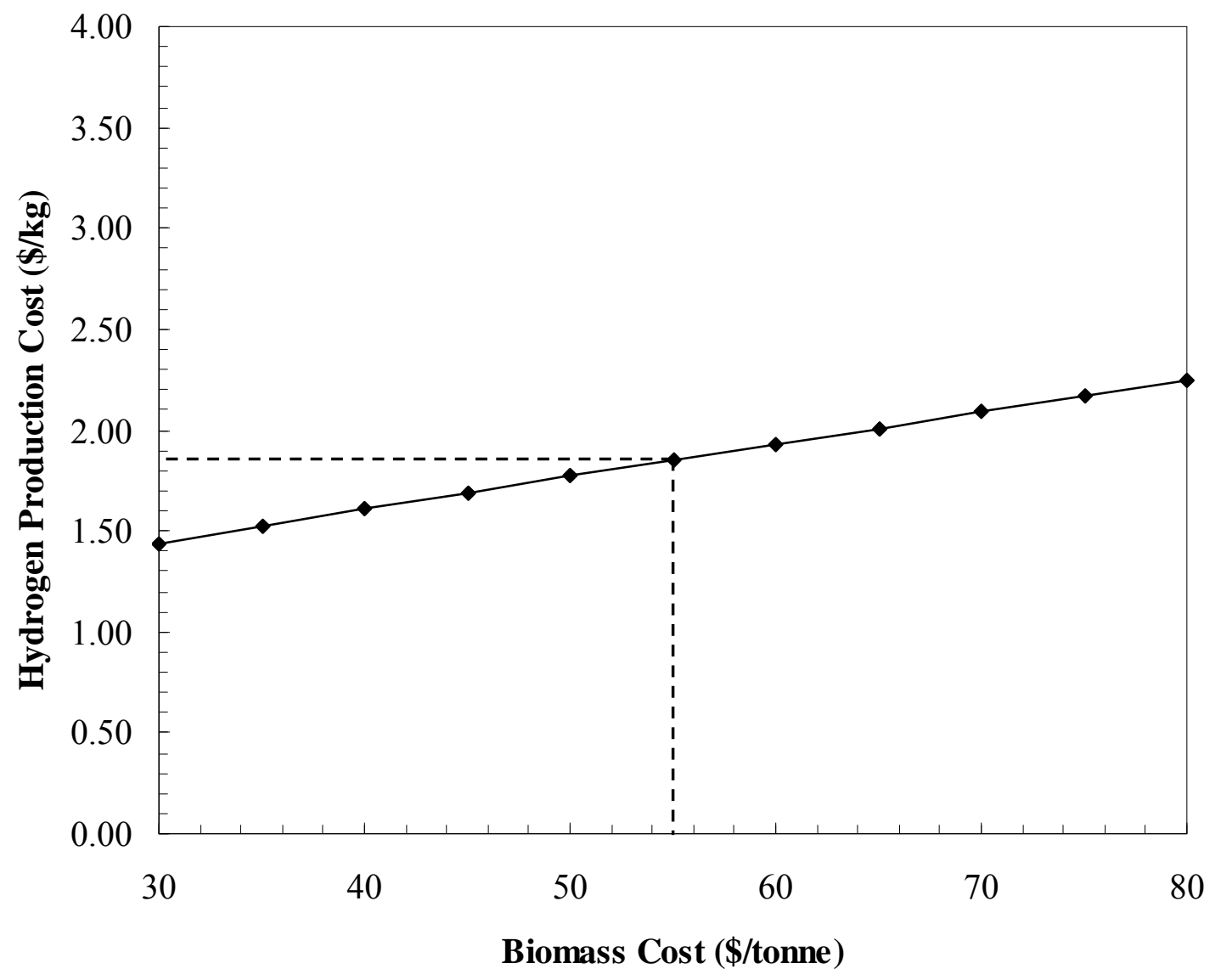

Figure 81 Effects of biomass cost on the hydrogen production cost 


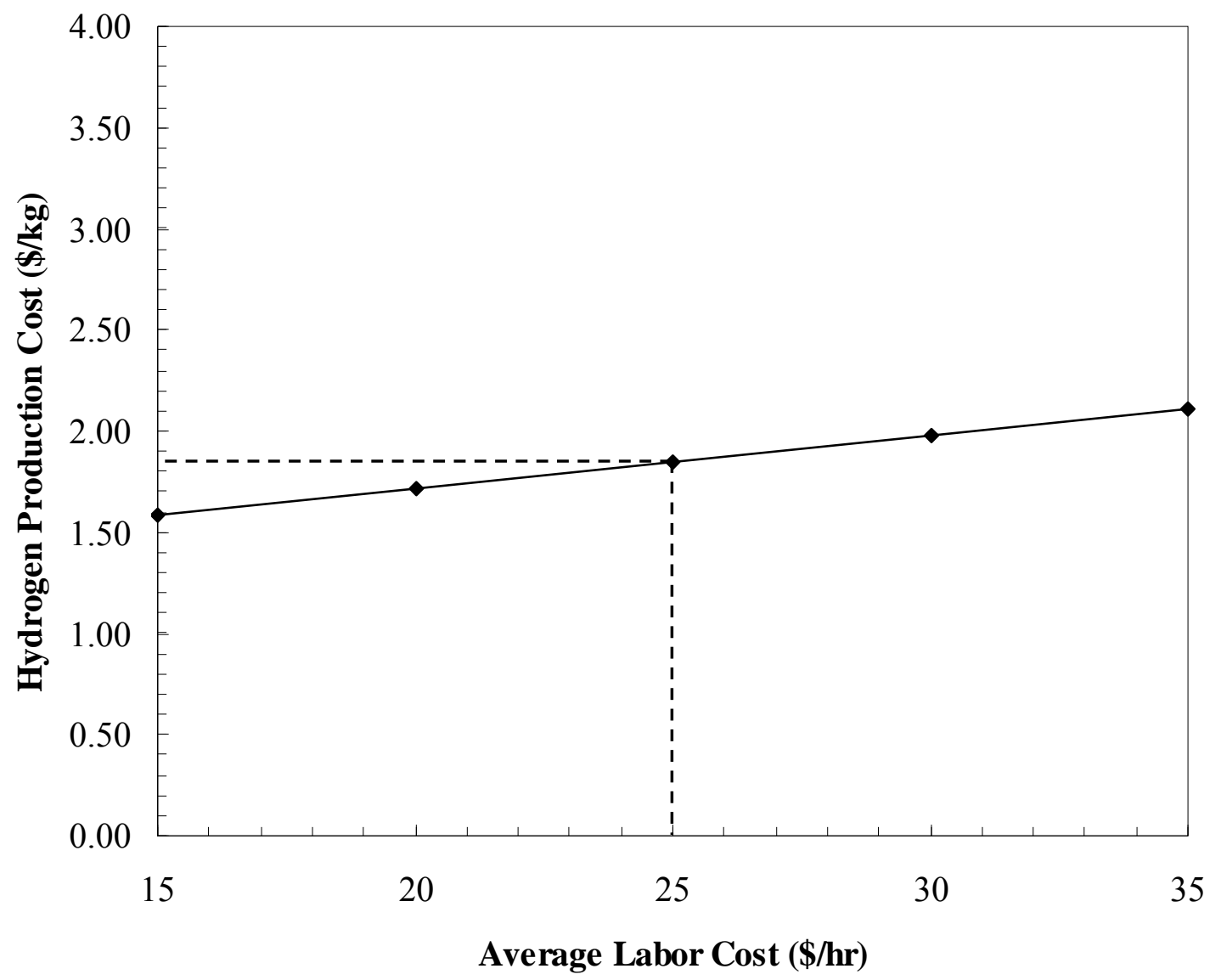

Figure 82 Effects of the labor cost on the hydrogen production cost 


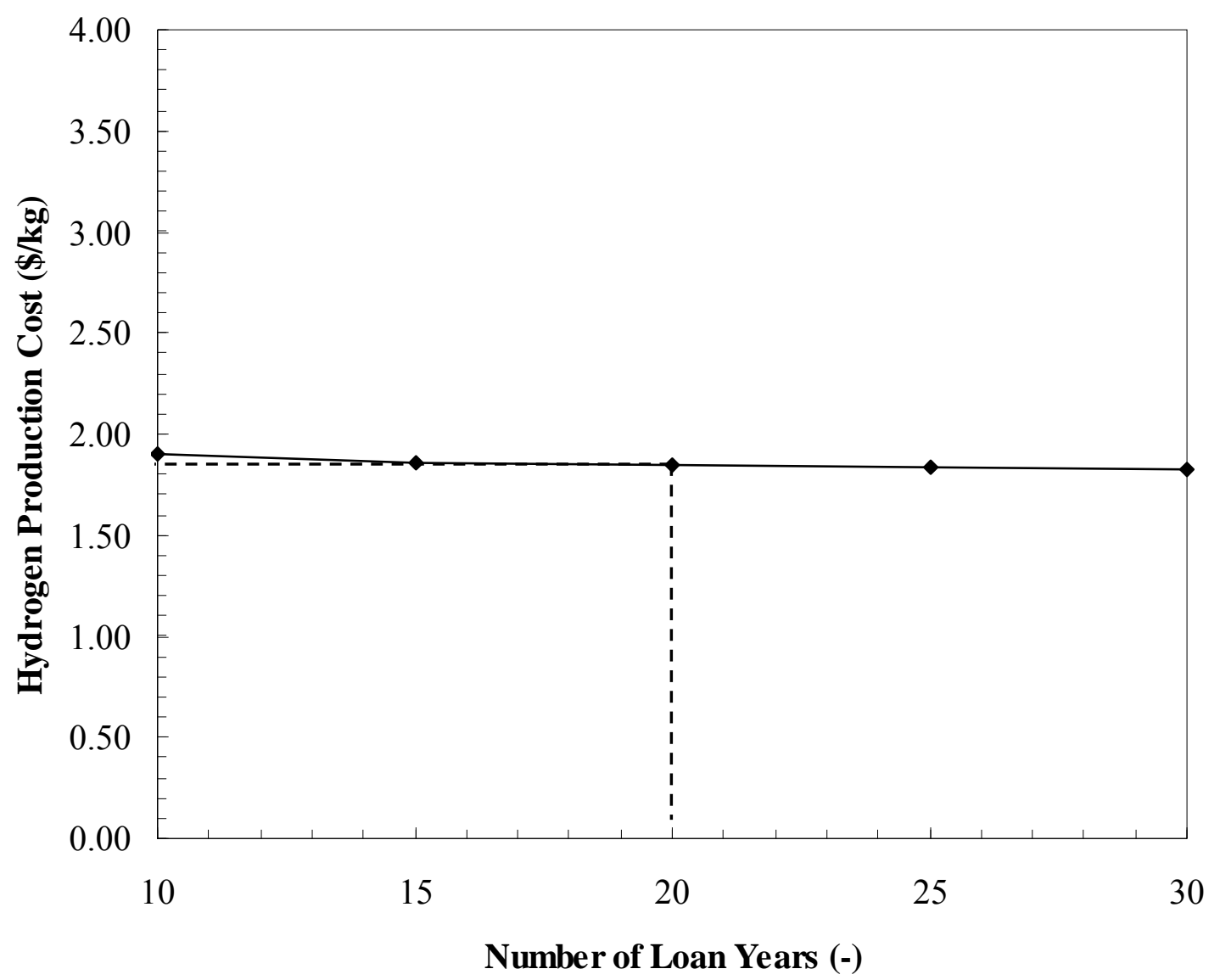

Figure 83 Effects of the number of loan years on the hydrogen production cost 


\section{Conclusions}

\section{Ballasted Gasifier}

The ballasted gasifier operates in a cycle, with a combustion phase followed by a pyrolysis phase. A larger amount of biomass can be processed through the reactor during the pyrolysis phase than during the combustion phase because essentially only very fast devolatilization of biomass occurs during the pyrolysis phase. Typically, the fuel flow rate during combustion was $55 \mathrm{~kg} / \mathrm{hr}$ compared to $180 \mathrm{~kg} / \mathrm{hr}$. These fuel flow rates produced a heating rate during combustion of about $19^{\circ} \mathrm{C} / \mathrm{min}$ and a cooling rate during pyrolysis of $36^{\circ} \mathrm{C} / \mathrm{min}$. The average durations of the combustion and pyrolysis phases were 13.0 and 7.0 minutes, respectively. Thus, $11.9 \mathrm{~kg}$ of biomass is required to heat the reactor to pyrolyze $21 \mathrm{~kg}$ of biomass.

From an energy perspective, it is advantageous to process very high flow rates of biomass through the reactor during pyrolysis as possible since it is completing with the fixed steam flow rate through the reactor for heat stored in the ballast and sand bed. However, this results in a mismatch between the combustion (heating) period and the pyrolysis (cooling) phases, which averaged 13.0 minutes and 7.0 minutes, respectively. Thus, producer gas is only being generated during $35 \%$ of the cycle. Simultaneous operation of three reactors would be required to give continuous production of producer gas. Alternatively, increasing the heating rate during the combustion phase could yield a better match between the times to complete the two phases of the reactor. This could be achieved by reducing heat loss from the reactor (see Section 5 for an analysis of this phenomenon) and reducing the amount of purge nitrogen admitted to the reactor via the fuel feed system.

The performance of ballasted gasifier was evaluated both in terms of the hydrogen content of the raw producer gas and the hydrogen content after steam reforming and water-gas shift reaction. Raw producer gas from the ballasted gasifier contained 18 vol-\% hydrogen compared to 8 vol- $\%$ hydrogen from the gasifier operated in air-blown mode. These numbers understate the potential of the ballasted gasifier to enhance hydrogen concentration because a substantial amount of nitrogen used to purge the fuel system entered the gasifier and diluted the producer gas during ballasted gasifier operation. Attempts to use smaller amounts of purge nitrogen were stymied by a tendency for the fuel feeder lines to overheat, which prevented proper feeding of fuel. However, if nitrogen purge could be substantially reduced through the use of an alternative fuel feed system, then hydrogen concentrations as high as 24 vol- $\%$ in the raw producer gas could be expected.

The raw producer gas contains substantial quantities of tar and carbon monoxide, which can be steam reformed and water-gas shifted, respectively, to additional hydrogen. When these systems were operated in conjunction with the ballasted gasifier, 54.5 vol- $\%$ hydrogen was obtained with most of the balance being $\mathrm{CO}_{2}$ and nitrogen from the fuel feeder purge gas. If the purge gas could be substantially eliminated, hydrogen concentration would reach 61 vol- $\%$, which closely approaches the theoretical maximum of 66 vol- $\%$. By comparison, a conventional, air-blown gasifier produced hydrogen at concentrations less than 30 vol.- $\%$ on a dry, inert-free basis, less than half that of the ballasted gasifier. 


\section{Moving Bed Granular Filter}

Both hot gas and cold gas evaluations of the moving bed concept for particulate filtration were evaluated. The hot gas trials were performed with a pilot-scale filter integrated with the biomass gasifier. Problems with maintaining gas seals in the sampling lines at high temperatures and confirming the existence of isokinetic sampling conditions limited conclusions that could be drawn from the hot gas trials. Analysis of pressure drop data showed evidence of the development of a dust cake, as hypothesized for this kind of filter. In some trials the filtration efficiency reached $97 \%$ although these results were not repeatable with any degree of reliability. For this reason, the major effort on this part of the project shifted to cold flow trials, which produced more reliable data.

The cold flow trials were performed in a laboratory-scale moving bed granular filter and suspended fly ash or char in an ambient temperature air stream for the tests. These trials found efficiencies for removing fly ash to be as high as $99 \%$. A simple particle removal model was able to predict the performance of the filter. The bimodally distributed coal fly ash tested in this study required separate layer efficiencies to be employed in the model for the two sizes of particles. Very fine particles and very coarse particles were the most efficiently removed by the filter while particles in the size range of $33-128 \mu \mathrm{m}$ appeared to limit overall filter efficiency to about $99 \%$ even for gas residence times in the filter on the order of $1 \mathrm{~s}$. However, these middlesized particles should be able to be removed by a high efficiency cyclone upstream of the moving bed filter, which would remove fine particles with efficiencies well in excess of $99 \%$. These experiments also showed that in excess of $86 \%$ of the fly ash was removed in the interfacial region where dusty gas first contacts the downward flowing granules of the moving bed.

In tests with other particulate matter (limestone, cornstarch, and oak char) it was demonstrated that filtration efficiency of the moving bed granular filter decreased with decreasing particle density and increasing irregularity of particle shape. Although oak char, consisting mostly of carbon, is expected to have very high van der Waals forces acting, the low density and highly irregular shape of this material resulted in collection efficiencies as low as $66 \%$. Since particle stickiness plays and important role in capture efficiency, it is hypothesized that higher efficiencies for char capture can be obtained by operating the filter at elevated temperatures, as would typically be employed in hot gas filtration. However, we were not able to perform such tests because the particle sampling methods were not suitable for elevated temperatures.

\section{Steam Reforming of Tar}

A tar conversion system consisting of a guard bed and catalytic reactor was designed for the purpose of improving the quality of producer gas from an air-blown, fluidized bed biomass gasifier. All three metal catalysts (ICI 46-1, Z409, and RZ409) proved effective in eliminating heavy tars ( $>99 \%$ destruction efficiency) and in increasing hydrogen concentration by $6-11$ vol$\%$ (dry basis). Space velocity had little effect on gas composition while increasing temperature boosted hydrogen yield and reduced light hydrocarbons ( $\mathrm{CH} 4$ and $\mathrm{C} 2 \mathrm{H} 4)$, thus suggesting tar destruction is controlled by chemical kinetics.

Although reactivity of the tar conversion system did not diminish during the 12-18 hours of testing, measurement of surface area and pore size distribution indicated the conversion of small 
pores into larger pores during high temperature operation. If this transformation were to continue, catalytic activity would eventually degrade. Furthermore, coke accumulated on both the dolomite and metallic catalysts although this might have been mitigated if higher steam/TOC ratios had been employed from the beginning of the tests.

Significant breakthrough of hydrogen sulfide from the guard bed occurred. However, relatively little sulfur accumulated on the Z409 and RZ409 catalysts, which were subjected to steam injection for the last $6 \mathrm{~h}$ of testing. This observation suggests that steam injection can regenerate metallic catalysts that have been poisoned by sulfur.

\section{Water-Gas Shift Reactors}

Two types of water gas shift reactor systems were evaluated. The first was a conventional twostage system consisting of a high temperature shift reactor followed by a low temperature shift reactor. This system, operated in conjunction of a steam reformer operated upstream of the shift reactors, was able to upgrade the hydrogen content of raw producer gas from the gasifier operated in air-blown mode from 8.6 vol-\% to 26.7 vol-\%. Carbon monoxide concentration of 14.3 vol- $\%$ in the raw producer gas was reduced to less than 0.2 vol-\%. Carbon monoxide conversion in the high temperature shift reactor reached $83 \%$ while the overall conversion in the two-stage shift reaction system reached $98.7 \%$. Although the steam reformer destroyed essentially all condensable tars and significantly reduced $\mathrm{C}_{2} \mathrm{H}_{4}$, it reformed very little of the $\mathrm{CH}_{4}$, which may reflect thermodynamic limitations as a result of inadequate temperature and too high of partial pressure of $\mathrm{CO}_{2}$.

Characterization of the catalysts by x-ray photoelectron spectroscopy showed that coke and small quantities of sulfur and chlorine deposited on the catalysts. BET analysis revealed loses in micropores and mesopores. Although no sign of catalytic deactivation was evident during the tests, these changes indicate the need for improvements in the design of the guard bed.

The second system evaluated was the combined catalyst-sorbent pellets employed in a single reactor. Since the tests performed were exploratory in nature, they were performed on simulated producer gas rather than producer gas generated from the biomass gasifier. These tests revealed that in the absence of $\mathrm{CO}_{2}$ adsorption, the hydrogen concentration never exceeded 70 vol- $\%$ due to the high concentration of $\mathrm{CO}_{2}$ arising from the water-gas shift reaction. However, when $\mathrm{CO}_{2}$ absorption by the pellets was allowed to occur, hydrogen concentrations exceeded 90 vol- $\%$. Furthermore, it appears that the catalyst was not only effective in promoting the water-gas shift reaction but in reforming some of the methane in the producer gas.

Following the test with steam and toluene some carbon was found deposited on the surface of the pellets and on the wall of the reactor above the bed indicating thermal decomposition of the toluene. This problem may be solved by increasing the steam to carbon ratio or lowering the operating temperature.

These results are highly encouraging because they show that it should be possible to upgrade producer gas in a single step to a product which is nearly pure hydrogen by employing a bed of material that combines a catalyst and sorbent in a single pellet. Further development of the material is required to provide a material which is highly stable and can be regenerated repeatedly and will last a long time. This will require optimizing the composition and preparation conditions for the material. 


\section{Measurements of Ammonia and Sulfur}

Detection of trace contaminants using continuous, on-line instruments is challenging because of the presence of a wide variety of organic and inorganic compounds that potentially can interfere with the desired measurements. Potentially attractive techniques like Fourier Transform Infrared Spectroscopy (FTIR), ion chromatography, and various continuous emission monitoring (CEM) instruments offered commercially proved inadequate for use with tar-laden producer gas. We eventually settled upon Drager tubes for repeatable although low resolution measurement of $\mathrm{H}_{2} \mathrm{~S}$ wet chemical methods for accurate though tedious measurement of ammonia.

In some tests, the concentration of $\mathrm{NH}_{3}$ appeared to substantially decrease with time. This unexpected behavior is believed to be related to the accumulation of high ash loading in the thimble filter used upstream of the impingers to remove particulate matter. We suspect that the $\mathrm{NH}_{3}$ was interacting with the ash, a caution for others using similar methods for ammonia detection.

\section{Sorbent Testing}

Two metal-based sorbents were tested for their ability to remove $\mathrm{H}_{2} \mathrm{~S}$ from biomass-derived producer gas. The $\mathrm{ZnO}$ sorbent, tested at $450^{\circ} \mathrm{C}$, was effective in reducing $\mathrm{H}_{2} \mathrm{~S}$ from $200 \mathrm{ppm}$ to less than $2 \mathrm{ppm}\left(>99 \%\right.$ reduction). The MnO-based sorbent, tested at $375^{\circ} \mathrm{C}$, showed evidence that it could reduce $\mathrm{H}_{2} \mathrm{~S}$ from $200 \mathrm{ppm}$ to less than $20 \mathrm{ppm}$ ( $>90 \%$ reduction), but anomalous behavior in the course of the experiment suggested that channeling or some related behavior was occurring through the packed bed, preventing more definitive conclusions from being drawn from the test.

The Wheeler equation, originally developed for analyzing the performance of gas masks, was employed in analyzing some of the data. This equation offers a means for determining both sorption rates and capacities during tests that are much shorter in duration than required to get actual saturation of a filter. This could be a very powerful methodology for the development of sorbents for the gasification industry. However, this methodology requires the ability to collect time series data on contaminant concentrations exiting the sorbent bed. The Drager tubes proved inadequate for this purpose. Recent installation of a GC-SCD system for semi-continuous measurement of sulfur species is expected to make this kind of analysis possible in future testing.

\section{$\underline{\text { Tar Measurements }}$}

Both the evaporative method of the IEA Protocol for tar measurement and a technique developed at Iowa State University were evaluated for their usefulness in measuring tar concentrations in biomass producer gas. Aging trials on tar/DCM samples collected by the IEA protocol demonstrated that storage of these samples, for as little as six hours, will produce errors in the reported tar concentration. The apparent tar concentrations can increase or decrease with time, depending upon the storage protocol. Thus, analysis of tar/DCM samples is recommended immediately upon collection of a sample.

The alternative tar analysis method developed at ISU is designed to simplify the analytical method while yielding results that are comparable to the evaporative method of the IEA Protocol (that is, determination of the heavy tar content of producer gas). This method, premised on condensing heavy tar at temperatures slightly higher than the boiling point of water, consists of 
simple gravimetric methods applied to a disposable tube. This "hot condenser" method yielded highly repeatable results that were within $10 \%$ of the values obtained by the IEA Protocol (for which no independent determination of accuracy is available).

\section{Ballasted Gasifier Model}

The purpose of this model was to accurately predict the thermal performance of the ballast system used in the biomass gasifier. A Receding Interface (RI) model was developed to account for the thermal barrier that develops within the ballast tubes as the LiF phase change material solidifies around the periphery of the tubes. This model predicts a continuous decrease in temperature during steam cooling of the fluidized bed, which is consistent with the behavior seen in experimental tests. The model demonstrates that the build-up of solid LiF during phase change slows heat transfer sufficiently to prevent a period of constant temperature reaction during the pyrolysis phase as was expected from earlier thermal models of the gasifier. The RI model predicts temperature profiles that are at slightly higher temperatures than are experimentally observed, but this is not unexpected since the model is one-dimensional.

Sensitivity analyses were performed on three parameters yielding the following conclusions:

- The time required to cool the bed to a particular temperature is proportional to the amount of phase change material employed in the ballast.

- Thermal conductivity of the lithium fluoride affects the cooling curves, but only during the period of phase change. Once the phase change material solidifies, the cooling curves are essentially identical.

- For a given mass of phase change material, smaller diameter tubes more closely approach the isothermal cooling curves of the idealized phase change process than do larger tubes.

\section{Estimated Cost of Hydrogen}

This evaluation compared the cost of hydrogen from biomass using the proposed ballasted gasifier and a conventional air-blown gasifier. For a plant producing 50 tpd of hydrogen, the total capital cost for the ballasted gasifier system is $\$ 31.99$ million as compared to $\$ 19.46$ million for an air-blown system. The higher capital costs associated with the ballasted gasifier system are a result of extra ballasted and steam generation equipment and lower hydrogen production levels as a result of lower biomass feeding rates.

Higher capital costs and lower ballasted gasification hydrogen production levels result in a hydrogen production of $\$ 2.43 / \mathrm{kg}$ compared to $\$ 1.85 / \mathrm{kg}$ for air blown gasification. These projections are within the range projected by other biomass-to-hydrogen analyses (between $\$ 1.38$ and $\$ 6.10 / \mathrm{kg}$ ) and within the 2010 target price of the U.S. DOE $(\$ 2.90 / \mathrm{kg})$. However, reductions in the cost of capital equipment or biomass feedstock will be required to meet the 2015 target price of $\$ 2.00 / \mathrm{kg} \mathrm{H}_{2}$. 


\section{References}

${ }^{1}$ Reed, T. editor. 1981. Biomass Gasification and Principles. Park Ridge, NJ: Noyes Data Corp.

${ }^{2}$ Battelle Columbus Laboratory. Process scale-up data summary. Report prepared for United States Department of Energy Richland Operations Office, November 27, 1985.

${ }^{3}$ Dallas I, McLellan, R, Scanlon, K, Smith, D, The gasification of wood using the oxygen donor process. In: Beenackers C, Van Swaay W, editors. Advanced gasification. Boston: Reidel Publishing, 1986;115-72.

${ }^{4}$ Mansour M, Durai-Swamy K, Voelker G. MTCI/ThermoChem steam reforming process for biomass. Proceedings of the Second Biomass Conference of the Americas, 1995.

${ }^{5}$ Pletka, R., Brown, R. C., and Smeenk, J., "Indirectly heated biomass gasification using a latent heat ballast. Part 1: Experimental evaluations," Biomass and Bioenergy 20, 297 - 305, 2001.

6 Brown, R. C., Smeenk, J., and Wistrom, C., "Design of a moving bed granular filter for biomass gasification," Proceedings of the Progress in Thermochemical Biomass Conversion Conference, Tyrol, Austria, September 17-22, 2000.

7 Milne, T. A., N. Abatzoglou, and R. J. Evans. Biomass Gasifier 'Tars': Their Nature, Formation, and Conversion. NREL/TP-570-25357, 1998.

8 Twigg, M.V. Catalyst Handbook; Second Edition; Wolfe Publishing Ltd., 1996; pp. 284-290.

${ }^{9}$ Pletka, R., Brown, R. C., and Smeenk, J. 2001. "Indirectly heated biomass gasification using a latent heat ballast. Part 1: Experimental evaluations," Biomass and Bioenergy 20, 297-305.

${ }^{10}$ Brown, R.C. 1993. Air Filtration An Integrated Approach to the Theory and Applications of Fibrous Filters. Pergamon Press.

${ }^{11}$ Amundson, N.R. 1948. A note on the mathematics of adsorption in beds, Journal of Physical Chemistry. 52:1153.

${ }^{12}$ Brown, R. C., Smeenk, J., and Wistrom, C. 2000. Design of a moving bed granular filter for biomass gasification, Proceedings of the Progress in Thermochemical Biomass Conversion Conference, Tyrol, Austria, September 17-22.

${ }^{13}$ Brown, R. C., H. Shi, Colver, G., and Soo, S. C. 2003. Similitude study of a moving bed granular filter, Powder Technology, 138(2-3), 201-210.

${ }^{14}$ Zhang, R. Q., Ma, W. Z., Preparation and method of high temperature shift catalyst for low steam to gas ratio, China National Patent: No. ZL 96102477.1, April 2000.

${ }^{15}$ Norton, G.A.; Brown, R.C. Wet chemical method for determining levels of ammonia in syngas from a biomass gasifier, Energy \& Fuels, accepted for publication, 2004. 
${ }^{16}$ Method 5 - Determination of particulate matter emissions from stationary sources, U.S. Environmental Protection Agency, http://www.epa.gov/ttn/emc/promgate/m-05.pdf.

${ }^{17}$ Pekka Simell and Pekka Stahlberg etc., Provisional Protocol for the Sampling and Analysis of Tar and Particulates in the Gas from Large-scale Biomass Gasifiers (Version 1998), Biomass and Bioenergy, Vol18, 2000: 19-38.

${ }^{18}$ Milne, T. A., N. Abatzoglou, and R. J. Evans. Biomass Gasifier 'Tars': Their Nature, Formation, and Conversion. NREL/TP-570-25357, 1998.

${ }^{19}$ Smeenk, J., Sadaka, S., Zhang, R., Brue, C., and Brown, R. C., Use of a thermally ballasted gasifier for the production of hydrogen from biomass, Proceedings of the Power Gen Renewable Energy Conference, Las Vegas, Nevada, March 1-3, 2004.

${ }^{20}$ Brown, R. C., Smeenk, J., Sadaka, S., Norton, G., Xu, M., Brown, N., and Brue, C., Thermochemical production of hydrogen from biomass, Proceedings of the Second World Conference and Technology Exhibition on Biomass for Energy, Industry, and Climate Protection, Rome, Italy, May 10 - 14, 2004.

${ }^{21}$ Ritzert, J., Brown, R. C., and Smeenk, J., "Filtration efficiency of a moving bed granular filter," Science in Thermal and Chemical Biomass Conversion Conference, Victoria, B.C., Canada, Aug. 30 - Sept. 2, 2004.

${ }^{22}$ Brown, R.C. 1993. Air Filtration An Integrated Approach to the Theory and Applications of Fibrous Filters. Pergamon Press.

${ }^{23}$ Thambimuthu, K.V. et al. 1978. Aerosol filtration in fixed granular beds. In Deposition and Filtration of Particles from Gases and Liquids. 107-120. The Society of Chemical Industry.

${ }^{24}$ Soo, Saw-Choon. 2002. Empirical model optimization of a moving bed granular filter for particulate removal. M.S. Thesis. Iowa State University.

${ }^{25}$ Shi, Huawei. 2002. Similitude modeling and experiments on a moving bed granular filter. M.S. Thesis. Iowa State University.

${ }^{26}$ Lippert, T. E. et al. 1981. Testing and verification of granular bed filters for removal of particulates and alkalies: High Temperature, High Pressure Particulate and Alkali Control in Coal Combustion Process Streams. Proceedings U.S. DOE Contractors' Meeting. Conf810249. 471-489.

${ }^{27}$ Shimada, Y. et al. 2002. Development of an apparatus for measuring adhesive force between fine particles. KONA. 20:223-229.

${ }^{28}$ Densities of common materials. Online: $<\underline{\mathrm{http}}$ //admin.engr.wisc.edu/quest/OPS/ops98.htm $>$, March 16, 2004. 
29 Zhang, R., Brown, R., Suby, A., and Cummer, K., "Catalytic destruction of tar in biomassderived producer gas,” Energy Conversion and Management (2004) 45 (7-8), 995-1014.

30 Zhang, R., Brown, R. C., and Suby, A., "Thermochemical generation of hydrogen from switchgrass," Energy and Fuels (2004) 18, 251-256.

31 Zhang, R., Cummer, K., Suby, A., and Brown, R. C., Biomass-derived hydrogen from an airblown gasifier," Fuel Processing Technology, to be published in early 2005.

${ }^{32}$ Milne, T. A., Evans, R. J., and Abatzoglou, N., Biomass Gasifier "Tars": Their Nature, Formation, and Conversion T.A. Milne and R.J. Evans, National Renewable Energy Laboratory Report NREL/TP-570-25357, November 1998.

${ }^{33}$ Pletka R, Brown RC, Smeenk J. 2001. Indirectly heated biomass gasification using a latent heat ballast. Part 2: modeling. Biomass and Bioenergy 20:307-315.

${ }^{34}$ Cummer, K. and Brown, R. C., Indirectly heated biomass gasification using a latent heat ballast. Part 3: Refinement of the heat transfer model, Biomass and Bioenergy, accepted for publication, 2004.

35 Incropera F, DeWitt D. 1996. Fundamentals of heat and mass transfer, $4^{\text {th }}$ ed. New York: Wiley.

${ }^{36}$ Pletka R. Latent heat ballasted biomass gasification. Ames, IA: Iowa State University, 1998.

${ }^{37}$ Oatley JA. Development of a latent-heat-ballasted biomass gasifier. Ames, IA: Iowa State University, 1994.

${ }^{38}$ Varlomov GB, et al. 1989. Thermophysical properties of fluoride crystals and melts. High Temperatures-High Pressures 21:647-656.

${ }^{39}$ SRI Chemical and Health Business Services per Chemical Engineering Magazine, The Utility of Hydrogen, September, 2001.

${ }^{40}$ Brooksbank, W., 2003. HydrogMax: Bridge to a hydrogen economy, Alchemix Corporation, $1 / 29 / 2003$

${ }^{41}$ Czernik, S, French, R., Feik, C. and Chornet, 2001. Production of hydrogen from biomassderived liquids. Proceedings of the 2001 DOE Hydrogen Program Review, NREL/CP-57030535, National BioEnergy Center, National Renewable Energy Laboratory, Golden, Colorado, USA.

42 Brown, R. C., 2003. Biorenewable Resources: Engineering New Products from Agriculture. Iowa State Press. Blackwell Publishing Company. 
${ }^{43}$ Bowen, D. 2003. A Techno-Economic Assessment of Hydrogen Production by Gasification of Biomass. Poster presented at the 2003 DOE Hydrogen and Fuel Cells Merit, Berkeley, California, May 19-22, 2003.

${ }^{44}$ Ghosh, D., Sagar, A., Kishor, V. Scaling up biomass gasifier use: an application-specific report. Energy Policy. Jan 13, 2005.

${ }^{45}$ Delvin, P. DOE Hydrogen Program EERE. Hydrogen Production and Delivery. Office of Hydrogen, Fuel Cell, \& Infrastructure Technologies. U.S. Department of Energy. May 24, 2004

${ }^{46}$ Spath, P.L., M.K. Mann and W.A. Amos (2003). Update of Hydrogen from Biomass Determination of the Delivered Cost of Hydrogen. Milestone Completion Report. National Renewable Energy Laboratory, Golden, Colorado.

${ }^{47}$ Spath, P., Aden, A., Eggeman, T., Ringer, M., Wallace, B., and Jechura, J. Biomass to Hydrogen Production Detailed Design and Economics Utilizing the Battelle Columbus Laboratory Indirectly-Heated Gasifier. NREL Technical Report, May 2005.

${ }^{48}$ Simbeck, D. and E. Chang. 2002. Hydrogen Supply: Cost Estimate for Hydrogen Pathways Scoping Analysis. National Renewable Energy Laboratory, Golden, Colorado. 